\title{
Abstracts from the Second Astrobiology Science Conference
}

\author{
NASA Ames Research Center, Moffett Field, California, USA \\ 7-11 April 2002
}

\section{Keynote presentations}

\begin{abstract}
Digital organisms for experimental evolution
C. Adam • Digital Life Laboratory; Jet Propulsion Laboratory; California Institute of Technology

Experiments in evolution are difficult for all the obvious reasons: the process is slow (such that macroevolutionary events occur only on a time scale of thousands to millions of years), and controlled conditions are hard to establish. As a consequence, evolution has, in the past, been mostly an observational and theoretical science, with little quantitative support. This has changed over the last decade, as more and more evolution experiments using microorganisms with short generational time have been conducted and their evolutionary dynamics studied in controlled conditions.

Digital organisms are a new form of life that is adapted to life in the computer. ${ }^{1,2}$ Because of its simplicity, it is ideal for experimental evolution, even though (and perhaps because) digital life does not share any ancestry with biochemical life on Earth. Digital organisms are selfreplicating computer programs that thrive in the memory of specially prepared computers. They adapt to a virtual world, and grow in complexity. Their main use is as an experimental organism, to test and study evolutionary theories and hypotheses. While several experiments have been conducted that mirror classic experiments performed with microorganisms, recently new evolutionary dynamics have been observed $^{3}$ in digitals that await confirmation with "biochemicals." References

1 Adami, C. (1998). Introduction to Artificial Life (Springer Verlag, NY).

${ }^{2}$ Harvey, I. (1999). Creatures from another world. Nature 400, 618-619.

${ }^{3}$ Wilke, C.O., Wang, J.L., Ofria, C., Lenski, R.E. \& Adami, C. (2001).

Evolution of digital organisms at high mutation rate leads to survival of the flattest. Nature, 412, 331-333.
\end{abstract}

\section{From interstellar polycyclic aromatic hydrocarbons} and ice to astrobiology

\section{Louis Allamandola $\bullet N A S A$ Ames Research Center}

The first part of this talk will describe how infrared studies of interstellar space, combined with laboratory simulations, have revealed the composition of interstellar ices (the building blocks of comets) and the nature of interstellar polycyclic aromatic hydrocarbons (PAHs).

The remainder of the presentation will focus on the photochemical evolution of these materials and astrobiology. Within a dense molecular cloud, the birthplace of stars and planets, the materials frozen into the ices are energetically processed by ultraviolet light and cosmic rays, producing complex organic molecules. Since these interstellar materials are thought to be the building blocks of comets and related to the organics in meteorites and micrometeorites, they are likely to have been important sources of complex materials on the early Earth. Thus, their composition may have played a role in the origin of life.
Life beneath glacial ice - Earth! Mars? Europa?

Carlton C. Allen $\bullet$ NASA Johnson Space Center, Stephen. E. Grasby • Geological Survey of Canada, Teresa G. Longazo $\bullet$ Hernandez

Engineering, John T. Lisle $\bullet$ United States Geological Survey, Benoit Beauchamp $\bullet$ Geological Survey of Canada

We are investigating a set of cold springs that deposit sulfur and carbonate minerals on the surface of a Canadian arctic glacier. The spring waters and mineral deposits contain microorganisms, as well as clear evidence that biological processes mediate subglacial chemistry, mineralogy, and isotope fractionation. The formation of native sulphur and associated deposits are related to bacterially mediated reduction and oxidation of sulphur below the glacier. A non-volcanic, topographydriven geothermal system, harboring a microbiological community, operates in an extremely cold environment and discharges through solid ice.

Microbial life can thus exist in isolated geothermal refuges despite long-term subfreezing surface conditions. Earth history includes several periods of essentially total glaciation. Ice in the near subsurface of Mars may have discharged liquid water in the recent past. Cracks in the ice crust of Europa have apparently allowed the release of water to the surface. Chemolithotrophic bacteria, such as those in the Canadian springs, could have survived beneath the ice of "Snowball Earth," and life forms with similar characteristics might exist beneath the ice of Mars or Europa. Discharges of water from such refuges may have brought to the surface living microbes, as well as long-lasting chemical, mineralogical, and isotopic indications of subsurface life.

\section{A culture-independent survey of the bacterial community in a radon hot spring in the Flinders Ranges of South Australia}

Roberto Anitori $\bullet$ Macquarie University, Australia, Cherida Trott $\bullet$ University of Auckland, David J. Saul $\bullet$ University of Auckland, Peter L. Bergquist $\bullet$ University of Auckland Medical School, New Zealand, Malcolm Walter $\bullet$ Macquarie University, Australia

Studies of the microbial biota inhabiting active and ancient terrestrial hot springs is relevant to the analysis of similar Martian sites. For instance, such research permits the development and refinement of appropriate methodologies (e.g. lipid biomarker analysis) for detecting evidence of present or past life on Mars. Paralana hot spring is located in the Mt Painter province of South Australia's Flinders Ranges. This region is highly mineralised as the result of ancient and current (Paralana) hydrothermal activity, with uranium and radium minerals predominating. Consequently, radon gas is released into the Paralana hot spring $(\sim 0.1-0.8 \mu \mathrm{Ci} / \mathrm{L})$ [Grant, 1938. Trans. Roy. Soc. S.A. 62, 357-365]. We have performed culture-independent 16S rRNA analyses (using bacteria-specific primers) of the microbial assemblages in nine Paralana samples. Water temperatures ranged from 48 to $63{ }^{\circ} \mathrm{C}$, and $\mathrm{pH}$ was neutral. Database searches and phylogenetic analyses conducted using Paralana 16S sequences indicated the presence of a diverse microbial community. Representatives of at least eight bacterial divisions were identified, including members of the cyanobacteria, green sulfur bacteria, nitrospira, the proteobacteria, and candidate division 
OP8. Interestingly, with the exception of one $16 \mathrm{~S}$ sequence, there was no evidence of previously described radiation-resistant microorganisms.

\section{Student research in astrobiology}

Jodi Asbel-Clarke $\bullet$ TERC, Jeff Lockwood $\bullet$ TERC, Dan Barstow $\bullet$ TERC

Building on experience in several successful scientist-student research partnership programs, the Center for Earth and Space Science Education (CESSE) at TERC is developing authentic research experiences for high school students and teachers in astrobiology. To enrich student learning in their early high-school integrated science astrobiology curriculum (to be published 2002-2003 by It's About Time), CESSE has created Habitable Worlds, an interactive online research tool for students. Habitable Worlds provides a student-friendly user interfacecalled an Exploration Portal to access image data from Pathfinder and other probes to enable students to pursue their own investigations about Earth, Mars, and other worlds.

As the next step, CESSE is beginning development on studentscientist partnerships in astrobiology following models used by other TERC projects. CESSE will develop tools and user interfaces for databases so that large numbers of students and teachers can engage in authentic research, while minimizing the time needed from scientists. With initial input from scientists and teachers, CESSE will develop protocols and instructions (with a background curriculum) so that students will be able to process data on a large scale, and then publish their results for the scientific community.

\section{Behavioral and physiological components of labor in pregnant rats}

L.A. Baer $\bullet$ NASA Ames Research Center; Lockheed Martin Space Operations, C.E. Wade $\bullet$ NASA Ames Research Center, A.E. Ronca $\bullet$ NASA Ames Research Center

Life beyond the home planet implies that civilizations will ultimately be established elsewhere in the universe. Successful reproduction is the hallmark of a species' adaptation to its environment and must be realized to sustain extra-terrestrial populations. Before taking this giant step, we need to understand the effects of gravity on critical phases of mammalian reproduction, viz., those events surrounding pregnancy, birth and the development of offspring. Our focus is on the role of gravity in the transition from prenatal to postnatal life. We previously observed altered frequencies of labor contractions in pregnant rats exposed to either spaceflight or centrifugation. During birth, rats exhibit characteristic behavioral expressions of labor. Lordosis contractions, consisting of an elongation of the dams body, are observed beginning several hours prior to neonate births, whereas vertical contractions, repeated rapid lifts of the abdomen, occur immediately preceding the birth of each neonate. In the present study, we analyzed changes in intrauterine pressure (IUP) using a telemetric blood pressure sensor that we modified for use in freely-moving rats. This technique provides a means for correlating behavioral expressions of labor contractions with IUP. A small telemetric blood pressure sensor was fitted within a fluidfilled balloon, similar in size to a full term rat fetus. On Gestational day 19 of the rats' 22-day pregnancy, a unit was surgically implanted within the uterus. The dams were simultaneously videotaped, enabling us to directly correlate IUP signals with behavioral expressions of labor contractions. Earlier phases of labor, consisting predominantly of lordosis contractions were characterized by lower pressures relative to later phases during which higher pressures and vertical contractions were frequently observed. These findings will be used to analyze gravitational influences on rat labor and birth. Supported by NIMH Grant MH46485, NASA Grants 121-10-40 and 121-10-50.

\section{The origin of habitats: observational constraints on star and planet formation}

John Bally • Center for Astrophysics and Space Astronomy; Center for Astrobiology; University of Colorado, Boulder

The majority $(\sim 90 \%)$ of stars in the sky form from giant molecular clouds in the vicinity of luminous, highly destructive, but short-lived massive stars. In these environments, proto-planetary disks are exposed to the dynamical effects of dense proto-clusters or multiple star systems, harsh radiation fields, and powerful winds. As a result, disks may be short lived. Indeed, in one of the nearest regions of star formation, the Orion Nebula, the Hubble Space Telescope has provided direct evidence for rapid disk destruction. The observed constraints imply that either planets form very rapidly, or that planetary systems will be relatively rare. I will review our current understanding of star and planet formation with an emphasis on observational results and on the processes that may affect the prospects for the eventual evolution of life.

\section{Mars soil formation and properties: the "acid fog" hypothesis in view of recent evidence}

Amos Banin $\bullet$ Hebrew University; NRC; NASA Ames Research Center We have proposed and tested experimentally a model that attributes the peculiar nature of Mars soil to it being a relatively "young" weathering product. The model suggests that Mars soil components formed at the microscopic rock-atmosphere interface during recent periods by a process involving chemical neutralization of acidic volcanic aerosols containing soluble $\mathrm{S}$ and $\mathrm{Cl}$ species. This "Acid Fog" formation scenario seeks to reconcile conflicting evidence for the presence of very soluble, water-transported sulfate and chloride salt minerals ("evaporites") in the soil with the lack of crystalline secondary iron oxides, clays, and carbonates that usually form during weathering in aqueous environments. The emerging new view of Mars based on the recent MGS imagery that show water seepage, surface ice-dust interactions, and volcanic activity on Mars as recent as the last 10 million years, adds strength to this model. A major conclusion from the "Acid Fog" hypothesis is that the lack of organic matter in Mars soil may be due to low cumulative meteoritic input rather than to exceptionally high oxidizing power. If true, this conclusion has profound effects on conceptual issues related to exobiology and the search for life on Mars as well as on practical programmatic questions related to robotic Mars exploration, Mars sample return and, eventually, to human exploration of Mars.

\section{Ultrasonic/sonic drill/corer (USDC) as a sampler and sensor platform}

Y. Bar-Cohen $\bullet$ Jet Propulsion Laboratory; California Institute of Technology, X. Bao $\bullet$ Jet Propulsion Laboratory; California Institute of Technology, N. Bridges $\bullet$ Jet Propulsion Laboratory; California Institute of Technology, Z. Chang • Jet Propulsion Laboratory; California Institute of Technology, B. Dolgin $\bullet$ Jet Propulsion Laboratory; California Institute of Technology, C. McKay $\bullet$ Jet Propulsion Laboratory; California Institute of Technology, S. Sherrit • Jet Propulsion Laboratory; California Institute of Technology, T. Peterson $\bullet$ Cybersonics, Inc.

The search for life in the Universe is one of the most important aspects of NASA's mission. Studies of life and fossilization processes in extreme environments show surface damage and drilling is essential for astrobiology studies. However, conventional techniques are limited by the need for high axial force. A novel USDC mechanism was developed by the authors to overcome this and other limitations of conventional drilling techniques. It has been shown to drill ice and a variety of rocks including granite, diorite, basalt and limestone and a scaled up version was demonstrated to allow for deeper drilling. A NASA ASTID task is underway to develop a breadboard of an ultrasonic percussion sampler and sensor platform for acquisition and characterization of the extracted core and powdered cuttings. The breadboard is designed to probe the medium prior to coring in order to optimize the selection of sites with a likelihood to contain biological signatures. A sample set will be chosen to represent biological markers and will be used to determine the degree to which the cored rocks and acquired powder cuttings are modified by the sampling process. The developed miniature, low-power, low-cost breadboard will be sought for technology insertion in future missions. 
Shear heating and solid-state convection on Europa: implications for astrobiology

Amy Barr $\bullet$ University of Colorado, Boulder, Robert Pappalardo $\bullet$ University of Colorado, Boulder, Francis Nimmo • University College, London, Eric Gaidos $\bullet$ University of Hawaii

Sustaining a biosphere in Europa's sub-surface ocean, if powered by radiation-driven chemistry, requires that oxidants generated on the surface of Europa be transported to the sub-surface ocean and fresh ice be replenished at the surface on a time scale of $10^{7}$ years [Chyba, Nature, 2000]. Traditional basal-heating models of solid-state convection can move material from the base of the ice shell to within $2-6 \mathrm{~km}$ of the surface on the geologically short time scale of approximately $10^{5}$ years. The formation of a stagnant lid where convective motion ceases near the surface of Europa inhibits the exchange of nutrient-bearing ice between the convective zone and the surface. We present a model by which material can be transported from the surface of Europa to the base of the ice shell on geologically short time scales through the coupled processes of draining of melt generated during the formation of ridges by shear heating along faults, and solid-state convection. We continue to investigate possible methods for diapers to pierce through the convective stagnant lid and extrude onto the surface.

\section{The effects of low sulfate concentrations on modern microbial mat communities: a long-term manipulation}

Brad Bebout $\bullet$ NASA Ames Research Center, Steve Carpenter $\bullet$ NASA Ames Research Center, David Des Marais $\bullet$ NASA Ames Research Center, Mykell Discipulo $\bullet$ NASA Ames Research Center, Mary Hogan $\bullet$ NASA Ames Research Center, Kendra Turk $\bullet$ NASA Ames Research Center

Microbial mats were widespread during the first $c a .2$ Gyr. of our biosphere's history. To better understand microbial ecosystems and their biomarkers under the low sulfate levels present in early oceans, we attempted a long-term ( $c a .1$ year) manipulation of sulfate in modern mats. Mats collected from salt ponds at Guerrero Negro, Baja California Sur were incubated in a Greenhouse "Collaboratory" at Ames. Mats were maintained in artificial seawater brine containing either: (1) sulfate levels normal for these mats $(70 \mathrm{mM})$, or (2) brine in which sulfate was replaced by chloride. Sulfate concentrations in the "low sulfate" brine gradually approached their lowest (to date) value of $0.1 \mathrm{mM}$ as sulfate was consumed and/or diffused out of the mat over a period of $c a .4$ months. During that period of time, a number of differences between the treatments emerged. Relative to the "low sulfate" mats, "normal sulfate" mats had: (1) increased penetration of $\mathrm{O}_{2}$ to the lower levels of the mat, (2) higher efficiencies of oxygenic photosynthesis, and (3) higher rates of nitrogen fixation. Rates of methane production by the mats increased greatly as sulfate concentrations fell below $c a .0 .2 \mathrm{mM}$. In contrast, "low" and "normal" sulfate mats had similar net rates of exchange of $\mathrm{O}_{2}$ and dissolved inorganic $\mathrm{C}$ between the mats and overlying water. Reduced sulfate levels have diverse impacts upon these ecosystems.

\section{Why astrobiology is important to biomedical research}

Steven A. Benner $\bullet$ University of Florida

Modern molecular biology is reductionist, an outgrowth of natural products chemistry from 19th century Germany, the chemistry of metabolic pathways, and the molecular analysis of DNA that began with the Watson-Crick structure in the 1950s. The reductionist approach produced the human genome project, which has provided no more (and no less) than the chemical structures of all of the molecules directly involved in human inheritance. The "structural genomics" database will soon follow, yielding a catalog of conformations of a sampling of the planetary proteome.

The power of the reductionist approach is evident throughout biology, and the major institutions that fund biomedical research have established it as their paradigm. This has often been, however, to the exclusion of planetary biology and historically-oriented research. Ac- cording to the Darwinian paradigm, however, functional behavior emerges only via historical processes (mutation and natural selection) that confer fitness upon a host organism surviving in a planetary ecosystem, only through global and historical analyses can function be assigned to the chemical structures that the reductionist approach has assembled. Lacking these analyses, the public has funded the sequencing of millions of nucleotides and thousands of crystal structures with little understanding of what they mean or how to use them in biomedical research.

Through its emphasis on the history of life, the NASA Astrobiology research program offers solutions to these problems. The history of life on Earth is chronicled in the geological strata and the fossil record, as well as the genomes of contemporary organisms. When examined together, these records provide detailed information about how proteins have evolved in tandem with the evolution of physiology and function, often in response to changes in the global ecosystem. Analysis of these combined records helps assign function to protein sequences, identify metabolic and regulatory pathways, suggest targets for diagnostics and therapeutics, and identify animal models to develop new drugs, among other goals of biomedical interest.

This talk will illustrate how this is done in human biology, with a focus on targets in reproductive endocrinology, inflammation, and metabolic disease. By showing how the past is the key to the present, these examples illustrate how planetary and historical analyses of genome and proteome databases work with reductionist science to provide an enhanced understanding how life works, how it interacts with the biosphere, and how it adapts to global change.

\section{Visualization of surfacial and internal structure and chemistry utilizing CT and neutron imaging \\ Rohit Bhartia $\bullet$ Jet Propulsion Laboratory, Alexandre Tsapin $\bullet$ Jet Propulsion Laboratory, Andrew Thompson $\bullet$ CMS, Robert Wang • CMS, Charles Suey $\bullet$ CMS}

Non-destructive techniques, such as X-ray and neutron tomography, have been used to provide an insight into the structure and chemistry of minerals and biological samples. With these methods, not only can details of surface topography and internal structures be resolved but atomic numbers of elements constituting structures of interest can also be derived. Also, a potential contamination of the interior of the sample can be estimated by visualizing fractures and/or pores. The newest scanning technology has a resolution of up to 4 micrometers/pixel. In order to fully understand the resulting data three-dimensional surficial and volumetric visualization techniques are being utilized. To do this we are using software created by CMS. Three-dimensional rendered images can be rotated, "sliced," and enhanced to display surfacial and hidden internal features for each technique. We present here data showing the potential possibility of determining the elemental composition of internal structures by superimposing images obtained by X-ray and neutron tomography. In addition, three-dimensional volumetric images will allow us to locate a region of interest as a future target for other more sensitive but usually destructive methods.

\section{Viruses and astrobiology}

Baruch S. Blumberg $\bullet$ NASA Astrobiology Institute; NASA Ames Research Center

Viruses are possibly the simplest form of life, so simple and so dependent on other organisms and cells that they are often considered to be outside the category of live organisms. However, early in viral evolutionary history they may not have been dependent on other cells for replication or have had a less dependent relation than in the modern world. Irrespective of this argument it is important to know more about viruses in the extreme conditions that may have existed on early Earth and on other planets. Astrobiologists are and will be studying viruses in the same locations at which they seek other extremophiles; in undersea vents, at geothermal sites, in the ice regions, deep under the earth, in acid and alkaline waters, and elsewhere. Many will be phages related to archae and bacteria, but some may have a more free-living style.

Viruses are remarkably versatile. Hepatitis B virus has only four reading frames, yet has developed a complex relation with its human 
host. It has adapted to transmission during periods that are vital to the life cycle of humans; from mothers to their children, from an infected sib to an uninfected family member, and sexually. It has developed mechanisms to negate the effects of the host immune system and allow the viral infected liver cell to remain intact for decades after infection, thereby increasing the possibility of transmission to another human host. It will be important to know whether any of the viruses living under extreme conditions, where humans would not thrive, can nevertheless be pathological. Viruses in extreme situations offer an exciting area for future study.

A recommendation has been made to establish a NAI Research Focus Group for Viruses. Members of NAI and others are welcome to sign up for this Group, if they have an interest in this area of research.

\section{Rapid ice giant planet formation and the implications for prebiotic chemistry}

Alan Boss $\bullet$ Carnegie Institution, George Wetherill $\bullet$ Carnegie

Institution, Nader Haghighipour $\bullet$ Carnegie Institution

The ice giant planets, Uranus and Neptune, represent a major unsolved problem in the theory of the origin of the Solar System. We propose here a radically new scenario for forming the ice giant planets. A gravitationally unstable protoplanetary disk forms giant gaseous protoplanets within about 1000 years, while dust grains coagulate and sediment to their centers, forming solid cores. The disk gas beyond Jupiter's orbit is removed in a million years by extreme ultraviolet (EUV) radiation from a nearby massive star, exposing the outermost protoplanets to EUV radiation, photoevaporating most of their gas in another million years, and leading to 15-Earth-mass cores with thin gas envelopes. This scenario implies that comets were subjected to a lengthy period of intense EUV irradiation, leading to photochemical production of surficial organic molecules.

\section{Exo/astrobiology in Europe: selected highlights} André Brack • Centre de Biophysique Moléculaire, CNRS, Orléans, France

The search for life beyond the Earth encompasses a large number of disciplines in order to satisfy the need for a reference, i.e. the origin and evolution of terrestrial life, and the necessity for exploring the solar system and the Universe. There are a number of centres in Europe active in the different fields covered by exo/astrobiology. A few, selected, recent results are given here.

Interstellar ice analogs made of $\mathrm{H}_{2} \mathrm{O}, \mathrm{CH}_{3} \mathrm{OH}, \mathrm{NH}_{3}, \mathrm{CO}$ and $\mathrm{CO}_{2}$ were ultraviolet-irradiated in a high vacuum at $12 \mathrm{~K}$ at the Leiden Observatory and analyzed in Orléans. Sixteen ${ }^{13} \mathrm{C}$-labelled amino acids, including six proteinaceous ones, were identified as racemic in the room temperature residue (Munoz Caro et al. 2002).

The fluid of the Rainbow hydrothermal field on the Mid-Atlantic Ridge contains $45 \%$ molecular hydrogen, a reactant that can reduce $\mathrm{CO}_{2}$ to generate hydrocarbons through Fischer-Tropsch-type synthesis. Linear saturated hydrocarbons with chain lengths between 16 and 29 carbon atoms were detected in the hydrothermal fluid (Holm and Charlou 2000).

Beagle-2, the exobiology lander of the ESA 2003 Mars Express mission, comprises an integrated suite of instruments to optimize the search for evidence of life on Mars in subsurface and rock interior samples. The package includes instruments to study sample mineralogy (composition, macroscopic and microscopic structural and textural features), organics (elemental, molecular), oxidation state and petrology (major and minor element composition). Environmental characteristics will also be measured (atmosphere, radiation budget, temperature, pressure, wind speed and direction, humidity, dust activity). The material will be sampled from protected sites (subsurface and rock interiors) with a mole and a small surface rock grinder and corer mounted on a robotic arm (Sims et al. 1999).

The "STONE" experiment, flown by ESA, was designed to test whether Martian sedimentary material could survive the terrestrial atmospheric. A basalt (inflight control), a dolomite (sedimentary rock) and artificial Martian regolith were embedded into the ablative heat shield of Foton 12, which was launched on September 1999. The collected entry samples have been analysed for their chemistry, mineralogy and isotopic compositions by a European consortium. Modifications due to atmospheric infall were tested by reference to the untreated samples. The dolomite sample was retrieved intact, although reduced to a depth of about $30 \%$ of its original thickness, suggesting that some Martian sediments could, in part, survive terrestrial atmospheric entry from space. Some kinetic isotopic fractionation accompanied the thermal degradation of the dolomite during re-entry, as evidenced by bulk isotopic measurements on different zones of the residual carbonate. The silica "fusion crust" from the associated sample holder exhibited a significant degree of isotopic exchange with atmospheric oxygen during re-entry (Brack et al. 2002).

References

Brack, A., Baglioni, P., Borruat, G., Brandstätter, F., Demets, R., Edwards, H.G.M., Genge, M., Kurat, G., Miller, M.F., Newton, E.M., Pillinger, C.T., Roten, C.-A. \& Wäsch, E. (2002). Do meteoroids of sedimentary origin survive terrestrial atmospheric entry? The ESA artificial meteorite experiment STONE, Planet. Space Science, in revision.

Holm, N. \& Charlou, J.L. (2000). Indications of abiotic formation of hydrocarbons in the Rainbow ultramafic hydrothermal system, Mid Atlantic Ridge. Earth Planet. Sci. Lett. 191, 1-8.

Munoz Caro, G.M., Meierhenrich, U.J., Schutte, W.A., Barbier, B., Arcones Segovia, A., Rosenbauer, H., Thiemann, W.H.-P., Brack, A. \& Greenberg, J.M. (2002). Photoproduction of amino acids in simulated interstellar pre-cometary conditions. Nature, in press.

Sims, M.R., Pillinger, C.T. \& 27 co-authors (1999). Beagle 2: a proposed exobiology lander for ESA's 2003 Mars Express Mission. Adv. Space Res. 23, No. 11, 1925-1928.

\section{Questioning the evidence for Earth's oldest fossils}

M.D. Brasier $\bullet$ University of Oxford, UK, O.R. Green $\bullet$ University of Oxford, UK, A. Steele $\bullet$ University of Oxford, UK, M. Van

Kranendonk $\bullet$ University of Oxford, UK, P. Jephcoat $\bullet$ University of Oxford, UK, K. Kleppe $\bullet$ University of Oxford, UK, J.F. Lindsay $\bullet$ University of Oxford, UK, N.V. Grassineau $\bullet$ University of Oxford, UK Morphological evidence for bacteria and cyanobacteria in the early Archaean has mainly depended upon 11 reported species of microfossil remains reported from the $3.46 \mathrm{Ga}$ old Apex cherts of the Warrawoona Group of Western Australia. ${ }^{1}$ However, the geological/biological context and morphology of this evolutionary benchmark have received little critical scrutiny. We have re-examined the type thin sections ${ }^{1}$ and compared them with samples re-collected from the same horizon. ${ }^{2} \mathrm{We}$ report on field mapping, light microscopy coupled to digital image analysis of the figured specimens, SEM (plus EDX), micro-Raman spectroscopy, carbon-, oxygen- and sulphurisotope measurements plus ToF-SIMS. We find that many of the filamentous structures are branched or formed in ways not shown in the original descriptions and illustrations ${ }^{1}$ because of the choice of focal depth and/or illustrated field of view. Our studies reveal chaotically branched pseudoseptate structures, some up to $36 \mu \mathrm{m}$ in diameter. ${ }^{2}$ We reinterpret all 11 holotypes in these samples as artefacts formed from amorphous graphite within multiple generations of metalliferous hydrothermal vein chert and volcanic glass. ${ }^{2}$ Although there is no support for primary biological morphology, a Fischer-Tropsch-type synthesis of carbon compounds and carbon isotopic fractionation is inferred for one of the oldest known hydrothermal systems on Earth.

References

${ }^{1}$ Schopf, J.W. (1993). Science 260, 640-646.

${ }^{2}$ Brasier et al. Nature (in press).

\section{Genetic diversity and dynamics of stromatolite- associated microbial populations}

Brendan Burns $\bullet$ The University of New South Wales, Australia, Brett Neilan $\bullet$ The University of New South Wales, Australia, David Relman - Stanford University, Don Lowe $\bullet$ Stanford University

The extant populations of cyanobacteria associated with present-day stromatolites are considered to be analogues of early life on Earth. To 
better understand stromatolite morphogenesis, we employed specific genetic probes to characterise the cyanobacteria responsible for stromatolite construction in a range of environments. Isolates of cyanobacteria (both unicellular and filamentous types) were collected from stromatolites in thermal springs, hypersaline lakes, and oceanic fringes on two continents. A PCR specific for cyanobacterial 16S rDNA was developed, and the resulting products of the DNA amplification reaction were sequenced and the data used to infer relatedness between the isolates studied and other members of the cyanobacterial radiation. A complete sequence was generated for the region from position 27 to 408 for 13 strains of cyanobacteria associated with stromatolites. All stromatolite-derived sequences were most closely related to cyanobacteria, as indicated by local sequence alignment. It was possible to correlate genetic identity with morphological nomenclatures, to expand the phylogeny of benthic cyanobacteria, and correlate results with geographic origin. Under the methods employed, only one cyanobacterial strain was detected in each sample, indicating the dominance of a specific clonal population of cyanobacteria at any one time in the stromatolite's biota.

\section{Biofilms: a mode of survival for bacteria in extreme environments \\ Anne K. Camper $\bullet$ Montana State University, Bozeman}

Bacteria have the ability to survive in extreme environments; they can be dried, frozen, chemically treated, and attacked by the human immune system and persist. This persistence is frequently enhanced when the organisms grow in communities on surfaces as biofilms. Examples of these robust attached communities are found in desert varnish, in acid and thermal aquatic environments, on deteriorating stone monuments, in the deep ocean in "smokers," in heavily disinfected or low nutrient water distribution systems, and on implants in the human body. For example, a clean water system may have very few suspended organisms, but a complex, well established biofilm may be present on the surfaces of the distribution system. This will occur even in the presence of a substantial disinfectant residual and very low organic carbon conditions.

Some of the earliest observations on the ability of biofilms to survive in extreme environments comes from the study of disinfectant and antibiotic resistance of biofilm bacteria. Doses/conditions that lead to the inactivation of individual suspended cells are typically inadequate for the reduction of biofilm bacteria. Even if some killing/removal is seen on the surface, the biofilms can and usually do quickly regrow. A considerable amount of research has been done to determine what factors lead to this decreased susceptibility, and the key concepts include diffusion/reaction of the antimicrobial agent with the biofilm, microenvironments within the biofilm that result in differences in metabolic activity and subsequent loss of susceptibility to the antimicrobial, and/or the presence of a distinct biofilm phenotype. Evidence for each of these concepts in environmental systems exists, and supporting data will be given. It is possible that these phenomena may contribute to the survival of bacteria in space stations and perhaps in other hostile, space environments.

Biofilm bacteria are also of key importance in human health. Specifically relating to space missions is the observation that biofilms will grow in the condensate on surfaces. Released biofilm particulates from these surfaces, as with those detached from cooling towers, ventilators for intensive care patients, and air conditioning systems are of importance in human health because of the inability of the immune system to clear these bacteria from the lung. A similar phenomenon is seen on medical implants; the organisms do not respond to antibiotic therapy, there is inflammation of the surrounding tissue, and substantial damage results. Dogma from experiments done in static systems has been that leukocytes in the immune system cannot penetrate these biofilms, and phagocytosis cannot occur. Recent experiments in flowing systems demonstrated that leukocytes can indeed penetrate the biofilm, but the ability to clear the organisms is still impaired. The implication is that there may be a change in the phenotype of these bacteria that allows them to elude the immune system.

\section{Europa as an abode of life}

Christopher Chyba $\bullet$ The SETI Institute

Life as we know it requires liquid water, a suite of biogenic elements, and a useful source of free energy. Here I review the prospects for life on Europa from the point of view of these three requirements. It is likely that Europa harbors an ocean of liquid water whose volume is twice that of Earth's ocean; I will review the evidence for this claim. Little is known about Europa's suite of biogenic elements, but even if Europa formed devoid of these materials, comet impacts over the age of the solar system place a lower bound on these inventories. Finally, there are a variety of possible sources of free energy available. The future search for life on Europa - receeding rapidly in the face of the cancellation of the Europa orbiter mission - should draw lessons from a thoughtful examination of Viking's search for life on Mars.

\section{Generalized and universalized definition of life}

Benton Clark $\bullet$ LMA and Center for Astrobiology, C.U.

Attributes cited for life exceed 100 . Here, fundamental characteristics are derived that are sufficiently generalized to encompass all that is "living" in the terrestrial biosphere. Other attributes are asserted to be derivative characteristics or innovative improvements, rather than essentials. The description is sufficiently universal for defining a variety of theoretical lifeforms (a) present on another planet, (b) created by testtube biotechnology, (c) artificial inorganic emergent life, or (d) candidates for the first primitive forms of life. Past problematical cases, e.g. viruses, prions, sterile organisms, organelles, anucleate cells, endospores, test-tube life, computer viruses, replicated robots, and evolutionary algorithms are unambiguously classifiable, but it is concluded that two terms are required: lifeform and organism, both "alive," with the former having proliferative properties enabled by embedded instructions, and the latter as physical entities capable of energy channeling activities guided by internal instructions. This provides a rational framework for the detection of lifeforms yet to be discovered. Life is found to be more fundamental than attributes such as evolution, molecularspecies, compartmentalization, homeostasis, hierarchicalcomplexity, enzyme catalysis, entropy, speciation, intelligence, independence, or spatial or temporal scale.

\section{Responses of $C$. elegans to hypergravity treatment}

C. Conley $\bullet$ NASA Ames Research Center, Udranszky, NASA Ames

Research Center

All organisms on Earth have evolved at unit gravity (1G), and thus are probably adapted to function optimally at $1 \mathrm{G}$. However, with the advent of space exploration, it has been shown that organisms are capable of surviving at much less than $1 \mathrm{G}$, as well as at greater than $1 \mathrm{G}$. Organisms subjected to increased $\mathrm{G}$ levels exhibit alterations in physiological processes that compensate for novel environmental stresses, such as increased weight and density-driven sedimentation. These physiological adaptations illustrate the plasticity of organisms when presented with environmental conditions in which they could not possibly have evolved. Investigating the mechanism(s) behind these adaptations may uncover biological pathways that have not been identified previously.

To establish whether C. elegans exhibit altered physiological processes as a result of several days exposure to moderate levels of increased $G$ (2-200G), we have developed a centrifugation protocol using axenic liquid cultures of $C$. elegans and the 1 -foot diameter centrifuge at NASA-ARC. After exposure to hyperG for 4 days, C. elegans cultures exhibited retarded growth relative to stationary control cultures. Immediately after being removed from the centrifuge, many worms were immobile and did not display a "tap-response" to brief mechanical stimulation. Mobility and the tap-response were regained by two hours after the return to unit gravity, and the behavioral responses and growth of cultures were subsequently indistinguishable from controls. Growth at $10 \mathrm{G}$ for 4 days produced alterations in steady-state mRNA levels, as evaluated by whole-genome microarray analysis on mRNA from centri- 
fuged C. elegans compared with parallel cultures of stationary controls. Fewer than 100 genes out of the entire genome were upregulated or downregulated to a statistically significant degree. Very few stressresponse genes were upregulated, indicating that the centrifuged cultures were under only mild stress. Most of the genes are of unknown function, but a number of them show similarity to proteins possibly involved in signal transduction, including G-protein receptor subunits and transcription factors.

These data demonstrate that $C$. elegans do respond to chronic increases in $\mathrm{G}$ level at both a behavioral and molecular level. Work is ongoing to establish how the genes that we have identified may be involved in the responses of $C$. elegans to increased gravity relative to other types of chronic mechanical stimulation.

\section{Astronomy village: investigating the solar system; astrobiology in middle school}

Steven Croft $\bullet$ Center for Educational Technologies

Science teaching is more effective when it involves hands-on activities and problem solving. "Astronomy Village: Investigating the Solar System" (AVISS) is a CD-ROM-based multimedia software package that effectively draws middle school students into the world of the astrobiologist. In AVISS, students enter a simulated environment and are presented with two important questions: Does life exist elsewhere in the solar system? What is the surface of Pluto like? The first question invites exploration of the conditions required for life. The second leads into the study of the processes that shape the planetary environments in which life develops. Both of these questions remain unanswered at present. (We have found that students are drawn to problems for which scientists don't already know the answers.) As students investigate these questions, they are presented with basic information, real data, and conflicting viewpoints. They are then are allowed to explore, analyze data, and present their own conclusions. The conclusions students arrive at are not as important as the evidence and logic they use to support their conclusions. In AVISS, the important thing is the journey - not the destination.

AVISS is an award-winning program that has been thoroughly tested in classrooms across the United States and has been found to be effective in engaging and teaching young students.

\section{Terrestrial extremophiles under Europan surface conditions}

J. Brad Dalton $\bullet$ NASA Ames Research Center, Hiromi Kagawa $\bullet$ NASA Ames Reseach Center; SETI Institute, Rakesh Mogul $\bullet$ NASA Ames Research Center; Educational Associates

Spectral measurements by the Galileo NIMS instrument suggest abundant hydrated sulfuric acid (Carlson et al. 1999) and magnesium sulfur salts (McCord et al. 1998) in disrupted, reddish-brown surface regions on the surface of Europa. Upper bounds on concentrations of these compounds (Dalton 2000) are consistent with extremophilic habitats on Earth (Rothschild \& Mancinelli 2001). Chaos regions (Spaun et al. 1999) appear linked with subsurface environments (Pappalardo \& Head 2000) which could contain sufficient energy and nutrients (Zolotov \& Shock 2000; Chyba \& Phillips 2001) for microbial organisms.

Samples of the archaea Sulfolobus shibatae, which lives in environments containing sulfuric acid and magnesium sulfate salts were obtained along with the highly radiation-resistant extremophile Deinococcus radiodurans and common Escherichia coli. Infrared spectra of these reddish and brown microbes were measured in a cryogenic vacuum chamber at temperatures of 200-80 K. Afterwards, small portions of each sample were still viable. While $D$. radiodurans had the highest survival rate, some of the $S$. shibatae and E. coli survived as well. Furthermore, the infrared spectra, compared with Galileo NIMS spectra, indicate that mixtures of sulfur compounds, and microbes which depend upon them, could currently exist in the near-surface Europa environment.
Past sea surface temperature derived from tree rings Hector D'Antoni • NASA Ames Research Center, Ante Mlinarevic • San Jose State University

The general distribution of the biota in South America is related to the properties and operation of the climate system. The Hadley Cell in the tropical region and the Ferrell Cell in mid latitudes control atmospheric circulation. Between both cells the jet stream is formed. The seasonally occurring vortex centered in the South Pole affects climate in the troposphere by introducing anomalies. Precipitation is related to the ocean-atmosphere interface and, in South America, predominantly dominated by the Atlantic Ocean. The "El Niño" Southern Oscillation (ENSO) affects these patterns in four regions of South America (Ecuador, Northern Amazonia, Eastern Brazil, and Eastern Argentina) with a large increase of precipitation (Ecuador, Argentina), drought (Northern Amazonia) and higher temperature (Ecuador, Eastern Brazil). These changes affect tree growth in these and other regions of South America. Using dendrochronology data from 25 sites in South America we trained an artificial intelligence algorithm. With the resulting model we reconstructed the Atlantic and Pacific oceans past sea surface temperature (SST) for the period 1246-1991. Atlantic SST appears more stable around $24.5^{\circ} \mathrm{C}$. Pacific past SST varies over a much larger range around $21^{\circ} \mathrm{C}$ and reflects ENSO episodes in the past that are longer than the instrumentally recorded ones.

\section{Rapid rates of change in South American vegetation linked to the "El Niño" southern oscillation}

Hector D'Antoni • NASA Ames Research Center, David L. Peterson • NASA Ames Research Center, Ante Mlinarevic • San Jose State

University

To study the astrobiology topic of rapid rates of change on Earth we selected South America with its sharp precipitation gradients $(10 \mathrm{~m}$ in the Pacific coast of Colombia to $0.18 \mathrm{~m}$ in Patagonia), and temperature gradients $\left(26^{\circ} \mathrm{C}\right.$ in Amazonia and $8{ }^{\circ} \mathrm{C}$ in Patagonia). During the course of the last 10000 years these conditions supported the continent's remarkable biodiversity. The "El Niño" Southern Oscillation (ENSO) is a short-term, intensive perturbation to the "normal" environmental conditions of the continent. We hypothesized that vegetation quickly responds to the environmental changes introduced by the ENSO and sensors mounted in satellites can monitor the responses. Our analysis of the normalized differences vegetation index (NDVI) from the Advanced Very High Resolution Radiometer (AVHRR) at 8-km resolution from 1982 through 2001 has validated our hypothesis. During this period, two significant environmental changes have occurred in the pampas of Argentina: (1) after the 1982-83 ENSO a lake was formed near Pehuajo in a region that has been under intensive agriculture for over 200 years; (2) the "dry pampa" has undergone a rise of the ground water table with the formation of numerous ponds even in the area of the "Calden" dry forest.

\section{From ET to $A B$ : public perceptions of astrobiology} and how museums can help

L. Danly $\bullet$ Denver Museum of Nature and Science; Denver University, C. Tang $\bullet$ California Academy of Science, Martin Weiss $\bullet$ New York Hall of Science

The general public holds perceptions of extra-terrestrial life that, fed by the media and popular myth, can be a far cry from the scientific discipline of astrobiology that we all know and love. Yet the scientific search for life beyond Earth is no less engaging or compelling a story than science fiction, and it can be brought to life in new ways in the informal settings of 21 st century museums, science centers, and planetaria. In addition to the inherent interest of the subject to the public, astrobiology provides an opportunity for lay audiences to understand the process of conducting scientific inquiry and the excitement of cutting-edge, multi-disciplinary research. Experiences and perspectives are shared by an astronomer, a geologist/paleontologist, and a biologist, each of whom is engaged in developing a multi-million 
dollar exhibition and program designed to introduce astrobiology to broad and diverse audiences in an informal museum setting.

\section{Why is the universe bio-friendly?}

Paul Davies $\bullet$ McQuarie University, Australia

The emergence of life in the universe depends critically on certain key properties of the laws of physics. Many scientists have noted that even slight changes in the form of the laws would make life (at least as we know it) impossible. So is the bio-friendliness of the universe just an incredibly fortunate accident, or is there a deeper explanation? An increasingly popular view is that what we call "the universe" is but a small component in a much larger "multiverse," consisting of regions - or even entire disconnected spacetimes - in which the laws take on a variety of forms. The propitious nature of the laws in our universe is then neatly explained as an anthropic selection effect. But how seriously can we take the existence of these other universes? And can such theories be tested experimentally?

\section{Self-assembly of organic molecules and the origin of cellular life \\ David W. Deamer $\bullet$ University of California, Santa Cruz}

Did life arise from pre-existing cellular structures, or did cellular life develop only at a later evolutionary stage? There were no large molecules like nucleic acids and proteins available on the prebiotic Earth to direct the assembly processes characteristic of life today, so the first membrane structures related to early cellular life must have appeared by selfassembly. All membranous boundary structures in contemporary forms of life incorporate a lipid bilayer as the primary barrier to free diffusion of solutes. Bilayer-forming molecules are amphiphilic, with a hydrophilic "head" and a hydrophobic "tail" on the same molecule. Were amphiphilic molecules present in the mixture of organic compounds available on the early Earth? One way to address this question is to assume that the mixture of organic compounds in carbonaceous meteorites resembles components available in extraterrestrial infall. A small fraction $(\sim 5 \%)$ of stony meteorites contain up to several percent of their mass in the form of organic carbon. An insoluble polymer composed largely of covalently linked polycyclic aromatic hydrocarbons is the most abundant organic material, and a series of organic acids represents the most abundant soluble fraction (Cronin et al. 1988).

Fifteen years ago, it was not known whether amphiphilic molecules were also present in carbonaceous meteorites. Samples of the Murchison meteorite were therefore extracted in an organic solvent commonly used to isolate membrane lipids from biological sources (Deamer \& Pashley, 1989). When this material was allowed to interact with aqueous phases, one class of compounds with acidic properties was clearly capable of forming membrane-bounded vesicles. In more recent work (Dworkin et al. 2001) organic compounds with similar properties were synthesized in precometary ices simulating those present in dense molecular clouds. The observation that membranes can self-assemble from such amphiphilic components makes it plausible that membrane-bounded structures were present at the time of life's origin.

The next step is to develop laboratory models of simple cellular systems with macromolecules encapsulated by phospholipid bilayer vesicles (Chakrabarti et al. 1994; Walde et al. 1994). In a recent study, Apel et al. (2002) demonstrated that mixtures of fatty acids and fatty alcohols having relatively short chain lengths ( $8-10$ carbons) can also form stable vesicles and encapsulate a functional enzyme. More complex systems incorporating both a catalytic polymerase and a DNA template have been prepared, and RNA synthesis in the vesicles has been observed (Monnard \& Deamer 2002). These results provide a useful perspective on the most primitive forms of cellular life. It seems likely that amphiphilic hydrocarbon derivatives were present on the early Earth that could self-assemble into bilayer boundary structures. These would have been sufficiently permeable to allow passage of ionic substrates required for polymerization of macromolecules such as RNA, yet maintain those macromolecules within a boundary. Furthermore, specific groupings of macromolecules would be maintained in one microenvironment, rather than diffusing away from one another. This would allow evolutionary selection of complex molecular groupings to occur, a process that could not take place as easily in mixtures of molecules free in solution.

References

Apel, C., Mautner, M. \& Deamer, D.W. (2002). Self-assembled vesicles of monocarboxylic acids and alcohols: conditions for stability and for the encapsulation of biopolymers. BBA Biomembranes 1559, 1-9.

Chakrabarti, A., Breaker, R.R., Joyce, G.F. \& Deamer, D.W. (1994). Production of RNA by a polymerase protein encapsulated within phospholipid vesicles. J. Mol. Evol. 39, 555-559.

Cronin, J.R., Pizzarello, S. \& Cruickshank, D.P. (1988). Organic matter in carbonaceous chondrites, planetary satellites, asteroids and comets. In Meteorites and the Early Solar System, eds Kerridge J.F. \& Matthews, M.S., pp. 819-857. University of Arizona Press, Tucson AZ.

Deamer, D.W. \& Pashley, R. (1989). Amphiphilic components of the Murchison carbonaceous chondrite: surface properties and membrane formation. Orig. Life Evol. Biosphere 19, 21-38.

Dworkin, J.P., Deamer, D.W., Sandford, S.A. \& Allamandola, L.J. (2001). Self-assembling amphiphilic molecules: synthesis in simulated interstellar/precometary ices. Proc. Natl. Acad. Sci. USA 98, 815-819.

Monnard, P.-A. \& Deamer, D.W. (2002). Models of primitive cellular life: polymerases and templates in liposomes. J. Mol. Evol. (submitted).

Walde, P., Goto, A., Monnard, P.-A., Wessicken, M. \& Luisi, P.L. (1994). Oparin's reactions revisited: Enzymatic synthesis of poly(adenylic acid) in micelles and self-reproducing vesicles. $J$. Am. Chem. Soc. 116, 7541-7547.

\section{Kerogen distribution in oxide-bearing microfossils from the gunflint formation}

\section{Brad De Gregorio $\bullet$ Arizona State University, Tom Sharp • Arizona} State University

Microfossils in ancient cherts provide the primary record of early life on Earth, yet little is known about the mineralogy and ultrastructure of these important biomarkers. Taphogenesis is known to alter organic remains by various processes, such as perminerallization, dissolution, and metamorphism. However, it is still unclear how these processes affect the internal microstructure of such remains, or even how to recognize bona fide preserved microfossils. Amorphous carbon may be a key criterion for identifying microfossils. Using TEM imaging and EDS spectrometry, we have found kerogen in red-banded, microfossiliferous cherts from the Mink Mountain locality of the Gunflint Formation. The microfossils, visible in thin section as "nets" of black, red, or brown filaments, are mostly composed of hematite grains as large as $1 \mu \mathrm{m}$, surrounded by smaller patches of kerogen. Normally, fine-grained hematite crystals would appear red in thin section, but the presence of carbon intermixed with the microcrysts can alter the color macroscopically, resulting in the varied filament coloration. Despite an oxidizing depositional environment, resulting in the formation of hematite crystals, carbon is still present in a measurable abundance. This suggests that small amounts of kerogen may be used as a biomarker for silicified filamentous features composed primarily of oxide minerals.

\section{Photosynthetic eukaryotes: from the acquisition of organelles to the colonization of the land}

\section{Charles F. Delwiche $\bullet$ University of Maryland}

The origin and evolution of land plants (i.e. embryophytes) represents one of the seminal events in the history of life on Earth. Not all plants are land plants: the land plants are a single group of organisms that include mosses, ferns, pine trees and flowering plants. They are derived from structurally simple but highly diverse organisms that are referred to collectively as "algae." Modern studies definitively show that the ancestors of land plants were freshwater green algae (Kenrick \& Crane 1997), but only recently have we been able to pinpoint the select groups that are the nearest living relatives of land plants. Molecular phylogenetic analyses show that the order Charales is the sister group to all land plants, with the Coleochaetales sister to the land plant/Charales lineage (Karol et al. 2001). Both of these orders are members of a larger 
group, the division Charophyta. The Charophyta show extensive morphological diversity, have fossil records that extend back well over 400 million years (Feist \& Feist 1997), and appear in molecular phylogenetic studies to constitute one of the two primary lineages of green algae, which are themselves a fundamental crown group of eukaryotes. When the land plant lineage took hold upon the continents, there were profound changes in the global environment including dramatic modification of the erosion regime, correlated changes in marine nutrient inputs, and striking fluctuations in atmospheric chemistry (Knoll 1994). These changes took place not only because of the ability of land plants to survive on land, but also because their structure and physiology permitted them to make efficient use of resources, anchor themselves effectively and in so doing stabilize the substrate and permit the formation of modern soils, and maintain metabolic activity for long periods of time even in the absence of rainfall and surface moisture. Many groups of green algae live in the terrestrial environment, but only one of these - the land plants - has radiated into a wide range of habitats. It is not known why the land plant lineage has been so successful. Traits that are likely to have played a role in their success include cell wall biochemistry, desiccation resistance and tolerance, structural complexity, and various reproductive strategies. In all probability the success of the land plant lineage was not the result of a single "key innovation," but an emergent property resulting from complex interactions among these and other features of the lineage. Comparative study of the properties of diverse aquatic and terrestrial algae can be used to identify properties that were important in the colonization of the land and how they interact. We have been able to document the relationships among many relevant groups of algae with molecular phylogenetic methods, and are currently engaged in work to characterize their genetic capabilities. It is our hope that understanding how the land plant lineage came to dominate the terrestrial environment will help determine whether a plant-like colonization of the land is an inevitable consequence of planetary and biotic evolution.

References

Feist, M. \& Feist, R. (1997). Oldest record of a bisexual plant. Nature $\mathbf{3 8 5}$, 401.

Karol, K.G., McCourt, R.M., Cimino, M.T. \& Delwiche, C.F. (2001). The closest living relatives of plants. Science 294, 2351-2353.

Kenrick, P. \& Crane, P.R. (1997). The Origin and Early Diversification of Land Plants: a Cladistic Study. Smithsonian Institution Press, Washington, DC.

Knoll, A.H. (1994). Neoproterozoic evolution and environmental change. Early Life on Earth, ed. Bengston, S., pp. 439-459. Columbia University Press, New York.

\section{Microbial mats help us understand our origins and search for distant biospheres}

David J. Des Marais • NASA Ames Research Center

The hierarchical organization of microbial ecosystems determines the rates of processes that shape Earth's environment, define the stage upon which major evolutionary events occurred, and create biosignatures in sediments and atmospheres. In cyanobacterial mats, oxygenic photosynthesis provides energy, organic substrates and oxygen to the ecosystem. Incident light changes with depth in the mat, both in intensity and spectral composition, and counteracting gradients of oxygen and sulfide shape the chemical microenvironment. A combination of benefits and hazards of light, oxygen and sulfide promotes the allocation of the various essential mat processes between light and dark periods and to various depths in the mat. Microbiota produce hydrogen, small organic acids, nitrogen and sulfur species. Such compounds fuel a flow of energy and electrons in these ecosystems and thus shape interactions between groups of microorganisms. Coordinated observations of population distribution, abundance, and activity for an entire community are making fundamental questions in ecology accessible. These questions address those factors that sustain the remarkable diversity of microorganisms that are now being revealed by molecular techniques. These questions also target the processes that shape the various kinds of biosignatures that we will seek, both in ancient rocks from Earth and Mars, and in atmospheres of distant planets beyond our Solar System.

\section{Diversity of dissimilatory sulphite reductase (DSR) genes in the Guaymas Basin}

Ashita Dhillon $\bullet$ Marine Biological Laboratory, Andreas P. Teske $\bullet$ Woods Hole Oceanographic Institution, Mitchell L. Sogin $\bullet$ Marine Biological Laboratory

Hydrothermal systems support a diverse microbial ecosystem and have even been linked to the origin of life on Earth and possibly other planets, e.g. Europa. Guaymas is a deep semiclosed basin in the Gulf of California and is a site of hydrothermal activity. Evolutionary studies of phylogenetically conserved genes may one day help us to interpret potential microbial life elsewhere in the universe. In this study we use marine sediments (Core B) from a site close to the hydrothermal activity of the Guaymas Basin. The 16S rRNA gene sequences were used to characterize bacterial communities in the core B sediments and to infer an evolutionary framework. We are particularly interested in the sulfitereducing bacteria, which are a diverse group of anaerobic microorganisms that use sulfate as a terminal electron acceptor. Methane could account for $52 \%$ of the electron donor requirement for the peak sulfate reduction rate detected in the sulfate-methane transition zones. The maximum anaerobic methane oxidation rates coincide with local maximum rates of sulfate reduction. Dissimilatory sulphite reductase (DSR) protein catalyses the reduction of sulfite to sulfide, an essential step in the anaerobic sulfate respiration pathway. To compliment diversity studies based upon rRNA genes, we characterized the $1.9 \mathrm{~kb}$ fragment of DSR genes from various divergent clones.

\section{What the tree of life really means}

$W$. Ford Doolittle $\bullet$ Canadian Institute for Advanced Research; Dalhousie University, Halifax, Nova Scotia, Canada

Thanks to the pioneering work of Carl Woese and the enthusiastic endorsement of the molecular evolutionary community, ribosomal RNA (in particular small subunit, or SSU rRNA) gene sequences have become the gold standard for phylogenetic reconstruction at many levels, defining the trunk, branches and twigs of the universal Tree of Life. To this molecule we owe the signal discovery of the tripartite (Bacteria/Archaea/Eukarya) structure of the living world and a new appreciation of the astonishing extent of microbial diversity. However, accepting this or any other single gene as a marker of universal organismal phylogeny requires accepting a model of vertical inheritance - the assumption that transfer across species lines has not been a significant evolutionary force. And, for many genes in Bacteria and Archaea, we now know this to be seriously untrue, in both the short evolutionary run and the long.

First I will discuss violations in the short run - the mounting evidence that even strains of the same bacterial species can differ by more than $20 \%$ in gene content. The notorious pathogenic E. coli O157:H7, for instance, has 13 hundred genes not found in the common laboratory strain K12 (which has more than 500 not found in O157). I will describe our recent data indicating that interstrain variation in gene content is just as great in Thermotoga maritima, a free-living bacterial hyperthermophile, so such variability is likely to be general and not just a consequence of the arms race between superbugs and us, their hosts. Furthermore, such variation must be in significant part the result of lateral gene transfer (LGT): logic demands this unless we are willing to entertain a model of ever-decreasing genome size, with inter-strain differences always reflecting differential losses of information from a gene-rich species ancestor.

But if strains of the same species, separated by only thousands or at most millions of years, can suffer what amounts to a turnover of $20 \%$ of their genes, what reason is there to believe that any genes have escaped LGT in 3.8 billion years? Assessments of the fraction of any single genome that looks "foreign" have been made in several independent ways, and usually come to no more than $30 \%$ but it can be 
shown that all methods have potential systematic errors that could result in a serious underestimation. Another way to look at this problem, then, is to ask whether there is a stable core of genes shared by all genomes that cannot be, or at least hasn't been transferred. Many have argued on theoretical grounds that essential genes whose products interact with many other genes' products should function too poorly in a new and foreign genomic environment to be transferred, and that genes for many components of the transcriptional and translational machinery are such. However, LGT of transcriptional and translational genes is now well known, and there is no compelling evidence for a stable core of genes that is: (1) traceable back to the "root" of the universal tree, and (2) comprises more than 5-10\% of any contemporary genome. Other models for the relationship between gene trees and genome and organismal evolution that can account for existing reliable comparative genomic data should be entertained. I will present two: the "shifting core" model, which is compatible with many whole-genome analyses, and the related "nested gene pool model." I will suggest that what we are really after in our attempts to reconstruct a universal organismal phylogeny is a history of all speciation events and that although there are many useful and interesting things to be done here there may never be a direct and simple mapping of this history to the phylogeny of genes.

\section{Cosmic origins: a traveling science exhibit and education program}

Paul Dusenbery $\bullet$ Space Science Institute, Cherilynn Morrow $\bullet$ Space Science Institute

The Space Science Institute (SSI) of Boulder, Colorado has recently been funded by the National Science Foundation to develop a 3000 square-foot traveling exhibit called Cosmic Origins. Exhibit visitors will explore the birth of stars, the formation of planets, and learn about the methods and tools scientists use to conduct the search for planets beyond our solar system and life beyond Earth. The exhibit content will address age-old questions that form the basis of NASA's Origins and Astrobiology programs: Where did we come from? Are we alone? The exhibit will begin its 3-year tour in mid 2004; ASTC will manage the itinerary. In addition to the exhibit, our project will include workshops for educators and docents at host sites, as well as a public website that will use a virtual rendering of exhibit content. Current partners in the Cosmic Origins project include NASA missions (e.g. SIM, SIRTF, and Kepler), the SETI Institute, TERC, the New York Hall of Science, the Lawrence Hall of Science, and the Denver Museum of Nature and Science.

\section{Mineral substrates as a source of metabolic energy for primary biomass production in seafloor hydrothermal environments}

Katrina Edwards • Woods Hole Oceanographic Institution, Thomas McCollom $\bullet$ Woods Hole Oceanographic Institution, Daniel Rogers $\bullet$ Woods Hole Oceanographic Institution

It appears likely that the earliest microbes to evolve on Earth, as well as any extant microbial communities on planetary bodies such as Mars or Europa, relied on chemical reactions rather than sunlight for metabolic energy and biomass production (i.e. chemolithoautotrophy). Minerals that are out of equilibrium with their environment represent a widespread source of chemical energy for microbes on Earth and elsewhere. We are studying microbes in hydrothermal environments that obtain metabolic energy from mineral substrates, particularly metal sulfides. Mineral samples retrieved from the vicinity of seafloor hydrothermal vents reveal dense colonies of microbes. Phylogenetic analyses of the microbial communities indicate one prominent group of species related to Thiomicrospira, a common sulfur-oxidizer. However, most of the population has no close relatives in culture. In an effort to characterize the physiologies of these organisms, we have used a variety of methods to culture mineral-oxidizing species, targeting Fe-oxidizers in particular. We have isolated $\sim 20$ novel strains, all of which are capable of growth on minerals as the only energy source (sulfides, siderite, basaltic glass), but are incapable of growth on simple organic compounds. Our results indicate that minerals are widely used as an energy source for primary biomass production in hydrothermal ecosystems.

\section{Analysis of changes in Arabidopsis thaliana statocyte structure in response to hypergravity}

Reza Ehsanian $\bullet$ San Jose State University; Science and Technology Corp., Stephanie D. Hopkins $\bullet$ NASA Ames Research Center, David K. Bruck $\bullet$ San Jose State University, Jeffrey D. Smith $\bullet$ NASA Ames Research Center

Statocytes, specialized gravity-sensing cells, serve the important function of providing higher plants with the directional cue needed for proper growth and development. Amyloplasts are redistributed within statocytes of the root cap due to changes in the gravitational environment leading to the hypothesis that they function as the statoliths (gravity sensors) for higher plants. The overall focus of this research in our laboratory was to determine the mechanisms by which plants adapt to changes in the gravitational environment. A combination of traditional electron microscopic analysis and three-dimensional cell reconstructions were used to test the hypothesis that amyloplast size is dependent on position, which is changed under altered gravity conditions. The 1 -ft diameter centrifuge facility at NASA Ames Research Center was used to expose seedlings of Arabidopsis thaliana to chronic hypergravity stimulation. Samples were processed for transmission electron microscopy and electron micrographs of longitudinal root tip images were scanned and quantitative morphometrics were performed using an NIH image. Three-dimensional reconstructions were made from electron micrographs utilizing the ROSS (reconstruction of serial sections) software program at the Ames BioVIS Technology Center. Reconstructed cells were analyzed to elucidate relative intracellular and intercellular positions of the amyloplasts. This method is less subjective and more accurate than traditional morphological techniques used to reconstruct and analyze ultrastructure. The ability to visualize and measure entire cells in a three dimensional environment helps to determine the effects of gravity on amyloplast position and its relationship to amyloplast size with much greater certainty than through traditional morphometric methods. The results of these analyses help us show how plastid position is related to size, and how increased gravity conditions change the size and starch content of amyloplasts. This leads us to a more accurate proposed mechanism by which higher plants adapt to changes in the gravitational environment. (This research was supported by NASA Ames Research Center.)

\section{A vanguard for astrobiology: a bio-rover for Mars} Alex Ellery $\bullet$ Kingston University, London, UK, David D. WynnWilliams • British Antarctic Survey, Cambridge, UK, Howell G.M. Edwards $\bullet$ University of Bradford, UK, David L. Dickensheets $\bullet$ Montana State University, Bozeman, MT, Chris Welch $\bullet$ Kingston University, London, UK, Andy Curly $\bullet$ Kingston University, London, $U K$

We present a proposed European mission architecture for experimental astrobiology on Mars. Its aim is to seek unequivocal biomolecules in situ within profiles of near-subsurface sedimentary strata along a transect in a potential former habitat. The mission represents a natural extension to the UK-led Beagle 2 mission currently under development for launch in 2003. Our proposal, called Vanguard, comprises the landed segment of the mission designed nominally for the Mars Express spacecraft bus. The entry, descent and landing is conceived to be similar to that adopted on Pathfinder and Beagle 2. The landed segment comprises of a small base-station lander to provide relay communications to the orbiter, a small robotic (Endurance) rover similar to Sojourner, and three moles (Orpheus) similar to that adopted on Beagle 2 , which are mounted onto the rover. The rover will carry a miniature laser-Raman spectrometer as its primary instrument connected by optical fibre-carrying tethers to the moles, which will each carry a sidescanning sensor head. The moles will be deployed at three well-separated surface sites selected during the mission and will penetrate to a nominal depth of $5 \mathrm{~m}$ below the Martian surface. 


\section{Comparative biology of replicators}

Andrew D. Ellington $\bullet$ University of Texas, Austin, Matthew Levy •

University of Texas, Austin

Autocatalytic replicators based on nucleic acids, peptides and organic molecules have been engineered previously. We will compare the salient properties of these replicators, and derive a nomenclature for understanding how they differ from one another and other possible replicators. We suggest that nucleic acid and peptide replicators are digital, composed of discrete units that can be combined into an informational code, while the organic replicators that have so far been examined are not. Nucleic acid replicators are discrete, in that they can alternate between stable states, whereas the peptide replicators that have so far been examined are more continuous, capable of giving rise to a variety of products. The structural complementarity of discrete replicators is of necessity greater than that of other replicators. However, increased complementarity leads to product inhibition. These facts imply a fundamental conundrum: the less discrete a replicator is, the less information it can transfer; the more discrete a replicator is, the more its evolution is limited by parabolic growth. Thus, it is proposed that nucleic acid or other replicators can best arise from autocatalytic cycles, rather than semi-conservative copying. An experimental example of such an autocatalytic cycle will be explored.

\section{Ecological stoichiometry of stromatolitic cyanobacteria-diatom mats and hydrobiid snail} grazers in thermal springs at Cuatrocienegas, Mexico James Elser $\bullet$ Arizona State University, Marcia Kyle • Arizona State University, John Schampel • Arizona State University, James Watts • Arizona State University

The emerging discipline of Astrobiology focuses on the study of the origins and early dynamics of life on Earth and beyond. We are studying modern analogs of early-Earth systems in Cuatrocienegas, Mexico. In particular, we are employing the perspective of ecological stoichiometry to assess the impacts of phosphorus supply on stromatolite-forming cyanobacterial-diatom mats and on their grazers, locally endemic hydrobiid snails. Algal mats at Cuatrocienegas have extremely high $\mathrm{C}$ : $\mathrm{P}$ and $\mathrm{N}: \mathrm{P}$ ratios, much higher than algal biomass in oceans and lakes and more similar to vascular plant biomass in terrestrial ecosystems. These high $\mathrm{C}: \mathrm{P}$ and $\mathrm{N}: \mathrm{P}$ ratios probably place stoichiometric food quality constraints on their consumers. In field experiments, $\mathrm{C}: \mathrm{P}$ and $\mathrm{N}: \mathrm{P}$ in mat biomass responded strongly to enrichment of inorganic nutrients, as algal mats actively removed nutrients (especially $\mathrm{P}$ ), resulting in lower $\mathrm{C}: \mathrm{P}$ and $\mathrm{N}: \mathrm{P}$ ratios after enrichment. Data on the $\mathrm{P}$ demands of snail grazers are also being collected, as well as on their growth response (via RNA:DNA measurements) to P-enrichment of the algal mats upon which they graze. Our data may have implications for factors constraining the success of early metazoa during times when stromatolitic algae were the dominant life forms on Earth.

\section{Constructing innovation: the intersection of genetics, ecology and environment \\ Douglas Erwin • Smithsonian Institution}

Successful evolutionary innovation requires ecological opportunity, genetic and developmental possibility and an appropriate environmental setting. Most models of innovation assume that ecological opportunities (niches) exist largely independent of the organisms that fill them. Similarly, genetic and developmental mutations have been viewed as proposing novel modifications that are then filtered by ecology and the physical environment. Missing from such perspectives is a recognition that organisms both actively construct their own environment and create environments for others. The advent of novel metabolic activities, the spread of oxygenic photosynthesis, trapping and binding of sediment by microbial mats and later by multicellular algae, the origination of plants and animals and their spread onto land each involved the construction of ecospace rather than the filling of a pre-existing or predefined ecospace. A similar process may occur through regulatory evolution. For example, the transition from metazoans built upon simple cell-specification to those with more complex morphogenetic networks constructed new developmental possibilities, and thus new ecological opportunities. Innovation, then includes a positive feedback process that sequentially expands the carrying capacity of a system subject to the limits imposed by available energy, species/abundance relationships and similar factors.

\section{Microbial processes and their coupling to ice dynamics in perennial ice covers on Earth: insights for Europan environments}

Christian H. Fritsen $\bullet$ Desert Research Institute, John Priscu $\bullet$ Montana State University, Ed Adams $\bullet$ Montana State University, Peter Doran $\bullet$ University of Illinois at Chicago

The conditions possibly supporting life within and below Europa's icy shell are presently under study, yet the physical and chemical conditions therein are equivocal. Bodies of water on Earth with perennial ice covers (e.g. oceans and lakes) provide insights into environmental conditions and processes that may exist on other planetary bodies and hence be useful in our evaluation of the possibility of life beyond our planet.

Perennial ice covers, up to $20 \mathrm{~m}$ thick in the McMudro Dry Valleys, contain cyanobacteri-adominated assemblages, whereas sea ice typically supports assemblages dominated by eukaryotic photoautotrophs. Despite ecosystem energy being derived from solar radiation, the cycling of materials within these habitats support heterotrophs and chemoautotrophs that can survive in an ice environment not supported by contemporaneous photoautotrophy. Material transport and cycling in perennial sea ice is often coupled to brine movement in the process of desalination and ice accretion on the surface and base of the ice cover. Material transport and cycling in freshwater ice covers is often linked to atmospheric deposition, ice movement imposed by sublimation and accretion processes and internal melting. Similar processes may occur on the Europan ice cover at different scales. It is the specifics of these similarities that will guide future life-detection missions.

\section{A conceptual model of a robotics laboratory to search for life in Martian water and permafrost} David Gan • University of California, Berkeley, Lawrence Kuznetz • NASA Johnson Space Center

When liquid water is found on Mars, it must be tested for potability by microbiological and chemical means to ensure the survival of the Mars Mission and future colonization. Eventually, these tests must be performed by manned missions with the risk of exposure to opportunistic pathogens. The advantage of robotics testing will provide early warning of such possible hazards and life forms on Mars even before the Sample Return Program. Lederberg, Vishniac et al. had proposed robotic laboratory designs in the 1960s, but they were not space-qualified. The Viking II's three biological experimental results were inconclusive and are still under debate. The Mars Orbiter Camera findings suggest that possible subsurface water may still be present on Mars and a robotics exploration program is planned to search for life forms within the next ten years. We need to utilize recent advances in microscopy, automated cultivation systems, biological stains, computers, computer imagery and molecular biology for robotics exploration. This proposed first stage will use the cultivation, stains and microscopy. Later, the second stage will employ molecular biology by searching for non-specific longchain DNA/RNA structures using micro-chips proposed by Deamer. The search for non-terrestrial life is one of our quests in the new century.

\section{NASA's Mars exploration program: the trajectory of knowledge, with first results from ODYSSEY}

\section{James B. Garvin $\bullet N A S A$ Headquarters}

The scientific exploration of Mars is amidst a revolution catalyzed by the continuing observations of the Mars Global Surveyor (MGS) orbiter, and now amplified by the first mapping results of the Mars Odyssey (MOd). These scientific reconnaissance missions have provided a new framework for addressing the most pressing questions about 
Mars, including those centering around "biological potential, now and in the past." The quest for understanding the biological potential of Mars, and how any records of biological activity can be isolated and found is perhaps the overarching driver in our scientific trajectory for exploring the Red Planet. The recommendations given to NASA by the National Academy of Sciences Committee for Planetary and Lunar Exploration (COMPLEX, draft report November 2001: Assessment of NASA's Mars Exploration Program) as well as by the Mars Exploration Program Assessment Group (MEPAG; report edited by Professor Ron Greeley, July 2001) both reaffirm the importance of assessing the biological potential and prospects for the planet Mars. Indeed, these critical assessments both stress the multi-disciplinary nature of the search for astrobiological "signals" on Mars, by recommending that a program of refined reconnaissance, followed by definitive in situ analyses at locations that offer the best preservation possibilities for biosignatures, and ultimately involving a campaign of robotic Mars Sample Return (MSR) missions. NASA has adopted a simple scientific strategy for exploring Mars during the present decade that has been described as "seek, in situ, sample," in which current missions (MGS, MOd) are the first cycle of "seeking," while upcoming landed missions (2003 Mars Exploration Rovers or MER, and 2009 Mars Smart Lander or MSL) constitute the second cycle of in situ exploration, with an intervening next-generation reconnaissance step (2005 Mars Reconnaissance Orbiter or MRO). Ultimately, at some point in the next decade, the first of a series of MSR missions will be undertaken, ideally targeted to the most promising localities for which samples that record potential biosignatures can be accessed and returned to Earth. Key to this entire strategy is the 2009 landed mission known as "Mars Smart Lander." NASA is looking to the astrobiological community to help direct this mobile scientific laboratory, which as been referred to as a "Mobile Geobiological Explorer," to carry with it a next generation of in situ life inference instruments that will help make profound progress in assessing the biological potential of the locally accessible planet Mars. The current decade of Mars exploration by NASA and international colleagues (ESA, CNES in France, ASI in Italy, CSA in Canada, and Russia) is unprecedented in the history of human scientific exploration, and integration of astrobiological drivers into this amazing "decade of discovery" is vital.

\section{Carbonyl sulfide as a greenhouse gas for early Mars Mimi Gerstell • California Institute of Technology, Yuk Yung • California Institute of Technology, Hari Nair $\bullet$ Jet Propulsion Laboratory}

We had previously proposed ${ }^{1}$ that 0.1 ppmv $\mathrm{SO}_{2}$ in a 2-bar $\mathrm{CO}_{2}$ atmosphere on early Mars could have inhibited $\mathrm{CO}_{2}$ condensation, promoting the possibility of surface temperatures warm enough to accommodate liquid water. Investigators elsewhere pointed out that such a concentration of $\mathrm{SO}_{2}$ would probably not be compatible with a realistic water-rock system. ${ }^{2}$ As an alternative, we now investigate the radiative properties of carbony ${ }^{1}$ sulfide (OCS), which can be expected to coexist more benignly with water and surface rocks. Preliminary findings are that OCS can produce the desired radiative results more effectively than $\mathrm{SO}_{2}$ on a per-molecule basis. Some OCS sources and sinks are also examined.

References

1 Yung, Y.L., Nair, H. \& Gerstell, M.F. (1997). Icarus 130, 222-224.

${ }^{2}$ Murphy, W.M. Bass, D.S. (1998). Bull. Amer. Astron. Soc. 30, DPS 11.03 .

\section{Centrifugation stimulates the immune response of macrophages}

R.K. Globus $\bullet$ NASA Ames Research Center, M.L. Delute $\bullet$ NASA Ames Research Center; San Francisco State University, J.-H. Yu • NASA Ames Research Center; San Francisco State University, S.L. Weinstein $\bullet$ San Francisco State University

The vertebrate immune system is adversely affected by spaceflight. Macrophages are critical sentinels of the immune system and may influence immune responses to spaceflight in vertebrates. During space- flight, cultured macrophages produce more cytokines in response to challenge by lipopolysaccharide (LPS), a component of bacterial membranes and a potent stimulus for macrophages (SK Chapes, J. Leukoc. Biol. 1992, 52, 104). We know little about how gravity affects macrophage function and therefore we used centrifugation to study the influence of hypergravity on macrophage activation. RAW264.7 macrophages were treated with $1 \mu \mathrm{g} / \mathrm{ml} \mathrm{LPS}$, then incubated at $37^{\circ} \mathrm{C}$ in $5 \%$ $\mathrm{CO}_{2}$ in the 1-ft. diameter centrifuge for cell culture (NASA-ARC) and centrifuged to achieve between 2.5 and $50 \times$ gravity $(\mathrm{G})$. Control samples treated with LPS were maintained in an adjacent tissue culture incubator with environmental controls matching those of the centrifuge. After 20 hrs of incubation, conditioned media were collected to measure the inflammatory mediators, nitric oxide (NO), prostaglandin E2 $\left(\mathrm{PGE}_{2}\right)$, TNFa, and IL-6. We found that the LPS-stimulated levels of all these products were elevated in centrifuged samples compared to $1 \mathrm{G}$ controls. At $50 \mathrm{G}$, release of $\mathrm{NO}, \mathrm{PGE}_{2}, \mathrm{TNFa}$ and IL6 increased by $72 \%, 32 \%$, $43 \%$ and $360 \%$, respectively. Centrifugation at $10 \mathrm{G}$ also stimulated release of this products. Cell number was not affected by $50 \mathrm{G}$ centrifugation. We conclude that centrifugation stimulates the immune response of macrophages to bacterial LPS and that this effect is not mediated by increased proliferation. Thus, centrifugation is a useful experimental model to study the inflammatory responses of macrophages to altered gravity environments.

\section{Scientific strategy for the exploration of Mars \\ Ronald Greeley $\bullet$ Arizona State University}

More spacecraft have been sent to Mars than any other planet. However, nearly two-thirds have failed, emphasizing the risks in planetary exploration. Successes include the first spacecraft visit (Mariner 4, 1964), the first geological reconnaissance (Mariner 9, 1971), and the first successful landings (Viking 1976). The Mars Pathfinder (1996) lander marked the successful return to Mars after a two-decade hiatus in exploration; concurrently, the recognition of meteorites from Mars had a significant influence in Mars exploration. Currently in orbit are Mars Global Surveyor and Mars Odyssey; discoveries from these missions reveal that: (1) Mars once had a magnetic field; (2) its surface is dominated by volcanic and active aeolian processes; (3) the primary surface materials are mafic silicates; and (4) past and present environments are conducive for organic evolution and could include nearsurface liquid water in the recent geological past.

An assessment of Mars exploration shows that discoveries commonly occur when new areas are seen (either geographically or in higher resolution) or new instruments are flown (such as the magnetometer and the laser altimeter). The Mars meteorites demonstrate the critical role of samples when they are available for study in Earth laboratories.

The failures of the Mars Climate Orbiter and the Mars Polar Lander in 1998 caused a reorganization of the Mars Program, and the formalization of a long-term science rationale. The latter was achieved through the Mars Exploration Program Analysis Group (MEPAG), an international committee chartered by NASA. MEPAG codified the overarching exploration goals and prioritized the scientific objectives and investigations needed to meet those goals (see http:// mmolib.jpl.nasa.gov/mepag/html [username: mepag, password: gapem]). Building on goals related to the search for life, understanding Mars' climate, and assessing potential resources, MEPAG has refined these goals and defined the means to meet them. Objectives for the life goal include determining whether life exists today and whether life existed in the past, as well as assessing the extent of prebiotic chemical evolution. Objectives for meeting the "climate" goal include characterizing present and past climate and related processes. The "geology" goal has objectives related to determining the evolution of the surface and of the interior, while the "preparation" goal includes determining potential resources on Mars and experiments to demonstrate engineering and other spacecraft systems. Each objective is prioritized and described by investigations and specific measurements needed for fulfillment. To first order, all elements of the plan have been prioritized and an assessment made of the technology readiness for space flight. Although implementation of the full plan will require decades, the plan 
has been developed independent of a specific schedule. Rather, the prioritizations indicate the sequence and the level of importance of each investigation.

The overall strategy for Mars Exploration includes searching (i.e. remote sensing), in situ exploration (e.g. landers/rovers), and sampling (return of materials to Earth), in a continuing, iterative process. A key aspect of the strategy is the inclusion of Mars Scouts, which are missions led by science Principal Investigators who form a team typically involving NASA centers and aerospace industry for projects that complement the primary missions and provide flexibility to the overall program.

The implementation of the Mars Exploration Program will be international, with near-term missions to include NASA's Mars Exploration Rovers (2003), Japan's Nozomi orbiter (2003) the European Space Agency Mars Express (2003), NASA's Mars Reconnaissance Orbiter (2005), and the French CNES Premier orbiter and Netlander mission (2007), as well as other potential projects, leading to the eventual return of samples to Earth from well-characterized sites.

\section{A thin meteorite layer protects bacterial spores in space \\ Gerda Horneck $\bullet D L R$, Institute of Aerospace Medicine, Petra}

Rettberg $\bullet D L R$, Institute of Aerospace Medicine

Meteorites may be natural vehicles for transporting resistant life forms, such as bacterial spores, through space. To tackle the question of whether and to what extent soil or rock material may protect bacterial spores against the harsh environment of space, especially solar ultraviolet (UV) radiation, we have exposed spores of Bacillus subtilis to space (i) unprotected, (ii) under a thin filter of clay, and (iii) mixed with different soil, rock or meteorite powders. Exposure was done in the BIOPAN facility of the European Space Agency onboard the Russian Earth-orbiting Foton satellite. After about 2 weeks in space, the survival was tested from the number of colony formers. Unprotected spores in layers open to space or behind a quartz window, even behind a thin layer of clay, survived barely, if at all, the space trip. Mixing the spores with powder of clay, rock or meteorites increased the survival rate by five orders of magnitude. Up to $100 \%$ survival was reached in $1 \mathrm{~cm}$ cubes of soil mixtures containing spores in concentrations comparable to that in natural soil. These data confirm the deleterious effects of extraterrestrial solar UV radiation. However, they suggest that in a scenario of interplanetary transfer of life, small rock ejecta of a few $\mathrm{cm}$ in diameter could be sufficiently large to protect bacterial spores against the intense insolation.

\section{Alternative trajectories for life on other worlds: lessons from the evolution of life on Earth}

Louis Irwin • University of Texas, El Paso, Dirk Schulze-Makuch • University of Texas, El Paso

The history of life on Earth reveals a number of themes relevant to the possible course of life on other worlds, including: (1) the origin of life relatively quickly under permissive conditions, (2) the conservation of form and function under static environmental conditions, (3) the preponderance of microscopic over macroscopic forms, and (4) the inexorable growth in complexity with time for a diminishing fraction of the total biomass. Assuming that universal laws govern the higher-order organization of matter and energy, several plausible predictions about life on other worlds can be set forth. First, life is probably widespread throughout the universe because the opportunities for its origin are so numerous. Secondly, the vast majority of biomass on other worlds is probably subsurface, as on Earth, where stabilizing selection favors survival in stable environments. Thirdly, extraterrestrial life is predominantly microscopic though not necessarily simple, since life on Earth required over 2 billion years and major planetary transformations to evolve beyond the complexity of unicellular eukaryotic forms. Finally, in rare instances where planetary history has been sufficiently long and favorable, the complexity of life may have evolved to a more elaborate and technologically sophisticated degree than on Earth, in view of the complexity achieved after 4 billion years of life on Earth.

\section{Early eukaryotic diversification}

Emmanuelle J. Javaux $\bullet$ Harvard University, Andrew H. Knoll $\bullet$ Harvard University, Malcolm R. Walter $\bullet$ Macquarie University, Australia

Molecular phylogeny and biogeochemistry indicate that the eukaryotic clade differentiated in the Archean. The presence of C27-C29 steranes in $2.7 \mathrm{Gyr}$ old kerogens provides a minimum age for the appearance of protists. In the Paleoproterozoic, steranes, large spheroidal microfossils, and rare macrofossils document early eukaryotic participation in marine ecosystems. Process-bearing acritarchs diversify in the late Mesoproterozoic and multicellular red algae appear in the early Neoproterozoic. The major environmental change in atmospheric oxygen $2 \mathrm{Ga}$ might have triggered the evolution of photosynthetic eukaryotes, long after eukaryotic heterotrophs. We have shown recently that the early eukaryotic diversification of early eukaryotes began earlier than previously reported (Nature 2001, 412, 66-69). Indeed, early Mesoproterozoic shales from the Roper Group in Australia host a variety of marine protists including cytologically sophisticated organisms that lived in ecologically differentiated communities. Criteria can be developed to identify eukaryotic cells in the early fossil record: the presence of processes or other surface ornamentation; wall structure, ultrastructure, and microchemistry; and the presence of excystment structures. Such research will help us to fill in the gaps between the earliest records of protists and their diversification recorded in younger Proterozoic rocks.

\section{The 2002 Leonid multi-instrument aircraft campaign} Peter Jenniskens $\bullet$ SETI Institute, Major Steven J. Butow $\bullet 129$ th RSQ, USAF

This is to call your attention to an airborne campaign being organized to study the 2002 Leonid meteor storms with support from NASA's Astrobiology and Planetary Astronomy programs. Two intense showers are expected to peak at around 04 and 10 UT, November 19, 2002. These will be the last meteor storms visible in our lifetime. An airborne platform is being made available to view both storms above clouds and above most of the scattered moonlight. The aircraft offers upwardlooking windows for near-UV, visible, near-IR and mid-IR spectroscopy and imaging, and the potential to deploy other remote sensing technologies such as sub-millimeter spectroscopy and lidar. Astrobiology researchers are invited to participate in these missions by contributing experiments complimentary to ongoing efforts. A brief summary will be given of the working conditions in the aircraft, the observing conditions during the upcoming meteor storms, and the experiments performed during past missions.

\section{Ice bacterial abundance, activity and motility observed at subzero temperatures in Arctic sea and lake ice}

Karen Junge $\bullet$ University of Washington, Seattle, David M. Allen $\bullet$ University of Washington, Seattle, Hajo Eicken $\bullet$ University of Alaska, Fairbanks, Jody W. Deming • University of Washington, Seattle

The presence of liquid water often preserved in a frozen state on planets, moons, and comets is considered essential to life. We studied wintertime Arctic sea ice as an important component of the Earth's cryosphere to understand constraints on bacterial parameters at extremely low temperatures, using destructive (bulk analysis of melted samples) and non-destructive methods (fluorescent staining of cells within the 3-D network of brine inclusions). Such observations have revealed the presence of active cells in the coldest ice horizons $\left(-20^{\circ} \mathrm{C}\right)$ and that surfaces are important for the activity of these bacteria. Here we describe adaptation of our methods to high-magnification imaging of bacteria within fluid inclusions of Arctic lake ice under in situ conditions $\left(-5^{\circ} \mathrm{C}\right)$ and to examination of bacterial motility at subzero temperatures. Our preliminary results showing motility of a marine bacterium, Colwellia strain $34 \mathrm{H}$, to $-10{ }^{\circ} \mathrm{C}$, suggest that the temperature limit for motility may be much lower than previously considered. Furthermore, lake ice contains fewer and narrower liquid inclusions, implying reduced fluid exchange and possibilities for bacteria to move, compared with sea ice. Ultimately, we aim to determine the 
lower-temperature limit for motility and other measures of activity to better inform considerations of microbial life in frozen environments on Earth and possibly elsewhere.

\section{Additional evidence from molecular clocks for the Precambrian colonization of land by plants}

Natalie Kardos • Pennsylvania State University, S. Blair Hedges • Pennsylvania State University

The colonization of land by plants was an event that led to major changes in the biosphere. According to the fossil record, this colonization occurred in the Phanerozoic, over 500 million years ago (Ma). However, recent research in our laboratory using molecular clock methods and large numbers of genes has yielded earlier dates, with green algae diverging from the land plant lineage around $1000 \mathrm{Ma}$ and mosses diverging from vascular plants around $700 \mathrm{Ma}$. An early colonization of land by plants raises the possibility that plants impacted the environment of the late Precambrian, including the snowball Earth events and the evolution of animals. To pursue this further, we have increased the size of our molecular data sets and have added another primitive plant, the liverwort Marchantia. The results provide additional support for the Precambrian colonization of land by plants.

\section{Habitable zones around stars and the search for extraterrestrial life \\ James F. Kasting $\bullet$ Pennsylvania State University}

The habitable zone, or HZ, around a star is defined as the region in which an Earth-like planet can maintain liquid water on its surface. The continuously habitable zone, or $\mathrm{CHZ}$, is the region that remains habitable for some finite period of time. These definitions remain useful despite the fact that life may exist in subsurface liquid water regimes (on Mars and Europa, for example) because only surface life can be remotely detected through its effect on a planet's atmosphere.

Climate models can be used to estimate the boundaries of the habitable zone. The inner edge of the $\mathrm{HZ}$ is determined by loss of water via the "moist greenhouse effect" (a variant of the runaway greenhouse effect), while the outer edge is determined by the condensation of $\mathrm{CO}_{2}$ to form clouds and polar caps. The inner edge of our Sun's HZ is conservatively estimated to be at $\sim 0.95 \mathrm{AU}$ but may be further in if water clouds produce a negative feedback on surface temperature. The outer edge of the Sun's HZ is probably at least $1.7 \mathrm{AU}$, i.e. well beyond the orbit of Mars, and may be even further out if $\mathrm{CO}_{2}$ clouds tend to warm a planet's surface or if other greenhouse gases (e.g. $\mathrm{CH}_{4}$ ) provide significant warming. This relatively wide $\mathrm{HZ}$ is a consequence of climate buffering by the carbonate-silicate cycle, which is expected to occur on tectonically active planets like Earth. Mars lacks tectonic activity and, hence, cannot maintain a warm, stable climate. Models that include this negative feedback suggest that relatively broad CHZs exist around stars from at least F0 to K5. Thus, the chances of finding other habitable planets appear to be good, provided that planets themselves are commonplace.

Perhaps more interesting is the question of whether we can detect such planets and look for life on them. NASA's proposed Terrestrial Planet Finder (TPF) mission should eventually do just that. TPF could be either a single, 8 -meter telescope that operates in the visible/nearinfrared or an 80-meter baseline interferometer that operates in the thermal infrared. Ongoing design studies should help to delineate the advantages and disadvantages of each approach. If either one of these designs proves feasible, it could be possible within the next 12-15 years to detect Earth-sized planets around stars out to a distance of $\sim 15$ parsecs and to characterize their atmospheres spectroscopically. Both wavelength regions contain absorption features that may be indicative of both habitability and habitation. In the visible/near-IR, potentially detectable species include $\mathrm{O}_{2}, \mathrm{O}_{3}, \mathrm{H}_{2} \mathrm{O}$, and possibly $\mathrm{CO}_{2}$. $\mathrm{CH}_{4}$ is not observable at modern terrestrial concentrations but could be observed in a weakly reduced, early-Earth-type atmosphere. In the thermal IR, the detectable species include $\mathrm{CO}_{2}, \mathrm{H}_{2} \mathrm{O}, \mathrm{O}_{3}$, and (early-Earth) $\mathrm{CH}_{4}$. Both $\mathrm{O}_{2}$ and $\mathrm{O}_{3}$ are considered to be strong, but not definitive, indicators of life. $\mathrm{CH}_{4}$ by itself is a more ambiguous bioindicator. Ultimately, a second-generation TPF instrument could provide even better evidence for life by looking for the simultaneous presence of $\mathrm{O}_{2}\left(\right.$ or $\left.\mathrm{O}_{3}\right)$ and reduced gases.

\section{Mantle redox evolution and the rise of atmospheric $\mathrm{O}_{2}$ Jim Kasting • Pennsylvania State University, H.D. Holland $\bullet$ Harvard University \\ Why did atmospheric $\mathrm{O}_{2}$ rise around $2.3 \mathrm{Ga}^{1}$ when cyanobacteria appear} to have arisen at least $400 \mathrm{Myr}$ earlier $?^{2}$ One possibility is that the volcanic sink for $\mathrm{O}_{2}$ was initially larger and that the upper mantle became progressively oxidized as hydrogen escaped to space. ${ }^{3}$ Kump et $a l .{ }^{4}$ calculated the change in mantle oxygen fugacity, $\mathrm{fO}_{2}$, required to trigger the $\mathrm{O}_{2}$ rise. They found that a decrease in $\mathrm{fO}_{2}$ by $2 \log$ units would be required to flip the system from oxidizing to reducing. However, data on the $\mathrm{Cr}$ and $\mathrm{V}$ content of ancient basalts ${ }^{5,6}$ rule out a change of this magnitude. Catling et al. ${ }^{7}$ proposed a related model in which the continents became more oxidized; however, the mechanism by which this happened was not specified.

A recent reanalysis of volcanic gas data by Holland ${ }^{8}$ could change this story. Volcanic gases appear to be significantly more $\mathrm{H}_{2}$-rich than had been previously thought. A decrease by as little as a factor of 2 in $\mathrm{fO}_{2}$ may be sufficient to flip the system from oxidizing to reducing. Thus, the original mantle redox theory may indeed be correct.

\section{References}

1 Farquhar et al. (2000). Science 289, 756.

2 Brocks et al. (1999). Science 285, 1033.

${ }^{3}$ Kasting et al. (1993). J. Geol. 101, 245.

${ }^{4}$ Kump et al. (2001). G3 on-line 2.

${ }^{5}$ Canil (1997). Nature 389, 842.

${ }^{6}$ Delano (2001). OLEB 31, 311.

${ }^{7}$ Catling et al. (2001). Science 293, 839.

${ }^{8}$ Holland, GCA (submitted).

\section{Self-ligation of the hairpin ribozyme under freezing and dehydration conditions: a possible prebiotic reaction}

Sergei A. Kazakov • Somagenics, Inc., Brian H. Johnston •

Somagenics, Inc.

The RNA world hypothesis, that early in prebiotic evolution both the information-carrying and catalytic requirements for complex, selfreplicating entities were fulfilled by RNA, has a major difficulty: under an assumed "wet and warm " prebiotic environment, the RNA backbone is chemically unstable, undergoing a cleavage reaction that is accelerated by the presence of di- or multivalent metal ions, the very cofactors that are usually considered necessary for RNA catalysis. Unchecked, this reaction would limit the level of RNA polymer complexity that could be achieved by random processes. We have found that RNA molecules containing the naturally occurring hairpin ribozyme can undergo fast and efficient self-ligation upon freezing or dehydration. This reaction is not dependent on the presence of di- or multivalent metal ions. Freezing could greatly reduce random degradation while permitting increases in complexity through the ligation and recombination of RNA fragments. Freezing water as well as dehydrating conditions may have been available on the early Earth, and may exist even now on Europa and subterranean Mars, possibly providing suitable conditions for the evolution of complex RNA molecules.

\section{The coronagraphic option for a terrestrial planet} finder

Steven Kilston $\bullet$ Ball Aerospace \& Technologies Corporation, Roger Linfield $\bullet$ Ball Aerospace \& Technologies Corporation

Based on the just-completed Architecture Study of design options for the Terrestrial Planet Finder (TPF) space mission, a new large-optics, visible-wavelength coronagraph concept has been developed and evaluated. With considerable promise for both planet-finding and other astrophysics projects, the main goal for this TPF concept is to identify all Earth-like planets in the habitable zones of 150 nearby Sun-like stars. 
It will also be able to measure the concentrations of a few key atmospheric molecules, including molecular oxygen, for many of those planets, in order to detect an environment hospitable to life, or even life's actual presence there.

Currently in a technology development phase, TPF's overall architecture and detailed performance capabilities will be chosen based on future scientific information about the frequency and types of planetary systems, fresh insights into planetary evolution and potential indicators of habitability, and improved evaluations of cost and technology maturity. We describe the properties of Ball's coronagraph concept, and the types and concentrations of biomarkers it can detect in its search for life on other planets.

\section{Early oceans: cradles of life or death traps?}

Paul Knauth • Arizona State University

$\mathrm{Cl}$ is a volatile which follows the water, so it is likely that outgassed planetary seas are originally highly saline. Life either originates and evolves in very salty solutions or it is restricted to planets that differentiated to form continents where fresher waters could develop from distillation/precipitation of seawater and where huge amounts of evaporatively precipitated salts could be gradually sequestered to allow dilution of the parent seas. The salinity history of hydrospheres on other worlds is a crucial issue if optimal salinities are required for the origin and the early evolution of life. The idea that life originated in the early (very salty) ocean on Earth is actually an unexamined assumption currently guiding the search for life elsewhere. Depositonal environments for most of the microfossil occurrences in Precambrian rocks are actually not well understood and intense searching in specifically nonmarine environments has been limited. Current approaches for developing a microfossil and/or geochemical record of early life in nonmarine environments on Earth include studies of paleokarsts, paleocaliche, paleosols, secondary silica in weathered silicic volcanic rocks, and isotopic studies of cherts housing known microfossils. At present, the marine versus non-marine nature of early evolution is unknown, but this is an important issue as we "follow the water" elsewhere.

\section{In vitro selection of peptide-dependent and protein- dependent ribozymes}

Scott M. Knudsen • University of Texas, Michael P. Robertson • University of California, Santa Cruz, Andrew D. Ellington • University of Texas

During a transition from an RNA world to an RNA/protein world, it is likely that ribonucleotide catalysts assembled peptides to serve a function. Peptides could stabilize a catalytic fold, or participate directly in catalysis; thus the ribozyme becomes dependent on its peptide effector. The use of peptides, and ultimately proteins, as catalytic biomolecules could serve the dual roles of broadening the scope of catalysis and of regulating existing catalysts. Previously, effector-dependent ribozymes have been created that are dependent on oligonucleotides, small molecules, and proteins, using rational design, in vitro selection, or a combination thereof. We have used in vitro selection to generate peptide-dependent ribozymes utilizing a pool containing a randomized "effector domain" appended to the catalytic core of the L1 ligase. We have selected for a ribozyme that is activated several thousand fold in the presence of the HIV-1 Rev peptide. This ribozyme is also activated $\sim 3500$ fold by the Rev protein. Optimized clones from a doped selection show activation by Rev peptide of up to 35000 , with increased specificity against Rev protein and other arginine-rich motif peptides. Attempts to optimize ribozymes for activation by the Rev protein yielded clones with faster effector $(+)$ rates, however lower activation profiles due to increased background (effector $(-)$ ) rates.

\section{Dental plaque as a microbial mat}

\section{Paul E. Kolenbrander $\bullet$ National Institute of Dental and Craniofacial} Research

Human dental plaque consists of at least 500 species of densely packed bacteria. Although the temporal order and collective identity of the bacterial species that colonize the enamel surface after professional teeth cleaning is well documented, little is known about the arrangement of species that contribute to the architecture of dental plaque. Temporal order and organized architecture require communication among the participants. The mechanisms of communication within plaque communities include cell-to-cell contact, metabolite exchange, environmental stimuli, and genetic signaling, which may all result in gene-regulation responses by the bacterial community and the host. Successful colonization of a surface in the flowing oral environment demands adherence followed by community formation, as in microbial mats. Oral biofilm communities are subjected to constant abrasive action and shear forces of salivary flow and receive nutrients from the host's diet, glandular secretions, and serum. Some bacteria such as Streptococcus gordonii can grow on saliva independently of other bacteria; other species such as Streptococcus oralis and Actinomyces naeslundii cannot. However, when the latter two species are co-cultured in biofilms with saliva as the sole nutrient source, both species flourish giving an excellent example of a mutualism. Cell-to-cell contact, called coaggregation, is likely to be critical in these mixed-species communities. Of the more than 1000 strains of oral bacteria tested, each strain coaggregates with a defined set of partner organisms. Partnership is not random; many strains have identical sets of partners, providing further evidence that the range of partnerships is highly organized. To locate and identify partners in mixed-species biofilms, fluorescent in situ hybridization and fluorescent antibodies to cell-surface epitopes are used in conjunction with confocal laser microscopy. Gene regulation after cellular contact and signaling between cells are thought to be key in the establishment and consequences of these partnerships. Advances in understanding community architecture in the oral ecosystem will be applicable to many mixed-species microbial communities.

\section{Cosmology and life}

Mario Livio $\bullet$ Space Telescope Science Institute

Recent findings in cosmology and their implications for the emergence and development of life in the universe will be reviewed. In particular, the concepts of dark matter, dark energy, and cosmic star formation history will be discussed, and their relation to the existence of intelligent life will be explored. Links between stellar and biological evolution will also be investigated.

\section{Tales from the outer limits of astrobiology: Titan and giant planets (near and far) \\ Jonathan Lunine • University of Arizona}

Astrobiology's favorite targets are Mars, Europa, the Earth itself, and putative Earths lying in wait around other stars. (One might add meteorites and interplanetary dust particles to the list, though the vast bulk of this material is collected in the Earth environment.) At the outer limits of "respectable" astrobiological targets lie Titan and giant planets.

Titan is the Mercury-sized moon of Saturn with an atmosphere four times denser at its surface than the atmosphere of the Earth. While the bulk of the atmosphere is nitrogen, a few percent is methane. At almost 10 times Earth's distance from the Sun, Titan's lower atmosphere is cold in spite of its mild greenhouse effect - the surface is at $95 \mathrm{~K}$. The chemical conversion of methane (with nitrogen) into suites of hydrocarbons and nitriles, which in turn condense and sediment to the surface as liquid and solid, makes Titan's surface a repository of interesting organic molecules generated in the almost complete absence of water. Once in a while impacts scar the surface of Titan, and melt portions of the crust, which then take upwards of thousands of years to refreeze. While this timescale is short geologically, it is long chemically, and the introduction of hydrocarbons and nitriles to "warm ponds" of liquid water ought to generate at least amino acids, carboxylic acids, and perhaps lead to interesting stereochemical effects such as amplification of right- or left-handed chiral excesses. Moreover, once the water freezes these products are protected from ultraviolet and cosmic ray modification for geological time, and preserved in a cold environment that should slow the degradation of the record of stereoselectivity. 
Could an organics chemistry package find on Titan the well-preserved remains of interesting pre-biotic evolution? We must await CassiniHuygens to further inform this speculation.

The giant planets of our own solar system are well studied and hence provide a stepping-stone to understanding the properties of giant planets around other stars. Unlike extra-solar Earths we now actually have one extra-solar giant planet, HD209458b, whose bulk properties and atmosphere can be directly studied - and understanding Jupiter and Saturn were crucial in unraveling the tale of this beast. Moreover, one can imagine an "easy" Terrestrial Planet Finder mission - which we call the Self-luminous Planet Finder, or SPF - that could take spectra directly of many giant planets in Jupiter-like orbits around other stars. SPF is to TPF as a private sloop is to the Titanic-much easier and much quicker to get underway.

Once we know where they are and what is their nature, the giant planets around other stars become signposts to systems that are and are not habitable. A Jupiter at 1 AU from a Sun-like star precludes "Earth II: The Series" from being played out in that system ... and so might a Jupiter at $3 \mathrm{AU}$. A Jupiter at $5 \mathrm{AU}$ is wonderful, and one at $10 \mathrm{AU}$ would not do much harm, though it might not be as efficient at sending water and other volatiles inward to feed infant Earths. Indeed, returning home to our own habitable planet, we suspect - from the presence of our giant Moon and the low $\mathrm{D} / \mathrm{H}$ value of our ocean - that mighty Jupiter played a mighty big role in making our own planet habitable. And so from the outer limits of astrobiology come some basic insights into how habitable planets arise and where we should look for them.

\section{Sustainability of carbon sequestration in the Duke Forest ecosystem}

Yiqi Luo $\bullet$ University of Oklahoma, Luther White $\bullet$ University of Oklahoma, Evan DeLucia $\bullet$ University of Illinois, Adrien Finzi $\bullet$ Boston University, John Lichter $\bullet$ Bowdoin College, William Schlesinger $\bullet$ Duke University

Significant advances have recently been made in our understanding of the current role of terrestrial ecosystems in the global carbon (C) cycle, using eddy covariance techniques, inversion of atmospheric signals, forest inventory, and simulation models. Estimations from the various approaches suggest that global terrestrial ecosystems sequester up to $3.9 \mathrm{Gt} \mathrm{C}$ per year. By sequestering that amount of $\mathrm{C}$, the terrestrial ecosystems allow the build-up of $\mathrm{CO}_{2}$ in the atmosphere to be only about half the rate of human-induced emission. As international efforts are implemented to stabilize the atmospheric $\mathrm{CO}_{2}$ concentration $(\mathrm{Ca})$ and its associated climate change, it is imperative to quantify the sustainability of terrestrial $\mathrm{C}$ sequestration. Our study first establishes a theoretical framework to define the sustainability based on $\mathrm{C}$ influx and residence times $(T)$. We then estimated $T$ via inverse analysis of multiple data sets from a free-air $\mathrm{CO}_{2}$ enrichment experiment in Duke Forest, North Carolina, USA. The estimated T was scarcely affected by elevated $\mathrm{CO}_{2}$. We used $T$, combined with $\mathrm{C}$ influx, to examine five $\mathrm{C}$ sequestration mechanisms. Although different emission scenarios, photosynthetic acclimation, and $T$ adjustments potentially lead to $\mathrm{C}$ sink stabilization or decline, current evidence suggests that $\mathrm{C}$ sequestration in the forested ecosystem will continue to increase as $\mathrm{Ca}$ gradually increases.

\section{Mass-independent fractionation of oxygen isotopes in Earth's atmosphere \\ James Lyons $\bullet$ University of California, Los Angeles}

Mass-independent fractionation (MIF) has been shown to be an important process in both the early and modern Earth atmospheres. The discovery of MIF in sulfur (S) isotopes in sulfide and sulfate rocks from the Archean (Farquhar et al. 2000) has implications for the level and rise of $\mathrm{O}_{2}$. Modeling of sulfur MIF in the early Earth's atmosphere (Kasting \& Pavlov 2001) is able to place an upper limit on the abundance of $\mathrm{O}_{2}$, although an in-depth study is impossible due to a lack of chemical kinetic data on $\mathrm{S}$ isotope exchange reactions. In the modern Earth's atmosphere MIF of oxygen isotopes, first discovered in ozone (Thiemens \& Heidenreich 1983), has been predicted to be present in nearly all O- bearing molecules (Lyons 2001). The availability of at least some exchange reaction data allows more detailed photochemical modeling for oxygen $(\mathrm{O})$ isotopes. One model prediction is that tropospheric water and stratospheric water differ isotopically in that the former exhibits zero MIF whereas the latter has up to 20 per mil MIF. If confirmed by measurements, this result could be a useful means of assessing shifts in troposphere-stratosphere exchange of water due to global change. More generally, the modeling of MIF in O isotopes provides a good example of the type of exchange processes that will be important for understanding MIF on the early Earth, as well as Mars and the solar nebula.

\section{Planetary systems around other stars}

Geoffrey W. Marcy $\bullet$ University of California, Berkeley

Over 90 planets have been found orbiting other stars, all discovered by the Doppler technique. At least $6 \%$ of nearby stars have Jupiter- and Saturn-sized planets. The mass distribution rises steeply from 8 to 0.3 Jupiter masses, indicating that lower-mass planets of Neptune and Earth mass may be common. Three planetary systems containing multiple planets have been found, and the planets commonly reside in resonances with each other. Unexpectedly, most of the 90 planets (except the closest ones) reside in eccentric orbits rather than circular orbits. This property carries implications for the evolution of planetary systems and the survival of earths.

\section{Extinctions in near time: repairing the damage}

Paul S. Martin $\bullet$ Desert Laboratory, University of Arizona, Tucson Within near time (the last 50000 years) the Earth lost half of its megafauna. Extinction struck mammals mainly our size our larger $(>45 \mathrm{~kg}$ ). We entered near time with about 200 genera of large terrestrial mammals. We arrived in the 21 st century with 100 . Virtually all the extinctions occurred prehistorically.

Globally, the distributions were unequal. Marine mammals, such as whales or dolphins, escaped entirely. On the habitable continents, Australia, North and South America and Madagascar lost over $90 \%$ of their large mammals. Africa and Eurasia suffered least.

Examples of extinctions in North America include mammoths, mastodonts and all our native species of horses and camels. We lost four genera of ground sloths. We lost species of shrub ox, elk-moose, giant peccary, saber-tooth cats, and American subspecies of lion, cheetah, and wolves. South America lost even more including two endemic orders, the toxodons (rhino build) and the litopterns (camel size).

Australia lost dozens of species of marsupials, most larger than living kangaroos and wombats. Madagascar lost elephant birds, two kinds of hippo and eight giant lemurs, the largest the size of a gorilla. New Zealand lost about a dozen species of giant flightless birds, the moas. The West Indies lost dwarf ground sloths. Islands in the Mediterranean lost dwarf hippo, dwarf elephants, and a goatantelope.

What caused the catastrophe? There is no evidence of a cosmic intruder of sufficient size to be lethal. In addition, the timing of the extinctions is sequential, not simultaneous. For the same reason the extinctions cannot be explained by appealing to global climatic change. The Upper Dryas cold snap, for example, correlates well with the time of extinctions in North America but is too late to force Australian extinctions and too early for extinctions in the West Indies, Madagascar, and New Zealand. Furthermore there are many events seen in proxy climatic data preceding and comparable to the Upper Dryas in rate and magnitude of change. If climatic change were involved, extinctions should have coincided with the first glaciations, early in the Pleistocene, rather than coming at the end of a long sequence of cold stadials.

Could Homo sapiens be the Death Star? The timing is right. One big problem in the minds of some is the scarcity of kill sites or other associations linking extinct animals with human remains. But paleontologists do not know the cause of thousands of mammalian extinctions. Even the cause of death of an individual is rarely known.

Whatever the answer, the extinctions of near time terminated the last entire ecosystem in the Americas. Around 13000 years ago when we lost 
our native species of elephants, horses, camels, ground sloths, giant javelina, and many others we lost "Wild America."

What now? Should we reopen the Bering Bridge in a metaphorical sense and restart evolution of our charismatic megafauna? We have already brought back two species of equids, wild horses and wild burros. We began experimenting with reintroduction of camels 150 years ago. Now domestic llamas accompany hikers on the trail. In the absence of mammoths and mastodons, we can study free ranging African or Asian elephants on New World ranges. African rhinoceros were here until ground sloths arrived 5 million years ago. Rhinos are nominees for stepping into the vacated ground sloth niche. Elephants in cut-over Brazilian rain forest should help to disperse large fruits of tropical trees once spread by gomphotheres (extinct proboscideans).

What is the remedy for the extinctions in near time? To restart evolution, initiate Pleistocene Parks as Sergi Zimov has done in Siberia.

\section{Formation of meteorite hydrocarbons from thermal decomposition of the mineral siderite $\left(\mathrm{FeCO}_{3}\right)$ \\ Thomas McCollom $\bullet$ Woods Hole Oceanographic Institution} Two features of the Martian meteorite ALH84001 that have been cited as evidence of extraterrestrial biological activity are the presence of the mineral magnetite with properties resembling those produced by magnetotatic microbes on Earth and polycyclic aromatic hydrocarbons (PAH) such as phenanthrene. Recent experimental and petrologic observations, however, suggest that the magnetite in ALH84001 may have formed abiotically from decomposition of Fe-rich carbonate minerals during a transient heating event. Laboratory experiments were conducted to evaluate the possibility that this process might also result in abiotic synthesis of hydrocarbons. Siderite decomposition in the presence of water vapor at $300^{\circ} \mathrm{C}$ produced a hydrocarbon assemblage composed predominantly of alkylated and hydroxylated aromatic compounds. The results suggest that formation of magnetite by thermal decomposition of siderite on the precursor rock of ALH84001 would have been accompanied by formation of organic compounds, and may represent a source of extraterrestrial organic matter in the meteorite and on Mars. The synthesized compounds are also similar in composition to the organic matter observed in other non-Martian meteorites, suggesting that the underlying processes could have been a significant and ubiquitous source of organic matter in the early solar system.

\section{Amino acid racemization as an indicator for in situ molecular repair processes in permafrost microorganisms}

Gene McDonald $\bullet$ Jet Propulsion Laboratory, Alexandre Tsapin $\bullet$ Jet Propulsion Laboratory, David Gilichinsky $\bullet$ Russian Academy of Sciences

The time span over which dormant organisms can maintain viability in the face of damage to biomolecules from radiation and thermal processes affects the feasibility of transfer of organisms between planets via impact ejecta, as well as the survival time of subsurface permafrost or ice cap biota on Mars. Organisms in sub-freezing environments are often assumed to be metabolically dormant, and thus to be incapable of carrying out intracellular molecular repair processes such as DNA base mismatch repair and the removal of potentially toxic D-amino acids generated by racemization.

We have found (Brinton, K.L.F. et al. 2002, Astrobiology, in press) that the extent of aspartic acid racemization in permafrost samples from Northern Siberia increases linearly up to an age of approximately 25000 years, but at a rate too slow to be consistent with the measured permafrost temperature. We have interpreted these data as evidence of active recycling of D-aspartic acid in Siberian permafrost organisms, indicating that permafrost organisms are capable of repairing some molecular damage even though they appear to be in an overall dormant metabolic state.

We are now extending our study to older core samples from Siberian permafrost, in an effort to determine the maximum age at which the microorganisms can maintain acceptably low $\mathrm{D} / \mathrm{L}$ amino acid ratios.
We must bring back Martian samples

David McKay $\bullet$ NASA Johnson Space Center, Everett Gibson $\bullet$ NASA Johnson Space Center, Kathie Keprta $\bullet$ Lockheed Martin, Houston, Simon Clemett $\bullet$ Lockheed Martin, Houston, Susan Wentworth • Lockheed Martin, Houston

Perhaps the primary science driver of the NASA Mars program is to determine whether life ever existed there. Even the total lack of presentday life and past life on Mars would have profound implications. One lesson we have learned from the search for life in early Earth rocks and in Mars meteorites is that the answers may not be obvious. For Mars, we must do three things: (1) investigate the current environment and reconstruct the past environment through geological studies; (2) send instruments to search for possible biomarkers; and (3) bring back samples for more detailed investigations on Earth. Based on terrestrial experience with Archean terrain, as well as all the Mars missions which have been flown so far, the question of whether Mars did or did not support life will not be easily answered. It has not been possible to get definitive information on possible Martian life from the kinds of instruments that have so far been used in Mars missions. While a new generation of remote sensing and surface analytical instruments is in the development pipeline, it is not clear that they will provide even tentative answers to the question of life, let alone definitive answers. This situation leaves returned samples as our best and perhaps only approach to determining whether life once existed on Mars or may exist today - that is until humans can go there and explore.

\section{Estimates of Europa's heat flow and ice shell thickness: convergence at last? \\ William B. McKinnon $\bullet$ Washington University, St Louis, Paul M. Schenk $\bullet$ LPI, Andrew J. Dombard $\bullet$ DTM, Carnegie Institution of Washington}

We review several quantitative constraints on Europa's shell. Though debated, "pits, spots, and domes" (or lenticulae) bear many morphologic and morphometric hallmarks of diapirism. Regionally, their sizefrequency distributions are approximately unimodal, and spacings have been given as $5-20 \mathrm{~km}$ (the lower end may be less typical). If lenticulae are manifestations of diapirism in an ice shell marginally unstable to convection, and if the diapirs that initiate at the base of the shell can rise through the conductive stagnant lid above (perhaps assisted by tidal heating within the diapirs), then the shell is $7-27 \mathrm{~km}$ thick (McKinnon and Gurnis, LPSC XXX 2058). Stereoderived pit depths of $500 \mathrm{~m}$ imply shell thicknesses in excess of $6 \mathrm{~km}$ if formed by subsurface melting, based on isostasy (Schenk and McKinnon, Jupiter Conf abs., 94-95). This lower limit decreases for a sulfate-rich ocean and clean ice shell, and increases if the shell contains sulfate. Finally, we have applied our extensional/compressional lithospheric instability model (Dombard and McKinnon, Icarus, in press) to the folds discovered at Astypalaea Linae. Minimum heat flows (for lower limit strain rates) of about $75 \mathrm{~mW} \mathrm{~m}{ }^{-2}$ are implied, with a corresponding maximum layer thickness of $8 \mathrm{~km}$ if conductive, greater with a convective sublayer. Taken together, these limits point to a thin but not ultrathin shell.

\section{The wet universe: what we're learning about water beyond our solar system}

Gary J. Melnick $\bullet$ Harvard-Smithsonian Center for Astrophysics

For over three years, NASA's Submillimeter Wave Astronomy Satellite (SWAS) has been conducting pointed and mapping observations toward hundreds of lines of sight within our Galaxy in the lines of several important atoms and molecules, including the ground-state transition of ortho- $\mathrm{H}_{2}{ }^{16} \mathrm{O}$, ortho- $\mathrm{H}_{2}{ }^{18} \mathrm{O}$, and a low-lying transition of $\mathrm{O}_{2}$. Due to the presence of large amounts of $\mathrm{H}_{2} \mathrm{O}$ and $\mathrm{O}_{2}$ in our atmosphere a telescope such as SWAS, situated above the atmosphere, offers the most direct means of studying these bio-critical molecular species. To date SWAS has detected water along most lines of sight observed, yielding a range of inferred abundances spanning a factor of $100000-$ much larger than expected. In particular, while the inferred water abundance 
toward warm-gas regions appears to be in accord with the predictions of chemical models, the water abundance in cold clouds is two to three orders of magnitude below predictions. The observations of $\mathrm{O}_{2}$ are more puzzling still. This talk will review the current state of SWAS observations and our emerging understanding of the processes that produce water in the Galaxy. I will also discuss why these findings do not necessarily bode ill for the evolution of life. A summary of early SWAS results can be found in a dedicated issue of Astrophysical Journal Letters (August 20, 2000).

\section{Geochemical culturing methods link hydrothermal environments with thermophilic communities}

D'Arcy R. Meyer-Dombard • Washington University in St Louis, Everett L. Shock $\bullet$ Washington University in St Louis, Jan P. Amend • Washington University in St Louis

The search for life beyond Earth depends on accurate predictions about the environments and organisms that we can expect to find, using Earth ecosystems as templates. Current studies in astrobiology have targeted several bodies in our solar system, ${ }^{2,3,4}$ whose inhospitable surface climates require serious consideration of subsurface ecosystems that are independent of sunlight as potential oases for life. Indeed, current Earth-based analogs for such astro-ecosystems are rapidly being developed. ${ }^{1}$

Earth ecosystems are most useful as templates for astrobiological ecosystems if the environments are characterized along with the organisms that inhabit them. The hydrothermal systems of Yellowstone National Park offer ideal, unique settings for the investigation of such templates. We have identified three distinct hydrothermal ecosystems as subjects for a thermophilic community survey, conducted entirely within the context of the geochemical environment. These systems range from alkaline to extremely acidic, representing a variety of extreme conditions. Isolates have been obtained in growth media that mimic the geochemical environments, a method which has proven highly successful in our study.

References

${ }^{1}$ Chapelle, F.H. et al. (2002). Nature 415, 312-315.

${ }^{2}$ Chyba, C.F. (2000). Nature 403, 381-382.

3 Jakosky, B. (1996). New Sci. 150, 38-42.

${ }^{4}$ Shock, E.L. (1997). JGR 102, 23687-23694.

\section{Common ancestry of some metabolic enzymes in Escherichia coli \\ Laila Alves Nahum $\bullet$ Marine Biological Laboratory, Sulip Kumar Goswami $\bullet$ Marine Biological Laboratory, Monica Riley $\bullet$ Marine Biological Laboratory}

Previous studies have suggested the predominance of duplication and divergence of ancestral genes in Escherichia coli (review Labedan \& Riley 1999). We have examined some groups of sequence-related metabolic enzymes in E. coli in order to learn more about the different modes of protein evolution. Our results have shown how divergence has taken place in evolution, using common features of reaction chemistry and/or ligand specificity to create catalysts for different kinds of biochemical reactions (Nahum \& Riley 2001). Gcl, IlvI, and PoxB are sequence-related enzymes in different metabolic pathways that illustrate the process of functional divergence from common ancestors. Detailed inspection of the reactions showed that they share a bound cofactor (ThDP), redox prosthetic group (FAD), and the same reaction chemistry (decarboxylation). We have also looked for structural similarities and more distant relatives of these enzymes. Grouping by distant sequence relatedness allows us to collect proteins together that share conserved features, revealing commonalities that probably reflect common ancestry. Understanding the mechanisms of protein evolution helps us to delineate early ancestors, and provide important information on the earliest proteins involved in the origin of life on Earth.

Financial support: Marine Biological Laboratory Astrobiology Institute, and FAPESP, Brazil.

\section{Organic content of Atacama desert soils along the north to south gradient}

Rafael Navarro-González $\bullet$ Universidad Nacional Autónoma de Mexico, Christopher McKay $\bullet$ NASA Ames Research Center, Paola Molina $\bullet$ Universidad Nacional Autonoma de Mexico

The Atacama Desert is one of the driest and oldest deserts of the world that extends across $1000 \mathrm{~km}$ from $30^{\circ}$ South to $20^{\circ}$ South along the Pacific coast of South America. Geological and soil mineralogical evidence suggest that extreme arid conditions have persisted in this desert for about $10-15$ Myrs. We have collected surface soil samples (first $5 \mathrm{~cm}$ layer) from the driest parts of the Atacama Desert $\left(24^{\circ}\right.$ South) to the less arid zones $\left(28^{\circ}\right.$ South) along a transect at about $70^{\circ}$ West. The samples were freeze-dried and then subjected to flash pyrolysis at $750^{\circ} \mathrm{C}$ for $1 \mathrm{~min}$ in a helium atmosphere. The resultant gas fragments were separated by gas chromatography and identified by mass spectrometry. The samples from the most arid zones are basically free of organic matter. No organics are detected using scan mode $(10-100 \mathrm{~m} / \mathrm{e})$ mass spectrometry. Benzene and toluene are just barely detectable using selective-ion-monitoring mass spectrometry. We think that the concentration of organics in desert soils can be used as a proxy for habitability. A lack of organics may indicate an oxidizing environment possibly caused by ultraviolet radiation similar to conditions expected on Mars.

\section{Banded iron formations indicate an oxygenated atmosphere-ocean system since $\sim 3.8 \mathrm{Ga}$}

Hiroshi Ohmoto $\bullet$ Pennsylvania State Astrobiology Research Center Banded iron formations (BIFs) have played an important role in the development of theories concerning the chemical and biological evolution of the early Earth. Most geologists believe BIFs only formed during the periods of $3.8-1.8 \mathrm{Ga}$ and $0.8-0.6 \mathrm{Ga}$, in shallow seas, and under an anoxic atmosphere. However, our geological and geochemical investigations of many major BIF districts in the world (Australia, Canada, USA, South Africa, Finland, and Namibia) suggest entirely different scenarios exist. We conclude BIFs formed throughout geologic history due to their occurrence during the Ordovician, Devonian, and other Phanerozoic ages. Most BIFs, especially Algomatype, formed on deep $(>2 \mathrm{~km})$ seafloor, as a result of the mixing of local bottom seawater with locally discharged submarine hydrothermal fluids. The chemistry of local seawater and hydrothermal fluids determines the mineralogy and chemistry of submarine hydrothermal deposits. For example, oxide-type BIFs form where the bottom ocean water is oxygenrich, carbonate-type BIFs form where the bottom water is anoxic, and sulfide-type BIFs form where sulfate-reducing bacteria are active. The oxygen concentration profile of oceans is related to the oxygen content of the atmosphere. Therefore, the common occurrence of oxide-type BIFs suggests the atmosphere-ocean system has been oxygenated since $\sim 3.8 \mathrm{Ga}$.

\section{micro*scope: a new NAl resource for promoting microbial biodiversity}

David Patterson • Marine Biological Laboratory; University of Sydney, Mitch Sogin $\bullet$ Marine Biological Laboratory

Microbiology lacks diversity in educational resources to attract the next generation of biodiversity specialists. micro*scope is an NAI initiative to provide such resources. The web site includes material in five areas.

1. Classification - a comprehensive hierarchical classification of all genera of prokaryotes and protists.

2. TNS software: Taxonomic Name Server software developed at the MBL manages taxonomic information and links alternative names for the same organism within a hierarchical structure.

3. Internet search tools. micro*scope uses the names of taxa (enhanced using TNS software) to identify biocentric information on the internet and to link formal descriptions, drawings, photographs of microbes, etc. The software aids being used to promote the assembly, interrogation, and compilation of biodiversity information on the internet are in continuing development. 
4. Microbial content: almost 3000 images and descriptions of taxa are available for use by educators and students.

5. Other educational resources: the site contains additional resources to assist teachers. These include talks, copies of general illustrations, animations and Lucid matrix identification guides.

Micro*scope is designed to be a distributed and collaborative web site and contributions by microbiologists and interested educators are welcomed.

\section{Auditing the Earth}

\section{Stuart L. Pimm $\bullet$ Center for Environmental Research and}

Conservation; Columbia University

There was once a time when thoughtful people considered our oceans to be unbounded, the fish stocks within them inexhaustible, the prairies to be a vast wilderness, and the forests to be unending and impenetrable. Is this true today? I will audit the land, the oceans, and the variety of life found there and present seven key numbers.

1. There are six billion of us. That number is rising and (optimistically) will increase by $50 \%$ by mid-century.

2. We use about two-fifths of the land's plant production every year. Most of the stuff we use is from the warmer, wetter half of the planet where plants grow best. What remains is much less suitable. The warm wet places are where forests can grow and the warmest and wettest are the tropical rain forests.

3. Our use of these forests is not sustainable. The world's tropical forests are shrinking by $10 \%$ of their original area per decade.

4. The drier half of the land surface offers less plant production; it is harder to grow crops there. Dry lands are harder to use, provide less food, and are easier to abuse. On over half their area, wind and water erosion has depleted the fertility of the soils. Grazing animals have changed the vegetation in ways that make it less suitable to them.

5. Water is another universal currency. Of the rain that falls over land, the land soaks up two-thirds. We use nearly the same proportion of that (two-fifths) as the plant growth it makes possible. The remaining third runs off the land into rivers, mostly in remote places or as floodwater. We consume $60 \%$ of the accessible runoff each year.

6. About $90 \%$ of the ocean is a biological desert. We use a third of the ocean's production in the remaining $10 \%$. The fraction is not increasing. On the contrary, overwhelming evidence shows that we are destroying the ocean's ability to supply even what we take now.

7. Summarizing human impacts in relation to so much "stuff" leads to succinctness, yet it misses significant details. Life's variety matters. There are probably 10 million kinds of animals and plants, and no more than one in a million - ten - would go extinct per year naturally. In recent history, we have been liquidating species at the rate of 1 in 10000 per year. That's 100 times faster than the natural rate. The rate is now accelerating to between 1000 and 10000 times the natural rate. Nearly a third of all species could be on a path to extinction by the middle of the century.

Humanity's future will be massively different from the past, even if we continue on our present course. We should be informed about present and our likely future for we cannot evaluate our options if we are not.

\section{Population structure in a Philippines hot spring microbial mat}

Stephen Pointing • The University of Hong Kong, Sanjay Nagarkar • The University of Hong Kong, Gavin Smith $\bullet$ The University of Hong Kong, Kimberly Warren-Rhodes $\bullet$ NASA Ames Research Center Surface-dwelling microbial mats within discreet pools of 44.8, 53.3 and $60.1^{\circ} \mathrm{C}$ were sampled using a nested design, in order to gather information on within and between spring variation in population diversity. Denaturing gradient gel electrophoresis (DGGE) will be used to distinguish between PCR amplicons from mat samples obtained using cyanobacteria- and eubacteria-specific primers. Assignment of DGGE bands to individual species will be attempted by sequence analysis.
These data will be compared with estimates of diversity using direct observation of cell morphology within mats and isolation of axenic cultures from enrichment. This presentation illustrates sampling locations and rationale, plus preliminary data from molecular and microscopy studies. Initial findings suggest a unique photosynthetic population structure within these mats, but little within and between pool variation.

\section{A research informed approach to developing an engaging classroom ready curriculum for teaching astrobiology}

Edward Prather $\bullet$ University of Arizona, Timothy Slater $\bullet$ University of Arizona

Effective inquiry-based instruction requires that teachers possess a detailed understanding of the target concepts to be taught. Because astrobiology is a new field of science bringing together many scientific fields of study, it presents a formidable challenge for most $\mathrm{K}-12$ science teachers. The NASA-funded Center for Educational Resources (CERES) Project (URL: http://btc.montana.edu/ceres/) has designed a set of classroom activities and an accompanying Internet course for teaching astrobiology. These activities have been designed to combine on-line data resources from NASA with the student-centered inquiry instructional strategy emphasized in the National Science Education Standards. The activities have been developed and field-tested by precollege science teachers and university faculty. The accompanying asynchronous Internet course is a 15-week, graduate-level course in astrobiology for teachers. The course integrates the NASA Astrobiology Roadmap, the NRC National Science Education Standards, and the astrobiology curriculum supplements available online at URL: http:// btc.montana.edu/ceres/astrobiology. The two main goals of this course are: (1) to provide information on the central concepts related to the field of astrobiology, and (2) to provide experiences with using studentcentered and inquiry-based curriculum materials for teaching astrobiology.

\section{Terrestrial planet formation in the Alpha Centauri system}

Elisa V. Quintana $\bullet N A S A$ Ames; University of Michigan, Jack J.

Lissauer $\bullet$ NASA Ames Research Center, John E. Chambers $\bullet$ NASA Ames, Martin J. Duncan $\bullet$ Queen's University

Some of the 80 known extrasolar giant planets orbit stars which also possess a stellar companion, e.g. 16 Cygni B, Tau Bootis, and 55 Rho Cancri, confirming that planets can form in binary star systems. The radial velocity technique used to discover these planetary companions is able to detect only large (Jupiter-mass) planets, so the existence of Earth-sized planets in binary star systems still remains observationally unconstrained.

Herein, we examine planet formation around each star in the Alpha Centauri A and B binary system, the closest binary to the Sun. Each integration begins with a "bimodal" mass distribution of several large embryos embedded in a disk of smaller planetesimals orbiting a star, and we follow the evolution of the accreting bodies for $200 \mathrm{Myr}-1 \mathrm{Gyr}$. Preliminary results suggest that systems with the initial inclination of the circumstellar disk above 45 degrees to the binary orbital plane cause most of the mass in the disk to rapidly fall into the primary star. When the disk began at a lower inclination, however, the simulations typically produced three to five terrestrial planets within $2 \mathrm{AU}$ of the primary star, and on roughly circular $(e<0.2)$ and roughly coplanar orbits.

\section{The runaway greenhouse effect on Earth and its implication for other planets}

Maura Rabbette $\bullet$ NASA Ames Research Center, Christopher McKay - NASA Ames Research Center, Peter Pilewskie $\bullet$ NASA Ames Research Center, Richard Young $\bullet$ NASA Ames Research Center, Sam Clanton $\bullet$ NASA Ames Research Center

The primary greenhouse gas in the Earth's atmosphere is water vapor. It is an efficient absorber of outgoing long-wave infrared radiation. 
When surface temperature increases, increased evaporation of water from the surface further increases water vapor in the atmosphere, causing more of an atmospheric greenhouse effect. This in turn further increases the surface temperature. If this cycle continues unchecked a runaway greenhouse effect occurs. Venus is thought to be an example where the runaway greenhouse caused the loss of essentially all water from the planet. The runaway greenhouse effect is also a factor in determining the habitable zone of extrasolar planets. Planet habitability is a principal topic in astrobiology, a field for which Ames has major responsibilities. There is observational evidence that the runaway greenhouse effect may occur locally on Earth. Using Earth science satellite data and other in situ data we propose to model the signature of the runaway greenhouse effect. This will allow us to determine whether in fact the runaway greenhouse does occur locally on Earth. If so, we will gain key insights into the Earth's climatology, as well as the processes involved in determining planet habitability.

\section{Embryos, monsters, and the evolution of developmental pathways}

\section{Rudolf A. Raff • Indiana University, Bloomington}

One of the primary goals of modern biology is to forge links between what have in the past been disparate disciplines with little theoretical or experimental connection. The absence of a close tie between evolutionary biology and developmental biology through most of the 20th Century is an obvious case in point. Developmental processes have to evolve in any phylogenetic lineage in order for morphological features to evolve. Furthermore, the organization of ontogeny may well bias the generation of variation upon which natural selection can act. A synthesis of these disparate disciplines has begun, but the question remains as to whether the link is purely contingent, or whether more universal principles exist that produce convergent results. Such possibilities are only now being experimentally investigated. Whether generalizations concerning relationships between developmental processes at the individual level and evolutionary processes at the population level offer useful insights into the evolution of complex organisms on other worlds remains to be seen.

The existence of diverse body plans has posed the problem of how novel body designs have evolved on Earth, and the roles of developmental mechanisms in these grand macroevolutionary experiments. The investigation of evo-devo requires that suitable experimental organisms be found that allow a comparative study of how developmental patterns have evolved, and in what ways developmental mechanisms have been conserved or modified in producing new ontogenies.

In order to do that, my laboratory has focussed on the evolution of development in a congeneric pair of Australian sea urchin species, Heliocidaris erythrogramma and $H$. tuberculata. These two species exhibit an astonishing transformation in embryonic development that has taken place in the approximately 4 million years since their divergence. These evolutionary changes have affected some of the most fundamental processes in development, but in a pair of organisms that are genetically relatively closely related. We are employing several strategies in studying a radical evolutionary transformation in developmental mode of these closely related species. Our approaches include the production of cross-species hybrids between these two species to reveal genes that underlie the evolutionary transformation. The results of hybridization and other experiments indicates that losses in processes that produce individual features of the ancestral feeding larval mode of development of $\mathrm{H}$. tuberculata. However, gains in maternal information governing initial steps of development have occurred in the evolution of the direct-developing form $\mathrm{H}$. erythrogramma. Cross-species hybrids with distantly related independently evolved direct-developing sea urchins suggest that major developmental changes have been convergent in which mechanisms have been exploited in the evolution of similar outcomes.

Secondly, we have identified and cloned sets of candidate regulatory genes, which we are experimentally expressing by microinjection of mRNAs in embryos to produce phenotypes that show the roles of candidate genes in the evolution. Thirdly, we have carried out the microsurgical creation of chimaeras between embryonic tissues of hybrid and parental species to investigate how signals between tissues and their responses have evolved.

The results of our studies show that major changes in early development can occur in a short span of evolutionary time, that a small number of regulatory genes may underlie much of the observed changes in life history and development, and that major evolutionary changes can be strongly convergent in underlying mechanisms.

\section{Bridging nonliving and living matter}

Steen Rasmussen $\bullet$ Los Alamos National Laboratory; Santa Fe Institute, Liaohai Chen $\bullet$ Argonne National Laboratory, Martin Nilsson - Los Alamos National Laboratory, Shigeaki Abe • Los Alamos National Laboratory; Argonne National Laboratory

We demonstrate how an integration of two experimental systems could assemble a proto-organism, as they combine into a single cooperative structure: (i) proto-container, (ii) proto-genes, and (iii) proto-metabolism. This simple system can use energy and nutrients available from its environment, undergo evolutionary change over time, and ultimately die. We present a concrete molecular aggregate that results from thermodynamically downhill processes and that combines: container, metabolism, and genes. As an example, we present experimental progress on a particular set of redox reactions (metabolism), which produce lipids (container) and can be encoded directly in proto-genes. PNA (peptide nucleic acid) strands can be synthesized with a hydrophobic backbone so that they sink into a lipid phase, which would not be possible with, for example, an RNA proto-gene. In brief, upon the irradiation of adenine, the excited state of adenine can be quenched by the phenacyl derivative to generate an adenine cation radical and a phenacyl anion radical. The adenine cation radical is then reacted with adjacent guanine to yield a guanine cation radical, which is eventually scavenged by $\mathrm{HS}^{-}$. Thus the amphiphilic molecules can only be synthesized when the particular AG base sequence is present. As a result of the (autocatalytic) production of lipid molecules the micelles grow, become unstable, and eventually divide.

\section{Where can photosynthesis occur?}

John A. Raven $\bullet$ University of Dundee,UK, Ray D. Wolstencroft $\bullet$ Royal Observatory Edinburgh, UK

What is photosynthesis?: "Building with light" covers a range of biological processes. On Earth, the predominant process involves $\mathrm{CO}_{2}$ reduction, $\mathrm{H}_{2} \mathrm{O}$ oxidation and $\mathrm{O}_{2}$ evolution with production of organic $\mathrm{C}$ :

$$
\begin{aligned}
& \mathrm{CO}_{2}+2 \mathrm{H}_{2} \mathrm{O}^{*}+\geqslant 8 \text { photons }(400-700 \mathrm{~nm}) \\
& \quad \rightarrow\left(\mathrm{CH}_{2} \mathrm{O}\right)+{ }^{*} \mathrm{O}_{2}+\mathrm{H}_{2} \mathrm{O}
\end{aligned}
$$

Other forms of photosynthesis on Earth do not involve $\mathrm{O}_{2}$ production, some do not involve $\mathrm{CO}_{2}$ reduction, and some use a different range of wavelengths.

Significance of photosynthesis on Earth today: Photosynthetic $\mathrm{O}_{2}$ producers on Earth necessarily respire $25-50 \%$ of the reduced carbon they have produced by photosynthesis for growth and maintenance: $\left(\mathrm{CH}_{2} \mathrm{O}\right)+\mathrm{H}_{2} \mathrm{O}+{ }^{*} \mathrm{O}_{2} \rightarrow \mathrm{CO}_{2}+2 \mathrm{H}_{2} * \mathrm{O}$

Even when respiration is taken into account the $\mathrm{O}_{2}$-producers on Earth convert $10^{17} \mathrm{~g}$ of $\mathrm{C}$ from $\mathrm{CO}_{2}$ into organic $\mathrm{C}$ each year in net primary productivity. Rather less than half of this $\mathrm{CO}_{2}$ is fixed in the oceans, and the rest on land.

The organic $\mathrm{C}$ and the $\mathrm{O}_{2}$ produced by $\mathrm{O}_{2}$-producers supports the growth of almost all other organisms on Earth via assimilation of some of the carbon using more carbon in respiration (equation (2)).

Constraints on photosynthesis on Earth today: Equations (1) and (2) show that even with complete absorption of incident solar radiation only $\sim 10 \%$ of the energy in the photons is stored in organic $\mathrm{C}$ in the $\mathrm{O}_{2}$-producer. Comparison of the energy incident on the land and sea surface each year with the global net primary productivity shows that less than $1 \%$ of the energy is used. 
This mismatch is mainly related to the inability of primary producers to harvest the photons incident on the sea or the land surface. In the sea this can be because overlying $\mathrm{H}_{2} \mathrm{O}$ absorbs some of the photons. More generally a shortage of available $\mathrm{N}, \mathrm{P}, \mathrm{Fe}$ or $\mathrm{H}_{2} \mathrm{O}$ prevents the production of enough primary producer biomass to intercept all of the photons or to use them with the theoretical maximum efficiency. Finally, grazing animals or parasitic viruses or microorganisms can limit photon interception and use.

Evolution: photosynthesis in the past: The origin of life on Earth probably involved chemical (chemolithotrophy), thermal (electric storms) or abiotic photochemical (solar radiation) sources of energy for organic syntheses from inorganic precursors. Biologically catalysed photosynthesis evolved as substrates for chemolithotrophic (reducing $\mathrm{CO}_{2}$ using electrons and energy from inorganic chemical reactions) and chemoorganotrophic (growing on organic compounds) organisms were depleted by biological and geological processes. The compounds not useable as substrates for chemolithotrophy or chemoorganotrophy could be used for cell growth when photon energy could be used to assimilate dissolved organic carbon and/or reduce $\mathrm{CO}_{2}$ to produce organic C. Eventually biogeochemical processes resulted in localized depletion of both dissolved organic carbon and reduced inorganic compounds (e.g. $\mathrm{HS}^{-}$) which could, with photosynthetic energy input, reduce $\mathrm{CO}_{2}$ to produce organic $\mathrm{C} . \mathrm{H}_{2} \mathrm{O}$ was a very abundant, but energetically and kinetically recalcitrant, reductant for photosynthesis, and eventually the photosynthetic apparatus changed in evolution such that $\mathrm{H}_{2} \mathrm{O}$ water could be used as an electron donor. The use of $\mathrm{H}_{2} \mathrm{O}$ as an electron donor is correlated with the need for the energy from two photons to be used in transferring each electron from $\mathrm{H}_{2} \mathrm{O}$ to $\mathrm{CO}_{2}$ (equation (1)). The localized accumulation of $\mathrm{O}_{2}$ permitted the evolution of $\mathrm{O}_{2}$-consuming respiration (equation (2)).

It is still not clear as to how global oxygenation occurred, with $\mathrm{O}_{2}$ accumulation in the atmosphere and hydrosphere and an increased oxidation state of the Earth's crust. Local oxygenation could have occurred as early as $3.45 \mathrm{Ga}$, yet global oxygenation did not begin until $2.5 \mathrm{Ga}$. The large-scale accumulation of $\mathrm{O}_{2}$ requires that the $\mathrm{O}_{2}$ consumption in respiration, and in the oxidation of reductants remaining in the deep ocean and on the Earth's surface, and continually emitted from volcanoes and hydrothermal vents be more than balanced by organic $\mathrm{C}$ burial or by $\mathrm{H}_{2}$ production and loss to space. There is still controversy as to the relative roles of reduced $\mathrm{C}$ burial and of reductant loss to space in leading to an oxygenated atmosphere. There is also uncertainty as to how the present $\mathrm{O}_{2}$ content of the atmosphere has apparently been maintained constant to within $10 \%$ or so over the last $250 \mathrm{Myr}$. It is probable that an $\mathrm{O}_{2}$ feedback on the availability of $\mathrm{P}$ for primary productivity is involved.

Photosynthesis on other planets: Earth-like planets (ELPs) could support life "as we know it" within the continually habitable zone, i.e. the distance from the star where liquid water can occur over billions of years. The likelihood of the origin and further evolution of life on ELPs depends on a number of factors, including the occurrence of tectonic activity, and perhaps, a moon which stabilizes the axis of rotation relative to the orbital plane. The likelihood of the evolution of $\mathrm{O}_{2}-$ evolving photosynthesis on an ELP also depends on a number of factors, including the rate of supply of inorganic reductant to the planetary surface. One factor that is not likely to be a constraint is the spectral distribution of radiation from the star, provided there is flexibility in the number of photons which are used in transferring an electron from $\mathrm{H}_{2} \mathrm{O}$ to $\mathrm{CO}_{2}$, with three or four photons per electron needed for ELPs orbiting cooler stars. Even with the maximum likely cloud cover it is likely that an ELP could perform enough $\mathrm{O}_{2}$-evolving photosynthesis to oxygenate the ELP granted Earth-like tectonic activity.

The presence of oxygen makes the remote sensing of $\mathrm{O}_{2}$-evolving photosynthesis via the infrared absorption bands of $\mathrm{O}_{3}$ a possibility. The remote sensing of photosynthesis via the spectroscopic detection of seasonal changes in photosynthetic pigments is also a possibility. However, it must not be assumed that the photosynthetic pigments will have similar spectral properties, and in particular the same long- wavelength absorption limit, as the chlorophylls of land plants on Earth.

\section{GI2, A potential checkpoint protein in Zebrafish: implications for cancer progression and radiation repair \\ Sigrid Reinsch • NASA Ames Research Center, Gregory Conway •} NASA Ames Research Center

We have identified and characterized a potential mitotic checkpoint protein. Checkpoint proteins are crucial for cellular responses to injury, including radiation damage. In a differential RNA display screen we have isolated a zebrafish gene, G12, for which homologs can only be found in DNA databases for vertebrates, but not invertebrates. This suggests that this is a gene required specifically in vertebrates. G12 expression is upregulated at mid-blastula transition (MBT). Morpholino inactivation of this gene by injection into one-cell embryos results in mitotic defects and apoptosis shortly after MBT. Nuclei in morpholinotreated embryos also display segregation defects. We have characterized the localization of this gene as a GFP fusion in live and fixed embryos. Overexpression of G12-GFP is non-toxic. Animals retain GFP expression for at least 7 days with no developmental defects. Interestingly in these animals G12-GFP is never detectable in blood cells though blood is present. In the deep cells of early embryos, G12-GFP is localized to nuclei and cytoskeletal elements in interphase and to the centrosome and spindle apparatus during mitosis. In the EVL, G12GFP shows additional localization to the cell periphery, especially in mitosis. In the yolk syncytium, G12-GFP again localizes to nuclei and strongly to cytoplasmic microtubules of migrating nuclei at the YSL margin. Morpholino injection specifically into the YSL after cellularization blocks epiboly and nuclei of the YSL show mitotic defects while deep cells show no mitotic defects and continue to divide. Rescue experiments in which morpholino and G12-GFP RNA are coinjected indicate partial rescue by the G12-GFP. The rescue is cell autonomous; that is, regions of the embryo with higher G12-GFP expression show fewer mitotic defects. Spot 14, the human homolog of G12, has been shown to be amplified in aggressive breast tumors. This finding, along with our functional and morphological data suggest that G12 and spot 14 are vertebrate-specific and may function either as mitotic checkpoints or as structural components of the spindle apparatus.

\section{Memories of Chuck Klein in the Viking Mission \& how studying left-handed DNA may lead to a therapy for smallpox \\ Alexander Rich $\bullet$ Massachusetts Institute of Technology}

Harold P. (Chuck) Klein was the leader of the active biology team in the Viking Mission to Mars of which I was a member. Participation and planning for that mission as well as the follow-up analysis consumed almost a decade. Those memories will be culled to describe the thinking of that time about searching for extraterrestrial life. In addition, current research will be described in which the nature of left-handed Z-DNA and its binding proteins will be discussed. The surreptitious nature of scientific discovery will be described, as this work may eventually result in a therapy for smallpox.

\section{Hydrous alteration on the surface of Mars: an assessment of the spectral observations}

Steven W. Ruff • Arizona State University, Philip R. Christensen • Arizona State University

With the successful mapping of Mars using the Mars Global Surveyor Thermal Emission Spectrometer (TES), a new spectral data set has been added to existing telescopic and orbital observations. The combined data sets can be used to assess the extent of hydrous alteration of Martian surface materials. In addition to deposits of crystalline hematite of likely hydrothermal origin, TES spectra reveal evidence for $\mathrm{H}_{2} \mathrm{O}$ bearing minerals in the extensive dust deposits that cover large portions 
of the planet. A spectral feature in the 6 micron region that is associated with dust-covered surfaces is likely to be the result of the bending mode of the $\mathrm{H}_{2} \mathrm{O}$ molecule found in clays, zeolites, and other $\mathrm{H}_{2} \mathrm{O}$-bearing minerals. This observed spectral feature is entirely consistent with the $\mathrm{H}_{2} \mathrm{O}$ stretching mode feature at $\sim 3$ microns that has been known for decades. Together, these two spectral features clearly indicate the presence of $\mathrm{H}_{2} \mathrm{O}$-bearing minerals in the dust on Mars that must have formed in the presence of significant amounts of water. In contrast, dark, dust-free regions on Mars display the spectral features of primary igneous minerals such as pyroxene, plagioclase, and olivine. Such minerals are highly susceptible to hydrous alteration yet persist on the Martian surface in significant abundance. These combined observations are clues to the hydrous weathering regime on Mars.

\section{Laboratory calibration studies in support of an ISS exposure experiment and comparison with the diffuse interstellar bands}

Richard Ruiterkamp $\bullet$ Leiden Observatory, The Netherlands, Thomas

Halasinski $\bullet$ NASA Ames Research Center, Farid Salama $\bullet$ NASA

Ames Research Center, Bernard Foing $\bullet$ ESA/ESTEC, The

Netherlands, Pascale Ehrenfreund $\bullet$ Leiden Observatory, The

Netherlands

Polycyclic aromatic hydrocarbons (PAHs) are important interstellar prebiotic molecules. The Infrared Space Observatory (ISO) has provided new insights into the size distribution and the structure of these molecules pointing to a trend towards larger-size PAHs. The infrared spectra of galactic and extra galactic sources have also indicated the presence of PAH structures. This paper reports for the first time the laboratory measurement of the ultraviolet-near-infrared absorption spectra of a representative set of large PAHs that have been selected for a long duration exposure experiment on the International Space Station (ISS). This experiment will test the photostability of large carbonbearing molecules during a long-duration exposure in Earth orbit. Comparison of these laboratory spectra with the spectra of the postflight samples will provide important information on the effects of space radiation on cosmic organic materials.

\section{The AstroBiology Explorer (ABE) MIDEX Mission concept: using infrared spectroscopy to identify organic molecules in space}

Scott Sandford $\bullet$ NASA Ames Research Center, Kimberly Ennico • NASA Ames Research Center, Louis Allamandola $\bullet$ NASA Ames Research Center, Jesse Bregman $\bullet$ NASA Ames Research Center, Thomas Greene $\bullet$ NASA Ames Research Center, Douglas Hudgins • NASA Ames Research Center

One of the principal means by which organic compounds are detected and identified in space is by infrared (IR) spectroscopy. Past IR telescopic and laboratory studies have shown that much of the carbon in the interstellar medium (ISM) is in complex organic species, but the distribution, abundance, and evolutionary relationships of these materials are not well understood. The Astrobiology Explorer (ABE) is a MIDEX mission concept designed to conduct IR spectroscopic observations to detect and identify these materials and address outstanding problems in astrobiology, astrochemistry, and astrophysics. ABE's core science program includes observations of planetary nebulae and stellar outflows, protostellar objects, Solar System objects, and galaxies, and lines of sight through dense molecular clouds and the diffuse ISM. ABE is a cryogenically cooled $60 \mathrm{~cm}$ diameter space telescope equipped with three cross-dispersed $\mathrm{R} \sim 2000$ spectrometers that share a single common slit. Each spectrometer measures one spectral octave and together cover the entire 2.5-20 $\mu \mathrm{m}$ region simultaneously. The spectrometers use state-of-the-art InSb and $\mathrm{Si}: \mathrm{As}$ $1024 \times 1024$ pixel detectors. ABE would operate in a heliocentric, Earth drift-away orbit and have a core science mission lasting $\sim 1.5$ years. ABE is currently under study at NASA's Ames Research Center in collaboration with Ball Aerospace and Technologies Corporation.

\section{Reduced nitrogen compounds in hydrothermal vent systems: a critical evaluation \\ Martin Schoonen $\bullet$ State University of New York, Stony Brook,} Alexander Smirnov $\bullet$ State University of New York, Stony Brook Reduced nitrogen compounds (e.g. $\mathrm{NH}_{3}\left(\mathrm{NH}_{4}^{+}\right), \mathrm{HCN}$, and urea) are far more reactive reagents in prebiotic synthesis reactions than $\mathrm{N}_{2}$. Hence, for prebiotic synthesis reactions, such as amino acid formation, to occur in and around hydrothermal systems reduced $\mathrm{N}$-compounds need to be advected or formed in situ. Here we have examined the in situ formation of reduced $\mathrm{N}$-compounds. Reaction path modeling indicates that ammonium is the stable $\mathrm{N}$-species below $350{ }^{\circ} \mathrm{C}$ in solutions buffered by any of the common mineral buffers, except hematitemagnetite. None of the conditions yield any other reduced $\mathrm{N}$ compounds at concentrations above $10^{-9} \mathrm{M}$. The few available gas data on modern vent systems show, however, that $\mathrm{N}_{2}$ is far more abundant than $\mathrm{NH}_{4}^{+}$. Possible sources for $\mathrm{N}_{2}$ include unreacted $\mathrm{N}_{2}$ dissolved in recharging seawater or mantle-derived $\mathrm{N}_{2}$. We compared molar $\mathrm{N}_{2} / \mathrm{Ar}$ ratios for vent solutions to those for MORB (65) and airsaturated seawater (asw) (36). Furthermore, we compared the concentration of dissolved $\mathrm{N}_{2}$ in vents to that in asw. The data suggest that most $\mathrm{N}_{2}$ entrained in the recharging seawater does not react. Therefore, to provide reduced $\mathrm{N}$-compounds for prebiotic synthesis in and around vent systems it is necessary to : (1) advect the reduced compounds or (2) have a more reactive mineral assemblage on the early Earth.

\section{The search for ancient life: a fossil hunter's notions \\ $J$. William Schopf • University of California, Los Angeles}

The emergent field of astrobiology - an inquiry into the origin and evolution of almost absolutely everything - is difficult and demanding. But the challenges it presents are not due simply to the all-inclusive questions the field addresses. Rather, their root cause, I think, lies in our own deficiencies, in the inadequacies of those of us who are practitioners of the science. Consider, for a moment, the nature of Nature. The natural world is neither entirely physical nor wholly biological, yet in our institutions we divide this world into two great camps - the Physical Sciences and the Life Sciences - populated by tribes of such different backgrounds and divergent interests that they barely speak the same language. On most college campuses the two tribes are even partitioned into separate "homelands" the borders of which, though claimed to be open to all, are actually seldom crossed. Each of us, all products of this system, bears allegiance to one or the other of the two great tribes. And while this tribalism has simplified our lives - learning the ropes in a single discipline being far easier than grappling with many - we each also have paid a price. For astrobiological science, the cost has been high. The real world is a interdependent mix of the physical and life sciences; not one; not the other; but both. Despite our predilections to the contrary, Nature is not compartmentalized! Unless we astrobiologists find ways to better bridge the chasm that separates the sciences, our field will suffer.

Rather than dwell on mistakes that could have been avoided had the chasm between the sciences been better bridged, my goal here is to highlight one aspect of astrobiology - the search for evidence of ancient life - in which (from my admittedly myopic perspective) rigorous interdisciplinary science has paid major dividends.

Over recent decades, the rules for accepting ancient microfossil-like objects as bona fide Precambrian fossils have come to be well established, namely, that such objects be demonstrably biogenic, and indigenous to and syngenetic with the formation of rocks of known provenance and well-defined Precambrian age. Three new techniques have recently been devised to answer the question of biogenicity, the most vexing of the criteria to satisfy. Founded on a firm understanding of the morphology and physiology of extant microorganisms and a large body of data on the maturation of organic matter in geological settings, for the first time these techniques provide a means to correlate directly cellular morphology with organic composition in single Precambrian microscopic fossils. (1) Ion microprobe analyses have been used to measure the carbon isotopic compositions of individual fossils in geological units 
$\sim 850$ - and $\sim 2100$-Myr-old. ${ }^{1}$ (2) Laser-Raman imagery has been used to analyze the molecular compositions of individual cellular fossils and particulate organic matter in 25 geological units ranging from $\sim 400$ to nearly 3500 Myr-old, including representative specimens from each of the three oldest fossiliferous units now known. ${ }^{2,3,4}$ (3) Atomic force microscopy has been used to reveal the submicron-scale structure of the kerogenous components of single Precambrian microfossils. ${ }^{5}$ Taken together, the results obtained demonstrate that the molecular structure of the fossils analyzed varies systematically with metamorphic facies, fidelity of fossil preservation, color of the preserved organic matter, and $\mathrm{H} / \mathrm{C}$ ratios of bulk-sample kerogens - results that not only establish the biogenicity of the kerogenous microscopic fossils analyzed but provide new insights into the chemical changes that accompany organic metamorphism.

Studies such as these require expertise in disparate disciplines and their particular techniques - microbiology and micropaleontology; organic chemistry and biochemistry; stable isotopic and organic geochemistry; laser-Raman spectroscopy and atomic force microscopy. Given this, the reality that astrobiologic science is inherently multidisciplinary, what can be done to help our field? Three suggestions are: (1) institute month-long summer workshops for senior workers new to the field, taught by experienced "old-timers" and designed to produce publishable results. (2) Establish a national facility properly equipped (for laser-Raman imagery, ion microprobe analyses, electron and atomic force microscopy, etc.) and specifically dedicated to an integrated chemical-morphological search for ancient evidence of life. And, over the long-term (3), encourage university-level multidisciplinary education in the sciences. ${ }^{6}$

References

${ }^{1}$ House, C.H., Schopf, J.W., McKeegan, K.D., Coath, C.D., Harrison, T.M. \& Stetter, K.O. (2000). Carbon isotopic composition of individual Precambrian microfossils. Geology 28, 707-710.

${ }^{2}$ Kudryavtsev, A.B., Schopf, J.W., Agresti, D.G. \& Wdowiak, T.J. (2001). In situ laser-Raman imagery of Precambrian microcopic fossils. Proc. Nat. Acad. Sci. USA 98, 823-826.

${ }^{3}$ Schopf, J.W., Kudryavtsev, A.B., Agresti, D.G., Wdowiak, T.J. \& Czaja, A.D. (2002). Laser-Raman imagery of Earth's earliest fossils. Nature 416, 73-76.

${ }^{4}$ Schopf, J.W., Kudryavtsev, A.B., Agresti, D.G., Czaja, A.D. \& Wdowiak, T.J. In preparation.

${ }^{5}$ Kempe, A., Schopf, J.W., Altermann, W. \& Heckl, W.M. In preparation.

${ }^{6}$ Schopf, J.W. \& Hirsch, W.Z. Strategies to foster multidisciplinary teaching and research in a university context. In As the Walls of Academia Are Tumbling Down, eds Hirsch, W.Z. \& Weber, L.E., (London: Economica Press). In press.

Laser-Raman imagery of the oldest fossils on Earth J. William Schopf $\bullet$ University of California, Los Angeles, Anatoliy B. Kudryavtsev $\bullet$ University of Alabama at Birmingham, David G. Agresti - University of Alabama at Birmingham, Andrew D. Czaja $\bullet$ University of California, Los Angeles, Thomas J. Wdowiak • University of Alabama at Birmingham

Laser-Raman imagery is a new non-intrusive, non-destructive technique that can be used to demonstrate a one-to-one correlation between optically discernable morphology and the organic (kerogenous) composition of individual permineralized fossil microorganisms. Made possible by the high sensitivity of Raman spectroscopy to the distinctive signal of carbonaceous matter, such analyses can be used to characterize the molecular composition of organic-walled fossils as small as 1 micron in diameter in polished or unpolished petrographic thin sections, in the presence or absence of microscopy immersion oil, and whether the fossils analyzed are exposed at the upper surface of, or are embedded within, the section studied.

We have used this technique to analyze microscopic fossils and particulate kerogen in 25 fine-grained chert units ranging in age from Devonian to Archean, including the oldest fossils known: filamentous microbes from the $\sim 3375$-Myr-old Kromberg Fm. of South Africa; and colonial spheroids from the $\sim 3430$-Myr-old Strelley Pool chert and cellular filaments from of the 3465-Myr-old Apex chert, both of Western Australia. These studies show that the molecular structure of the organic-walled fossils and particulate kerogen varies systematically with metamorphic facies, fidelity of fossil preservation, color of the organic matter analyzed, and bulk-sample $\mathrm{H} / \mathrm{C}$ ratios - results that not only establish the biogenicity of the fossils studied but provide insight into the chemical changes that accompany organic metamorphism.

\section{Osteoblast cytoskeletal and $\mathrm{PGE}_{2}$ responses to hypergravity \\ N.D. Searby $\bullet$ Stanford University; NASA Ames Research Center, C.R. Steele $\bullet$ Stanford University, R.K. Globus $\bullet$ NASA Ames Research Center}

Bone changes appear to be a serious issue for long-duration spaceflight, and are believed to be in part due to impaired function of bone-forming osteoblasts. Thus, it is important to understand how biomechanical loads influence osteoblast activity. Osteoblasts respond to various mechanical forces, including mechanical strain and fluid-induced shear stress. We used an experimental approach to ask whether hypergravity induces osteoblast cytoskeleton and overall shape changes, and whether the paracrine signaling factor prostaglandin $\mathrm{E} 2\left(\mathrm{PGE}_{2}\right)$ increases as observed with other types of mechanical stimulation. Structural modeling was then performed to ask whether experimentally measured cell shape changes could be predicted based on engineering principles. To experimentally evaluate the influence of gravity, cultured primary fetal rat osteoblasts were grown to near confluence and centrifuged at 10 times gravity $(10-\mathrm{g})$ for 3 hours using the NASA Ames 1-ft diameter centrifuge, with 1-g controls placed in an adjacent incubator. Immediately after centrifugation, the conditioned medium was collected and analyzed for $\mathrm{PGE}_{2}$ content. Microtubules and nuclei were fluorescently labeled and analyzed by confocal microscopy to determine microtubule morphology and overall microtubule network height. Centrifugation led to a three-fold increase in $\mathrm{PGE}_{2}$ production compared with the stationary controls. No major morphological changes were seen in the microtubule network architecture. Microtubule network height was reduced by $15 \%$ relative to stationary controls. The osteoblast structural model, constructed assuming a simplified axisymmetric geometry, material properties from the literature, maintenance of a constant cell volume, and linear elastic behavior, was subjected to centrifugation-induced loads. Modeling results indicated that the magnitude of the measured cell height changes could not be explained directly by differences in gravity acting on the densities of cell components such as the nucleus, but could be explained partly by removing the constant cell volume assumption. In conclusion, $\mathrm{PGE}_{2}$ production increased in a manner similar to other mechanical loads, and osteoblast microtubule network height was reduced due to centrifugation. Modeling has led to the definition of the next important experiments to be performed to understand the role of the cytoskeleton in the response of osteoblasts to hypergravity. (Supported in part by NASA grants NAGW4625 and 99-HEDS-02.)

\section{Environmental effects of large impacts on Mars: implications for river formation \\ Teresa L. Segura $\bullet$ LASP, University of Colorado, Owen B. Toon • LASP, University of Colorado, Anthony Colaprete $\bullet$ NASA Ames Research Center, Kevin Zahnle $\bullet$ NASA Ames Research Center} The impacts of asteroids and comets larger than $100 \mathrm{~km}$ in diameter have left more than 30 craters on Mars. Collisions of such large, energetic objects result in the production of meters thick global rock rain layers, that have temperatures in excess of $1600 \mathrm{~K}$. These global rock melt layers warm the near-surface regolith of the planet considerably, melting subsurface water and keeping the regolith above freezing for decades or even centuries for very large impacts. In addition, large impacts inject into the atmosphere a substantial amount of vaporized water, both from the impactor itself and from the target material on the planet, which will condense and precipitate out. We use a one-dimensional radiative transfer code coupled to a time-marching finite-difference one-dimensional subsurface model to calculate the 
evolution of the atmospheric, surface, and subsurface temperatures following the impact. The model is adjusted for early Martian conditions by reducing the solar constant to $75 \%$ of its present value, and by increasing the post-impact $\mathrm{CO}_{2}$ pressure to 150 mbar. The melted subsurface water, and the injected vaporized water will contribute to a global surface water layer meters or more in depth. The global water layer resulting from a large impact would form rivers and may be responsible for the observed valley networks on Mars.

\section{Non-enzymatic oligomerization on DNA templates containing propynyl pyrimidine nucleobases}

Surajit Sinha $\bullet$ University of California, Riverside, John Chaput $\bullet$

University of California, Riverside, Christopher Switzer $\bullet$ University of California, Riverside

The search for RNA-like molecules capable of sequence-independent replication in the absence of enzymes remains problematic. In the case of RNA, efficient copying of templates using activated monoribonucleotides only occurs with C-rich templates (Orgel, L.E. Origins of life Evol. Biosph. 1993, 23, 285). This circumstance is due to enhanced base stacking of $\mathrm{G}$ monomers and the stability of the $\mathrm{G}: \mathrm{C}$ base-pair. In contrast, uridylate- and purine-rich templates are inefficient in copying reactions. We have recently embarked on a program intended to overcome these problems by fortifying stacking and hydrogen-bonding interactions between nucleobases. 5-propynyl pyrimidines are known to enhance DNA double helix stability by approximately $2^{\circ}$ /residue relative to natural nucleobases (Barnes, T.W., Turner, D.H.J. Am. Chem. Soc. 2001, 123, 4107). We have synthesized hairpin templates bearing propynyl pyrimidine residues to examine their effect on template-directed non-enzymatic oligomerization. After 10 days, we find full length oligomerization products formed efficiently with templates bearing propynyl modifications, whereas the corresponding natural templates gave incomplete reactions. Additionally, we find that phosphodiester bonds formed by the modified templates are predominantly $3^{\prime}-5^{\prime}$ linked.

\section{Nearby young stars and planets}

Inseok Song • University of California, Los Angeles, M.S. Bessell • Australian National University, Australia, B. Zuckerman • University of California, Los Angeles

Nearby young stars are excellent targets for direct imaging detection of cooling planets because young giant planets are hot in youth and hence bright at near-infrared wavelengths. We are involved in a long-term project to find the youngest and closest stars to Earth by using X-ray (RASS) and astrometric (Hipparcos and Tycho) catalogs. We generated a list of nearby young star candidates (of order 1000) and we have observed most of the good targets from the list with the $2.3 \mathrm{~m}$ telescope at Siding Spring Observatory in Australia and with the $3 \mathrm{~m}$ telescope at Lick Observatory in the US. To date, we found that about 150 stars are indeed close to Earth and very young, i.e. showing strong $\mathrm{H} \alpha$ emission and/or Li $6708 \AA$ absorption. In particular, we found the closest young association known to date, the beta Pictoris moving group. These stars share the same space motion as beta Pictoris, the famous nearby star with a very prominent protoplanetary disk. Among the identified young stars, many stars that lack parallaxes may also be members of already known nearby young stellar groups such as the beta Pictoris moving group and the Tucana/Horologium Association. We plan to measure their accurate parallaxes so that their space motions can be determined. We are currently searching for planetary companions around these newly identified young nearby stars with ground-based adaptive optics at Lick and Keck observatories. This research was supported in part by the UCLA Astrobiology Institute and by a NASA grant to UCLA.

\section{The diversity of life in a crystalline salt ecosystem}

John Spear $\bullet$ University of Colorado, Boulder, Ruth Ley $\bullet$ University of Colorado, Boulder, Norman Pace • University of Colorado, Boulder The goal of this work is to describe the organismal composition, structure and physiology of a microbial ecosystem within crystalline salt. Sea salt is crystallized by solar evaporation at the Exportadora de
Sal, in Guerrero Negro, Baja California Sur. Sea water flows through a series of evaporative basins with an increase in salinity until saturation is reached and halite crystallization begins. Several of these ponds are underlined with thick $(10 \mathrm{~cm})$ microbial mats. A microbiological ecosystem occurs whereby crystallized salt, lying in near-saturated salt water, is laden with microbial activity. Exposure of the interior of these large (kgs), wet, endoevaporative salt crystals reveals a multitude of colorful microbiota.

A survey of the stratified microbial communities in these salt crystals is underway by a molecular approach, based on cloning and sequencing of 16S rRNA genes for phylogenetic analyses, to determine the nature and extent of microbial diversity within this ecosystem. Analyses to date indicate the ubiquitous dominance of uncultured organisms of phylogenetic kinds not generally thought to be associated with hypersaline environments. The numbers of different kinds of organisms is dramatic, given that they occur in crystallized salt. The results indicate that the diversity of life is extensive, even in this seemingly inhospitable "extreme" environment.

\section{Novel viruses from extreme environments}

Kenneth Stedman $\bullet$ Portland State University, George Rice $\bullet$ Montana State University, Jamie Snyder $\bullet$ Montana State University, Blake

Wiedenheft $\bullet$ Montana State University, Mark Young $\bullet$ Montana State University, Wolfram Zillig • Max Planck Institute for Biochemistry

Novel spindle-shaped viruses from samples collected from acidic $(\mathrm{pH}<4)$ hot $\left(70^{\circ} \mathrm{C}\right)$ springs in Japan, Iceland, Yellowstone National Park, USA and Kamchatka, Russia have been isolated and characterized. All of the viral hosts appear to be very closely related to Sulfolobus solfataricus, one of the best characterized extremely thermophilic archaea. All of these viruses infect S. solfataricus and contain extrachromosomal double-stranded DNAs. We have determined the complete genome sequence from viruses from Iceland, Japan and Russia and partial sequences from the USA. The viral genomic sequences are related, but are very diverse, reflecting their geographic isolation. Nevertheless, transcription and presumably regulation appear to be similar in the viruses. Essential ORFs are well conserved, whereas ORFs identified as not being essential are less so. Viral proteins with assigned functions appear to be well conserved with one glaring exception. Codon distribution offers insight into virus evolution. Additionally in samples from hot acidic springs in Yellowstone National Park, USA, novel virus morphologies were observed, some of them similar to known viruses of Sulfolobus, all of which have hitherto novel morphologies, and some completely different. Preliminary characterization of these viruses indicates that they are also DNA viruses, but with highly unusual characteristics.

\section{Biotechnology for solar system exploration}

Andrew Steele $\bullet$ Carnegie Institution of Washington, Jake Maule $\bullet$ $C I W$, Jan Toporski $\bullet$ CIW, Victor Parro Garcia $\bullet$ CAB, Carlos Briones $\bullet$ CAB, Mary Schweitzer $\bullet$ Montana State University, David McKay $\bullet$ NASA Johnson Space Center

With the advent of a new era of astrobiology missions to search our solar system for evidence of life we would like to again present a new approach to this goal. We have reviewed the current list of Biotechnology techniques, which are applicable to miniaturization and integration into a combined flight platform. Among the techniques reviewed are:

- the uses of antibodies

- fluorescent detection strategies

- protein and DNA chip technology

- surface plasmon resonance and its relation to other techniques

- microelectronic machining (where applicable)

- nanotechnology (where applicable)

- laboratory on a chip technology (including PCR)

- mass spectrometry

- fluid handling and extraction technologies

- Chemical force microscopy (CFM)

- Raman spectroscopy

- Nanotechnology (i.e. molecular motors). 
We have begun to integrate this knowledge into a single flight instrument approach for the sole purpose of combining several mutually confirming tests for life, organic/microbial contamination, prebiotic and abiotic organic chemicals.

We will present several innovative designs for new instrumentation including pro-engineering design drawings of a protein chip reader for space flight and fluid handling strategies. We will also review the use of suitable extraction methodologies for use on different solar system bodies.

\section{High-speed spectroscopic observation of chemical reaction of carbon within impact-induced vapor clouds}

Seiji Sugita $\bullet$ University of Tokyo, Peter Schultz $\bullet$ Brown University Significant amount of organic carbon is expected to exist in primitive asteroids. However, most organic matter delivered to the Earth's surface by large meteoritic bodies is thought to be destroyed on impacts, particularly in an oxidizing atmosphere. Nevertheless, the consequences of impact-destroyed organic matter are not yet well understood. In order to observe chemical reaction of reduced carbon in an impact vapor within an oxidizing atmosphere, we performed a series of impact experiments using carbon-rich projectiles, high-impedance metal targets, and $\mathrm{N}_{2}-\mathrm{O}_{2}-\mathrm{Ar}$ model atmospheres. The emission spectra of the impacts were observed with high-speed spectrometers. The spectroscopic observation indicates that vaporized carbon readily reacts with atmospheric nitrogen and forms $\mathrm{CN}$ radicals, which are unstable in an oxidizing condition. This experimental result suggests that a carbonrich meteoritic body vaporized in an oxidizing atmosphere may create a reducing local environment, which may help synthesizing organic matter from once destroyed meteoritic organic matter. Such re-synthesis of organic matter may increase the effective survivability of meteoritic organic matter significantly.

\section{Graduate education in astrobiology: producing the first real astrobiologists \\ W.T. Sullivan $\bullet$ University of Washington, J.T. Staley $\bullet$ University of Washington}

Many educational issues have arisen in the emerging field of astrobiology. At the University of Washington (UW) we have focused on graduate education and the question of how best to produce the new generation of astrobiologists. In 1999 we admitted our first students and now have 15 participants with majors across ten departments. There is no "Department of Astrobiology" - students still receive a Ph.D. in their home department's discipline (e.g. oceanography, microbiology, astronomy), but spend about $30 \%$ of their time earning an additional Certificate in Astrobiology. This talk will describe this pioneering program, how it has been shaped and is still being redefined, and lessons learned.

The program aims to produce a student solidly grounded in one discipline, but so exposed to the other disciplines comprising astrobiology that she or he is familiar with the key concepts and "culture" of other relevant disciplines (e.g. allowing the current literature to be read and understood to a large degree), is oriented towards thinking about problems in a more universal scientific way, and is comfortable with, indeed even desirous of, multidisciplinary collaborative projects, especially those at the heart of astrobiology that link the physical and biological sciences.

We achieve this through: (1) specially designed core courses and seminars, (2) an annual hands-on field workshop for both faculty and graduate students (topics have included subterranean microbiology, invertebrate marine biology, paleontology, and choosing a Martian landing site), (3) rotations through labs outside one's own department, (4) graduate research stipends that are attached to the student rather than to the faculty member, and (5) multidisciplinary mentoring and advising as the student chooses and researches his or her Ph.D. thesis.

A natural outgrowth of our program has been a graduate-level textbook, Planets and Life: The Emerging Science of Astrobiology (to appear in 2003), edited by Sullivan and John Baross (UW Biological
Oceanography), with about one-third of the material from UW authors (including our students) and the remainder by other experts. The style (which we also request of all speakers in our seminars) is that the first two-thirds of each chapter should be understandable by anyone with a bachelor's degree in a field relevant to astrobiology, with the remainder becoming more technical.

http://depts.washinton.edu/astrobio

\section{SETI: pulling signals out of cosmic noise \\ Jill Tarter $\bullet$ SETI Institute}

As one legitimate method of attempting to answer the "Are we alone?" question posed by astrobiology, searching for evidence of technological civilizations offers a different, appealing, and popular approach. Today there are a dozen active, privately-funded SETI projects on telescopes worldwide, and SETI forms the basis for the largest distributed computing project ever undertaken.

A recent series of workshops has laid out a roadmap for SETI research for the next few decades. Three different approaches were identified. (1) Continue the radio search; build an affordable array from consumer market components, expand the search in frequency, and increase the target list to 100000 stars. This array will also serve as a technology demonstrator and perhaps enable the international radio astronomy community to realize an array that is 100 times larger and capable (among other things) of searching a million stars. Reinvent ways to conduct radio SETI surveys of the sky. (2) Begin searches for very fast optical and infrared pulses from a million stars and conduct an OSETI survey of the sky. (3) As Moore's Law delivers increased computational capacity, build an omni-directional sky survey array capable of detecting strong, transient, radio signals from billions of stars.

SETI could succeed tomorrow, or it may be an endeavor for multiple generations. We are, after all, a very young technology in a very old galaxy. While our own leakage radiation continues to outshine the Sun at many frequencies, we remain detectable to others. When our use of the spectrum becomes more efficient, and our leakage radiation becomes more noise-like, it will be time to consider deliberate transmissions and the really tough questions: Who will speak for Earth? What will they say? Maybe by then we will be old and wise enough to find some answers.

\section{Voyages through time: an integrated science course for astrobiology}

Jill Tarter $\bullet$ SETI Institute, Edna DeVore $\bullet$ SETI Institute, Jane Fisher - SETI Institute, Yvonne Pendleton $\bullet$ NASA Ames Research Center, Kathleen O'Sullivan $\bullet$ San Francisco State University, Margaret Burke - California Academy of Sciences

Evolution is the overarching theme for Voyages Through Time (VTT), a one-year integrated science high school curriculum consisting of six modules delivered on CD-ROM: Cosmic Evolution, Planetary Evolution, the Origin of Life, the Evolution of Life, Hominid Evolution, and the Evolution of Technology. VTT is being developed by SETI Institute, NASA Ames Research Center, California Academy of Sciences, and San Francisco State University, with funding from NSF and Combined Federated Charities, Foundation for Microbiology, Hewlett-Packard, NASA Astrobiology Institute, and NASA Fundamental Biology Program.

Major goals for VTT are for students to understand evolution as cumulative changes over time; the various processes underlying these changes; the differing time scales and rates of change; the connections and relationships across these realms of change; and science as a process for advancing our understanding of the natural world.

Evidence from extensive field testing indicates that the material and the CD-ROM approach are successful in classrooms. Teachers appreciate using technology to supplement traditional teaching. Scientists become welcome experts in the classroom via CD-ROM video clips. VTT PIs and consulting scientists have successfully faced many challenges in developing and revising the curriculum, and can share insights to help others developing a formal science curriculum. 
Magnetite crystals in ALH8400I carbonates: products of multiple origins

Kathie Thomas-Keprta $\bullet$ Lockheed Martin, Simon Clemett $\bullet$ Lockheed Martin, Christopher Romanek $\bullet$ Savannah River Ecology Laboratory, Dennis Bazylinski $\bullet$ Iowa State University, Joseph Kirschvink • California Institute of Technology, David McKay $\bullet$ NASA Johnson Space Center

McKay et al. ${ }^{1}$ proposed that primitive life existed on Mars approximately 4 billion years ago based on four lines of evidence, one which suggested that magnetite crystals in meteorite ALH84001 were formed by biogenic processes. Subsequently, Thomas-Keprta et al., have argued that up to $\sim 25 \%$ of these Martian magnetite crystals are magnetofossils, which on Earth are formed exclusively by terrestrial magnetotactic bacteria. In the case of the purported Martian biogenic population, we note that truncated hexa-octahedral magnetites on Earth are exclusively the product of biogenic activity - no natural or synthetic inorganic process is known to explain their presence in a terrestrial sample. The remainder of ALH84001 magnetites may be products of processes including precipitation from a fluid, thermal decomposition, or extracellular formation by dissimulatory Fe-reducing organisms. The origin of the majority of abiotic magnetites is not consistent with the thermal/shock decomposition process of $\mathrm{FeCO}_{3}$. We propose that the origins of magnetites in ALH84001 can best be explained as the product of multiple processes.

References

${ }^{1}$ McKay, D.S. et al. (1996). Science 286, 924-930.

2 Thomas-Keprta, K.L. et al. (2001). Proc. Nat. Acad. Sci. 98, 2164.

3 Thomas-Keprta, K.L. et al. (2000). GCA 64, 4049-4081.

\section{Insertional mutagenesis screen for genes involved in otic/vestibular development and function in xenopus tropicalis}

Marcela Torrejon $\bullet N A S A$ Ames Research Center, Erica Li $\bullet$ NASA Ames Research Center, Minh Nguyen $\bullet$ NASA Ames Research Center, Seth Winfree $\bullet$ NASA Ames Research Center, Esther Wang $\bullet$ NASA Ames Research Center, Sigrid Reinsch $\bullet$ NASA Ames Research Center Sensitivity to gravity is essential for spatial orientation. Consequently, the gravity receptor system is one of the phylogenetically oldest sensory systems, and the special adaptations that enhance sensitivity to gravity are highly conserved. The main goal of this project is to use Xenopus (frog) to identify genes expressed during vestibular and auditory development. These studies will lead to a better understanding of the molecular mechanisms involved in vestibular and auditory development and function.

We are using a gene-trap approach in Xenopus tropicalis with the green fluorescent protein (GFP) gene as the transgene reporter. GFP expression occurs only when the GFP gene is correctly integrated in actively transcribed genes. Using the GFP as a tag we can easily identify and clone the mutated gene. In addition, we can study the function of the mutated gene by analyzing the defects generated by insertion of the GFP transgene. To date we have tissue specific GFP expression in $X$. tropicalis including expression in ear, neural tube, kidney, muscle, eyes and nose. Our transgenic animals will soon reach maturity so that we can outcross them and analyze their progeny. Our next goal is to isolate RNA from our transgenics and clone the tagged genes using RACEPCR. Currently we are optimizing the RACE-PCR method using transgenics with crystallin GFP expression.

\section{Extrasolar planet color implies physical properties}

Wesley Traub • Harvard-Smithsonian Center for Astrophysics

The visible magnitude and color of an extrasolar planet can be used to infer the planet's albedo, diameter, temperature, mass, type (terrestrial or giant), presence or absence of an atmosphere, and possibly even the presence of chlorophyll. All of these properties can be estimated using standard color filters or very low-resolution spectral dispersion. The key is to use our experience with solar-system bodies, plus our ability to make modest extrapolations using models, and to assume that extrasolar planets can be classified as analogs of familiar solar-system objects.
After these properties are established, then somewhat higher-resolution spectroscopy can be used to search for atmospheric constituents including $\mathrm{H}_{2} \mathrm{O}, \mathrm{O}_{3}, \mathrm{O}_{2}, \mathrm{CH}_{4}$, and $\mathrm{CO}_{2}$.

\section{Convective plumes as "columns of life"}

Bryan Travis $\bullet$ Los Alamos National Laboratory, Nina Rosenberg $\bullet$ Lawrence Livermore National Laboratory, Jeffrey Cuzzi $\bullet$ NASA Ames Research Center

There is a great deal of evidence that water was abundant on Mars in the past and that much of that inventory still exists today in the sub-surface. Numerical simulations suggest that Martian groundwater will be subject to widespread hydrothermal convection driven by the geothermal gradient. Plumes are the dominant convective flow pattern for Mars conditions. Water temperatures within upwelling plumes can range from 40 to $90{ }^{\circ} \mathrm{C}$. These convective plumes are stable and long-lasting, and could provide a potential habitat for microbial life by bringing nutrients to and through microbial communities. Plumes may function as "columns of life." Further, for the higher geothermal heat fluxes of the past, flow will be episodic, characterized by occasional periods of enhanced flow rates and re-organization of the spatial arrangement of plumes, possibly providing a stimulus for evolution. Candidate microbial species would include anaerobic thermo-philes that utilize sulfur, carbon dioxide and iron, examples of which are found in abundance within mid-ocean ridge vent systems. Numerical simulation of convective plumes allows estimation of the maximum rate at which substrates and nutrients could be delivered to any microbial communities living therein. When coupled to microbial metabolic models, these simulations can provide an estimate of the size of such communities.

\section{Yellowstone National Park offers public access to astrobiology}

Catherine Tsairides $\bullet$ NASA Ames Research Center, Diane Chalfant $\bullet$ Yellowstone National Park, David Des Marais $\bullet$ NASA Ames Research Center, Jack Farmer • Arizona State University, Nancy Hinman • University of Montana, Rocco Mancinelli $\bullet$ SETI Institute, Lynn Rothschild $\bullet$ NASA Ames Research Center, David Ward $\bullet$ Montana State University, Linda Young $\bullet$ Yellowstone National Park

Yellowstone National Park (YNP) provides insights into the origin and evolution of life and the potential for life to exist beyond Earth. Biochemical clues hint that our early ancestors might have lived in hightemperature environments, including hot springs. Ancient spring alcoves on the flanks of Martian volcanoes invite comparisons with YNP, whose fossil-rich sinter spring deposits might, in turn, resemble deposits on Mars. A new visitor center is planned at Old Faithful, and interest in astrobiology is rising among the three million visitors of all ages that experience YNP each year. Thus the time has come to initiate a focussed outreach project that highlights both astrobiology and the work of the NASA Astrobiology Institute (NAI) in the Park. With support from the NAI outreach office, the Ames NAI Team, its partners, and YNP staff are creating ten trailside signs to exhibit astrobiology throughout YNP. This integrated team is also developing chapters on astrobiology and microbiology for the Yellowstone Resources and Issues Guide, a training manual for the interpretive research staff. Knowledge of Park resources and accurate information are fundamental elements of the interpretative equation. This team effort will help the general public to enhance their knowledge and skills and to access NASA research in astrobiology, including geology, microbiology, and space missions.

\section{The seX-files: searching for the origins of viral sex} Paul E. Turner $\bullet$ Yale University

If sex is defined as any exchange of genetic material between individuals, then sex is found to be extremely common in nature, and especially prevalent in microbes. But this is surprising given the many costs associated with sex, including the tendency for sex to separate welladapted combinations of genes. Therefore, evolutionary biologists have struggled for over a century to answer the question: why did sex evolve? One possibility is that sex is beneficial because it helps to combat the effects of harmful mutations. For instance, sex can allow two parents to 
recombine their genetic material and produce offspring that contain fewer harmful mutations. Another possibility is that sex is beneficial because it allows faster evolution when individuals encounter novel environments. An asexual lineage adapts by adding only a single favorable mutation to the genome at a time. In contrast, sex can combine favorable mutations from multiple lineages at once, and thus speed up the rate of adaptation.

Many medically and agriculturally important pathogens are viruses that contain RNA as their genetic material; examples include HIV, poliovirus, and influenza. RNA organisms differ from their DNA brethren because RNA molecules are not proofread to correct the mistakes that occur during gene replication. For this reason, RNA viruses feature extremely high rates of genetic mutation, and most of these mutations are deleterious. Thus, RNA viruses may have evolved sex because it facilitates removal of these harmful mutations from their genomes. In addition, RNA viruses continually induce evolutionary responses in their hosts (or host immune systems), and are thus often exposed to novel or changing environments. Therefore, RNA viruses may have evolved sex because it generates the genetic variation needed to address the challenge of adaptation in variable environments.

Laboratory experiments with viruses offer very powerful systems to examine the evolutionary origins of sex. RNA viruses are particularly instructive because they feature a sexual mechanism that is not found anywhere else in nature. Sex in most organisms occurs through some form of recombination (joining of gene fragments). In contrast, only certain RNA viruses feature sex via the process of reassortment. Here, sex is achieved by splitting the RNA genome into several smaller segments and hybrid offspring are produced by randomly reassorting the segments. An analogy is the shuffling of two hands of playing cards to create a series of novel hands. Experimental data suggest that reassortment evolved to combat the buildup of harmful mutations, because this process is instrumental in purging deleterious mutations from RNA viruses. ${ }^{1}$ However, results do not support the idea that reassortment evolved because it provides beneficial variation in novel environments. Rather, these data indicate that too much sex can be a bad thing; abundant sex allows promiscuous viruses to selfishly ensure that their inferior genes reach subsequent generations. ${ }^{2}$

References

${ }^{1}$ Chao, Tran \& Tran (1997). The advantage of sex in RNA virus phi 6. Genetics 147, 953-959.

2 Turner \& Chao. (1999). Prisoner's dilemma in an RNA virus. Nature 398, 441-443.

\footnotetext{
The pressure factor in Europa's aqueous evolution Steven Vance $\bullet$ University of Washington, J. Michael Brown • University of Washington, Jeffrey Kargel $\bullet$ U.S. Geological Survey Low-pressure, low-temperature thermodynamic modeling software (FREZCHEM) has been used to simulate the evolution of Europa and other planetary bodies. While investigators refer to experimental results in consideration of the likely effects of high pressures on this evolution, calculations have not yet taken them into account. Application of highpressure equations of state is needed to track Europa's chemical evolution, from addition of gases to the ocean, metamorphism of hydrated salts on the seafloor, and temperature oscillations of the system in response to changes in tidal heating. Incorporating pressure behavior into the models will direct further experiments needed to improve the model and thus our understanding. The formation of Europa and the composition of its ocean can be probed by experimental and computational means. These efforts also have applications to the modeling of other planetary bodies and of Earth's deep ocean. The chemical potentials for aqueous solutions can be derived from the speed of sound measured as a function of pressure and temperature. These parameters are gathered in our laboratory through the method of impulsive stimulated scattering. Thus far, an equation of state has been determined for aqueous sodium sulfate up to $34 \mathrm{kbar}$ and $573 \mathrm{~K}$. Others have determined the phase equilibria for sodium and magnesium sulfatehydrates up to 4 kbar.
}

Biological potential of Martian hydrothermal systems Stacy Varnes • University of Colorado, Bruce Jakosky • University of Colorado

Although a global average of energy available to potentially support life on Mars has been estimated, issues of how the energy is distributed and which environments have the greatest potential to support life remain unresolved. To address these questions we have developed numerical geochemical models of Martian hydrothermal systems. For each model, the composition of the groundwater and the host rock with which it interacts must be defined. Host rock compositions are constrained by the composition of SNC meteorites, and are reacted at high temperature with a series of waters. Resulting vent fluids are reacted with increments of fresh groundwater, simulating the mixing that occurs in hydrothermal systems. Groundwaters are either pure water in equilibrium with the Martian atmosphere or with Pathfinder-composition soils and the atmosphere. For each model run, the Gibbs free energy of reactions that are important for terrestrial chemosynthetic organisms and likely to be representative for putative Martians are calculated. Results indicate that substantial amounts of energy may be derived from these reactions, depending upon the oxidation state of the groundwater, type of host rock, and whether saturated species precipitate to equilibrium. Thus, it is unknown whether sufficient energy is available to support Martian life, although it is likely that suitable environments exist.

\section{Integrin-mediated cellular response to hypergravity} $W$. Vercoutere $\bullet N A S A$ Ames Research Center, C. Roden $\bullet N A S A$ Ames Research Center, E. Holton $\bullet$ NASA Ames Research Center, $N$. Searby $\bullet$ NASA Ames Research Center, R. Globus $\bullet$ NASA Ames Research Center; University of California, San Francisco, E. Almeida $\bullet$ NASA Ames Research Center; University of California, San Francisco Life on Earth has evolved under the constant influence of gravity (1-g). Exploration and development of space, however, means that life is being exposed to microgravity and may in the future be exposed to planetary hypergravity. Living organisms in gravity environments deviating from 1-g show significant physiological alterations, however the corresponding cellular responses are still poorly understood. We hypothesize that cellular responses to gravity are in part influenced by specific cellular signaling pathways dependent on integrin adhesion to the extracellular matrix. Earlier results from our laboratory show that centrifugation increases fibronectin matrix survival signaling via integrins in primary osteoblasts and fibroblasts. This and other experimental evidence suggests that the cellular responses to different gravity levels fall along a continuum, so that hypergravity and normal gravity experimental results can be used to estimate the responses that may result from a microgravity environment. In this study we investigate responses of cultured primary osteoblasts to hypergravity conditions using a custom 1-ft-diameter vented Eppendorf centrifuge (model 5804) placed inside an environmental cell culture chamber that provides heating, cooling, humidity and $\mathrm{CO}_{2}$ control. Our preliminary results indicate that a 50 -g hypergravity stimulus for 24 hours doubled the proliferation of primary osteoblasts compared with the 1-g control. To test the effect of hypergravity on matrix adhesion, focal adhesion kinase (a kinase involved in matrix adhesion signaling and cell survival) was fused to green fluorescent protein (GFP-FAK), and expressed in primary osteoblasts that were then centrifuged at 50 -g for 24 hours. The number and area of FAK complexes increased dramatically in 50-g compared with the 1-g control. Together these results suggest that increased matrix signaling in hypergravity conditions upregulate cell survival and proliferation pathways. Current studies in our laboratory are focusing on the osteoblast proteome response to hypergravity. (Supported by NASA: 00-OBPR- 01-066.)

\section{Gravity level modulates mRNA expression of metabolic enzymes in the liver}

C.E. Wade $\bullet$ NASA Ames Research Center; University of Medicine and Dentistry of New Jersey, L.A. Baer $\bullet$ NASA Ames Research Center; University of Medicine and Dentistry of New Jersey, T.P. Stein - NASA Ames Research Center; University of Medicine and Dentistry of New Jersey 
Life beyond the planet of origin encompasses exposure of living systems to variations in gravity levels. In the present studies we have focused on the effect of variable gravity levels on the metabolism of vertebrates by studying liver tissue changes in mRNA expression and concentrations of enzymes. Rats were exposed to chronic (14-21 day) increases in gravity by centrifugation, or simulated micro-gravity using the hind limb suspension model. Expression of mRNA was determined by using the Affymetrix gene chip with the metabolic pathways of interest identified a priori. With hind limb suspension there was a significant increase in mRNA expression of enzymes involved in gluconeogenesis $(+39 \%)$ and a reduction with hyper-gravity $(-32 \%)$. Amino acid transferase activity was increased $(+34 \%)$ during suspension and decreased with hyper-gravity $(-16 \%)$. For amino transferases involved in gluconeogenesis there was an increase $(+68 \%)$ with hind limb suspension and a decrease during hyper-gravity $(-26 \%)$. With hind limb suspension there is a preferential shift to glucose utilization in muscles, necessitating an increase in liver glucose production via metabolism of amino acids. This shift in metabolism is similar to that observed during space flight. During exposure to hyper-gravity the opposite occurs. We suggest htat there are shifts in liver substrate metabolism associated with gravity that form a continuum across the level of exposure.

\section{Life in rock: a molecular analysis of endolithic} microbial communities in the rocky mountain region Jeffrey Walker $\bullet$ University of Colorado, Norman Pace • University of Colorado

The endolith, comprised of the pore-spaces in rock, is a ubiquitous habitat for microbes on Earth, and may harbor life elsewhere in the Universe. Endolithic communities exist in the most extreme environments on Earth, such as the Ross Desert in Antarctica. The endolithic environment provides microorganisms with a growth surface, shelter from harmful radiation, mineral nutrients, and moisture. Analogous endolithic environments must occur on Mars today, and may contain evidence of past or present life. The biological and physical dynamics of endolithic communities on Earth inform the search for life on other bodies in the solar system. Despite a global distribution, little is known about these ecosystems on Earth.

This study examines the composition and structure of microbial communities that inhabit a variety of rock types in the Rocky Mountains of North America. Phylogenetic analysis of ribosomal RNA genes cloned from communities inhabiting sandstone, limestone, and granite suggests that rock type is a significant determinant of community composition. Comparing results from different communities, including communities from the Ross Desert in Antarctica, shows that phylogenetically related, but unique microbes form similar kinds of communities in different types of rock.

\section{Neutron computed tomography reconstructions of microbial structures in carbonate and silicate rocks M.C. Wilding • University of California, Davis, D.Y. Sumner $\bullet$ University of California, Davis, C.E. Lesher $\bullet$ University of California, Davis, K.E. Shields $\bullet$ McClellan Nuclear Radiation Center, D. Reap $\bullet$ McClellan Nuclear Radiation Center, W.J. Richards $\bullet$ McClellan Nuclear Radiation Center}

Neutron computed tomography (CT) is a relatively new technique that shows great promise for the non-destructive imaging of organic materials in rocks. Neutron CT, which is analogous to X-ray CT, uses computer algorithms to reconstruct the three-dimensional structure of a sample from a series of $\mathrm{N}$ radiographs. The strength of $\mathrm{N}-\mathrm{CT}$ for imaging biological structures comes from its sensitivity to light elements, especially hydrogen and carbon. Thus, it is a technique that will enhance studies of endolithic and fossil microbial communities, particularly in conjunction with X-ray CT, which is sensitive to heavy elements. N-CT techniques developed at the UC Davis McClellan Nuclear Radiation Center are being refined to obtain the maximum contrast and spatial resolution for organic materials in rock. Transmission-intensity tables for water, carbonate, silicates, and organics demonstrate that their scattering profiles are sufficiently different to allow high-resolution imaging of the distribution of hydrogen and carbon in diverse rock types. Results to date include detailed images of: (1) organic and fluid inclusions in neoarchean calcitic microbialites; (2) modern endolithic mircobial communities in calcite; (3) the distribution of water-wetted surfaces in sand; and (4) various experimental combinations of carbonate, organic carbon, and graphite.

\section{Peptides at membrane surfaces and their role in prebiotic evolution}

Michael Wilson • University of California, San Francisco, Andrew Pohorille $\bullet$ NASA Ames Research Center

Protocells had to transport ions and organic matter across membranous walls separating the cell interior from the environment, capture and utilize energy and transduce environmental signals. In modern organisms, these functions are carried out by membrane proteins. We make the parsimonious assumption that in the protobiological milieu the same functions were carried out by peptides.

In a series of detailed, molecular-level computer simulations we demonstrated how these peptides in contact with membranes can acquire ordered structures and functions. We have investigated the stability of monomers and dimers of the peptide (LSLLLSL) 3 in a membranemimetic system. Both transmembrane and parallel, in-plane orientations of the monomer are stable, with the parallel orientation being the more stable state. A tetrameric bundle of this peptide is capable of transporting protons across membranes.

We have also investigated the transmembrane pore of the influenza M2 protein. This aggregate of four identical alpha-helices, each built of 25 amino acids, forms an efficient and selective voltage-gated proton channel. Our simulations explain the gating mechanism, which can involve strands of hydrogen-bonded water through the pore or proton transfer through tautomerization of protein residues. The channel can be re-engineered to act as a simple proton pump.

\section{Novel viruses from extreme thermal environments} Mark Young • Thermal Biology Institute, George Rice • Thermal Biology Institute, Kenneth Stedman • Thermal Biology Institute, Jamie Snyder • Thermal Biology Institute, Blake Wiedenheft $\bullet$ Thermal Biology Institute, Timothy McDermott $\bullet$ Thermal Biology Institute Viruses of extreme thermophiles are of great interest because they serve as model systems for understanding the biochemistry and molecular biology required for life at high temperatures. In this work, we report the discovery, isolation and preliminary characterization of novel viruses and virus-like particles from extreme thermal acidic environments $\left(70-92{ }^{\circ} \mathrm{C}, \mathrm{pH} 1.0-4.5\right)$ found in Yellowstone National Park, USA. This is the first report of viruses and virus-like particles isolated from hightemperature, acidic environments present in Yellowstone National Park. Six unique particle morphologies were found in Sulfolobus enrichment cultures. Three of the particle morphologies are similar to viruses previously isolated from Sulfolobus species from Iceland and/or Japan. Sequence analysis of their viral genomes suggests that they are related to the Icelandic and Japanese isolates. In addition, three new virus particle morphologies that had not been previously observed from thermal environments were found. These viruses appear to be completely novel in nature.

\section{Impacts and the habitability of the Hadean Earth Kevin Zahnle $\bullet$ NASA Ames Research Center, Norman H. Sleep $\bullet$} Stanford University

The Hadean is usually defined as the eon on Earth before the rock record, a definition neither useful nor generally applicable. A different definition - the Earth before life-is at least consistent with the paleontological foundation of the geological time scale, but again it is difficult to apply in practice (some might place the Hadean as late as $2.7 \mathrm{Ga}$ !) and inapplicable or irrelevant to other worlds. A third choice would be to focus on the time before plate tectonics, a time that is poorly understood and hard to specify. The most useful choice is to define the Hadean as the time when impacts ruled the Earth. Such a definition is applicable to other worlds, and it focuses on a fundamental change in 
planetary evolution. For decades now it has been obvious that the coincidence between the timing of the end of the lunar late bombardment and the appearance of a rock record on Earth is probably not just a coincidence. While the Moon was struck by tens of basin-forming impactors ( $\sim 100 \mathrm{~km}$ objects making $\sim 1000 \mathrm{~km}$ craters), the Earth was struck by hundreds of similar objects, and by tens of objects much larger still. The largest impacts on Earth would have been big enough to evaporate the oceans, and the ejecta massive enough to envelop the Earth in $100 \mathrm{~m}$ of rock vapor. Although these largest impacts would have been very rare - perhaps two or three in total - they would have been important to evolution, as only the most protected subsurface ecological niches could have served as refugia. A more exciting biotic refuge would be in impact ejecta exchanged between planets. At early times traffic between Earth and Mars (or even Venus) would have been frequent enough to speculate that the worlds were biologically linked. Smaller impacts were also more frequent. On average, a Chicxulub fell every 100000 years. When one imagines the Hadean one imagines it with craters and volcanoes: crater oceans and crater lakes, a scene of mountain rings and island arcs and red lava falling into a steaming sea under an ash-laden sky. If toned down a bit - not all the volcanoes erupting simultaneously - much of this picture makes good sense. The oceans would have been filled with typically hundreds of meters of weathered ejecta, most of which were ultimately subducted but taking with them whatever they reacted with at the time $-\mathrm{CO}_{2}$ was especially vulnerable to this sort of scavenging. The climate, under a faint sun and with little $\mathrm{CO}_{2}$ to warm it, may have been in the median extremely cold, the oceans under ice, with on occasion the cold broken by brief (10s to 1000 s of years) episodes of extreme heat and steam following the larger impacts. Ancient zircons indicate that liquid water was at times present at the surface at times of weathering. We have found no abiotic buffer that might maintain the Hadean climate at around $100^{\circ} \mathrm{C}$, although there are buffers that can maintain the climate at $200{ }^{\circ} \mathrm{C}$ (hot mantle inhibiting carbonate subduction, causing Earth's $\mathrm{CO}_{2}$ inventory to build up in the atmosphere) or at less than $0{ }^{\circ} \mathrm{C}$. Biogenic greenhouse gases, in particular methane, can presumably be ratcheted to maintain any climate that the biota can survive. In summary, the age of impacts seems sufficiently unlike the more familiar Archean that came after that it seems useful to give this time its own name, a name we already have, and that, if applied to the Hadean that I have described, actually has some geological value.

\section{Astrobiology missions}

\section{The genetics of extraterrestrial life?}

\section{Steven Benner $\bullet$ University of Florida}

A central question of Astrobiology as it relates to NASA missions asks: "How would we recognize life if we were to find it?" The elegant simplicity of the Watson-Crick model for DNA provides only the illusion of universality. Ultimately, we must ask: "Is DNA the only structure capable of supporting genetics?" Here, we address this question using synthetic organic and physical chemistry. This talk will focus on alternative genetic systems that have been prepared and studied in the laboratory. These include DNA-like systems that have 12 "letters" instead of the four found in naturally occurring DNA, functionalized DNA systems that may support enhanced catalysts, and backbonemodified DNA analogs. We have developed general rules that guide and predict the behavior of non-standard genetic systems. From these, we have developed prescriptions for how to search for "biosignatures," molecular structures likely to be universal in life, found wherever life exists, and unique to life, generated by no non-living process. Hydrogen bonding must be considered in any "second-generation" model to predict, explain, and manipulate the behavior of nucleic acid-like artificial genetics systems in water. A polyanionic character also appears to be necessary, given chemical constraints, in water. Lastly, we provide guidelines for designing artificial genetic systems.

\section{Quantitative approaches to evaluating the biogenecity of fossil stromatolites}

Per Jögi • University of California, Los Angeles, Bruce Runnegar • University of California, Los Angeles

A perennial problem for life detection in ancient and/or extraterrestrial sedimentary sequences is evaluating the biogenicity of complex bedforms known as stromatolites. Strictly speaking, all stromatolitic structures should be regarded as abiotic until there is sufficient evidence to identify them as microbialitic (microbialites). In order to improve this approach to life detection, we are developing quantitative methods to understand the evolution of complex surfaces in $2+1$ dimensions. This work follows a procedure introduced by Grotzinger and Rothman (Nature 383, 423-425, 1996) but arrives at somewhat different conclusions. Our synthetic analysis suggests that the power-law scaling exponent $(2.01 \pm 0.03)$ and fractal dimension $(2.50 \pm 0.02)$, which were obtained from frequency spectra of natural and artificial sections of Palaeoproterozoic stromatolites are not diagnostic of processes governed by the famous KPZ (Kardar-Parisi-Zhang) equation. Instead, KPZ growth has a scaling exponent of approximately 1.8, a difference which we consider significant. Thus, statistics obtained from frequency spectra represent a blunt tool when attempting to assess biogenicity.

\section{The Artemis Mars Multi-Scout Mission}

David Paige $\bullet$ University of California, Los Angeles

The Artemis Mars Multi-Scout Mission is a new mission concept that is currently being studied for NASA's Mars Scouts Program. Its major goals are to recover the science objectives of the Mars Polar Lander, and explore multiple landing sites on the Martian surface of high scientific interest at a wide range of latitudes, making measurements which will significantly improve our understanding of the behavior and distribution of water on Mars, the Martian climate, geology, and potential for life.

In the present concept, the Artemis carrier vehicle will be launched to Mars in September 2007 on a Type 2 trajectory, and propulsively inserted into an elliptical polar orbit. During the course of its two-year mission, the orbiter will release three identical small landers, which will be precisely targeted to a diverse range of scientifically interesting landing sites such as the polar layered deposits, and newly discovered layered sedimentary deposits at lower latitudes. The design of the Artemis landers will be patterned after the Mars Express Beagle 2 lander, which will employ an aeroshell and air bag landing system. The Artemis orbiter will serve as a communications link for the Artemis landers during their three-month surface missions. The flexibility inherent in the Artemis mission design concept can allow for many variations, including more landers, and orbiting instruments.

\section{Potential of fluid inclusion studies in astrobiological exploration \\ John Parnell $\bullet$ University of Aberdeen}

Fluid inclusion studies have the potential to help us understand the temperature record of impact crater sites, their history of fluid circulation, the preservation of organic matter, and the subsequent behaviour of exposed rock surfaces. All of these aspects are valuable constraints on the potential of impact crater rocks to hold a record of life.

Studies of terrestrial impact craters show that hydrothermal circulation associated with impact involved fluids at $100-400{ }^{\circ} \mathrm{C}$. However gradual cooling of a hydrothermal system would allow lower-temperature subsurface biotas to colonize impact fracture systems. Falling temperatures also cause mineral precipitation, preserving a record of the habitat. The inclusion record of lower-temperature inclusions in cross-cutting trails shows that impact fracture systems are reactivated by later fluids, which would introduce fresh nutrients and allow continued or renewed microbial activity.

Impacts should destroy pre-existing fluid inclusions, but older inclusions survive outside terrestrial craters. Thus blocks ejected by impact may record a succession of hydrothermal events. The release of 
inclusion fluids by crushing could preserve any complex biomolecules representing ambient life during mineral precipitation. In situ analysis
(FLIRT: Fluid Inclusion Release Test) has the potential to record a biosignature of life on Mars.

\section{Technology in support of astrobiology science}

\begin{abstract}
Atmospheric biosampler for planetary use
David Alburty $\bullet$ Midwest Research Institute, Robert Barton $\bullet$ Midwest Research Institute, Robert Huebner $\bullet$ Midwest Research Institute, Kelly L. Brown $\bullet$ Midwest Research Institute

Midwest Research Institute (MRI) has developed a technology (SpinCon c) for sample collection and concentration proven to be ideally suited for a broad range of advanced air sampling requirements, including the collection of bioaerosols, particulate matter, and soluble vapors. These include micron-sized particles, airborne biological particulates, and semi-volatile and non-volatile compounds.

MRI is conducting this proof-of-concept development and testing of a system for collecting and analyzing a very large volume of extraterrestrial planetary atmospheres (initially Martian and Titanic) for the presence of signs of panspermic or other life; specifically, nucleotides and proteins. MRI plans to design and build a custom proof-of-concept device and operate it in an environmental chamber at MRI or NASA.

The SpinCon relies on collection and concentration in a tangential slit-inlet cyclonic liquid contractor. Mechanisms responsible for sample collection and concentration, system construction, and performance requirements will be discussed. Potential liquids for sampling on Mars and Titan, and biodetection using sampler cleanup and SYBR Green I nucleic acid stain (Molecular Probes, Inc.) and a miniaturized flouromoter will also be discussed.
\end{abstract}

\section{Environmental regulation of microbial community structure \\ L. Bebout $\bullet$ NASA Ames Research Center, B. Bebout $\bullet$ NASA Ames Research Center, D. Des Marais $\bullet$ NASA Ames Research Center, G. Heyenga $\bullet$ University of Colorado, F. Nelson $\bullet$ Foothill-DeAnza Internship Program}

Most naturally occurring microbes live in complex microbial communities consisting of thousands of phylotypes of microorganisms living in close proximity. Each of these draws nutrients from the environment and releases metabolic waste products, which may in turn serve as substrates for other microbial groups. Gross environmental changes, such as irradiance level, hydrodynamic flow regime, temperature or water chemistry, can directly affect the productivity of some community members, which in turn will affect other dependent microbial populations and rate processes. As a first step towards the development of "standard" natural communities of microorganisms for a variety of potential NASA applications, we are measuring biogeochemical cycling in communities of microorganisms that are maintained in our laboratory, but were created using natural microbial mat communities as inocula. The responses of these laboratory communities to controlled shifts in incubation conditions are being determined.

This research requires close linking of environmental monitoring, with community composition in a closed and controlled incubation setting. We are developing new incubation chamber designs to allow for this integrated approach to examine the interplay between environmental conditions, microbial community composition and biogeochemical processes.

\section{From field to laboratory: a new database approach for linking microbial field ecology with laboratory studies \\ L. Bebout $\bullet$ NASA Ames Research Center, R. Keller $\bullet$ NASA Ames Research Center, S. Miller $\bullet$ North Carolina State University, $L$. Jahnke $\bullet$ NASA Ames Research Center}

The Ames Exobiology Culture Collection Database (AECC-DB) has been collaboratively developed by microbial ecologists and information technology specialists. It provides for detailed webbased archiving of field sample information to document microbial habitation of specific ecosystem micro-environments. Documentation and archiving continues as pure cultures are isolated, metabolic properties determined, and DNA extracted and sequenced. In this way metabolic properties and molecular sequences are clearly linked back to specific isolates and the location of those microbes in the ecosystem of origin.

Use of this database system presents a significant advancement over traditional book-keeping wherein there is often little or no information regarding environments from which microorganisms were isolated. Generally there is only an overall ecosystem designation (i.e. hotspring). However within each ecosystem there are a myriad of microenvironments with very different properties. Determining exactly which micro-environment a given microbe comes from is critical in designing appropriate isolation media and interpreting physiological and ecological properties.

We are currently using the database to aid in the isolation of a large number of cyanobacterial species and will present results by PI's and students demonstrating the utility of this new approach.

\section{An electrospray-ionization ion mobility/cylindrical mass spectrometer for in situ detection of organic} compounds on Mars, Europa and Titian

Luther Beegle $\bullet$ Jet Propulsion Laboratory, Isik Kanik $\bullet$ Jet Propulsion Laboratory, Paul Johnson $\bullet$ Jet Propulson Laboratory

Development of a miniature, low-power, and low-mass detector system, which eliminates the problems associated with the conventional detection techniques (fragmentation, inadequate sensitivity, high vacuum) is a requirement for in situ exobiological analysis on planetary bodies such as Mars, Europa and Titan. The potential of high-resolution electrospray ionization/ion mobility spectrometry (ESI/IMS) as an analytical separation tool in analyzing liquid samples to search for the chemical signatures of life is demonstrated. (Beegle L.W. et al. (2001), Beegle L.W. et al. (2002)). This includes the attachment of a small cylindrical ion trap (CIT) to the end of the IMS cell.

ESI is a powerful ionization method capable of ionizing large organic molecules without fragmentation. IMS operation is analogous to that of time-of-flight (TOF) spectrometers except that it operates at high pressures. The mobility itself is related to the size and shape of the ion imparting a second dimension which enables IMS to separate isomers. IMS has been demonstrated to be very sensitive in detecting organic compounds, and by attaching a CIT to the end of the IMS we are able to uniquely identify organic molecules. The ultra high-resolution ESIIMS-CIT technique, has a great potential to fulfill the NASA requirement for detecting and accurately analyzing a mixture organic compounds.

\section{Development of microarray assays for biomarker identification in astrobiology}

Carlos Briones $\bullet$ Centro de Astrobiologia, Victor Parro $\bullet$ Centro de Astrobiologia, Juan Perez-Mercader $\bullet$ Centro de Astrobiologia

Techniques for biomarker detection (nucleic acids, proteins, lipids or metabolites) have been extensively developed. Microarray technology allows attaching thousands of probes within a few square centimetres on a solid support. Smaller reaction volumes and higher reaction kinetics, together with their great potential for miniaturization and robotization, make microarray technology an ideal tool for in situ analysis of biomarkers in astrobiology.

Our first approach consists in the covalent attachment of a set of known antibodies to a glass slide to make an array pattern of spots. Antigens, either purified or in complex mixtures, are fluorescently labelled and incubated in the array. Excitation with the appropriate laser light shows bright spots where the antigen has been bound. We are testing several parameters involved in this process, including: antibody 
stability, labelling/incubation/washing buffers and times, different fluorochromes, labelling efficiencies for the antigen in complex mixtures (e.g. soil extracts), etc.

Our results show that it feasible to generate, produce and analyze good quality protein microarrays through simplified protocols suitable for in situ analysis. We have simplified several steps so that it is possible to label and incubate in the same buffer without further purification. This is crucial in order to achieve full automation of the system.

\section{A compact high-resolution 3-D imaging spectrometer for discovering oases on Mars}

Jian Ge • Pennsylvania State University, Jonathan Lunine $\bullet$ University of Arizona, Robert Brown $\bullet$ University of Arizona, Laurence Soderblom - United States Geological Survey, Roger Yelle $\bullet$ University of Arizona Two key infrared instrument components, high-resolution silicon prisms and cryogenic image slicers, are being developed at PSU under NASA support for potential applications in future Mars missions. These new instrument components are planned to be used for detecting and characterizing possible organic compounds on the Martian surface at infrared (IR) wavelengths. It is a compact, robust and light-weight 3D near-IR imaging spectrometer, enabling an order of magnitude improvement in spectral resolution and observing efficiency and also large simultaneous wavelength coverage. Silicon prisms provide at least 3 times higher spectral dispersion than any commercially made prisms, which will be the key elements for making the instrument compact enough and simultaneously provide high spectral resolution to resolve the weak spectral features from organic materials. The reflective imaging slicers enable us to collect spectral information from the Mars surface in 3-D form - two spatial dimensions and one spectral dimension. This unique capability obviates the need to make many scans to build up the data cube as in traditional instruments, resulting in an order of magnitude increase in observing efficiency. We have successfully developed high-quality silicon prisms suitable for space missions. The optics for image slicers has been designed and procured. Progress will be reported.

\section{Gretel: A Linux computer cluster for bioinformatics} Richard Graul $\bullet$ ISLE, Karl Schweighofer • Carnegie Mellon

Parallel processing refers to the concept of speeding up the execution of a program by dividing the program into multiple fragments that can execute simultaneously, each on its own processor. A program being executed across $n$ processors may execute $n$ times faster than it would using a single processor.

Dealing with large databases of sequence information is an evergrowing challenge in bioinformatics. Fortunately, the common task of similarity searching is readily amenable to parallel processing. By partitioning either the query or target databases, one can divvy up the search space amongst multiple processors. Therefore, clusters of networked computers have emerged as an effective and relatively inexpensive approach to parallel processing in bioinformatics.

The NASA Center for Astrobioinformatics was recently established at Ames as part of the effort to integrate biotechnology at NASA. The Center brought its computer facility on-line in October 2001. At the heart of the facility is a Linux-based computer cluster called Gretel. Gretel consists of ten dual Pentium computers networked for parallel processing. The system is used primarily for bioinformatics research activities. The focus of our research is gene structure and function. Currently implemented are several bioinformatics tools, including BLAST and Pfam. Please visit our web site: http:// genomics.arc.nasa.gov/.

\section{A high-pressure ionization source for in situ ionization and detection of organic molecules in the ambient Martian atomsphere}

Isik Kanik • Jet Propulsion Laboratory, Luther Beegle • Jet Propulsion Laboratory, Paul Johnson $\bullet$ Jet Propulsion Laboratory

We have designed, constructed and characterized a new high-pressure (1-5 Torr) hollow cathode discharge source (HCDS) that can be utilized as an ionizer in a wide variety of mass analyzers. It is able to function under ambient Martian atmospheric conditions (Temp and Pres). The ionizer produces a stream of $\left(\mathrm{H}_{2} \mathrm{O}\right) \mathrm{H}^{+}$ions from pure water vapor introduced into the ionizer from a small liquid water container. These ions react with air-borne samples and ionize organic species present via proton-transfer-reaction (PTR) without ionizing the Martian atmospheric components. PTR is a better choice for ionization over other conventional ionization techniques (i.e. $\mathrm{Ni}_{63}$ source, electron impact, etc.) for in situ detection of large organic molecules on Mars for two reasons: (a) it utilizes a soft ionization technique so large organic species will not fragment, and (b) the residual Martian atmosphere and most non-organic species present on the surface will not react with $\mathrm{H}_{3} \mathrm{O}^{+}$as they have lower proton affinities than $\mathrm{H}_{2} \mathrm{O}$ (Lindinger W. et al. (1998)).

To facilitate the ionization of target molecules through the PTR process, a beam of $\left(\mathrm{H}_{2} \mathrm{O}\right) \mathrm{H}^{+}$ions, generated by the $\mathrm{HCS}$, are extracted into a reaction chamber which ionizes target species and then into a mass spectrometer. This ionizer can be used with variety of mass spectrometers (QMS, ion trap, ion mobility etc.).

\section{Microbial etching textures of olivine: use as a biosignature?}

Teresa G. Longazo $\bullet$ Hernandez Engineering, Susan J. Wentworth $\bullet$ Lockheed Martin, Simon J. Clemett $\bullet$ Lockheed Martin, Gordon Southam $\bullet$ University of Western Ontario, David S. McKay $\bullet$ NASA Johnson Space Center

We are investigating the effect microbial etching has on olivine surfaces. The goal is to determine whether such features could serve as a biosignature. Three series of experimental trials have been conducted using both biotic and abiotic conditions to characterize the morphological changes on olivine surfaces. The most pervasive change observed is the preferential surface etching/dissolution features, which occur as either pits or channels aligned along the olivine (001) crystallographic axis. ${ }^{1,2,3}$ Microbial colonies appear to preferentially align themselves along the edges of the larger etch structures. The presence of nanometersized imprints (nanoetching), which mimic the outline of the microorganisms and their biofilms, are observed on these same edges. Such features are absent in the abiotic experiments, suggesting their presence is uniquely associated with the microorganisms. However, qualitative morphology alone is not a robust biosignature. We suggest that, with further statistical analyses, these nanoetching features could serve as a potential biosignature for microbial activity. Future work will investigate the vertical profiles of the etch/dissolution surfaces using white light scanning interferometry.

References

${ }^{1}$ Longazo, T.G. et al. (2001). LPSC XXXII.

${ }^{2}$ Longazo, T.G. et al. (2002). LPSC XXXIII.

${ }^{3}$ Welch, S.A. \& Banfield, J.F. (2002). Geochim. Cosmochim. Acta, 66, 213-217.

\section{Conserved gene clusters in magnetotactic bacteria} Cody Nash $\bullet$ California Institute of Technology

The understanding of the biomineralization processes in magnetotactic bacteria could facilitate numerous biotechnological applications and aid the identification of magnetic fossils.

The mechanisms by which magnetotactic bacteria synthesize intracellular chains of membrane-bound magnetite crystals (magnetosomes) are poorly understood. The synthesis of single-domain magnetite crystals by numerous species raises the possibility that similar, conserved genes may control these biomineralization processes. This hypothesis is supported by the recent finding that two genes encoding magnetosomal proteins were found in a cluster of genes (the Mam cluster) conserved between three magnetotactic species. A reciprocal BLAST comparison of the draft genomes of Magnetospirillum magnetotacticum (MS-1) and Magnetococcus sp. (MC-1) revealed 11 clusters of conserved genes. In four of these clusters the order was more than $75 \%$ conserved between the two species. One of these clusters in which order was conserved was the documented Mam cluster, which acted as a positive control for this study. The other three clusters in which order is conserved include genes that models predict to be associated with chemotaxis, ion transport, and metabolism. 


\section{The Space Station biological research project: an artificial gravity laboratory}

Jon Rask • Lockheed Martin Space Operations, Melissa Kirven-Brooks - Lockheed Martin Space Operations, Francis Donovan • Lockheed Martin Space Operations, Carol Elland $\bullet$ Lockheed Martin Space Operations, Patricia Larenas $\bullet$ Lockheed Martin Space Operations, Sharmila Bhattacharya $\bullet$ Lockheed Martin Space Operations, Gabe Meeker $\bullet$ Lockheed Martin Space Operations; NASA Ames Research Center, Orlando Santos $\bullet$ Lockheed Martin Space Operations

The environment aboard the International Space Station (ISS) provides an extraordinary opportunity to study biology in reduced gravity. While the amount of data from experimentation on biospecimens in microgravity and hypergravity continues to grow, opportunities for long-duration science have been unavailable, and our understanding of the biological response to partial or intermittent gravity remains very limited.

Ground-based models provide simulations, however, we can only fully investigate the problem with a laboratory in free-fall around the Earth. The Project will provide the research community with technology designed to vary the amount of g-force (0.01-2.00 g) applied on biological specimens. Multi-generational genetic and developmental biology studies in simulated Lunar, Martian, or Europa gravity are possible in a $2.5 \mathrm{~m}$ Centrifuge Rotor that will be installed in the Centrifuge Accommodation Module of the ISS.

Habitats including a Cell Culture Unit, an Advanced Animal Habitat with Animal Enclosure Modules, an Insect Habitat, and a general purpose Incubator will support model organisms like $A$. thaliana, $C$. elegans, S. cervisiae, D. melanogaster, and rodents in microgravity or partial gravity exposure with $1 \mathrm{~g}$ controls. The Life Sciences Glovebox allows for access, manipulation, and preparation of specimens inside a contained work volume

\section{Community-level physiological and molecular profiling of microbial communities}

Michael S. Roberts $\bullet$ Dynamac Corporation, Jay L. Garland • Dynamac Corporation, Lanfang H. Levine $\bullet$ Dynamac Corporation, Aaron L. Mills • University of Virginia

Robust measures of microbial community richness and functional diversity have been hindered by technological and analytical limitations.

The rapid assessment of sole carbon source utilization by whole communities (i.e. community-level physiological profiling or CLPP) has proven to be an effective tool for evaluating microbial community dynamics, but not for characterizing relative rates of carbon source use. Selective enrichment during community profiling is often unavoidable due to requirements for long incubation periods (i.e. 24-96 h), high inoculum density (i.e. $10^{6}$ cells $/ \mathrm{ml}$ ), or high substrate concentration (i.e. $>10 \mathrm{mM}$ ). Assays may also be confounded by the indirect measurement of respiratory activity using redox sensitive dyes (e.g. tetrazolium dyes). This work describes a novel microtiterplate assay for physiological profiling based upon fluorometric detection of oxygen consumption due to microbial respiration. The BD Oxygen Biosensor System (BD Biosciences, Bedford, MA) utilizes a fluorophore whose fluorescence is quenched by the presence of oxygen. Maximum fluorescence was observed in as little as $5 \mathrm{~h}$ in substrate-adapted communities at substrate concentrations as low as $50 \mathrm{ppm}$. When coupled with methods for the molecular profiling of communities, this assay will provide a fundamental insight into the structural and functional dynamics of microbial communities.

\section{Molecular carbon in the Galaxy: new laboratory and observational studies \\ Richard Saykally • University of California, Berkeley}

The possible roles of two distinct forms of carbon in the ISM is being explored. Polycyclic Aromatic Hydrocarbon (PAH) molecules, proposed as UIR carriers are studied in the gas phase by IR emission spectroscopy, while pure carbon clusters are studied by a combination of IR cavity ringdown spectroscopy, FIR laser spectroscopy, and observational FIR astronomy.
We have developed an infrared photon counting method based on a blocked impurity band solid state photomultiplier (BIB-SSPM) for the purpose of measuring IR emission spectra of large PAH molecules under conditions approximating those in the interstellar medium. We have recently ${ }^{1}$ used this new technique (single-photon infrared emission spectroscopy, SPIRES) to measure gas phase IR emission from a number of UV laser-excited neutral PAHs over the entire spectral range of the UIRs. Apart from the $3.3 \mu \mathrm{m}$ feature, no acceptable matches with UIR features were found. Here, we describe the extension of SPIRES to PAH cations, generated and observed in a well-characterized ion beam.

We have reported ${ }^{2}$ the first observation of infrared emission from a gaseous ionic polycyclic aromatic hydrocarbon (PAH), the pyrene cation, over the range of wavelengths spanned by the UIRs. The complete set of pyrene cation IR emissions is observed, with relative intensities consistent with astrophysical observations, thus supporting the proposal that ionized PAHs are major contributors to the UIR bands. Additionally, unidentified features possibly arising from dehydrogenated PAH species are noted in the spectrum.

Neutral carbon clusters as large as C 13 have been studied in our laboratory by high-resolution IR diode laser spectroscopy. ${ }^{3}$ We have now developed a new IR cavity ringdown technique capable of higher sensitivity, much broader spectral coverage, and similar spectral resolution relative to a diode laser spectrometer, as demonstrated in a study of the $\mathrm{C} 9$ cluster. $^{4}$

Meanwhile, our initial detection of the $\mathrm{C} 3$ molecule in the source $\mathrm{Sg}$ B2 was confirmed by the ISO study of Charnicharo et al. demonstrating the potential for detecting such non-polar species via their low-frequency bending vibrations.

References

1 Wagner, D.R., Kim, H.-S. \& Saykally, R.J. (2000). Astrophys. J. 545, 854-860.

${ }^{2}$ Kim, H.-S., Wagner, D.R. \& Saykally, R.J. (2001). Phys. Rev. Lett. 86, 5691.

${ }^{3}$ Giesen, T.F., Berndt, U., Fuchs, G., Winnewisser, G., Provencal, R.A., Keutsch, F.N., Van Orden, A. \& Saykally, R.J. (2001). Proceedings of the Nobel Symposium 117 on The Physics and Chemistry of Clusters, eds Campbell, E.E.B. \& Larsson, M., pp. 309-310. Visby, Sweden.

${ }^{4}$ Giesen, T.F., Van Orden, A.O., Cruzan, J.D., Provencal, R.A., Gendriesch, R., Lewen, F., Winnewisser, G., Boreiko, R.T., Betz, A.L. \& Saykally, R.J. (2001). Astrophys. J. Lett. 551, L181-L184.

\section{ELISA rapid detection of life forms in Mars regolith simulant}

David Warmflash $\bullet$ NASA Johnson Space Center, George Fox $\bullet$ University of Houston, Carlton Allen $\bullet$ NASA Johnson Space Center, David McKay $\bullet$ NASA Johnson Space Center

We recently reported ${ }^{1}$ our application of enzyme linked immunosorbant assay (ELISA) to search for biomarkers in terrestrial soil samples, known to contain numerous life forms. Here we present our application of this technique to search for biological molecules in samples of Martian Regolith Simulant JSC Mars-1, volcanic ash from Hawaii ${ }^{2}$ that has been shown to have a biomass significantly lower than that of typical terrestrial soils. ${ }^{3}$ Assays utilizing each of four anti-nucleotide polyclonal antibodies all produced positive signals on $1: 10$ dilutions of JSC Mars- 1 extract as compared to controls. Signals produced when JSC Mars-1 samples were assayed were weaker than signals produced when soil was assayed under the same conditions. These results suggest that automated immunoassay devices have the potential of making significant contributions to the search for extraterrestrial life.

\section{References}

1 Warmflash, D., Larios-Sanz, M., Fox, G.E. \& McKay, D.S. (2002). 33rd Lunar and Planetary Science Conference, Houston, TX, March.

2 Allen, C.C., Morris, R.V., Jager, K.M., Golden, D.C., Lindstrom, D.J., Lindstrom, M.M. \& Lockwood, J.P. (1998). 29th Lunar and Planetary Science Conference, Houston, TX.

3 Allen, C.C., Griffin, C., Steele, A., Wainwright, N. \& Stansbery, E. (2000). 31st Lunar and Planetary Science Conference, Houston, TX, 2000. 


\section{Earth history}

\section{Rummaging through Earth's attic for remains of ancient life}

John Armstrong • University of Washington, Lloyd Wells $\bullet$ University of Washington, Guillermo Gonzalez • Iowa State University

We explore the likelihood that early remains of Earth, Mars, and Venus have been preserved on the Moon in high enough concentrations to motivate a searchmission. During the late heavy bombardment, the Earth must have experienced frequent large impacts. These types of impacts can eject material near the escape velocity that has the potential of landing and being preserved on the surface of the Moon. Retrieval and analysis of this ejecta would yield information on the geochemical and biological state of the early Earth. In addition to estimates for Martian and Venusian material, we calculate the amount of Terran material incident on the Moon over its history by considering the distribution of ejecta launched from the Earth by large impacts. We find, for a well mixed regolith, that the median surface abundance of Terran material roughly $10 \mathrm{ppm}$, corresponding to a mass of approximately $20000 \mathrm{~kg}$ of Terran material over a $10 \times 10 \mathrm{~km}^{2}$ area. We estimate the amount of material transferred from Venus and Mars to be 1 and $180 \mathrm{~kg}$, in the same search area. Given that the amount of Terran material is substantial, we estimate the fraction of this material surviving impact with intact geochemical and biological tracers, and suggest a search strategy for finding and retrieving it. Finally, we address the role of this material in re-seeding events of the early Earth.

\section{Nitrogen isotope models for Precambrian planktonic ecosystems}

Michael Arthur $\bullet$ Pennsylvania State University, Lee Kump $\bullet$

Pennsylvania State University

Nitrogen isotopes provide a potential method by which we may be able to document evolution of the planktonic ecosystem of Precambrian oceans. We have constructed a biogeochemical cycle model for oceanic nitrogen that is used to test assumptions regarding the effects of widespread deepwater and whole-ocean anoxia on nitrogen availability to plankton and nitrogen isotopic values for sedimented organic carbon. This model involves the interplay of denitrification, nitrification and nitrogen fixation, but does not explicitly consider the influence of tracemetal availability for requisite enzyme activity. Our simulations to date use a two-box ocean and predict whole-ocean averages for productivity, $\mathrm{N}$ and $\mathrm{C}$ burial and nitrogen isotopic compositions, but we are presently engaged in creating a multi-box ocean model.

One result of the modeling so far is that the nitrogen isotopic composition of organic matter is enriched in ${ }^{15} \mathrm{~N}\left(\Delta^{15} \mathrm{~N}>5 \%\right.$ o $)$ if oxygen is available initially in surface or deepwater masses, regardless of the amount of nitrogen fixation. This reflects the interplay of denitrification and nitrification that drive dissolved $\mathrm{N}$ towards enriched values even with high $\mathrm{N}$-fixation rates in surface waters. However, completely anoxic oceans produce organic $\mathrm{N}$ that is close to the nitrogen isotopic value assumed for atmospheric $\mathrm{N}_{2}$.

\section{Do paleosols constrain the $\mathrm{CO}_{2}$ level of the Archean atmosphere?}

Ekaterina Bazilevskaya $\bullet$ Pennsylvania State University, Hiroshi Ohmoto $\bullet$ Pennsylvania State University

Many recent investigators favor the $\mathrm{pCO}_{2}$ values constrained by Rye et al. (1995) for the Archean atmosphere. Their $\mathrm{pCO}_{2}$ values were estimated from the assumptions that greenalite, rather than siderite, was stable under the Archean atmosphere, and that the stability of these minerals depends primarily on $\mathrm{pCO}_{2}$. We have evaluated the stability of major Fe-bearing minerals as a function of $\mathrm{pCO}_{2}, \mathrm{pO}_{2}, \mathrm{pH}_{2} \mathrm{~S}, \mathrm{mFe}_{2}^{+}$, and $\mathrm{pH}$. Our study shows that the formation of greenalite, and any other ferrousrich minerals, requires extremely low $\mathrm{pO}_{2}\left(<10^{-50}\right)$ conditions. Since most recent investigators agree that $\mathrm{pO}_{2}>10^{-13}$ atm for the Archean atmospheric, ferrous-rich minerals could not have been stable under the Archean, as well as today's, atmosphere. The stable Fe-bearing mineral under the atmosphere has been ferric (hydr)oxide (goethite or hematite) throughout geologic time. At $\mathrm{pO}_{2}>10^{-50} \mathrm{~atm}$, siderite is unstable under any $\mathrm{pCO}_{2}$ condition; therefore, the absence of siderite in paleosols cannot be used to constrain the paleo atmospheric $\mathrm{pCO}_{2}$ level. Formation of siderite and other ferrous-rich minerals must have occurred in subsurface conditions where the $\mathrm{O}_{2}$ content of subsurface water decreased due to biological activity and/or reactions with ferrous-rich minerals.

\section{Sulfur isotope constraints on the rise of the atmospheric oxygen level in the Paleoproterozoic Andrey Bekker $\bullet$ Harvard University, H.D. Holland $\bullet$ Harvard University, D. Rumble $\bullet$ Geophysical Laboratory, Washington, DC, P.L. Wang • Geophysical Laboratory, Washington, DC, L.L. Coetzee •} Rand Afrikaans University

Mass independent fractionation (MIF) of the sulfur isotopes is extensive in sulfides and sulfates of sedimentary rocks $\geqslant 2.45 \mathrm{Ga}$ but not in rocks $\leqslant 2.0 \mathrm{Ga}$. Farquhar et al. (2000) argued that the loss of MIF between 2.45 and $2.0 \mathrm{Ga}$ is due to a dramatic increase in the oxidation state of the atmosphere. We have analyzed the isotopic composition of sulfur in early diagenetic pyrites from black shales in the upper Rooihoogte and lower Timeball Hill fms in South Africa. These units are older than the $2.22 \mathrm{Ga}$ Hekpoort basalt and significantly younger than 2.45 Gyr. The D33S values in the pyrites fall between -0.24 and $+0.28 \%(n=20)$. This range is much smaller than that observed in pre$2.45 \mathrm{Ga}$ sulfides and sulfates, suggesting that the oxygenation of the atmosphere had begun prior to the deposition of the Rooihoogte and Timeball Hill fms. This suggestion is supported by the presence of oölitic hematite deposits in the upper Timeball Hill fm. The $\delta 34 \mathrm{~S}$ values of pyrites which we have analyzed $(-33.2$ and $-25.6 \%$ ) are significantly more negative than those in older pyrites; the change is consistent with an increase in the sulfate content of seawater that probably accompanied the oxidation of the atmosphere. The Rooihoogte and Timeball Hill formations are sandwiched between two glacial diamictites, which may be the signature of glaciations that accompanied the oxidation of the atmosphere.

\section{Iron oxide may have filtered UV for early photosynthetic microbes \\ Janice Bishop • NASA Ames Research Center, Lynn Rothschild •}

NASA Ames Research Center

Solar radiation is a double-edged sword for photosynthetic organisms. While some wavelengths $(\sim 400-700 \mathrm{~nm})$ are critical for photosynthesis, ultraviolet (UV) radiation ( $<400 \mathrm{~nm}$ ) is usually harmful, even lethal. We have performed spectral analyses over the range $200-1000 \mathrm{~nm}$ of nanophase iron oxide suspensions in order to evaluate their spectral properties in solution in this region. Our initial results indicate that the iron-bearing minerals ferrihydrite and schwertmannite are able to filter the damaging UV rays, as hypothesized, while still allowing penetration of the necessary visible energy for photosynthesis. Preliminary growth experiments have shown that the photosynthetic protist Euglena can survive in environments containing the nanophase iron oxide minerals ferrihydrite and schwertmannite under solar UV radiation. For identical experiments without the iron oxide minerals, the Euglena did not survive. Spectral bands due to chlorophyll near $675 \mathrm{~nm}$ were found to be good indicators of healthy organisms in these experiments. The number and activity of the organisms was also determined using visual inspection with phase contrast microscopy at $400 \times$ magnification. If this technique proves to be successful for other organisms as well, then we may be able to explain links between early photosynthetic organisms and the mineralogy of their environment. 
Multiple carbon isotope excursions at the Permian-Triassic boundary separating two mass extinctions

Roger Buick $\bullet$ University of Washington, James A. Nicol $\bullet$ University of Sydney, Lori Watson $\bullet$ University of Sydney, David C. Catling • University of Washington

Rapidly deposited Permian-Triassic sediments from a non-marine foreland basin in eastern Australia show seven negative excursions in ${ }^{13} \mathrm{C}_{\text {org }}$ of up to $7 \%$. These fluctuations start in the late Late Permian at the extinction point of the Glossopteris flora and continue until the Permian-Triassic boundary as defined by palynology, which correlates with the peak of marine invertebrate mass extinction. As the isotopic excursions are not linked to changes in environmental setting, sedimentary regime, kerogen composition or plant community, they evidently resulted from major excursions in atmospheric ${ }^{13} \mathrm{C}$ and $/$ or $\mathrm{pCO}_{2}$. The pattern clearly does not reflect a single catastrophic event, such as an extraterrestrial impact, and carbon-cycle calculations indicate that neither massive volcanism nor methane-hydrate melting can adequately account for the size and frequency of isotopic perturbations. Instead, the data are more consistent with repeated overturning of a stratified ocean with anoxic deep-waters, expelling $\mathrm{CO}_{2}$ and $\mathrm{H}_{2} \mathrm{~S}$ that traumatized first terrestrial and then marine organisms, promoting separate mass extinctions in the two realms.

\section{Nitrogen fixation by coronae processes in the atmosphere over the Earth's geologic history} Nna Mvondo Delphine $\bullet$ Universidad Autonoma de Mexico, Ciudad Universitaria, Mexico, Navarro-Gonzalez Rafael $\bullet$ Universidad Autonoma de Mexico, Ciudad Universitaria, Mexico, Raulin Francois • Universites Paris, France, Coll Patrice • Universites Paris, France In the early Earth, abiotic nitrogen fixation was a fundamental prerequisite for the origin and sustainability of life until the advent of biological nitrogen fixation. While the relevance of lightning as a source of fixed nitrogen has been largely studied, the contribution of coronae discharges still remains unelucidated.

Here we present the first experimental simulation of atmospheric nitrogen fixation by point discharges in the ground over the whole geologic history. The energy yields of nitric oxide (NO), nitrous oxide $\left(\mathrm{N}_{2} \mathrm{O}\right)$ and nitrogen dioxide $\left(\mathrm{NO}_{2}\right)$ were estimated over a wide range of atmospheric compositions: $\mathrm{CO}_{2}-\mathrm{N}_{2}$ and $\mathrm{O}_{2}-\mathrm{N}_{2}$. In the absence of free oxygen, $\mathrm{NO}$ and $\mathrm{N}_{2} \mathrm{O}$ were the sole $\mathrm{N}$-products, whereas in the presence of oxygen $\mathrm{NO}_{2}$ was also formed. The maximum annual production rates of these three nitrogenated species were calculated to be lower than $10^{10} \mathrm{~g} \mathrm{~N} \mathrm{yr}^{-1}$ in both types of atmospheres, which indicates that corona discharges did not play a significant role in the overall pool of reactive nitrogen needed for the emergence and evolution of life.

\section{Critical reappraisal of the fossil evidence for life on} Earth prior to $3850 \mathrm{Ma}$, Akilia, SW Greenland

Christopher Fedo $\bullet$ George Washington University, Martin Whitehouse - Swedish Natural History Museum

Evidence for the oldest ( $>3850 \mathrm{Ma}$ ) life on Earth is claimed to be ${ }^{13} \mathrm{C}$ depleted graphite inclusions in apatite crystals hosted in banded quartzpyroxene rock from the island of Akilia, SW Greenland. This rock previously has been interpreted as banded iron formation (BIF), forming part of an inferred supracrustal succession with mafic volcanic rocks. Claims for a $>3850$ Ma age cannot be verified because no igneous contact with adjacent tonalites (now gneisses) can be shown. Field relationships on Akilia document multiple, intense deformation events that have resulted in parallel transposition of Early Archean rocks and significant boudinage, the tails of which form the banding in the quartzpyroxene rock. Geochemical data show distinct REE and other traceelement characteristics consistent with an ultramafic igneous, not BIF, protolith for this lithology and the adjacent amphibolites. Later metasomatic silica and iron introduction have merely resulted in a rock that superficially resembles BIF. An ultramafic igneous origin invalidates claims that the graphite inclusions represent evidence for life coeval with the late heavy asteroid bombardment, and cautions against the application of $\mathrm{C}$ isotopes as a unique biomarker for detecting past life.

\section{Stratigraphic and geochemical boundary conditions for Neoproterozoic glaciation in Northeastern Svalbard \\ Galen P. Halverson $\bullet$ Harvard University, Adam C. Maloof $\bullet$ Harvard}

University

We present stratigraphic, stable isotopic, and elemental data bracketing two established glacial units in the Polarisbreen Group (northeast Svalbard) and two older surfaces within the underlying Akademikerbreen Group that may represent cryptic glaciation. Insofar as the geochemical trends bracketing successive glaciations vary, these differences may prove useful in interbasinal correlations. Furthermore, in the absence of reliable syn-glacial proxies, the best means of constraining the geochemical evolution of the oceans during Neoproterozoic glaciations is to establish isotopic trends immediately prior to and following the ice age. Any model attempting to account for the cause, global extent, and duration of glaciation must be consistent with the boundary conditions imposed by these data. Post-glacial cap carbonates provide a robust geochemical record of the seawater upon deglaciation, though the potential influence of local hydrological and depositional variability must be considered. The record of the oceans leading up to glaciation is more difficult to construct due to common erosional truncation of sub-glacial strata. Establishing the stratigraphic context in which these records are preserved is crucial in assessing the limitations of the data while also possibly revealing the timing of the onset of continental glaciation relative to preglacial geochemical trends.

\section{Glimpse of a terrestrial snowball ocean: sea-floor dolomite-baritearagonite sequence in a Neoproterozoic cap carbonate, Northern Canadian Cordillera}

Paul F. Hoffman $\bullet$ Harvard University, Alexandra Van Dusen $\bullet$ Harvard University, Adam C. Maloof $\bullet$ Harvard University, C.A.

Ferguson • Arizona Geological Survey

The Ice Brook glaciation ( $c a$. $600 \mathrm{Ma}$ ) is the younger of two hypothesized snowball Earth events in NW Canada. During deglaciation, a transgressive cap carbonate (3-30 m thick) was deposited across a storm-dominated shelf. It begins with an extensive peloidal dolomicrite, followed at the shelf edge by limestone buildups composed primarily of fibrous cement, precipitated as aragonite on the sea floor. At the contact is a $6-\mathrm{cm}$ interval of macroscopic barite fans $\left(\mathrm{BaSO}_{4}\right)$, also clearly precipitated upon the sea floor. The micrite "rain" continued, undolomitized, after the barite layer. Because barite is extremely insoluble, the requisite Ba delivery depends on near-total reduction of seawater sulfate, a known inhibitor of sea-floor dolomitization. We infer that the dolomite-baritearagonite sequence reflects flooding of the shelf by anoxic, Ba-rich seawater evolved during the snowball event, and its interaction with oxic, meltwater-dominated, surface water. Oxidation of reduced sulfur caused barite precipitation and cessation of sea-floor dolomitization. The fibrous cement suggests that a nucleation inhibitor, possibly $\mathrm{Fe}(\mathrm{II})$, persisted in the bottom water but not at the surface where micrite was produced. Preliminary $\mathrm{C}$ and $\mathrm{O}$ isotope data are consistent with isotopic equilibrium between micritic dolomite and calcite, but disequilibrium between coexisting micrite and fibrous cement.

\section{Sulfur isotopes, snowball Earth events and the evolution of microbial sulfur species}

Matthew Hurtgen $\bullet$ Pennsylvania State University, Michael Arthur •

Pennsylvania State University, Lee Kump $\bullet$ Pennsylvania State

University, Anthony Prave $\bullet$ University of St. Andrews

Positive excursions in trace sulfate and pyrite associated with postglacial carbonates suggest that sulfur cycling in the late Neoproterozoic may have been strongly influenced by global glaciations. Additionally, an increase in the variability of the sulfur isotopic composition of pyrite 
in the Neoproterozoic has led some researchers to suggest that an increase in the oxygenation of the surface ocean facilitated the evolution of non-photosynthetic sulfide-oxidizing bacteria.

In this study, we have reconstructed the sulfur isotopic evolution of Neoproterozoic seawater sulfate by analyzing the isotopic composition of trace sulfate extracted from carbonates collected in Death Valley, CA, and Namibia. We present a record of carbonate-associated trace sulfate concentrations over the same time period. Our results indicate that the sulfur cycle behaved very differently in the early versus the late Neoproterozoic. More specifically, snowball Earth events forced highamplitude sulfur isotopic variations by decreasing oceanic sulfate concentrations and encouraging periods of intense weathering/oxidation. Furthermore, it is likely that 34 Spyrite-sulfate values were on average much smaller than $55 \%$, making it difficult to argue using sulfur isotopes that non-photosynthetic sulfide-oxidizing bacteria evolved in the Neoproterozoic.

\section{Laboratory growth of inorganic carbonate aggregates whose morphology is identical to the Apex chert nanofossils}

Stephen Hyde • Australian National University, Canberra, Nicholas Welham $\bullet$ Australian National University, Canberra, Andy Christy • Australian National University, Canberra, JuanManuel Garcia Ruiz • Granada University, Spain

The identification of the oldest putative nanofossils from the Apex chert deposit in Western Australia rests in part on their unusual morphology. Carbonate precipitates, grown in the presence of silica over hours, mimic those nanofossils in both size and form under certain conditions. The demonstrated possibility of exclusively inorganic "biomorphs" challenges the current identification of these structures as biological remnants.

\section{Predicting the methane concentration in the} Archean atmosphere based on ecological constraints Pushker Kharecha • Pennsylvania State University, James Kasting • Pennsylvania State University

Determining the $\mathrm{CH}_{4}$ concentration in the Archean atmosphere is important for understanding early Earth's climate and atmospheric evolution and could ultimately provide useful information for the search for Earth-like extrasolar planets. Previous studies have attempted to predict Archean $\mathrm{CH}_{4}$ concentrations based on thermodynamic considerations. Here, we take a kinetic approach instead. We will use a coupled ecological-photochemical model to estimate the amount of biogenic $\mathrm{CH}_{4}$ in the late Archean atmosphere $(2.5-2.8 \mathrm{Ga})$, based on environmental constraints such as nutrient availability. This will allow us to assess whether the biogenic $\mathrm{CH}_{4}$ concentration was limited more by thermodynamics or by ecological factors.

NASA's planned Terrestrial Planet Finder mission (TPF) will attempt to detect possible biogenic gases in the atmospheres of Earth-like extrasolar planets. Interpretation of the results from that ambitious endeavor will ultimately be based on comparison with known (and hypothetical) absorption spectra of biogenic gases in Earth's modern and primitive atmosphere. Our results can be used to generate hypothetical absorption spectra for an anoxic Archean atmosphere and thus could help determine whether early Earth-like planets could indeed be detected by TPF.

\section{The Late Ordovician mass extinction and Early Silurian recovery: comparison between Laurentian and global diversity}

Andrew Krug • Pennsylvania State University, Mark Patzkowsky • Pennsylvania State University

The evolution of complex life on Earth or any habitable planet may be closely tied to the number and severity of mass extinction events. In order to understand better the relationship between mass extinction and evolution, we began an analysis of the Late Ordovician mass extinction. We examined geographic variability in extinction and recovery patterns by comparing Late Ordovician and Early Silurian diversity in Laurentia to global diversity based on Sepkoski's unpublished compendium. Our Laurentian data consist of macrofaunal community lists. We limited our analysis to articulate and inarticulate brachiopods, trilobites, anthozoans, and bivalves.

Our preliminary results indicate that despite similar levels of extinction, diversity recovered more rapidly in Laurentia than globally. We also standardized our Laurentian data for sampling intensity by performing a rarefaction of genus occurrences per time interval. Results of this analysis suggest that diversity in Laurentia may have reached pre-extinction levels within 5 million years compared to 15 million years for global diversity. These results imply that with respect to diversity, the regional ecosystem recovered much more rapidly than the global ecosystem. However, the nature of this discrepancy may change once sample standardization of diversity has been performed on a global dataset.

\section{Ocean circulation during snowball Earth}

Lee Kump • Pennsylvania State University, Michael Arthur • Pennsylvania State University, Center Roberta Hotinski • Princeton/GFDL, David Pollard $\bullet$ Pennsylvania State Environment Institute

Previous workers have estimated the mean thickness of sea ice cover for the Neoproterozoic snowball Earth to be of the order of $1000 \mathrm{~m}$, based on assumed geothermal heat flow and climatological surface temperatures, although penetrating solar radiation may have caused much thinner tropical sea ice. We are refining these estimates in a $100 \%$ snowball Earth simulation, using the NOAA GFDL Modular Ocean Model coupled to an interactive sea-ice model. A continental reconstruction for $750 \mathrm{Ma}$ is used, and boundary conditions include surface temperature, insolation, and the net balance of precipitation and evaporation on the ice surface, all prescribed from a previous $750 \mathrm{Ma}$ experiment with an atmospheric GCM (GENESIS). Salt exclusion or dilution due to freezing/melting at the base of the sea ice provides an additional driving force for ocean circulation in addition to the buoyancy generated by the seafloor heatflow.

We are performing a number of sensitivity analyses of the model, including a test of the importance of thermal vs. haline forcing of circulation, the effect of ocean circulation on the thickness of sea ice cover, and the response to a non-uniform distribution of seafloor heatflow related to the position of mid-ocean ridges. Additionally, the model calculates primary productivity and dissolved oxygen distributions and evolution for scenarios with sufficiently thin sea-ice cover.

\section{Chemistry of the Mesoproterozoic ocean and links to biospheric oxygen levels}

Timothy W. Lyons $\bullet$ University of Missouri, Columbia, Anne M. Gellatly $\bullet$ University of Missouri, Columbia, Tracy D. Frank $\bullet$

University of Queensland, Brisbane, Australia, Linda C. Kah •

University of Tennessee, Knoxville

Recent geochemical models for the Proterozoic ocean-atmosphere system argue for levels of dissolved oxygen and sulfate that were intermediate with respect the oxygen-deficient conditions of the Archean and the oxygen-rich Phanerozoic. This progression is expressed in our detailed chemostratigraphic records from the Mesoproterozoic, wherein organic carbon sequestration and corresponding oxygen buildup manifest in long-term, carbonate $\mathrm{C}$ isotope trends. More specifically, a midMesoproterozoic transition to higher $\mathrm{C}$ isotope values occurred over an extended time interval in conjunction with an increase in total $\mathrm{C}$ isotope variability. We interpret these relationships to record a relative increase in the crustal inventory of organic $\mathrm{C}$, reflecting in part a locus of organic $\mathrm{C}$ burial that expanded to include anoxic deep marine sites where the preservation potential was high. The corresponding accumulation of oxygen in Earth surface environments associated with the increased organic C storage may have induced irreversible changes in the Mesoproterozoic biosphere.

Sulfur isotope records for the Proterozoic track the advent of quantitatively significant bacterial sulfate reduction within the marine realm. 
The availability of sulfate is linked directly to oxidizing conditions in the atmosphere and surface ocean and may have given rise to sulfidic anaerobic waters in the still poorly ventilated deep ocean. While $S$ isotope data for Mesoproterozoic sulfides and sulfates show the clear signs of widespread bacterial sulfate reduction generally lacking in the Archean, the abundance of isotopically heavy iron sulfides during this time period suggest a limited seawater sulfate reservoir relative to the Phanerozoic ocean. These lower sulfate concentrations may also be expressed in our high-resolution sulfide (pyrite and pyrrhotite) and sulfate (gypsum and carbonate-associated sulfate) $\mathrm{S}$ isotope trends, which show systematic short-term (100s of meters), large-magnitude variations of up to and exceeding 20 per mil. If global in origin, this style of large, comparatively rapid $\mathrm{S}$ isotope variability would require a Mesoproterozoic oceanic sulfate reservoir many times smaller than that of the Phanerozoic. Overall, these refined constraints on the Proterozoic transition in oxygen and sulfate availability, as also recorded in temporal distributions of bedded gypsum, are consistent with recent models for a highly redox stratified and sulfide-containing global Mesoproterozoic ocean.

\section{On the origin of Neoproterozoic sand wedge polygons \\ Adam C. Maloof $\bullet$ Harvard University, James B. Kellogg $\bullet$ Harvard University}

Thermal contraction cracking of permafrost produced sand-wedge polygons at sea level on the equator during late Neoproterozoic glacial episodes. Quaternary sand/ice-wedge polygons seem to require prolonged cooling periods of the order of days to weeks so that cold temperatures may penetrate to depths consistent with the observed crack lengths (of the order of $1 \mathrm{~m}$ ). We investigate whether large transient stresses associated with the diurnal temperature cycle during a snowball Earth event could have been responsible for sand-wedge growth. We conclude that temperature variations on the order of tens of degrees over 12-24 hour periods yield cracks one-half to several meters deep, similar to the lengthscale of sand wedges seen in the geological record.

\section{Tracing the action of water with igneous rocks}

Michelle E. Minitti • Arizona State University

Insight into an important portion of the Martian water budget is available in the most abundant rock type on Mars: igneous rocks. Igneous rocks provide constraints on the magmatic water reservoir of Mars, which is tied to the atmospheric and hydrospheric Martian water reservoirs through degassing of the planet. Studying the magmatic water reservoir using igneous rocks can therefore advance understanding of the presence and role of water throughout Martian history.

The activity of magmatic water can be traced with igneous rocks because water has a known and distinct effect on the major element chemistry of crystallizing melts. As a melt crystallizes, water dissolved in the melt facilitates iron oxide crystallization but suppresses feldspar crystallization. The influence of water on oxide and feldspar crystallization uniquely affects the $\mathrm{SiO}_{2}, \mathrm{FeO}$ and $\mathrm{Al}_{2} \mathrm{O}_{3}$ contents of the melts from which these phases form. Comparison of $\mathrm{SiO}_{2}, \mathrm{FeO}$ and $\mathrm{Al}_{2} \mathrm{O}_{3}$ contents of a Martian rock formed from a water-bearing melt to compositions of rocks created with and without water in the laboratory can establish whether water affected the formation of the Martian rock. The ability to detect the action of water in the major element chemistry of igneous rocks provides another tool with which future Mars missions, such as the Mars Exploration Rovers, can "follow the water."

\section{Life on Earth prior to $3.8 \mathrm{Ga}$ ? Resolving the age of the oldest known sedimentary rock}

Stephen J. Mojzsis • University of California, Los Angeles, T. Mark Harrison • The Australian National University, Canberra, Australia Crucial to understanding the conditions under which life emerged on Earth is determining the timing of its appearance. Isotopically light carbon found in $>3.8 \mathrm{Ga}$ metasediments (originally cherts, pelites and banded iron-formations) from West Greenland is suggestive of bio- logical activity in a marine environment but implies that metabolically sophisticated life had arisen prior to the end of a period of intense meteoritic bombardment of the Earth and Moon. This inference has been challenged on the basis that the interpretation of zircon geochronology from a crosscutting granitoid sheet, which establishes the minimum age of a metasedimentary enclave on Akilia (island), southern West Greenland as $>3.8 \mathrm{Ga}$, is incorrect. Instead, it is argued that the protolith to the orthogneisses formed earlier and that all zircons $>3.65 \mathrm{Ga}$ are inherited. We discuss the geochemical compositions of the rocks in question from West Greenland and report a U-Pb age depth-profile in a zircon from the same rock that permits direct assessment of the inheritance hypothesis. Our results reveal evidence for three phases of concentric zircon growth at 3.83, 3.6 and 2.7-2.5 Ga. Zircon growth at both $\sim 3.6$ and $2.7-2.5 \mathrm{Ga}$ is consistent with precipitation from a metamorphic fluid. As only the zircon core could have crystallized from the host rock, we conclude that the magmatic age of this rock is $3.83 \pm 0.01 \mathrm{Ga}$, therefore establishing a minimum age of the surface hydrosphere and life by that time.

\section{A methane-rich Proterozoic atmosphere: possible link to the Neoproterozoic snowball Earth glaciations?}

Alexander Pavlov $\bullet$ University of Colorado, Boulder, James Kasting • Pennsylvania State University, Matt Hurtgen $\bullet$ Pennsylvania State University, Michael Arthur • The Pennsylvania State University An enhanced atmospheric greenhouse effect is required throughout the Archean and Proterozoic to offset reduced solar luminosity. After the major transition of the atmospheric oxidation state at 2.0-2.3 Gyr, the photochemical lifetimes of reduced atmospheric gases should have been short. Therefore, a common view of the Proterozoic climate suggests that $\mathrm{CO}_{2}$ was the major greenhouse gas and that atmospheric $\mathrm{CH}_{4}$ concentrations were low.

However, $\mathrm{CH}_{4}$ could have still been the major greenhouse gas even in the Proterozoic atmosphere if methane fluxes were somewhat higher than at present. Here we argue that those fluxes were indeed higher because atmospheric oxygen and oceanic sulfate levels were lower than present in the Proterozoic.

Our 1-D photochemical model shows that the atmospheric methane mixing ratio is a highly nonlinear function of the surface methane flux. In our model, a factor of 10 increase in the methane flux results in a 60fold increase of the surface methane concentration. 1-D climate calculations show that such a high methane abundance would keep the mean global surface temperature well above freezing (despite lower solar luminosity), even if $\mathrm{CO}_{2}$ was present only at today's level.

Towards the end of Proterozoic, oceanic sulfate and atmospheric oxygen began to increase, which would have caused a decrease in methane production and possibly triggered Neoproterozoic snowball glaciation.

\section{Isotopic and geologic evidence for the nature of sulfur cycling during the Archean}

Bruce Runnegar $\bullet$ University of California, Los Angeles, Christopher

D. Coath $\bullet$ University of Bristol, James R. Lyons $\bullet$ University of

California, Los Angeles, Kevin D. McKeegan $\bullet$ University of

California, Los Angeles

Measurements of ratios of the four stable isotopes of sulfur $\left({ }^{32} \mathrm{~S},{ }^{33} \mathrm{~S},{ }^{34} \mathrm{~S}\right.$,

$\left.{ }^{36} \mathrm{~S}\right)$ using a combination of multi-collector and mono-collector methods on a Cameca ims 1270 ion microprobe have confirmed Farquhar et al.'s (2000) discovery that both sulfides (pyrites) and sulfates (barites) display large mass-independent fractionations throughout the whole of the Archean (2.5-3.9 Ga). A survey of samples from significantly different Archean environments that include shallow and deep water, sulfur-rich, hydrothermal systems may be used to constrain models for sulfur cycling during the Archean. It seems that atmospheric photochemistry produced both elemental sulfur and sulfate and that the former was reduced to sulfide by processes that are not yet well understood. There is no unequivocal evidence for bacterial sulfate reduction during this period of time. 


\section{Facies dependence of S-isotopic composition in pyrites from the $\sim 1.5 \mathrm{Ga}$ Roper Group, McArthur Basin, Northern Australia}

Yanan Shen $\bullet$ Harvard University, Andrew Knoll $\bullet$ Harvard University As part of a biogeochemical investigation of carbonaceous shales from the early Mesoproterozoic Roper Group, McArthur Basin, we identified systematic variations in the sulfur isotopic composition of early diagenetic pyrites along a paleoenvironmental gradient from inner shelf to deep basins. This pattern is consistent with hypotheses calling for low oxygen and sulfate levels in mid-Proterozoic oceans. Euxinic deep waters in the Roper seaway provided favorable sites for microbial sulfate reduction, producing sulfides with $\mathrm{S}$-isotopic values between -20 to +10 per mil. In a low sulfate water body, the preferential burial of ${ }^{32} \mathrm{~S}$ in the basinal sediments would drive isotopic values of dissolved sulfate in shelf waters to more positive values. Combined with the diffusion of sulfate from oxic water columns into sediments where inner shelf pyrite formed, this would be expected to result in progressive ${ }^{34} \mathrm{~S}$ enrichment of pyrites formed closer to the ancient shoreline. S-isotopic values as high as +50 per mil for inner shelf pyrites conform to this scenario. The implied long-term persistence of oceans with moderately oxic surface waters, low to moderate sulfate concentrations, and sulfidic deep waters has important implications for our understanding of Proterozoic biology.

\section{Development of the Proterozoic-Archean Hokkalampi weathering profile, Eastern Finland Sherry Stafford $\bullet$ University of Pittsburgh, Rosemary Capo • University of Pittsburgh, Brian Stewart $\bullet$ University of Pitsburgh, Jukka Marmo • Geological Survey of Finland, Hiroshi Ohmoto • Pennsylvania State University}

The Hokkalampi area in north Karelia, eastern Finland preserves a laterally extensive Proterozoic-Archean weathering profile ranging in thickness from 15 to $80 \mathrm{~m}$. The paleosol developed on $3.1-2.5 \mathrm{Ga}$ granitic basement rocks, and was buried by $>2.1 \mathrm{Ga}$ glaciogenic sediments; thus the soil-forming processes were operating during the proposed rise of atmospheric oxygen. Two locations that were cored and studied in detail exhibit depletion of $\mathrm{Ca}, \mathrm{Mg}, \mathrm{Al}$, and total $\mathrm{Fe}$ in the upper zone and enrichment of these metals in the lower zone. The chemical index of alteration also increases up profile. This could indicate leaching and mobility of cations, including $\mathrm{Fe}$, in the upper part of the soil and deposition with clay minerals at or near the paleowatertable. The Nuutilanvaara section is characterized by more oxidized, ironenriched material than the thicker Puaukkajanvaara section. Ferric/ ferrous ratios and $\mathrm{K}$-enrichment suggest that hydrothermal processes associated with greenschist metamorphism at 2.1 Ga could have affected the profile. Whole rock $\mathrm{Nd}$ and $\mathrm{Sm}$ isotope data from both localities generate an apparent isochron age of $2.34 \pm 0.23 \mathrm{Ga}$. This could represent reworked, fractionated basement material; however, micromorphologic analysis and field relationships suggest that pedogenic processes fractionated $\mathrm{Sm} / \mathrm{Nd}$, and the age represents the time of soil formation.

\footnotetext{
Absence of sterol biosynthesis in cyanobacteria Roger Summons • Massachusetts Institute of Technology, Linda Jahnke $\bullet$ NASA Ames Research Center, Kenneth Cullings $\bullet$ NASA Ames Research Center, Graham Logan • Geoscience Australia Reconstructing the hydrocarbon biomarker record of the early Earth requires us to make assumptions about the constancy and specificity of lipid biosynthetic pathways through geological time and rests upon our knowledge of the lipid composition of extant organisms. However, the validity of the steroid signature as a biomarker for Eukarya has been questioned because of some reports of "phytosterol" biosynthesis in cyanobacteria. Accordingly, we examined this issue in some new experiments with extant cyanobacteria grown in culture. After initial cultures of Phormidium and Chlorogloeopsis proved to contain sterols, in addition to the expected complement of hopanoid and 2-methylhopanoids, we hypothesized that they might be the result of contamination.
}

Subsequent cultures grown after repeated sub-culturing in the presence of cycloheximide proved to be devoid of sterol. Small subunit RNA sequence analyses indicated that many of our cultures were, in fact, contaminated with rust-like fungi and the sterol patterns were entirely consistent with the known sterol contents of these organisms. Our results are therefore in accord with the notion that sterol biosynthesis is, with very few exceptions, a primary characteristic of Eukarya. Consequently, the steroid hydrocarbons that are found in ancient sediments are most likely the remains of algae and other eukaryotes.

\section{Bacterial silicification and its relevance in astrobiological research}

Jan Toporski $\bullet$ Carnegie Institution of Washington, Washington DC, Andrew Steele $\bullet$ Carnegie Institution of Washington, Washington DC, Frances Westall $\bullet$ Centre de Biophysique Moléculaire, Orleans, France, Kathie Thomas-Keprta $\bullet$ NASA Johnson Space Center, David McKay - NASA Johnson Space Center

Evidence of life on Earth in the form of silicified microorganisms is reported from throughout the geological record as early as $3.5 \mathrm{Ga}$ ago to recent hot spring environments. Silicified microfossils are resistant to weathering which renders them readily preservable over long time spans. This is of particular interest in astropalaeontological research as if microbial life once was present outside Earth its traces may have become silicified. To better understand the mechanisms involved in silicification, laboratory experiments were designed to simulate silicification of bacterial biofilms. These were exposed to Si solution to test the influence of exposure time and $\mathrm{Si}$ ion concentration on silicification. The bacterial biofilms were subsequently analysed using transmission electron microscopy (TEM) in combination with energy dispersive Xray analysis. It was found that silicification commences after $24 \mathrm{~h}$ in $\mathrm{Si}$ solution and high ion availability results in better preservation of cellular detail; the concentration of Si thus is more important than the exposure time. Cells became permineralized and no amorphous silica precipitation was observed. High-resolution TEM studies revealed the presence of nanometer-sized crystallites in highly silicified cell walls. The design of this study will be used to monitor molecular alteration due to silicification to better understand biomarker formation.

\section{Mass spectrometric laboratory studies of tholin particles: application to early Earth and Titan} Melissa Trainer $\bullet$ University of Colorado, Boulder, Daniel Curtis $\bullet$ University of Colorado, Boulder, O. Brian Toon $\bullet$ University of Colorado, Boulder, Margaret Tolbert $\bullet$ University of Colorado, Boulder, Douglas Worsnop $\bullet$ Aerodyne Research, Inc., Christopher McKay $\bullet$ NASA Ames Research Center

Tholins are organic aerosols that are an important part of Titan's atmosphere, and it has been suggested that they may have been present on the early Earth. Reduced species in the ancient atmosphere, such as $\mathrm{CH}_{4}$, may have photolyzed to produce tholins, creating a ultravioletshield for the photolabile gases below and allowing these greenhouse gases to build up and warm the planet above freezing. Models that simulate the atmosphere of early Earth need to incorporate characteristics for such particles, such as optical constants, in order to correctly assess the shielding capabilities and antigreenhouse effect associated with a haze layer.

We have designed a flow system for producing tholin particles using various mixtures of gases that may have been present in the early Earth's atmosphere (namely $\mathrm{N}_{2}$ and $\mathrm{CH}_{4}$ ) and studying the properties of the synthesized particles. The study incorporates an aerosol mass spectrometer (AMS), which provides a novel analysis technique for the rapid characterization of these particles. The AMS provides instantaneous composition and size information, and our preliminary results show the compositional and size changes associated with various initial trace gas concentrations. The in situ size information for both Titan and early Earth tholins provides a new insight into the formation of these organic aerosols and their dependence on initial conditions. 
Investigations of the Mississippian-Pennsylvanian boundary in Central Texas

Melanie Urdiales $\bullet$ University of Houston-Downtown, James Coffield $\bullet$ Wheeling Jesuit University, Glen Merrill • University of HoustonDowntown, Marilyn Lindstrom $\bullet$ NASA Johnson Space Center

Reports of high levels of iridium at the Cretaceous-Tertiary boundary and the hypothesis that the anomalies could have been produced from the impact of a large meteorite has led us to investigate the recent discovery of several iridium and rare earth anomalies at the Mississippian-Pennsylvanian boundary in Central Texas.

Thirty-one samples were collected from J.R. Walker Ranch approximately $7 \mathrm{~km}$ southwest of Lampasas, TX. The samples were irradiated for 12 hours at the University of Missouri Research Reactor. Gammaray counting and mineral studies were performed at the Johnson Space Center's Instrumental Neutron Activation Analysis Laboratory and Xray Diffraction Laboratory.

Interference from other elements prohibited us from obtaining anything except upper limit values for Ir. However, several rare earth elements and noble metals were precisely determined using INAA. We identified an increase in rare earth and noble metal concentration coinciding with a change in mineralogy near the geological boundary. The older samples have less apatite and lower concentrations of rare earths and noble metals than the younger samples. It appears that the process responsible for concentrating the noble metals and rare earths is associated with the presence of phosphate-bearing minerals.

\section{Paleobiology of a 1640 Ma hydrothermal system: the McArthur River $\mathrm{Pb} / \mathrm{Zn}$ deposit}

Malcolm Walter $\bullet$ Australian Centre for Astrobiology; GEMOC Key Centre, Macquarie University, Junhong Chen $\bullet$ Australian Centre for Astrobiology; GEMOC Key Centre, Macquarie University, Stuart Graham • Australian Centre for Astrobiology; GEMOC Key Centre, Macquarie University, William Griffin $\bullet$ Australian Centre for Astrobiology; GEMOC Key Centre, Macquarie University, Graham Logan • Geoscience Australia, Roger E. Summons $\bullet$ Massachusetts Institute of Technology

The 1640 Ma McArthur River lead-zinc-silver deposit of northern Australia formed in a hydrothermal system. It has been intersected by more than 100 drill holes, and is exposed in an underground mine. It represents a superb opportunity to examine this class of ore deposit, and has been intensively studied.

Our work and most previous studies support the interpretation that the ore formed 10-20 m below the sediment-water interface in a marine environment below wave-base. It is possible to reconstruct the original fluid flow patterns and to determine thermal gradients, using relative abundances of $\mathrm{Cu}, \mathrm{Pb}$ and $\mathrm{Zn}$. Absolute temperatures are likely to range from ambient seafloor temperatures up to $250{ }^{\circ} \mathrm{C}$ or more.

Polycyclic aromatic hydrocarbons in ore and mudstone within the ore deposit show compound distribution patterns similar to those of hydrothermally-generated petroleum in the Guaymas Basin, Gulf of California, and different from those found in conventional oil. PAH abundances and isomer distributions confirm the interpreted temperature gradient between the source of mineralizing fluids and the sediments fringing the ore system. In situ LAM-ICPMS analyses of $\mathrm{Cu}$ and Fe isotopes in chalcopyrite and pyrite also show systematic variations along the flow path of the mineralizing fluid, becoming isotopically heavier with distance and declining $T$. This metal isotope pattern is likely to record both biotic and chemical effects but more work is required before these results can be fully interpreted.

Microfossils occur in chert that formed at the sediment-water interface, and in the ore and interbedded sediments. Previous studies have proposed that there are two populations in the chert: allochthonous cyanobacteria and possible microalgae, and benthic non-photosynthetic bacteria. The ore and interbedded sediments contain filamentous microfossils up to $60 \mathrm{~mm}$ wide, and spheroidal microfossils $7-14 \mathrm{~mm}$ wide with a single aperture.

We have analysed hydrocarbon biomarkers from the ore and associated sediments sampled on a centimetre to millimetre scale, and have also analysed the $\delta^{13} \mathrm{C}$ composition of selected hydrocarbons. The interpretation of some of the microfossils as cyanobacteria is supported by the presence of characteristic 2 methyl-hopanes. $C_{30}$ 24-n-propyl steranes occur in all samples, and their parent sterols are known only from marine Chrysophyte algae. All samples contain a $\mathrm{C}_{25}$ isoprenoid derived from Archaea. An unusual biomarker distribution found in some samples is similar to that of bitumen described from Neoproterozoic sediments in a previous study that combined sedimentological, micropaleontological, biomarker, and $\delta^{13} \mathrm{C}$ and $\delta^{34} \mathrm{~S}$ isotopic analyses to suggest that the hydrocarbon signal was derived from mats that contained sulfide-oxidising bacteria. $\delta^{34} \mathrm{~S}$ analyses indicate bacterial sulfate reduction.

This deposit is a rich source of paleobiological information, which in turn constrains the interpretations of ore genesis.

\section{Thick ice on the tropical oceans of snowball Earth: restriction of photosynthetic eukaryotes to isolated hotspots}

Stephen Warren $\bullet$ University of Washington, David Pollard $\bullet$ Pennsylvania State University, Richard Brandt $\bullet$ University of Washington

On the tropical oceans of the Neoproterozoic snowball Earth, snowfree sea ice would have existed in regions of net sublimation. Photosynthesis could have continued beneath this bare ice if it was thin and clear. By estimating the fluxes of latent heat and solar energy to be conducted upward through the ice, McKay (2000) concluded that the ice would have been only a few meters thick. That model assumed an excessive freezing rate at the base, and used a broadband radiation model which exaggerated the absorption depth of sunlight. When these aspects of the model are improved, we find that for a surface temperature of $-30{ }^{\circ} \mathrm{C}$, clean ice with albedo of 0.4 or greater would be at least $300 \mathrm{~m}$ thick.

Over the course of a snowball event, the low-latitude ocean surfaces would consist of: (1) bare sea ice with salt inclusions, (2) an evaporite deposit of hydrohalite, and (3) bubbly glacier ice emerging from equatorward ice-shelf flow. Their albedos would be $0.6-0.75$ (lower in dust-covered areas). Throughout most of the snowball event's duration, opaque surfaces and kilometer-thick ice would prevent sub-ice photosynthesis almost everywhere. Photosynthetic eukaryotes were therefore probably confined to isolated geothermal refugia where small pools of liquid water could exist locally at the surface. This may be one way in which the snowball episodes fostered evolution of diversity.

\section{Episodic developments of anoxic basins in the Late Archean, Early Proterozoic Oceans: evidence from $\mathrm{C} / \mathrm{P}$ ratios of shales}

Yumiko Watanabe $\bullet$ Pennsylvania State Astrobiology Research Center, Kosei Yamaguchi $\bullet$ Pennsylvania State Astrobiology Research Center, Hiroshi Naraoka $\bullet$ Tokyo Metropolitan University, Hiroshi Ohmoto $\bullet$ Pennsylvania State Astrobiology Research Center

It is well known that the $\mathrm{C} / \mathrm{P}$ ratios of organic matter and of bulk rocks in modern marine sediments greatly vary depending on the redox states of the overlying seawater: bulk-rock $\mathrm{C}_{\text {org }} / \mathrm{P}$ ratio of $\sim 70$ (mole ratio) in oxygenated environments and $\sim 700$ in anoxic environments. However, little has been known about the geochemical cycle of $P$ in the Precambrian. We have determined the major and trace element contents and isotope ratios in more than 300 shale samples, 3.3-2.1 Ga in age, from Australia, South Africa, and Canada. The $\mathrm{P}_{2} \mathrm{O}_{5}$ contents of these samples lie within a narrow range of $0.12 \pm 0.12$ (1 sigma), indicating the input and output fluxes of $\mathrm{P}$ in the Precambrian oceans were essentially the same as those in the Phanerozoic oceans. The bulk-rock $\mathrm{C}_{\text {org }} / \mathrm{P}$ mole ratio, however, exhibit a much larger variation, from $<10$ to $\sim 1000$. The variations in the $\mathrm{C}_{\text {org }} / \mathrm{P}$ ratio and other redox indicators (e.g. $\mathrm{S}$, $\mathrm{Mo}, \mathrm{U}, \mathrm{C}$ and $\mathrm{S}$ isotope ratios) suggest that the global oceans were basically oxygenated and local anoxic basins periodically developed during the Archean-Proterozoic time. The redox structures of the oceans and atmosphere, and the geochemical cycle of $\mathrm{P}$, appear to have been the same since at least $\sim 3.3 \mathrm{Ga}$. 


\section{Environment and evolution}

\section{Photochemical formation rates and optical properties of hydrocarbon aerosol haze through time-resolved, in situ laboratory measurements}

Mate Adamkovics $\bullet$ University of California, Berkeley, Kristie A. Boering • University of California, Berkeley

Photochemically generated hazes significantly alter radiative transfer in planetary atmospheres and can therefore critically affect both climate and habitability. On Titan and the giant planets, these hazes are known to be composed of hydrocarbon particulates that are photochemically formed from a mixture of gaseous precursors, yet the rates at which these formation processes occur for even the simplest systems has not been determined experimentally. Models of these atmospheres and of potential hazes in Earth's early atmosphere that may have served as a ultraviolet shield (thus protecting photo-labile greenhouse gases and organic compounds) include a number of approximations, some of them quite arbitrary, for the overall rate of photochemically driven particle formation. We have developed instrumentation for measuring the formation rates and optical properties of organic aerosols formed from irradiating mixtures of precursor gases via in situ optical $(633 \mathrm{~nm})$ scattering and online quadrupole mass spectrometry (1-200 amu). Results for $\mathrm{CH}_{4}$ and $\mathrm{CO}_{2} / \mathrm{CH}_{4}$ atmospheres will be presented.

\section{Provocative Mössbauer spectroscopy at the Permian-Triassic boundary: indication of an impact? David G. Agresti $\bullet$ University of Alabama at Birmingham, Thomas $J$. Wdowiak $\bullet$ University of Alabama at Birmingham \\ Mössbauer spectra (left) of a sample from the Meishan, China, Permian-Triassic (PT) boundary contact layer exhibits a temperature- dependent character like that observed for the Cretaceous-Tertiary (KT) boundary, ${ }^{1}$ thus suggesting the presence of a nanophase iron-rich layer as found at the KT boundary (see Fig. 1). This is not evident in the spectra (right) of the ash layer immediately above the PT contact. If the nanophase phenomenon at the PT contact is shown to be global, it would be consistent with an impact event. \\ References \\ ${ }^{1}$ Wdowiak et al. (2001). MAPS 36, 123-133.}

\section{Decoupling of coupled element cycles in microbial mats: origin of biogenic signatures in lithosphere and atmosphere \\ Laura Baumgartner $\bullet$ University of Connecticut, Daniel H. Buckley $\bullet$} University of Connecticut, Daniel R. Rogers $\bullet$ Woods Hole

Oceanographic Institute, Pieter T. Visscher $\bullet$ University of Connecticut Microbial mats are laminated sedimentary biofilms in which microorganisms live in close proximity. As a result, the cycling of major elements $(\mathrm{C}, \mathrm{N}, \mathrm{S}$, etc.) is very tight. The carbon cycle (from inorganic to organic carbon and back) needs additional redox species to function: $\mathrm{O}$ and $\mathrm{S}$ are the major ones in most microbial mats. For example, the electron donor for photosynthesis can be $\mathrm{O}$ (water) or $\mathrm{S}$ (reduced sulfur species) and respiration of organic $\mathrm{C}$ is facilitated through oxygen $(\mathrm{O})$ or sulfate or other partially oxidized sulfur species (S). If the conversion from inorganic to organic carbon is larger than the reverse, organic biomarkers in the lithosphere and reduced C-containing gases in the atmosphere would result. The opposite scenario, in which respiration prevails, could yield a variety of minerals including calcium carbonate as a permanent signature. We studied microbial mats in Baja California, Mexico and Highbone Cay, Bahamas, as part of the EMERG team to understand biogenic signatures. Dimethylsulfide (DMS) was studied as an atmospheric marker: DMS formation was a combined result of sulfate reduction (SR) and chemical reactions, whereas photochemical reactions accounted for most of the destruction of this gas. Production of calcium carbonate, a lithospheric marker, was mediated by SR, but different processes account for this process in Baja and Highborne.

\section{Iron isotope mass balance of the modern and ancient} Earth

Brian Beard $\bullet$ University of Wisconsin, Clark Johnson $\bullet$ University of Wisconsin

Iron isotope data obtained on several hundred natural samples to date suggest that markedly different $\mathrm{Fe}$ isotope fractionations will be produced in the modern, oxygenated Earth as compared to an oxygen-poor ancient Earth or perhaps Mars. In oxygen-poor environments segregation of $\mathrm{Fe}$ into an aqueous $\mathrm{Fe}(\mathrm{II})$ pool and a solid $\mathrm{Fe}(\mathrm{II}) / \mathrm{Fe}$ (III) pool can result in significant $\mathrm{Fe}$ isotope variations, as observed in chemically precipitated sediments such as banded iron formations. In the modern, oxygenated Earth, Fe cycling tends to be conservative and there is little isotopic discrimination. Clastic sediments including shale, turbidites, loess, suspended river load sediments and aerosols have a range of ${ }^{56} \mathrm{Fe} /{ }^{54} \mathrm{Fe}$ ratios similar to igneous rocks. Coupled with new high-precision analyses of high-temperature hydrothermal fluids from the Pacific and Atlantic midocean-ridge systems, which have low ${ }^{56} \mathrm{Fe} /{ }^{54} \mathrm{Fe}$ ratios, these results provide an estimate of the mass balance of $\mathrm{Fe}$ that is delivered to the modern oceans. Variations in the Fe isotope compositions of the oceans in the modern Earth appear to only be affected by the relative contributions of the hydrothermal and riverine/ aerosol components, or by cycling of the small percentage of $\mathrm{Fe}$ that is dissolved in seawater.

\section{Calibrating an animal molecular clock}

Jaime E. Blair $\bullet$ Pennsylvania State University, S. Blair Hedges $\bullet$ Pennsylvania State University

The diverse fields of geology, paleontology, and molecular biology intersect when establishing a timeline for the evolution of life on Earth. In this study we investigate the divergence times of three major animal groups (Vertebrata, Arthropoda, Nematoda) using the well-supported diapsidsynapsid (bird-mammal) split as a fossil calibration (310 Mya). Careful consideration is given to rate variation, sequence orthology, and other potential biases that may affect molecular clocks. The availability of vast molecular sequence data, including complete genomic sequences, allows this study to be more comprehensive than previous time estimates based on a limited number of genes. Initial results indicate that new divergence time estimates still greatly predate the Cambrian radiations observed in the fossil record, suggesting a long unrecorded history of animal evolution during the Neoproterozoic. Establishing divergence times within the animal kingdom will allow researchers to better understand the historical interactions between the evolution of life and major environmental events.

\section{Using the geologic and genomic molecular phylogenetic records to better understand the co- evolution of microbial metabolisms and the early Earth environment \\ Carrine Blank $\bullet$ University of California, Berkeley}

With the recent improvements in the resolution of the deep branching relationships in the tree of life using genomic sequences (Blank and Mishler, in preparation), a solid framework now exists upon which to test evolutionary hypotheses. One example is in the dating of the origin of oxygenic photosynthesis. The earliest recognizable cyanobacterial microfossils appear at $2.0 \mathrm{Ga}$, suggesting that the major bacterial radiation which gave rise to the proteobacteria, low $\mathrm{G}+\mathrm{C}$ gram positive bacteria, cyanobacteria, and green sulfur bacteria occurred just prior to this time.

This timing may be independently supported by the appearance of base-metal sulfide deposits at $1.8 \mathrm{Ga}$. It has been hypothesized that these deposits could not have been formed until the deep oceans became oxidized and sulfidic. Alternatively, it is possible that sulfidization was a result of the diversification of sulfate-reducing bacteria (SRBs). Indeed, most of the diversity of SRBs lies in the deepest divergences of this 

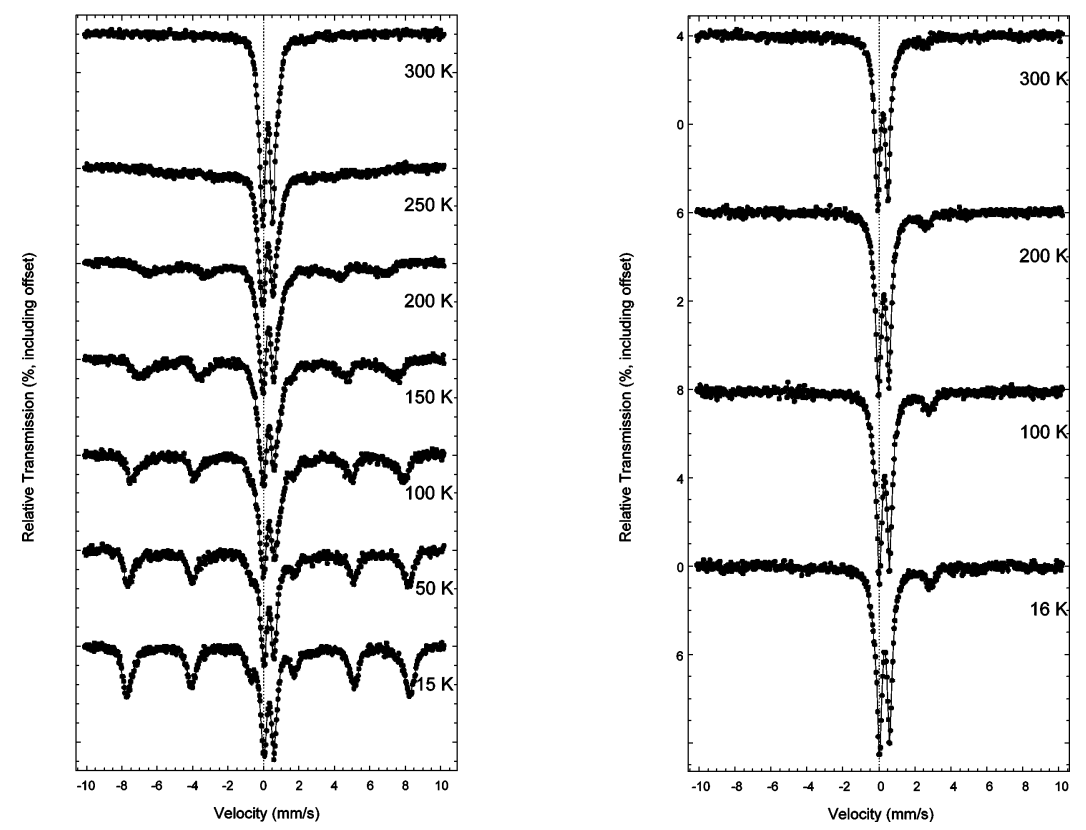

Fig. 1. Mossbauer spectroscopy at the Permian-Triassic boundary.

major bacterial radiation, so as oxidative photosynthesis arose, so also did the mesophilic SRBs.

Thus, the timing of the first appearances of base-metal sulfide deposits and recognizable cyanobacterial fossils, in conjunction with a wellresolved tree, suggests that oxygenic photosynthesis may have arisen much later in geological time than has been previously thought (perhaps around $2.2-2.0 \mathrm{Ga}$ ).

\section{Microanalysis of elemental, isotopic, and organic} chemistry of cellularly preserved fossils

Charles Boyce $\bullet$ Harvard University, George Cody $\bullet$ Carnegie

Geophysical Laboratory, Marilyn Fogel $\bullet$ Carnegie Geophysical Laboratory, Robert Hazen $\bullet$ Carnegie Geophysical Laboratory, Andrew Knoll $\bullet$ Harvard University

Permineralization can preserve fossils with cellular detail. The ability to analyze their preserved organic matter without the loss of spatial information would greatly increase the scope of paleobiological questions that can be investigated. A suite of microanalytical techniques has been adapted for the study of fossil organic matter: electron microprobe analysis providing micron-scale mapping of elemental abundances, continuous flow isotope ratio mass spectrometry linked to an elemental analyzer providing high resolution carbon isotopic abundances with millimeterscale spatial resolution, and X-ray spectromicroscopy performed on cellulose acetate peels of fossils providing micron-scale resolution of the distribution of organic functional groups. These microanalytical techniques have complementary susceptibilities to limitations such as the abundance of organic matter and its extent of degradation, the type of matrix mineral, and the relative destructiveness of the analysis. Initial research initiatives have focused on 300-400 million year old plant fossils, particularly the taphonomy of fossil organic matter and the evolution of vascular cell types in these earliest fossil land plants. With this system, investigation of important paleobiological questions can be balanced with control studies that make reference to the physiologies of related living organisms.

\section{Molecular fossils in Archean rocks: constraints on} the oxygenation of the upper water column Jochen J. Brocks $\bullet$ Harvard University, Roger E. Summons $\bullet$ Massachusetts Institute of Technology, Graham A. Logan $\bullet$ Geoscience Australia, Roger Buick $\bullet$ University of Washington

Several lines of evidence suggest that atmospheric oxygen levels in the Archean were much lower than at present, almost certainly $<1 \%$ PAL (present atmospheric level). However, molecular fossils extracted from
2.7-2.5 billion year old shales of the Fortescue and Hamersley Groups in the Pilbara Craton, Western Australia, indicate that the photic zone of the water column was at least weakly oxygenated. Firstly, the late Archean shales contain molecular fossils of bacteriohopanoids. Although hopanoid biosynthesis does not require oxygen, these lipids have never been isolated from strictly anaerobic bacteria. Secondly, the presence of cyanobacterial 2amethylhopanes and acyclic isoprenoids evidently derived from chlorophyll are convincing evidence for oxygen release within the photic zone. Thirdly, an unusual degradation pattern in the side chain of $3 b$-methyl-hopanes suggests that micro-aerophilic hetero-trophic bacteria, probably methano-trophs or methylo-trophs, inhabited a suboxic zone below a more oxygenated horizon. Finally, the presence of steranes in a wide range of structures with relative abundances like those from late Paleoproterozoic to Phanerozoic sediments is convincing evidence for the existence of eukaryotes in the late Archean. Sterol biosynthesis in extant eukaryotes requires dissolved molecular oxygen in concentrations equivalent to roughly $\sim 1 \%$ PAL. Thus, oxygen concentrations in Archean surface waters were probably high enough to support aerobic respiration.

\section{Investigation of local adaptation across an extreme environmental gradient: the relationship between genetic and environmental variation in a region of hybridization between two pupfish species endemic to Cuatro Cienegas, Mexico}

Evan W. Carson • Arizona State University, Thomas E. Dowling • Arizona State University

The Cuatro Cienegas basin, Mexico, contains a remarkable diversity of aquatic environments, including physicochemically constant thermal springs, environmentally severe terminal sinks (playa lakes and marshes), and connecting regions of intermediate environmental variability. Because two endemic pupfish species, Cyprinodon bifasciatus and C. atrorus, are adapted to the opposite environmental extremes, but hybridize extensively in environmentally intermediate mid-reaches, this system provides and excellent opportunity to investigate processes of local adaptation across an extreme environmental gradient. To address the relationship between genetic and environmental variation within a contemporary hybrid zone, we are conducting surveys across seasons at 14 sites spanning the width and length of the Rio Churince system. Comparison was made between spatial and seasonal variability in aquatic physicochemical characteristics ( $\mathrm{pH}$, salinity, specific conductivity, and temperature) and frequency of species-specific nuclear alleles 
(CK, RAG 1, and TPI-B). Preliminary evidence indicates spatial and seasonal association between $C$. atrorus alleles and fluctuating, extreme environments.

\section{Are nanobacteria alive?}

\section{Neva Ciftcioglu $\bullet$ NASA Johnson Space Center}

We discovered a novel proteinaceous nidus in kidney stones, obtained monoclonal antibodies detecting the target and demonstrated that these nidi have metabolic activity. The nidus was culturable in cell culture medium and appeared to contain lipopolysaccharide. At that point, the name "nanobacteria" (NB) was adopted though it is not yet clear whether nanobacteria is the right term for the finding. NB are so small that there is not enough space for a standard sized genome. However, the ultimate significance of "NB phenomena" may not dependent on whether it represents an independent, free-living organism. What might be behind all our observations: minerals can replicate crystals. They are often used as catalysts in nanotechnology, and may bind polymers like proteins, lipids, and nucleic acids creating assemblies reminiscent of life forms. It has been proposed that mineral structures and mineralmediated catalysis may have played a main role in starting the life on Earth. It thus appears that minerals may have both structural and functional capabilities to replicate. How far can that go? Can life be created from nonliving materials? What are the simplest forms of life? NB may represent such nonliving but replicating mineral formations capable of performing some life-like functions.

\section{Recovery in impact craters: an extreme case of primary succession}

C.S. Cockell $\bullet$ British Antarctic Survey, P. Lee $\bullet$ NASA Ames Research Center, G. Osinski $\bullet$ University of New Brunswick, Fredericton, Canada

Compared to other agents of localized ecological destruction, impact events are extreme, generating temperatures and pressures that effectively remove biogenic elements and compounds from the impact substrate. This will particularly be the case for melt rocks formed within a crater. The impact melt rocks of Haughton crater in the Canadian High Arctic are colonized with a vegetation cover $<2 \%$, about half that of the surrounding polar desert, but in specific locations the activity of burrowing lemmings facilitates the formation of productive mounds of vegetation - "micro-oases."1 Although there is no fresh crater on Earth with which to study immediate post-impact succession, the microoases represent one generalized mechanism for post-impact succession on impact melt rocks. Parallels are recognized with post-volcanic succession on tephra observed at Mt St Helens. In the case of large impacts $\left(\sim>4 \times 10^{22} \mathrm{~J}\right)$, succession within the crater will be influenced by succession occurring outside the crater because of the feedback of global environmental changes. In the case of impacts with an energetic yield $<5 \times 10^{16} \mathrm{~J}$, for which there is only an airburst, succession will be mainly limited to secondary succession (e.g. Tunguska). Within the context of scaling effects, the data from present-day craters can be used to develop a picture of the nature of post-impact local succession.

\section{References}

${ }^{1}$ Cockell, C.S., Lee, P., Schuerger, A.C., Hidalgo, L., Jones, J.A. \& Stokes, M.D. (2001). Microbiology and vegetation of micro-oases and polar desert, Haughton Impact Crater, Devon Island, Nunavut, Canada. Arctic, Antarctic and Alpine Research 33, 306-318.

\section{Laser Raman spectroscopic analysis of biochemical changes caused by fossilization}

Andrew D. Czaja $\bullet$ University of California, Los Angeles, J. William Schopf $\bullet$ University of California, Los Angeles, Michael C. StorrieLombardi $\bullet$ NASA Jet Propulsion Laboratory, Anatoliy B. Kudryavtsev - University of Alabama, Birmingham, Rohit Bhartia $\bullet$ NASA Jet Propulsion Laboratory

During diagenesis and fossilization, the organic constituents of living systems are degraded and geochemically altered. A thorough understanding of how such constituents change over time would allow us to extrapolate, from fossil evidence, aspects of the original biochemistry.

Toward this end, we have used both UV- and visible light-laser Raman spectroscopy (LRS), to analyze the organic chemical com- position of a modern fern species (Dennstaedtia cicutaria) and a well preserved fossil analogue (Dennstaedtiopsis aerenchymata) petrified in the Eocene Clarno Chert of Oregon. Raman spectra of these plant axes (rhizomes and rachises), whose visibly distinct tissues (xylem, phloem, cortex, epidermis, etc.) have differing original biochemistries, show evident differences among the various tissues of any given axis and also between the modern and fossil specimens - the former reflecting biochemical differences; the latter, changes caused by fossilization.

Studies are underway including heating experiments, using the modern ferns, to simulate the degradation caused by geological processes. Samples are to be heated under anoxic conditions to different stages of degradation, producing a gradient of artificial "fossils" from modern to the level of fossilization seen in the Eocene specimens, and analyzed with LRS to better understand the natural changes in biochemistry through time.

\section{Ribosome origins}

George E. Fox $\bullet$ University of Houston, James Hury $\bullet$ University of Houston, Uma Nagaswamy • University of Houston, Maia Larios-Sanz - University of Houston

Modern organisms utilize ribosomes to synthesize defined sequence peptides of a single chirality. Genomic comparisons have revealed that all three kingdoms of life share a similar machinery and structure/ function studies reveal that it to be an RNA machine. These findings suggest that an essentially modern ribosome was already present when the three kingdoms diverged and therefore probably had its origins in the RNA world. We are identifying features that provide insights into the relative time of origin of various ribosomal components. Such timing events are used to assemble cogent hypotheses about ribosome origins and thereby obtain insights into this very early phase in biological evolution. In the work described here, we consider the consequences of the hypothesis that the oldest regions of the structure will be most tightly integrated into it. We have examined all the RNA/RNA contacts between distant regions in the RNA. The results show that Domain 5 is the most strongly integrated region of the RNA and therefore is likely to be the oldest portion. This is followed by either Domain 2 or 4 in the 23S rRNA and then Domain 2 of the $16 \mathrm{~S}$ rRNA. The reasonableness of these results is independently confirmed by the fact that Domain 5 catalyzes the synthesis of the peptide bond, a capability which must have been present at the very beginning.

\section{High oxygen events in the history of the Earth's atmosphere, with special emphasis on the end- Cretaceous: effect on plants and animals \\ Joe Gale $\bullet$ Hebrew University of Jerusalem, Israel, Shimon Rachmilevitch $\bullet$ Hebrew University of Jerusalem, Israel, Joseph Reuveni $\bullet$ Hebrew University of Jerusalem, Israel}

The cyanobacteria first appeared around 3 ByBP. They and later plants changed the composition of the atmosphere, from traces of oxygen to today's $21 \mathrm{kPa}$ and from high $\mathrm{CO}_{2}$, to the pre-industrial $27 \mathrm{~Pa}$. The high $\mathrm{O}_{2}$ reduced plant photosynthesis, inhibited nitrogen fixation and enabled the evolution of herbivores and carnivores. However, these changes in the composition of the atmosphere were not uniform in time. In addition to sporadic reversals in the trend of $\mathrm{CO}_{2}$, there were short events (millions of years) in which oxygen rose to as high as $40 \mathrm{kPa}$.

In this study, plants were grown under the simulated high $\mathrm{O}_{2}$ of the end-Cretaceous (65 MyBP) atmosphere $\left(\mathrm{O}_{2}\right.$ of $28 \mathrm{kPa}$ and $\mathrm{CO}_{2}$ between 30 and $60 \mathrm{~Pa}$ ). Growth of $\mathrm{C}_{3}$ plants was significantly reduced compared with growth under today's atmosphere. By extrapolation, this reduction would have been even greater following the period preceding the endCretaceous, when $\mathrm{CO}_{2}$ was much higher than that of today. This would have negatively impacted dependent animal life. Such a deleterious ecological chain effect may have been a significant causal factor of the C-T boundary extinctions, in addition to the known effects of the extensive volcanism and bolide impact of that time.

Conversely, $\mathrm{C}_{4}$ plants were found here to be relatively insensitive to high $\mathrm{O}_{2}$, suggesting an additional environmental driver for their evolution. 
Detecting functional divergence among proteins using evolutionary and structural genomics analyses

Eric Gaucher • University of Florida, Scripps, Steven Benner • University of Florida, Scripps

Outside the field of molecular evolution, only simple evolutionary approaches (e.g. homology searches) are widely used today to infer "function." Only recently, however, have serious efforts been devoted to the development of mathematical treatments that maximize the interpretive value of genomic sequences. The complex interplay between sequence, folded structure, and function in a divergently evolving protein family is reflected in the rate at which individual sites suffer amino acid replacement. Thus, detecting shifts in the evolutionary rates of individual sites offers a way to identify potential instances of functional divergence among proteins. The covarion model was among the first to consider protein structure and function in this way. The ability to detect rate shifts at sites relies on molecular evolutionary approaches, whereas functional explanations for these changes can be derived from experiments in structural biology. Two years ago at AbSciCon, we demonstrated the potential utility of this type of analysis towards the generation of hypotheses of functional divergence at the sequence level. This year, we will show how recent experimental data support some of our hypotheses, while also permitting us to refine other hypotheses. This combination of experimental and computational biology defines a powerful approach to understanding the mechanisms of molecular evolution.

\section{Sulfur and carbon isotope fractionation during lithotrophic growth by the hyperthermophilic sulfate reducer Archaeoglobus sp.}

Kirsten Habicht • University of Southern Denmark, David Des Marais - NASA Ames Research Center, Linda Jahnke • NASA Ames Research Center

The early life on Earth may have evolved in hydrothermal environments. These areas often have a high efflux of $\mathrm{H}_{2}$ that can serve as substrate for sulfate-reducing organisms. The activity of sulfate-reducing organisms can be identified in sedimentary rocks through the stable sulfur isotopes of sedimentary sulfides and sulfate. Likewise the microbial fixation of carbon into organic matter can be identified through the stable carbon isotope of these compounds. The extent of sulfur and carbon isotope fractionation during lithotrophic sulfate reduction is not well known and we have therefore investigated Archaeoglobus grown in a chemostate at $80^{\circ} \mathrm{C}$ with $\mathrm{H}_{2}$ and $\mathrm{CO}_{2}$ as substrates. During both lithoheterotrophic sulfate reduction (with $\mathrm{H}_{2}, \mathrm{CO}_{2}$ and acetate) and lithoautotrophic sulfate reduction (with $\mathrm{H}_{2}$ and $\mathrm{CO}_{2}$ ) we measured a sulfur isotope fractionation of about 20 per mil. This is a similar $\mathrm{S}$ isotope signature to organotrophic communities. We measured no carbon isotope fractionation during lithoheterotrophic fixation of carbon whereas a fractionation of up to 14 per mil was measured during lithoautotrophic growth. Also the carbon isotope fractionation between the DIC and the two most common lipids of Archaeoglobus, archaeol and tetraethers, showed a large difference when Archaeoglobus was grown lithoheterotrophic and lithoautotrophic.

\section{A mission to really early Earth: determining when conditions suitable for life emerged on Earth} T. Mark Harrison • Australian National University; University of California, Los Angeles, Stephen J. Mojzsis $\bullet$ University of California, Los Angeles; University of Colorado, R.T. Pidgeon • Curtin University, T.R. Ireland • Australian National University, Vickie Bennett • Australian National University, M. Honda $\bullet$ Australian National University, Bernard P. Bourdon • Institut de Physique du Globe, J.L. Kirschvink $\bullet$ California Institute of Technology, F.J. Ryerson $\bullet$ Lawrence Livermore National Laboratory, A. Berry • Australian National University

When did conditions suitable for the habitation of Earth first appear? Since the necessary energy source and organic building blocks were available during the earliest moments of planetary evolution, this question reduces to: when did suitably quiescent conditions and liquid water first occur at or near the Earth's surface? The earliest direct evidence of a hydrosphere is in the form of $\geqslant 3.82 \mathrm{Ga}$ marine sediments from West Greenland. That these rocks contain carbon isotopic evidence of the existence of relatively sophisticated biological activity raises the possibility that life emerged during the Hadean era (4.5-4.0 Ga), a period for which there is no known rock record. This being the case, how can we determine whether life, or an environment conducive to life, was extant during this era? The record that is preserved is in the form of Hadean detritus deposited in younger sedimentary basins. The discovery of $\geqslant 4.3$ Ga detrital zircons from Jack Hills, West Australia, offers the prospect of gaining unprecedented insights into surface environmental conditions during the earliest phase of the Earth's evolution. For example, oxygen isotopes from these ancient zircons suggest the presence of a terrestrial hydrosphere and stable continents only 200 Myr after accretion - both conditions favorable for the emergence of life. These results challenge the traditional view that continental formation and the development of a hydrosphere were frustrated by meteorite bombardment and basaltic igneous activity until 3.9-4.0 Ga. In addition to assess the timing and mechanism of hydrosphere's origin, we have embarked upon age characterization of $>104$ Jack Hills zircons in order to gain an understanding of other key geophysical events in the earliest history of the planet.

\section{Using homolog groups to create whole-genomic phylogenetic trees \\ Christopher H. House $\bullet$ Pennsylvania State University, Bruce Runnegar} - University of California, Los Angeles, Sorel Fitz-Gibbon • University of California, Los Angeles

Evolutionary relationships can be studied using methods based on the presence and absence of genes in whole genome sequences. Several different methods have been proposed and can be divided into two broad categories: those based on the presence and absence of suspected ortholog pairs or the "ortholog method" and those based on the presence and absence of gene families or "the homolog method."

We will present our latest results in the development of the "homolog method", which include an analysis of the genomic relationships of 52 prokaryotic organisms. Our analyses continue to show that small or derived genomes (which are not typical of the ancestral state) can adversely affect the results. However, removal of such genomes from our analysis results in trees that are remarkably similar to trees obtained by analysis of rRNA, the "ortholog method," or the phylogenetic analysis of multi-gene datasets. The trees are robustly supported by bootstrap values, consistency indices, decay indices, and by consistency across differing $z$-score cut-offs defining a match between protein sequences.

We will also present results from our analysis of the genomic relationships between five eukaryotes, results which bear upon the highly controversial placement of the Nematoda phylum with respect to other animal phyla. Our homolog-based tree supports a Coelomata clade rather than an Ecdysozoa clade.

\section{The origins of metazoan complexity: is reproductive signaling antecedent to sensory \& neural organization? \\ David K. Jacobs $\bullet$ University of California, Los Angeles, Ruth D. Gates} - University of California, Los Angeles

We explored the POU/homeodomain containing developmental genes down the tree of life, including homologues of: vertebrate Brain3; the pituitary specific gene, Pit1; and a neural marker Brain1. The statocyst and eye of the jellyfish express Brain3, consistent with this genes function in bilaterian sense organ development. Even more surprising is our recovery of Pit1 from cnidarians, ctenophores, and sponges. This gene, absent from the nematode and fly, functions in the pituitary development of chordates. The pituitary evolved from an external organ that received water-borne reproductive signals. We find Pit1 expressed in sensory tracts leading from the oral opening to the gonads in jellyfish consistent with a pituitary antecedent. Gene-tree analysis supports a basal position for Pit1 among metazoan POU genes, followed by the divergence of 
Brain3 and Brain1. This suggests a scenario where: (1) a Pit1-like gene functioned in reproductive signaling prior to the divergence of all living Metazoa; (2) gene duplication produced Brain 3 associated with the evolution of sense organ precursors in the metazoan stem; (3) further gene duplications led to Brain 1 and other POU genes that were coopted for roles in development of an evolving nervous system. Thus, reproductive signaling may have been the precursor to the evolution of metazoan sensory and neural organization.

\section{Quantifying Fe isotope biosignatures}

Clark Johnson • U.W. Madison, Brian Beard • U.W. Madison, Sue Welch $\bullet$ U.W. Madison, Dianne Newman $\bullet$ California Institute of Technology, Laura Croal $\bullet$ California Institute of Technology, Ken Nealson • University of Southern California

Building upon our initial proposal that $\mathrm{Fe}$ isotopes may be a useful biosignature in the search for life on the early Earth or other planets, we have developed a sufficient database for experiments in the system $\mathrm{Fe}(\mathrm{II})-\mathrm{Fe}(\mathrm{III})$-ferric (oxy)hydroxide in both inorganic and biologic systems so that the isotopic effects of biological processing of Fe may be quantified for the first time. Determination of equilibrium (inorganic) $\mathrm{Fe}$ isotope fractionation factors at low temperatures indicates that the ${ }^{56} \mathrm{Fe} /{ }^{54} \mathrm{Fe}$ ratios are higher in $\mathrm{Fe}(\mathrm{III})$, relative to $\mathrm{Fe}(\mathrm{II})$, by 2.7 per mil, whereas the ${ }^{56} \mathrm{Fe} /{ }^{54} \mathrm{Fe}$ ratios of $\mathrm{Fe}(\mathrm{III})$ are 0.2 per mil lower than those of hematite. From these relations, we can calculate the $\mathrm{Fe}(\mathrm{II})$-hematite $\mathrm{Fe}$ isotope fractionation factor, which may be directly compared to new experiments involving Fe-reducing bacteria, as well as photosynthetic Fe-oxidizing bacteria. The striking results of these new biological experiments is that the ferric-ferrous $\mathrm{Fe}$ isotope fractionations lie consistently between +1.2 and +1.5 per mil, which is approximately half that of the abiologic systems; we interpret this contrast to be the $\mathrm{Fe}$ isotope "vital" effect. These results suggests that the isotopic fingerprint of, for example, photosynthetic Fe-oxidizing bacteria would be moderately high ${ }^{56} \mathrm{Fe} /{ }^{54} \mathrm{Fe}$ ratios for hematite, which are observed in Archean banded iron formations.

\section{Basic conditions for transition of biological into intelligent life \\ V.N. Kompanichenko $\bullet$ Institute for Complex Analysis, Russia} The main planetary and geological conditions, which provide successful advancement of life from the simplest to intelligent level, have been considered. Having appeared, living beings displayed an inevitable tendency to evolve. Under the following favorable conditions an intelligent civilization has a good chance of developing from primary forms of life on a planet: (1) a very long period of favorable temperature conditions, which are necessary for laying the foundations of life on a planet, depending mainly on planetary orbital characteristics; (2) a comparable ratio of ocean and land on a planet (optimal thickness of the hydrosphere) that makes possible initial evolution of life in the ocean and its further evolution on land; (3) sufficient starlight and optimal clouds (rain) that allow growth of plants, transforming light energy of the star into biochemical energy of the biosphere.

\section{The protective roles of the antioxidant enzymes superoxide dismutase and catalase in the green photosynthetic bacterium Chloroflexus aurantiacus Vanessa Lancaster • Arizona State University, Robert Blankenship • Arizona State University, Lynn Rothschild $\bullet$ NASA Ames Research Center}

Living organisms are constantly bombarded by cell-damaging reactive oxygen species (ROS). Sources of ROS include ionizing radiation, normal cell metabolism, and metal catalyzed oxidation systems. Modifications of proteins, DNA, and lipids caused by ROS are lethal and cells have had to evolve efficient defense mechanisms to survive.

The antioxidant enzymes superoxide dismutase and catalase in the photosynthetic green bacterium Chloroflexus aurantiacus are the focus of this study. Chloroflexus aurantiacus is an evolutionarily significant organism since it groups in the first branch of the eubacteria, which are capable of photosynthesis. This early organism is found growing in mats beneath cyanobacteria in the hot springs of Yellowstone National
Park. This environment is both hyperoxic and high in ultraviolet radiation, a potent source of ROS.

The superoxide dismutase and catalase genes from Chloroflexus aurantiacus are currently being cloned and the properties of the proteins are being characterized. The patterns of activity of these enzymes have recently been profiled in the alkaline mats of Yellowstone. These enzymes were shown to exhibit inhibited activity under conditions of oxidative stress. Enzymatic activity was also shown to recover and to be sustainable during the day when exposure to oxidants occurs early, suggesting possible gene inducibility.

\section{Resistance of virus to extinction upon bottleneck passages: study of a decaying and fluctuating pattern of fitness loss}

Ester Lázaro • Centro de Astrobiología (CSIC/INTA), Cristina Escarmís • Universidad Autónoma de Madrid, Spain., Juan PérezMercader • Centro de Astrobiología (CSIC/INTA), Susanna C.

Manrubia $\bullet$ Centro de Astrobiología (CSIC/INTA), Esteban Domingo - Centro de Astrobiología (CSIC/INTA); Universidad Autónoma de Madrid, Spain

RNA viruses display high mutation rates and their populations replicate as dynamic and complex mutant distributions termed viral quasispecies. Repeated genetic bottlenecks, which experimentally are carried out through serial plaque-to-plaque transfers of the virus, lead to a fitness decrease (a diminished capacity to produce infectious progeny). Here we report on an analysis of fitness evolution of several low fitness footand-mouth disease virus clones subjected to 50 plaque-to-plaque transfers. Unexpectedly, the fitness decrease, rather than being continuous and monotonic, displayed a fluctuating pattern that was influenced by both the virus and the state of the host cell. The amplitude of the fluctuations increased as the fitness decreased, resulting in a remarkable resistance of the virus to extinction. While the frequency distribution of fitness in control (independent) experiments follows a log-normal distribution, the probability of fitness values in the evolving populations fitted a Weibull distribution. We suggest that multiple functions of viral genomic RNA and its encoded proteins, subjected to high mutational pressure, interact with cellular components to produce this non-trivial, fluctuating pattern. Our results imply that the complexity of mutational events in a simple organisms like a virus results in considerable resistance to extinction.

\section{Impact-induced lithic microbial habitats at Meteor Crater, Arizona \\ Pascal Lee $\bullet$ SETI Institute; NASA Ames Research Center, Ted E.} Bunch $\bullet$ Northern Arizona University; US Geological Survey, Charles S. Cockell • British Antarctic Survey, Chris P. McKay $\bullet$ NASA Ames Research Center

We report here on finding evidence for lithic microbial colonization in shocked rocks at Meteor Crater, AZ, and for variations in the degree of colonization as a function of shock level. The patterns of colonization at Meteor Crater are different from those observed previously at Haughton Crater, Devon Island, Arctic Canada (Cockell \& Lee 1999, 1st. Astrob. Sci. Conf.). In the gneisses at Haughton, we had observed the following simple trend: the higher the shock level, the greater the fracturing and vesiculation, and the more enhanced the lithic microbial colonization.

The Moenkopi sandstone and Kaibab limestone and dolomite units at Meteor Crater do not appear to exhibit any colonization, regardless of the level of shock. The Coconino sandstone units, on the other hand, present distinct enhancements in microbial colonization, but only at low and moderate levels of shock, not at high shock levels. The complex trends at Meteor Crater are likely to be due to differences in target lithology, level of shock, and environmental factors such as the local weathering regime, consistent with the suggestion that these parameters play an important role in determining the viability of impact-induced lithic microbial habitats.

Such habitats are of interest to astrobiology in that they connect impacts, a fundamental and universal process of planetary formation and evolution, with life, particularly microbial life. Impacts are shown 
to be of positive influence in the development of microbial life and might have played a key role in the creation of viable (warm, moist and sheltered) habitats on early Earth and Mars.

\section{The function of most cyanobacterial signature genes at $\sim 2$. I Ga is unknown \\ Kirt Martin $\bullet$ University of Houston, Janet Siefert $\bullet$ Rice University, Yue Lu • University of Houston, Pedro Moreno $\bullet$ University of Houston, William Widger $\bullet$ University of Houston, George Fox $\bullet$ University of Houston}

Although claims for the earliest fossilized cyanobacteria at $3.5 \mathrm{Ga}$ have been questioned, there is strong agreement that biomarkers in wellpreserved sediments reveal the presence of cyanobacteria at $2.7 \mathrm{Ga}$. Oxygenic photosynthesis is a unique aspect of the cyanobacterial phenotype and would certainly have been the source for the rise of oxygen in the Archean atmosphere at $2.2 \mathrm{Ga}$. While the timing of this rise seems well established, the trigger for the event remains uncertain. The evolution of oxygen-producing photochemistry and associated metabolic or cellular innovation is integral to explaining this time lag. To this end the photosynthetic pathways have been studied in detail. However, the extent to which cyanobacteria may have had unusual biochemical properties and what this unknown biochemistry could have contributed to their Archean phenotype are unexplored. We report the results of genomic comparisons for six cyanobacteria. We found that at least 170 genes are conserved among these cyanobacteria, which are not generally associated with other bacterial genomes. Although a number of these cyanobacterial signature genes code for photosynthetic function, the role of the vast majority is unknown. Evidently, the unique aspects of the cyanobacterial phenotype are far less understood than one might have expected. It remains to be determined what other unique biochemistry the cyanobacteria may have possessed.

\section{Origin and evolution of photosynthesis \\ Lucas Mix $\bullet$ Harvard University, Tara Harmer $\bullet$ Harvard University, David Haig $\bullet$ Harvard University, Colleen Cavanaugh $\bullet$ Harvard University}

$99 \%$ of the energy budget for all life on Earth is ultimately supplied by one reaction. Photosynthesis occurs in five groups of bacteria, however the evolutionary relationships between them remain unclear. 16S rRNA phylogenies place groups throughout the Bacterial tree separated by non-photosynthetic taxa, yet similarities of protein structure and function make it improbable that photosynthesis arose more than once. Evolutionary models based on other genes have been called into question due to small sample sizes, conflicting results and the possibility of horizontal transfer. It is difficult to determine whether the results reflect the history of photosynthesis. We address this problem by looking at reaction center proteins that carry out photosynthesis. Relationships between three groups (Heliobacteria, Chlorobiaceae and Cyanobacteria) are elucidated by analysis of type one reaction centers (RC1), which have an $\mathrm{Fe}-\mathrm{S}$ cluster. A convincing $\mathrm{RC} 1$ phylogenetic has to overcome problems with alignment and limited sequence data. While whole sequences could not be aligned, a phylogeny of type-one reaction centers was constructed using functional domains. Work is currently proceeding to generate more reaction center sequence data from the Heliobacteria to eliminate long branches from the resulting tree.

\section{Variation of the nitrogen fixation rate by lightning during the evolution of the atmosphere} Rafael Navarro-Gonzalez $\bullet$ Universidad Nacional Autonoma de Mexico, Delphine Nna Mvondo $\bullet$ Universidad Nacional Autonoma de Mexico, Christopher Mckay $\bullet$ NASA Ames Research Center, Patrice Coll • Universites Paris, Francois Raulin • Universites Paris

The availability of reactive nitrogen species was a fundamental prerequisite for the emergence and sustainability of life on this planet. One of the principal reservoirs of nitrogen on the Earth has been the atmosphere where it is present in molecular form. Nitrogen is inert under normal atmospheric conditions; its conversion into reactive forms, a process referred to as nitrogen fixation, is highly endothermic. In this paper we report new experimental data on the variation of the nitrogen fixation rate by lightning during the evolution of the early Earth's atmosphere from predominantly carbon dioxide to predominantly molecular nitrogen and finally during the rise of atmospheric molecular oxygen. Lightning in the laboratory was simulated by a laserinduced plasma produced by focusing a high-power Nd-YAG laser inside a flask containing different mixtures of carbon dioxide in nitrogen and oxygen in nitrogen at various proportions (0-100\%). Nitric oxide was identified as the main $\mathrm{N}$-product by electron impact mass spectrometry and infrared spectroscopy.

\section{Nitrogen as a chemical and isotopic biomarker for Archean biochemistries}

Dominic Papineau $\bullet$ University of Colorado, Boulder, Stephen J.

Mojzsis • University of Colorado, Boulder

Knowledge of environmental conditions for the emergence of life on Earth and the nature of the earliest metabolic pathways are crucial in the search for extraterrestrial biochemistries. Phylogenetic analyses reveal that nitrogen-fixing organisms are in both the Archaeal and Bacterial domains and studies of nitrogen-fixing genes demonstrate that this may have evolved prior to the last common ancestor. Metabolically fixed nitrogen in macromolecules can be mineralized to $\mathrm{NH}_{4}^{+}$by degradation of organic matter and it is well known that $\mathrm{NH}_{4}^{+}$substitutes for $\mathrm{K}^{+}$during clay formation. Subsequent metamorphism of clays leads to the crystallization of micas, which readily incorporate $\mathrm{NH}_{4}^{+}$. We use mFTIR spectrometry to determine the $\mathrm{NH}_{4}^{+}$concentration of micas from early Archean metapelites. We recognize that by itself $\mathrm{NH}_{4}^{+}$ is a potential biomarker, but a more reliable interpretation requires measurements of nitrogen isotopic values. Biochemical evidence demonstrates that during nitrogen fixation $\mathrm{d} 15 \mathrm{~N}$ is slightly negatively fractionated with respect to the nitrogen source. Some data suggest that $\mathrm{d} 15 \mathrm{~N}$ values from Archean carbonaceous residues record $\mathrm{N}_{2}$ fixation. As a test of this hypothesis, we report preliminary $\mathrm{NH}_{4}^{+}$concentration measurements coupled with $\mathrm{d} 15 \mathrm{~N}$ values of biotites from a sequence of early Archean schists and evaluate potential biological sources for the $\mathrm{NH}_{4}^{+}$.

\section{A hierarchical model of the emergence of life both as a probabilistic and a deterministic conjecture} Radu Popa $\bullet$ University of Southern California Probing the essence of life independent of its physical nature is required for understanding early evolution, energy dissipative systems, artificial life and space exploration. A model of life should address: fundamental/ emergent properties, overall purpose and driving forces. A hierarchical model for the origin of life (energy, boundary, order, handedness and information) is presented in which probabilistic phase transitions increase the likeliness of subsequent events. Life's origin is both probabilistic and deterministic. Catalysis, reflexive activity, transduction, self-impaired exchange, order self-control, replication and cryptic information are fundamental features and collectively define life. Energy dissipation, growth, directional flow, homeostasy, metabolism, reproduction, motion, biomineralization, competition and evolution are but emergent properties. Fractionation, chirality, proteins, DNA, ${ }^{14} \mathrm{C}$ fixation or photosynthesis are not suitable as non-Earthcentric parameters. Life is: any strategy using internal information (error tolerant and cryptic), to build, to maintain and to adjust negentropic energy dissipative entities to space-time fluctuations. The natural force promoting life into existence is related to the second law of thermodynamics lowering its selective pressure on self-controlled energy dissipative entities.

\section{A diverse assemblage of fossil testate amoebae from the Neoproterozoic ( $742 \mathrm{Ma})$ Chuar Group, Grand Canyon: implications for early Eukaryotic ecology and evolution}

Susannah Porter $\bullet$ Harvard University, Ralf Meisterfeld $\bullet$ RheinischWestfalische Techn. Hochschule, Aachen, Germany, Andrew Knoll • Harvard University

An exceptionally preserved assemblage of vase-shaped microfossils 
(VSMs) from the $\sim 742 \pm 6$ Ma Chuar Group, Grand Canyon, provides morphological and taphonomic details that indicate affinities with the testate amoebae. Using modern testate amoeban taxonomy as a guide, we have identified 12 species of VSMs in the assemblage, ten of which are new. A few species have character combinations closely approximating those found in specific lobose and filose testate amoeban genera, suggesting that at least stem group, and possibly crown group, representatives of these taxa were present $742 \mathrm{Ma}$.

A few VSMs have semicircular holes in the side of their tests, probably reflecting the activities of a predator or scavenger. Thus by $742 \mathrm{Ma}$, complex food webs had emerged: bacteria were eating dead primary producers; protozoa were eating both primary producers and bacteria, and additional organisms were eating these protozoa.

Relationships among crown group eukaryotes are not resolved. A recent phylogeny suggests that lobose testate amoebae are part of an "Amoebozoa" clade that is sister to the animals + fungi. If correct, VSMs indicate that a direct ancestor of animals + fungi was present by $\sim 742 \mathrm{Ma}$.

\section{Fe isotope compositions of hot springs deposits, Chocolate Pots Hot Springs, Yellowstone National Park}

Rebecca L Poulson • University of Wisconsin, Madison, Brian L. Beard - University of Wisconsin, Madison, Beverly K. Pierson • University of Puget Sound, Clark M. Johnson • University of Wisconsin, Madison Chocolate Pots Hot Springs in Yellowstone, with its Fe(II)-rich $(\sim 0.1 \mathrm{mM})$ anoxic waters, provides a possible analog for ancient conditions on Earth or Mars, and bears on the problem of Fe cycling in oxygen-poor environments. As springs flow down to the Gibbon River, $\mathrm{Fe}$ is oxidized through contact with the atmosphere and with mats of photosynthetic bacteria. Oxidation of $\mathrm{Fe}(\mathrm{II})$ results in rapid precipitation of $\mathrm{Fe}$ oxyhydroxides. Along three downflow paths, water temperatures decrease from 52 to $45^{\circ} \mathrm{C}, \mathrm{pH}$ increases from 5.5 to 7 , and $\mathrm{d} 56 \mathrm{Fe}$ values for ferrihydrite decrease from +0.9 permil to -1.6 per mil. The $\mathrm{d} 56 \mathrm{Fe}$ values for aqueous $\mathrm{Fe}$ also generally decrease with distance; -0.7 per mil measured near the source, and -1.2 per mil at the base. Fractionation between aqueous $\mathrm{Fe}$ and ferrihydrite is large $(+1.6$ per mil) near the vent, decreases, and even changes sign with distance $(-0.4$ per mil at the base). Fe isotope fractionation between ferrihydrite and $\mathrm{Fe}$ (II) is positively correlated with water temperature, and inversely correlated with $\mathrm{pH}$. Variations in Fe isotope fractionation may be attributed to changes in Fe speciation, kinetic effects associated with the rapid precipitation of $\mathrm{Fe}$ oxyhydroxides, and/or biological oxidation of $\mathrm{Fe}$, and these observations illustrate the great potential that $\mathrm{Fe}$ isotope geochemistry has for tracing ancient geochemical cycling of $\mathrm{Fe}$.

\section{Comparing standard Darwinism and strong panspermia: a bioinformatic analysis of human, mouse and intervening genomes}

Thomas Ray $\bullet$ University of Oklahoma, Brig Klyce • Acorn Enterprises $L L C$

Standard Darwinian theory holds that new genetic programs arise from existing ones through gene duplication and divergence. Strong panspermia holds that new genetic programs are imported to the Earth's biosphere and installed by gene transfer. The objective of the current research is to use bioinformatic analysis of genomic data to compare the two theories.

The primary target will be the origin and evolution of gene families. A high proportion of new gene families arising by gene duplication and divergence is consistent with standard Darwinian theory. A high proportion arriving by horizontal transfer is consistent with strong panspermia.

The study will begin with a comparison of the human and mouse genomes, to identify gene families present in humans, but absent in mice. Then we will seek these candidate gene families in every species between human and mouse for which BACs are available. As completed genomes for primates and other intervening species become available these databases will also be searched. We believe that sufficient data will be available to produce a clear result within 3 years.

In the first year, we plan to develop analytical protocols for:

(a) Defining and recognizing "gene families."

(b) Comparing human and mouse genomes to identify "new gene families."

(c) Seeking these new gene families in intermediate genomes.

(d) Discriminating between duplication/divergence and horizontal transfer.

\section{Whole genome analyses of photosynthetic bacteria and the evolution of photosynthesis}

Jason R. Raymond • Arizona State University, Olga Zhaxybayeva $\bullet$ University of Connecticut, J. Peter Gogarten $\bullet$ University of

Connecticut, Robert E. Blankenship $\bullet$ Arizona State University

Information contained within individual sequences provides an insight into the evolution of specific genes or biochemical pathways, while the evolutionary history of an organism is recorded within its genome. We have done a comparative analysis of genomes from four distinct families of photosynthetic bacteria using classical phylogenetic techniques as well as newer methods that circumvent some of the limitations of sequence analysis. The taxa analyzed include representatives from cyanobacteria, green sulfur, green non-sulfur and purple bacteria. Some phylogenetic signatures are consistent with evolution through vertical descent, although a surprising number of orthologs have complex evolutionary histories that infer frequent lateral transfers.

We have also differentially compared genomes from photosynthetic and non-photosynthetic organisms in order to identify genes specific to photosynthesis. While only a small subset of photosynthesis-specific genes were identified, remarkably nearly half of these genes are either hypothetical or completely uncharacterized, suggesting some essential elements of the photosynthetic apparatus remain to be identified. The algorithms we are developing can be easily tuned to find genes specific to other pathways, for example nitrogen fixation or sulfate reduction, thus providing roads into biochemical inquiry based on in silico applications.

\section{Complex life in a complex ecosystem: morphological diversification within thermal spring habitats}

Peter Roopnarine $\bullet$ California Academy of Sciences, Carol Tang • California Academy of Sciences

Cuatro Cienegas, Mexico, hosts a series of isolated thermal spring systems comprising trophically and taxonomically complex ecosystems of bacterial and eukaryotic producers, and metazoan grazers and predators. As such, this system serves as a natural evolutionary laboratory and ecological microcosm in which to study biotic interactions and processes of diversification. The aim of the present study is to quantify levels of diversification of an endemic snail fauna that represents one of the major components of the metazoan consumer trophic level. The study is part of an integrated investigation of the chemical, biological, and physical aspects of these ecosystems that will allow for a more complete understanding of their evolutionary and ecological dynamics.

Quantitative landmark-based morphometric analysis of the snail genus Mexipyrgus indicates an unusually high level of morphological diversification within this limited geographic area. Eight sampling localities from five separate hydrological systems each yield significantly different morphologies. Samples are distinct both between and within spring systems but follow predictable geographic patterns; for example, in the Churince and Mojarral systems, differentiation is correlated with downstream position from the springhead.

\section{The indirect effect of UV: some good news for microbes?}

Lynn Rothschild $\bullet$ NASA Ames Research Center, Diane Purcell $\bullet$ SETI Institute, Dana Rogoff $\bullet$ SETI Institute, Cindy Wilson $\bullet$ University of Montana

Ultraviolet (UV) radiation is of great concern because its biological effects are predominantly harmful. UV damage may be direct or indirect, 
the latter mediated through the photochemical production of reactive oxygen species such as hydrogen peroxide. We measured the effect of $\mathrm{H}_{2} \mathrm{O}_{2}$ on various microbes both in the laboratory and in nature. At our study site in Yellowstone National Park, there is a UV-induced diurnal fluctuation of $\mathrm{H}_{2} \mathrm{O}_{2}$ extending up to $1 \mu \mathrm{m}$ molar. Levels of DNA synthesis resulting from exposure to $\mathrm{H}_{2} \mathrm{O}_{2}$ were measured in several algal mats. Within naturally occurring concentrations of $\mathrm{H}_{2} \mathrm{O}_{2}$, DNA synthesis increased. Laboratory studies showed that similar concentrations of $\mathrm{H}_{2} \mathrm{O}_{2}$ induce mitosis. We hypothesize that the low levels of $\mathrm{H}_{2} \mathrm{O}_{2}$ encountered in nature are inducing mitotic division. At higher levels of $\mathrm{H}_{2} \mathrm{O}_{2}$ a second peak in DNA synthesis was found which we interpret to represent DNA damage repair. These experiments suggest that in nature, the low levels of $\mathrm{H}_{2} \mathrm{O} \mathrm{H}_{2} \mathrm{O}_{2}$ produced may have a mitogenic rather than a damaging effect. Assuming early levels of UV radiation were substantially higher at the time protists evolved, differential mitogenic effects could have influenced microbial evolution. With $\mathrm{H}_{2} \mathrm{O}_{2}$ likely to be present on such bodies as Mars and Europa, the response of organisms will be concentration-dependent and not linear.

\section{Analysis of the influence of cosmic and geophysical factors on influenza viruses reproduction}

Michael I. Ryabov $\bullet$ National Academy of Sciences, Odesa, Ukraine, Alla S. Fedchuk • National Academy of Sciences, Odesa, Ukraine, Victor P. Lozitsky $\bullet$ National Academy of Sciences, Odesa, Ukraine, I.I. Mechnikov •Ukrainian Research Anti-Plague Institute, Odesa, Ukraine; Odesa National University, Ukraine, Oleksandr P. Fedchuk • Odesa National University, Ukrane

The regular annual periodicities in various viral epidemics have been registered at the world-wide scale. The factors regulating the intensity of epidemics and their emerging rate were numerous, including individual features of virus-cell interaction, density of population and state of environmental ambience.

We have studied influenza virus reproduction with a model of tissue culture under the influence of solar and geomagnetic activity. It was discovered at a statistically reliable level that the harvest of viral generations was quantitatively different under equal doses of infectious agent, [A Hong Kong 1/68(H3N2)] and the standard conditions of laboratory experiments as well.

We have conducted a comparative analysis of the state of solar and geomagnetic activity during the dates of the virus reproduction experiments. We proposed to discuss instead of traditionally used data such as Wolf numbers and the value of geomagnetic indices, to take into account the entire set of solar and geophysical factors influencing the state of the Earth's biosphere now obtainable through a network of terrestrial and cosmic stations. As a result we have obtained stable correlations between the increase or decrease of the viral harvest and factors such as the geomagnetic field state index, the number of solar explosions, the flux of the UV part of solar radiation and the data characterizing the fluxes of solar particles and cosmic rays. The proposed approach could be used in the field of prognostics of viral epidemics and the tactics of their prophylactics as well.

\section{Rates of biologically significant cosmic explosions \\ John Scalo • University of Texas, Austin, J. Craig Wheeler •} University of Texas, Austin, Peter Williams $\bullet$ University of Florida Planets are stochastically subjected to large ultraviolet (UV) and ionizing fluxes from astronomical explosions and flares that can affect atmospheric chemistry and biological activity through direct mutational enhancement or sterilization. Assuming mutational thresholds for UV and gamma-ray fluences for eukaryotic-like organisms, we have estimated the typical rate of occurrence of biologically significant supernovae, gamma-ray bursts, superflares, and novae. Supernovae emit about $10^{47} \mathrm{ergs}$ of UV in the shock breakout and later phases. Accounting for the Galactic spatial distribution of supernovae and UV extinction by dust, we calculate the cumulative probability per unit time of supernova UV fluences and fluxes. The rate of significant supernova UV " jolts" that beat parent star backgrounds is about 1000 per Gyr for satellites of gas giants more than about $5 \mathrm{AU}$ from solar-type stars or for planets in the habitable zones of K or M stars. Supernovae produce as much as $10^{49}$ ergs of gamma-ray emission associated with radioactive decay and yield chemically and biologically interesting doses at a similar rate. We estimate a biologically significant gamma-ray burst rate of 100-500 per Gyr. The effects of the gamma-ray sources depend on gamma- and X-ray redistribution in the planetary atmosphere. For prokaryotic organisms, the estimated rates are 10-100 times smaller.

\section{Parallel genome assembly: a new model for explaining the origin and diversity of life on Earth} Periannan (Sena) Senapathy $\bullet$ Genome Technologies

The theory of evolution assumes that a bacterial-like cell originated from inanimate matter, which then evolved into all other organisms, including multicellular animals and plants, through biological evolution - a linear, branching tree-like, descent of organisms with modification. This theory has had many problems in explaining the morphological and fossil data, and most recently, the modern genome data. A new theory based on the direct abundant occurrence of eukaryotic genes - not prokaryotic genes - in the primordial pond, states that the genomes of numerous distinct eukaryotic organisms, including those of multicellular animals and plants, were assembled in parallel from the common pool of genes in a primordial pond. This theory clearly explains all three types of data unexplained by the conventional theory of evolution - the modern genome data, morphological distinction of organisms and the fossil record of the Cambrian Explosion. This model also shows that it is very highly probable for life, in fact multicellular life, to exist on any planet with conducive physical and chemical conditions in any star system in the Universe. Therefore, life may exist in abundance in the Universe.

\section{Sources of hydrogen and $\mathrm{H}$-isotopic fractionations in bacterial lipid biosynthesis}

Alex Sessions $\bullet$ WHOI, Linda Jahnke $\bullet$ NASA Ames Research Center, Arndt Schimmelmann $\bullet$ Indiana University, John Hayes $\bullet$ WHOI

The $\mathrm{D} / \mathrm{H}$ ratios of bacterial lipids will be controlled by the sources of $\mathrm{H}$ and by isotopic fractionations on the pathways between those sources and lipid products. Seeking the simplest possible heterotrophic system, we have examined Methylococcus capsulatus, which obtains energy and biomass from the aerobic oxidation of methane. This organism can obtain $\mathrm{H}$ only from one of the four equivalent positions on $\mathrm{CH}_{4}$ or one of the two equivalent positions on $\mathrm{H}_{2} \mathrm{O}$. We systematically varied isotopic compositions of available methane and water and measured isotopic compositions of individual fatty acids, sterols, and hopanols from six cultures. Our results show that $31 \pm 2 \%$ of the $\mathrm{H}$ in all the lipids analyzed, independent of structure, comes from methane and the rest from water. All $\mathrm{H}$ appears to flow through the intracellular-water pool. A balance of diffusional processes is plausibly responsible for regulating the fraction of cellular water derived from the oxidation of methane. Fractionations are small in comparison with those observed in plants. Relative to water, depletions in D are $50 \%$ for fatty acids and $150 \%$ for isoprenoids. Depletions relative to methane are 60 and $210 \%$ for fatty acids and isoprenoids, respectively. The results and difficulties in their interpretation point to key questions for future investigations

\section{Retortamonad flagellates are closely related to diplomonads implications for the history of mitochondrial function in eukaryote evolution} Jeffrey Silberman $\bullet$ University of California, Los Angeles, Alastair Simpson • Dalhousie University, Nova Scotia, Canada, Jaroslav Kulda - Charles University, Czech Republic, Patricia Johnson • University of California, Los Angeles, Andrew Roger $\bullet$ Dalhousie University, Nova Scotia, Canada.

The "archaezoa hypothesis," which posits that certain living unicellular eukaryotes lacking mitochondria diverged prior to acquisition of the mitochondrial symbiont, is incompatible with some of the cell-fusion models for the origin of eukaryotes. Emerging molecular data have shrunk the pool of lineages proposed never to have experienced the symbiosis leading to the mitochondrion. We present a molecular phylogenetic examination of the evolutionary position of retortamonads, a group of mitochondrion-lacking flagellates, and the 
last plausible candidate "archezoan." Our phylogenies of both small subunit ribosomal RNA and heat-shock protein 90 sequence data demonstrate a robust phylogenetic association of the retortamonads with another amitochondriate group, the diplomonads, indicating that they share an amitochondriate common ancestor. Since diplomonads appear to retain genes of mitochondrial origin, they have probably lost their mitochondria secondarily (rather than being ancestrally amitochondriate). By virtue of their relationship to diplomonads, our results imply that retortamonads are also secondarily amitochondriate. Of the various groups of eukaryotes originally suggested to be primitively amitochondriate under the "archezoa hypothesis," all have now been found to have physical and/or genetic mitochondrial relics, or form a robust clade with an organism with such a relic.

\section{An ancient microbial ecosystem: Precambrian analog} of life in the cosmos

Leigh Anne Smith $\bullet$ University of California, Los Angeles, J. William Schopf $\bullet$ University of California, Los Angeles

Since its inception, Astrobiology has been intertwined with the field of Precambrian paleontology. The microbe-dominated early Earth represents our best opportunity for beginning to understand the requirements of life outside our world.

The study presented here focuses on the $\sim 850$-Ma-old microbial community petrified in carbon-rich cherts of the Bambui Group of Brazil. This formation has been described as "the best preserved and most diverse Precambrian microbiota known from South America" [Important because it] includes the most varied assemblage of fossil filamentous bacteria now known from the geologic record, worldwide (Fairchild et al. 1996, Precambrian Res. 80, 125-152).

On the basis of morphometric studies by optical microscopy, molecular analyses by laser-Raman spectroscopy, and elemental and isotopic measurements by ion microprobe mass spectrometry of these fossil bacteria and their modern analogs, our goal is to define their physiological capabilities and the flow of carbon and energy (fuel and oxidants) within their fossil ecosystem. Understanding the metabolic characteristics of these life forms will help us reconstruct the ecology and evolution of early Earth and may eventually lead to a greater understanding of such systems on other worlds.

\section{The evolutionary relationship between nitrogen fixation and bacteriochlorophyll synthesis}

Christopher Staples $\bullet$ Arizona State University, Yuichi Fujita $\bullet$ Osaka University, Carl Bauer •Indiana University, Robert Blankenship • Arizona State University

Photosynthesis developed relatively soon after the origin of life on Earth. Some reports of stromatolites suggest that this development occurred as early as 3.5 Gya, while the meteorite bombardment period ended 3.9 Gya. However, due to the complexity of photosynthesis, it is highly likely that the earliest life forms were not photosynthetic. It is thus proposed that photosynthesis developed as a favorable branch of a pre-existing, more fundamental pathway. From phylogenetic analyses and emerging biochemical data, it is proposed that this pre-existing pathway was that currently utilized for nitrogen fixation (the Nif proteins). It is currently unclear whether the Nif proteins were originally utilized in nitrogen fixation (in the case of a neutral atmosphere), or hydrocyanic acid detoxyase (in the case of a reducing atmosphere). In this poster, proposals for the study of the genetic, structural, functional, and mechanistic relationships between selected proteins in the anaerobic bacteriochlorophyll synthesis (bch) pathway and the nitrogen fixation (nif) pathway are presented (BchNB, BchYZ with NifDK and NifNE; $\mathrm{BchL}$ and $\mathrm{BchX}$ with $\mathrm{NifH})$ and preliminary results are shown.

\section{The evolutionary diversification of cyanobacteria: molecular-phylogenetic and paleontological perspectives}

Akiko Tomitani $\bullet$ Kyoto University, Andrew Knoll $\bullet$ Harvard University

Cyanobacteria have played a significant role in Earth's history as primary producers and the ultimate sources of atmospheric oxygen. An understanding of their evolution provides a key to the elucidation of early biological and environmental history. In tandem, molecular phylogeny and paleontology provide constraints on the pattern and timing of cyanobacterial diversification.

$16 \mathrm{~S}$ rRNA, rbcL and hetR genes were newly isolated from 21 filamentous cyanobacteria distributed among 16 genera. Phylogenetic trees inferred from the gene markers support the hypothesis that cyanobacteria capable of cell differentiation (heterocysts and akinetes) form a monophyletic clade among undifferentiated filaments and coccoid forms.

Akinete fossils are common in $c a$. $1500 \mathrm{Ma}$ cherts from tidal flat carbonates of the Billyakh Group, Siberia. Rare akinetes have also been found in silicified carbonates of the $c a$. 1650 Ma Amelia Dolomite, northern Australia, with other microfossils representing cyanobacterial diversity. What may be the earliest known akinetes are preserved in $2100 \mathrm{Ma}$ Francevillian cherts from West Africa. Given geochemical evidence suggesting that oxygen first reached levels that would compromise nitrogen-fixation (and hence select for heterocyst differentiation) 2400-2200 Ma, the geological record may provide both upper and lower bounds on the radiation of heterocystous cyanobacteria.

\section{The origin of red algae, monophyly of plastids, and Precambrian calibration of molecular clocks Maria Venturi • Pennsylvania State University, S. Blair Hedges •} Pennsylvania State University

Much of the early history of life remains unclear, in part because of the relatively poor Precambrian fossil record. Although sequence data are rapidly becoming available for time estimation of early events in evolution, there are no robust fossil calibration points in the Precambrian. Here we analyze sequence data sets of many nuclear proteins to investigate whether red algae, dating to 1200 million years ago (Ma), represent an exception. We find strong support for red algae being on the plant lineage, indicating a single origin for plastids, and estimate that red algae diverged from plants only slightly earlier than the earliest fossil red algae. Although this suggests that the red algae/plant split is perhaps the best Precambrian fossil calibration, it is nonetheless a minimum estimate of divergence time. The best calibrations for timing early events continue to be Precambrian divergence times estimated from other molecular clock studies using vertebrate calibrations.

\section{Isolation of genomic DNA from carbonate-encrusted, stromatolitic communities for molecular analyses} Brian D. Wade • Arizona State University, Ferran Garcia-Pichel • Arizona State University

Carbonate-encrusted, stromatolitic communities have been studied previously using traditional methods, but no studies exist that use molecular techniques for this purpose. However, molecular techniques are not designed for rock-like samples. We evaluated three methods for isolating genomic DNA from such samples with the aim of optimizing their characterization. A single carbonate-encrusted, stromatolitic, cyanobacteria-dominated oncoid was used as a representative sample. The first method involved dissolution of the carbonate in a $\mathrm{HCl}$ solution and subsequent homogenization before isolating the DNA using phenolchloroform. Pulverization of the sample without carbonate dissolution before DNA isolation was employed in the second method. The third method used ethylenediaminetetraacetic acid (EDTA) to dissolve the carbonate before DNA isolation. $\mathrm{HCl}$ dissolution yielded no DNA. Isolated DNA from successful methods was PCR-amplified using cyanobacteria-specific primers. PCR products were separated via denaturing gradient gel electrophoresis (DGGE) to provide cyanobacterial community fingerprints of the oncoid. Comparison of the fingerprints showed that pulverization without carbonate dissolution and EDTA dissolution before DNA isolation give identical fingerprints of the cyanobacterial community. EDTA dissolution resulted in intact samples, allowing spatial differentiation. 
Equilibrium fractionation between aqueous ferrous and ferric iron

Susan Welch • University of Wisconsin, Madison, Brian Beard • University of Wisconsin, Madison, Clark Johnson • University of Wisconsin, Madison

In order to understand the large variability observed for $\delta 56 \mathrm{Fe} / 54 \mathrm{Fe}$ ratios in solution and in chemical precipitates such as banded iron formations, we have conducted a series of experiments to determine the factors affecting equilibrium fractionation between aqueous ferrous and ferric iron over a range of $\mathrm{Fe}(\mathrm{II}): \mathrm{Fe}(\mathrm{III})$ ratios, $\mathrm{Cl}^{-}$concentrations, and temperature.

The $\mathrm{Fe}(\mathrm{III})-\mathrm{Fe}(\mathrm{II})$ fractionation determined for hexa-aquo $\mathrm{Fe}$ in $\mathrm{pH}$ 2.2 nitrate solutions at room temperature, $\sim 22{ }^{\circ} \mathrm{C}$, is $2.76 \pm 0.1$ per mil. Addition of up to $100 \mathrm{mM} \mathrm{Cl}^{-}$as $\mathrm{LiCl}(5 \%$ ferrous and $42 \%$ ferric chloro complexes in solution) had no significant effect on the $\mathrm{Fe}(\mathrm{III})$ $\mathrm{Fe}$ (II) fractionation of $\sim 2.76 \pm 0.06$ per mil. At $5{ }^{\circ} \mathrm{C}$, the $\mathrm{Fe}$ (III)-Fe(II) fractionation was $\sim 0.8$ per mil higher.

These measured isotope fractionations are about half the values predicted from spectroscopic data. These probably represent the maximum values since partial re-equilibration between the ferrous and ferric species during separation will yield lower measured fractionations. These results indicate that changes in the inorganic speciation of ferrous and ferric iron over a range in chloride concentration from fresh water to seawater should have little effect on the equilibrium fraction. However, other factors, such as changes in temperature or non-equilibrium processes could dramatically change the ratio $\delta 56 \mathrm{Fe} / 54 \mathrm{Fe}$.

\section{Endemic archaeal populations from extreme environments: biogeography and molecular} evolution of the hyperthermophilic crenarchaea, Sulfolobus "islandicus"

Rachel Whitaker $\bullet$ University of California, Berkeley, Dennis Grogan • University of Cincinnati, John Taylor $\bullet$ University of California, Berkeley

What are the evolutionary forces that promote or constrain diversification in a microbial species? We explore this question in a species of hyperthermophilic archaea called Sulfolobus "islandicus" with a particular interest in how life evolves under the unique constraints of an extreme environment. We use multilocus sequence typing of 12 independent, polymorphic housekeeping genes in population genetic analyses to show that there are isolated, endemic populations within the species $S$. "islandicus." This work shows that the natural history of S. "islandicus" has been strongly influenced by a microevolutionary force that has largely been ignored in microbial evolution - isolation by distance. In addition, we examine the patterns of nucleotide substitutions in protein coding genes between Sulfolobus species for evidence of selection imposed on organisms living at the extremes of temperature and acidity. We analyze associations of alleles sampled from around the genome for evidence of genetic exchange within natural archaeal populations. Our investigation of the geographic distribution, intensity of selection and levels of recombination in $S$. "islandicus" is the first to comprehensively explore the effects of classic microevolutionary forces on microbial evolution in an extreme environment.

\section{The nitrogen biogeochemical cycles in the Archean inferred from shale geochemistry}

Kosei Yamaguchi • Pennsylvania State University, Hiroshi Naraoka • Tokyo Metropolitan University, Hiroshi Ohmoto $\bullet$ Pennsylvania State University

The present-day marine nitrogen cycle is characterized by a series of oxidation-reduction reactions (e.g. $\mathrm{N}_{2}$-fixation, nitrification, and denitrification). These reactions are mediated by a variety of organisms. An important question is the timing of the onset of such processes of nitrogen redox cycling in the Earth's history. For this purpose, we conducted a geochemical study of 3.25-2.22 billion-year old carbonaceous sedimentary rocks ( $>60$ samples) from South Africa and Australia, focusing on their stable isotopic compositions. The characteristics of nitrogen isotopic compositions of organic-bound nitrogen and clay-bound nitrogen in those rocks are essentially the same as those of the Cretaceous black shales and modern marine sediments. These data suggest that the present day-styled, microbially mediated, nitratebased redox-cycling of nitrogen has already operated in the oceans since at least 3.25 billion years ago. This study has important implications for the redox evolution of atmosphere and oceans, and for the evolution of the biosphere.

\section{Bootstrap, Bayesian probability and maximum likelihood mapping: exploring new tools for comparative genome analyses}

Olga Zhaxybayeva $\bullet$ University of Connecticut, Lorraine Olendzenski $\bullet$ University of Connecticut, J. Peter Gogarten • University of Connecticut

Horizontal gene transfer (HGT) played an important role in shaping microbial genomes. In addition to genes under sporadic selection, HGT also affects housekeeping genes and those involved in information processing, even ribosomal RNA encoding genes. Here we describe tools that provide an assessment and graphic illustration of the mosaic nature of microbial genomes.

We adapted maximum likelihood (ML) mapping to the analyses of all detected quartets of orthologous genes found in four genomes. We have automated the assembly and analyses of these quartets of orthologs given the selection of four genomes. We compared the ML-mapping approach to more rigorous Bayesian probability and bootstrap mapping techniques. The latter two approaches appear to be more conservative than ML-mapping, but qualitatively all three approaches give equivalent results. All three tools were tested on mitochondrial genomes, which presumably were inherited as a single linkage group.

We applied these tools to study interphylum and interdomain relationships. While some type of genes were found to be less prone to interdomain HGT than others, the performed analyses demonstrate that relationships among prokaryotes cannot be accurately depicted by the tree-like evolution of a core of rarely transferred genes; rather prokaryotic genomes are mosaics in which different parts have different evolutionary histories

\section{Extreme environments}

\section{Probing the limits of anaerobic bioenergetics: experimental and modeling studies of secondary fermentations \\ Cameron J. Adams $\bullet$ University of California, San Diego, David L. Valentine $\bullet$ Scripps Institution of Oceanography; University of California, San Diego; University of California, Santa Barbara}

Anaerobic microbial metabolism serves as a possible analog to life on early Earth as well as life elsewhere. Anaerobes persist in a variety of extreme environments and are adapted for a minimal energy lifestyle. Here we explore constraints on the bioenergetics of microbial growth by quantifying the minimum Gibb's free energy yields $\left(\Delta G^{\prime}\right)$ during growth of several "syntrophic" bacteria including Pelobacter acetylenicus (grown on ethanol), Syntrophobotulus glycolicus (grown on glycolate), Syntrophothermus lipocalidus (grown on butyrate), and Aminobacterium colombiense (grown on alanine). These organisms were grown as pure cultures using an $\mathrm{H}_{2}$-sparging vessel (Valentine et al. 2000, JMM, 39, 243-251), effectively decoupling the natural syntrophic associations of $\mathrm{H}_{2}$ producers and $\mathrm{H}_{2}$ consumers. Hydrogen concentration, $\mathrm{pH}$ and aqueous metabolite concentrations were monitored throughout each experiment in order to calculate the $\Delta G^{\prime}$ of catabolism. Each organism exhibited a distinct $\Delta G^{\prime}$ ranging from -26 to 
$-14 \mathrm{~kJ} \mathrm{~mol}^{-1}$ of substrate. The rate of metabolism was influenced by temperature and metabolite concentrations in a manner consistent with thermodynamic control (i.e. a constant $\Delta G^{\prime}$ was maintained independent of external changes). Kinetic modeling studies further indicate that the $\Delta G^{\prime}$ available to each organism is lower than previously calculated. These results provide a framework for considering the minimal energy requirements for growth of microbes elsewhere in the Universe.

\section{The relevance of heat and cold shock in Deinococcus radiodurans for space travel}

Alessandro Airo $\bullet$ Free University Berlin, Institute for Biochemistry, Susanne Chan $\bullet$ NASA Ames Research Center, Zak Martinez $\bullet$ University of California, Santa Cruz, Manu O. Platt • Georgia Tech; Emory University, Amber Sanford $\bullet$ NASA Ames Research Center, Johnathan D. Trent $\bullet$ NASA Ames Research Center

Heat shock and cold shock refer to molecular adaptations that enhance the survival of organisms at lethal high and low temperatures. We investigated the heat and cold shock response of the polyextremophile $D$. radiodurans to address issues associated with the conditions needed for panspermia as well as survival on a cold planet like Mars. We determined the optimal cold and heat shock temperatures and measured acquired cryo- and thermo-tolerance. A cold shock at $20^{\circ} \mathrm{C}$ for 3 hours significantly enhanced resistance to repeated freezing and thawing treatment. A heat shock at $42^{\circ} \mathrm{C}$ for 3 hours significantly enhanced survival at $52{ }^{\circ} \mathrm{C}$. Two-dimensional gel electrophoresis revealed an over expression of 42 proteins during cold shock and 67 proteins during heat shock. Among the heat-induced proteins are DnaK, GroEL, HSP20, and Superoxide dismutase, identified by MALDI-MS. Heat shock associated increases in mRNAs for HSP20, GroEL, GroES, DnaK, and GrpE were also observed by northern analysis. These results further our understanding of the molecular mechanisms for enhanced heat and cold tolerances, which is an essential area of research in astrobiology.

\section{Biogeochemical contrasts between subtidal and intertidal-to-supratidal hypersaline cyanobacterial} mats

Dan Albert $\bullet$ University of North Carolina, Chapel, Hill, Brad Bebout $\bullet$ NASA Ames Research Center, Mykell Discipulo $\bullet$ SETI Institute, Tori Hoehler $\bullet$ NASA Ames Research Center, Kendra Turk $\bullet$ University of California, Santa Cruz

Because the $>2.4 \mathrm{Gyr}$ fossil record of marine cyanobacterial mats is valuable for historical studies of evolution, we studied their descendants at Guerrero Negro, BCS, Mexico, specifically those that are dominated by Microcoleus (subtidal) and Lyngbya (intertidal to supratidal) cyanobacteria. We observed the exchange of $\mathrm{O}_{2}$ and dissolved inorganic $\mathrm{C}$ (DIC) between mats and the overlying water, during diel (24 hr) cycles. Microcoleus mats assimilated near-equal amounts of DIC during the day as they released at night, but Lyngbya mats typically showed net uptake of DIC over the diel cycle. Patterns of $\mathrm{O}_{2}$ daytime release and nighttime uptake mirrored these DIC trends in both mat types. Nighttime DIC effluxes from Microcoleus mats were equivalent in the presence versus absence of $\mathrm{O}_{2}$, whereas nighttime DIC effluxes from Lyngbya mats dropped markedly in the absence of $\mathrm{O}_{2}$. Thus, aerobic diagenesis was more important in Lyngbya mats than in Microcoleus mats, perhaps because trapped $\mathrm{O}_{2}$ bubbles persist only in Lyngbya mats at night and thus sustain populations of aerobes. In both mat types, effluxes of $\mathrm{H}_{2}$, $\mathrm{CH}_{4}$ and short-chain fatty acids were much greater at night in the absence of $\mathrm{O}_{2}$, emphasizing the importance of fermentation. Differences observed between Microcoleus versus Lyngbya mats forecast differences in their microbiota and in their patterns of gene expression.

\section{Characterization of bacteria isolated from briny water lenses in Siberian Permafrost \\ Corien Bakermans $\bullet$ NASA Jet Propulsion Laboratory, Alexander Tsapin $\bullet$ NASA Jet Propulsion Laboratory, Kenneth Nealson • University of Southern California}

Aerobic, heterotrophic bacteria were isolated from briny water lenses in
Siberian permafrost. These water lenses form due to salting out of the soil as the in situ temperature drops and remains at about $-10{ }^{\circ} \mathrm{C}$. Samples ranged in age from 30000 to 40000 years old and salinity was $120 \mathrm{~g} / \mathrm{l}$. Seventeen organisms were isolated by direct plating on $\mathrm{R} 2 \mathrm{~A} \pm 3 \% \mathrm{NaCl}$ at $4,10.5$, or $22{ }^{\circ} \mathrm{C}$ and represented the following genera: Psychrobacter, Arthrobacter, Frigoribacterium, Paenibacillus, Bacillus, Subtercola, Microbacterium, Rhodococcus, and Erwinia. Isolates were psychrotolerant (optimum growth temperature $=24$ $33{ }^{\circ} \mathrm{C}$ ); while halotolerance ranged from 0 to $10 \% \mathrm{NaCl}$. Rate of resazurin reduction was used to measure metabolic activity and correlated well with growth rate in the presence of $3 \% \mathrm{NaCl}\left(r^{2}=0.89-0.99\right)$. Spore-forming bacteria did not reduce resazurin at $-10{ }^{\circ} \mathrm{C}$. When detected, resazurin reduction at $-10{ }^{\circ} \mathrm{C}$ occurred at very low rates ( $0.6 \mathrm{fmol} / \mathrm{cell} /$ day); except the Psychrobacter sp. (6 fmol/cell/day). In addition, only the Psychrobacter $s p$. had a detectable growth rate at $-10{ }^{\circ} \mathrm{C}(0.02 \mathrm{l} /$ day $)$. These data suggest that at $-10{ }^{\circ} \mathrm{C}$ spore-forming isolates survived as spores, non-spore-forming isolates survived by maintaining metabolic activity sufficient only for cell repair, and the Psychrobacter $s p$. survived by maintaining metabolic activity sufficient for cell repair and reproduction.

\section{An endolythic microbial ecosystem at the Pacific coastal range of the Atacama Desert, Chile \\ Gomez-Silva Benito $\bullet$ Universidad de Antofagasta, Chile, Hector Olivares $\bullet$ Universidad de Antofagasta, Chile, Luis Caceres $\bullet$ Universidad de Antofagasta, Chile, Jorge Araya $\bullet$ Universidad de Antofagasta, Chile, Fred A. Rainey $\bullet$ Luisiana State University}

The Atacama Desert (ATD) in northern Chile is one of the driest and oldest hot deserts on Earth and represents a field laboratory to study life at its limits and a model for exobiological research. The barren dry hills and mountains of the Pacific Coastal Range of ATD, poorly explored from a microbiological perspective, are a topographic barrier to moisture-rich marine air. We have evidence to indicate that fissures and the soil-stone interface of translucent rocks in this area provide the microenvironmental niche for two desiccation-tolerant microorganisms, the cyanobacteria Chroococcidiopsis sp. and Streptomyces sp. In order to characterize the ecology and biology of this hypolithic community subjected to water and ultraviolet light stress, our research program includes the gathering of metereological data from the growth sites (temperature and relative humidity), isolation of the community members, TEM and SEM microphotographs, 16S rRNA gene sequence data, chemical composition of the rock substrate, absorption spectra from whole cell and cell extracts and to address the question of moisture availability from atmospheric water.

\section{Microbial processing of sulfur gases from a terrestrial cave ecosystem}

Philip Bennett • University of Texas, Austin, Annette Engel $\bullet$ University of Texas, Austin, Libby Stern $\bullet$ University of Texas, Austin The sulfur cycle is one of the basic chemical indicators of the presence of life on Earth. The marine sulfur system is extremely complex, where in addition to recycling of inorganic sulfur compounds, marine biota also produce a variety of volatile organic sulfur compounds (VOSC). In contrast, the continental surface and subsurface sulfur cycle is widely regarded as "simple," with only transformations between sulfate and sulfide considered significant. However, we find unexpected complexity in the sulfur cycle in a chemoautotrophically-based cave ecosystem. Dissolved sulfide provides the energy source to aquatic primary-producing sulfur-oxidizing bacteria, which in turn supports anaerobic microorganisms including fermenters and sulfate-reducing bacteria. In culture, these anaerobes produce $\mathrm{H}_{2} \mathrm{~S}$, as well as COS, DMS, and methanethiol, which may serve as important energy substrates for sulfur-oxidizers or methanogens within aquatic microbial habitats, or for subaerial cave-wall biofilms due to degassing into the cave atmosphere. Biogenic VOSCs are important to the global sulfur cycle, and may be indicators of microbial activity in subsurface environments. Moreover, as an extreme environment, the cave lacks photosynthetically 
fixed carbon, has toxic gases and locally extreme acidity, and extends our understanding of the limits of life.

\section{Chemical and isotopic signatures of microbial extraction of metal micronutrients from silicates S.L. Brantley • Pennsylvania State University, L. Liermann • Pennsylvania State University, J. Barling $\bullet$ University of Rochester, Rochester NY, A. Anbar • University of Rochester, Rochester NY}

$\mathrm{Fe}, \mathrm{Ni}$, and $\mathrm{Mo}$ are used by bacteria for enzymes, coenzymes, and cofactors. To extract $\mathrm{Fe}$, bacteria secrete siderophores; however, such high-affinity complexation mechanisms have generally not been documented for other metals. We have synthesized Fe-Ni-Mo-silicate glasses for incubation with three soil microbes in a metal-deficient medium. Bacillus mycoides, a heterotrophic soil microbe with no need for Mo, releases siderophores. In experiments with $B$. mycoides + glass + medium, release of Fe but not Mo is enhanced compared to abiotic experiments. Azotobacter vinelandii, a nitrogen-fixing aerobe, needs $\mathrm{Fe}$ and Mo for the nitrogenase I enzyme. When the glass is incubated with the azotobacter, enhanced release of Fe and Mo is observed. Methanobacterium thermoautotrophicum, a methanogen with a requirement for $\mathrm{Ni}$, enhances release of $\mathrm{Ni}$ from the glass. The mechanisms of metalspecific dissolution by microbes is under investigation: $\mathrm{Fe}$ in solution after siderophore-promoted dissolution of hornblende crystal is isotopically lighter than Fe released to solution without organic ligands. However, isotopic fractionation of Mo during biotic release is smaller than in the case of Fe. Investigation of such isotopic and chemical signatures related to the growth of microbes on minerals may yield new tools for the identification of life in the rock record.

\section{Polymerization of organic compounds by kinetic pathways of smectite illitization \\ Brandon Canfield $\bullet$ Arizona State University, Lynda Williams •} Arizona State University, John R. Holloway $\bullet$ Arizona State University Hydrothermal vents at the Juan de Fuca ridge are known to contain smectite-rich clay minerals. At vent fluid temperatures $\left(300-350^{\circ} \mathrm{C}\right)$ smectite quickly reacts to form illite by incorporating metal cations. It is our hypothesis that organic molecules are polymerized in the smectite interlayers during reaction to illite. Upon incorporation of metal ions and primary organic molecules in the expandable interlayer of these clays, gradual changes in the electrochemical environment may catalyze bio-oligomers that are essential components of life. Progressive steps in the catalytic process have been monitored through hydrothermal experimentation simulating seafloor and subseafloor volcanic conditions $\left(300{ }^{\circ} \mathrm{C}, 1 \mathrm{kbar}\right)$ resulting in greater than $50 \%$ illitization of the clay for reactions up to 4 weeks in length. Utilizing methanol and ethanol as the primary organic precursors, catalytic reaction products observed include alkanes, branched-phenyl compounds, carboxylic acids, and methyl-esters. More complex products appear to form as the rate of illitization increases. Organic analyses were performed by GC-MS; clay analyses by X-ray diffraction.

\section{Statistical aspects related to the detection of spatial and temporary patterns of some climate parameters around the timberline of Pico de Orizaba}

Luis Cruz-Kuri • Universidad Veracruzana, Nahum Castillo-Gonzalez • Universidad Veracruzana, José Mora-Dominguez • Universidad Veracruzana, Christopher P. McKay $\bullet$ NASA Ames Research Center, Rafael Navarro-Gonzalez $\bullet$ Instituto de Ciencias Nucleares, UNAM Pico de Orizaba $\left(19^{\circ} \mathrm{N}\right)$ has the highest treeline in the world $(4200 \mathrm{~m})$ and is the subject of an ongoing study by us supported jointly by the National Aeronautics and Space Administration (NASA), the National Autonomous University of Mexico (UNAM), and the University of Veracruz (UV). This ongoing work is related to the survival of ecosystems in extreme environments with applications to life on Mars. Related to this, we address the question of why life is constrained at high altitudes in our planet, we particularly focus on the search of those factors that determine the timberline. The treeline in temperate zones appears to be determined by summer temperatures - trees grow when the warmest summer month has an average temperature of $10^{\circ} \mathrm{C}$ or above. We hypothesize that tropical treelines, in contrast, are more sensitive to annual average temperature. In addition our current work indicates that the treeline on Pico de Orizaba reflects the temperature dependence of the nitrogen cycle. Since March 1999 we started to monitor the environmental conditions of the mountain. A detailed database is needed to quantitatively understand the current position and elevation of the treeline. The present report addresses some statistical aspects pertaining to the above undertaking, mainly from the perspective of soil temperature as well as external temperature at various locations on the mountain.

\section{Numerical models of surface moisture in the Atacama Desert}

Wanda Lorraine Davis $\bullet$ SETI Institute; NASA Ames Research Center, Christopher P. McKay $\bullet$ NASA Ames Research Center

The Atacama Desert along the Pacific Coast of Chile and Peru is one of the driest deserts in the world. During four years (1994-1998) of continuous meteorological observation in the arid core of the Atacama Desert there was only one significant rain event of $2.3 \mathrm{~mm}$ which occurred near midnight local time. Dew occurred at our station frequently following high night-time relative humidity. However, is not a significant source of moisture in the soil or under stones. Ground-water also does not contribute to surface moisture. Only the one rain event of $2.3 \mathrm{~mm}$ resulted in liquid water (required for algal growth) in the soil and beneath stones for a total of only $65-85$ hours over 4 years. Here we report on a numerical model that simulates the transport of dew and low levels of rain in undersaturated desert soil.

\section{Crystallization of the outer surface (S-layer) glycoprotein of the mesophilic bacterium Bacillus sphaericus and the hyperthermophilic Archaeon Methanothermus fervidus}

Tony Debaerdemaeker $\bullet$ Sektion Röntgen und Elektronenbeugung, Universität Ulm, Ulm, Germany, Christine Evrard $\bullet$ Université Catholique de Louvain, Louvain-la-Neuve, Belgium, Jean-Paul Declercq - Université Catholique de Louvain, Louvain-la-Neuve, Belgium, Harald Claus $\bullet$ Johannes Gutenberg-Universität, Mainz, Germany, Erol Akca $\bullet$ Johannes Gutenberg-Universität, Mainz, Germany, Helmut Koenig • Johannes Gutenberg-Universität, Mainz, Germany

For the last few years our interest has focused on the determination of the three-dimensional crystal structure of S-layer (glyco-)proteins from methanogenic Archaea of the genus Methanothermus and mesophilic, thermophilic and extremely thermophilic species of the genus Methanococcus in order to obtain a better understanding of the principles of molecular stabilization of extremely thermophilic cell surface proteins. For comparative studies, Methanococci are of special interest, since members of this genus are closely related to each other and their habitats range from mesophilic to extremely thermophilic environments. Crystals from the S-layer glycoprotein of Methanothermus fervidus were grown under microgravity conditions during the flight STS-95 of the space shuttle Discovery. We extended our research also to the domain Bacteria in order to find out whether the stabilization of these thermophilic S-layer proteins is based on the same principles as in the domain Archaea. In this case members of the genus Bacillus were chosen because the species of this genus are closely related to each other as it is the case with the Methanococci and mesophilic as well as thermophilic species exist. In the meantime, we were able to also grow crystals of the S-layer glycoprotein of Bacillus sphaericus.

\section{Analysis of bacterial community structure across varying spatial scales}

Jesse Dillon $\bullet$ University of Washington, Michael Rothrock $\bullet$ Arizona State University, Ferran Garcia-Pichel $\bullet$ Arizona State University, David Des Marais $\bullet$ NASA Ames Research Center, Brad Bebout $\bullet$ NASA Ames Research Center, David Stahl $\bullet$ University of Washington Microorganisms frequently form complex communities (microbial mats) in habitats with extreme environmental conditions (e.g. high 
temperature, salinity). We are investigating bacterial diversity in a microbial mat found in a hypersaline pond in Guerrero Negro, Baja CA, MX. This mat superficially appears to be a homogeneous community, kilometers in extent, dominated by photosynthetic algae and cyanobacteria. However, ongoing work has shown that the mat consists of a very diverse group of microbes involved in complex metabolism and biogeochemical cycling. In order to determine the scale of population variability in this system, mat core samples were taken across a range of scales ( $\mathrm{mm}$ to $\mathrm{km}$ ) along a horizontal transect. Bulk community DNA was extracted from these cores and analyzed using terminal restriction fragment analysis (T-RFLP). This technique relies upon DNA sequence differences, resulting from evolutionary divergence among groups, to assess microbial diversity. Results showed similar microbial populations across all spatial scales, however the relative abundance of community members varied across samples. Determining the spatial scale of microbial diversity is essential to our understanding of population variation in these complex microbial communities and is vital to informing our ongoing sampling efforts in understanding this environment.

\section{Biosignatures in evaporites: implications for the geological exploration of Mars}

Susanne Douglas $\bullet$ Jet Propulsion Laboratory, Heixong Yang $\bullet$ Jet Propulsion Laboratory

Death Valley offers not only some of the driest and hottest conditions on Earth but also a wide variety of different types of evaporite deposits covering a wide span of geological time. Here, we report our initial results from an investigation of the evaporite crust from the Badwater Basin and its resident endolithic microbial community. Samples were examined by environmental scanning electron microscopy (ESEM) coupled with energy dispersive X-ray spectroscopy (EDS) and X-ray powder diffraction analysis in order to learn of the inter-relationships among cells, minerals and the aqueous phase. The microbial community was seen to have a profound effect on mineral texture and on metal partitioning. The cells were enveloped in a briny layer of water, held in place by the extensive spongy mass of exopolymers they produced which was important for mineral weathering reactions. Certain elements ( $\mathrm{Si}, \mathrm{Mg}, \mathrm{K}, \mathrm{Cl}, \mathrm{Fe}, \mathrm{Mn}$ ) were present at significantly higher levels in the cells compared to the mineral; a reflection of their physiological requirements. X-ray powder diffraction and ESEM-EDS showed a microstratigraphic layering of minerals within the microbial community with a top to bottom progression of anhydrite-bassanitestrontianite-calcite. Would such biogenic geochemical alterations be measurable by remote sensing or in situ surface measurements?

\section{Hyperthermophiles of the hydrothermal vent} subsurface: limits of life and extraterrestrial analogs? Virginia Edgcomb $\bullet$ Woods Hole Oceanographic Institute, Stephen Molyneaux $\bullet$ Woods Hole Oceanographic Institute, Simone Boer $\bullet$ Woods Hole Oceanographic Institute, Karen Lloyd $\bullet$ Woods Hole Oceanographic Institute, Carl Wirsen $\bullet$ Woods Hole Oceanographic Institute, Andreas Teske $\bullet$ Woods Hole Oceanographic Institute

Hyperthermophilic archaea, isolated from deep-sea hydrothermal vents, survive and grow under extreme heat, pressure, and chemical toxicity. Defining the critical chemical and physical extremes that limit growth and survival of these organisms allows us to determine the likelihood of a deep subsurface biosphere - a possible analog for extraterrestrial habitats, and has implications for the ecology and evolution of prokaryotic life on Earth, and for searching for comparable life on other planets. We have tested growth and survival of two heterotrophic archaea under relevant in situ conditions. Both Pyrococcus sp. strain GB-D and Thermococcus fumicolans are capable of growth at 90 and $100{ }^{\circ} \mathrm{C}$ and $\mathrm{pH}$ down to 4.5 at both 1 and $250 \mathrm{~atm}$ pressure. Both could also grow at neutral $\mathrm{pH}$ in the presence of up to $30 \mathrm{mM}$ sulfide at $1 \mathrm{~atm}$, and up to $50 \mathrm{mM}$ sulfide at $250 \mathrm{~atm}$. The deep subsurface is a heterogeneous environment, and hydrothermal activity can displace organisms temporarily from their niches. Under starvation conditions both survived the above combinations of temperature and pressure plus up to $70 \mathrm{mM}$ sulfide. Sulfide reduced toxicity to free-metal ion concentrations of iron, zinc, cobalt, and copper under starvation conditions. This may indicate that sulfide serves to protect these organisms from metal toxicity in situ by complexing the free metal ions. When molarity of the free metal ion exceeds that of the sulfide, toxicity was observed.

\section{Limits of biological $\mathrm{C}$ and $\mathrm{N}$ cycling in the Atacama Desert, Chile}

Stephanie Ewing $\bullet$ University of California, Berkeley, Ronald Amundson $\bullet$ University of California, Berkeley, Kimberly WarrenRhodes $\bullet$ NASA Ames Research Center, Brad Sutter $\bullet$ NASA Ames Research Center

$\mathrm{C}$ and $\mathrm{N}$ cycling are hallmarks of life on Earth that can provide essential clues about the possibility of life on Mars. The hyperarid Atacama Desert in Chile is one of very few places on Earth where $\mathrm{C}$ and $\mathrm{N}$ cycling may be incomplete. Large deposits of nitrate salts in soils of the driest parts of the Atacama suggest that atmospherically deposited nitrate is not subject to abiotic losses by dissolution, or biological transformation and subsequent loss by processes such as denitrification. We have begun the first detailed analysis of $\mathrm{C}$ and $\mathrm{N}$ cycling in this region, measuring soil $\mathrm{C}$ and $\mathrm{N}$ pools at three sites ranging in mean annual precipitation from approximately 0 to $11 \mathrm{~mm}$. Total $\mathrm{C}$ and $\mathrm{N}$, nitrate and ammonium (inorganic $\mathrm{N}$ ), and carbonate (inorganic $\mathrm{C}$ ) are used to predict organic pool sizes prior to upcoming measurement. Total soil ${ }^{15} \mathrm{~N}$ suggests relative levels of atmospheric deposition and biological fractionation as precipitation increases. Total inorganic ${ }^{14} \mathrm{C}$ indicates the time frame of wetting events in this environment.

\section{A rapid fluorescence based viability assay for halophilic Archaea}

Thomas Fahlen $\bullet$ SETI Institute, Rocco Mancinelli $\bullet$ SETI Institute In contrast to most non-spore forming microorganisms, members of the halophilic Archaea are able to survive complete desiccation. Similar to ultraviolet (UV) radiation resistance, desiccation resistance requires either prevention or efficient repair of damage caused to nucleic acids and proteins. Interestingly, our laboratory has isolated a halophilic Archaea and shown that it is able to survive the extreme desiccation and UV radiation of the space environment. While resistance mechanisms of the radiation and desiccation resistant microorganism Deinococcus radiodurans have been characterized, thorough screens for genetic factors involved in UV and desiccation tolerance in halophilic Archaea are confounded by the relatively slow growth rate of these organisms in the laboratory. Therefore, the genetic basis (if any) of the UV and/or desiccation resistance in these microorganisms are unknown. We report here the development of a rapid, high-throughput fluorescence based assay for the effects of UV radiation on halophilic Archaea. Our results suggest that we can reduce the time required for detection of the effects of UV radiation on these organisms from weeks to minutes. This method may be applied to the rapid detection of UV sensitivity of halophilic Archaea following mutagenesis.

\section{Habitability generation by chemolithotrophic microorganisms: the Tinto River model}

David Fernandez-Remolar $\bullet$ Centro de Astrobiologia, Felipe Gomez • Centro de Astrobiologia, Ricardo Amils $\bullet$ Centro de Astrobiologia The development of ecosystems in a habitat implies the succession of more and more complex communities eventually leading to a climax community. Three aspects account for the complete development of a given ecological succession. (1) The communities modify the environment to create the habitability conditions. (2) A higher degree of diversity and stability in relation is reached. (3) Different exogenous and endogenous mechanisms of autoregulation emerge, maintaining and reinforcing the homeostasis of the ecosystem. Rarely is the homeostasis regulated endogenously, usually a complete environmental control is also present. Different studies on the Tinto River System point to a chemolithotrophic community that bio-oxidizes the Iberian Pyritic Belt, acidifying water ( $\mathrm{pH}$ between 0.9 and 3 ) and favoring high concen- 
trations of ferric iron in solution (up to $20 \mathrm{gr} \mathrm{L}^{-1}$ ) maintains this irondriven system. In spite of these extreme conditions, high microbial diversity is found in this acidic system. Acidic bacteria, archaea and eukaryotic microorganisms of the Tinto River constitute a complex community supported by algal biomass generation in highly stable hydrochemical conditions achieved by iron buffering. Moreover, iron in solution shields UV radiation. We present a model of habitability development generated by chemolithoptrophic microorganims and its astrobiological consequences.

\section{Heterotrophic microbial contamination of shocked gneiss from the Haughton impact structure, Devon Island, Nunavut, Canadian High Arctic: implications for the contamination of meteorites \\ David Fike • Scott Polar Research Institute, Charles Cockell $\bullet$ British Antarctic Survey, Pascal Lee $\bullet$ NASA Ames Research Center, David Pearce $\bullet$ British Antarctic Survey}

The Haughton impact structure on Devon Island, Nunavut is the highest-latitude terrestrial impact structure known. It provides a unique opportunity to examine the microbiological contamination of impactshocked rocks in a microbially dominated polar desert. Shocked rocks are observed to provide abundant microbial habitats, revealing that impact events can have a beneficial role in microbially dominated ecosystems. Here we describe the colonization of heavily shocked gneiss by heterotrophic bacteria in a natural setting. Porosity measurements and SEM analyses of interior pore spaces are used together with nutrient availability data to characterize the potential of the samples to support heterotrophs. The data reveal that heterotrophs are capable of penetrating to the center of heavily shocked crystalline rocks and may assist in assessing the microbiological contamination of meteorites recovered from the Antarctic ice sheet. We conclude that impact-shocked rocks could provide habitats for heterotrophic, as well as autotrophic, bacteria on the early Earth. Furthermore, because the surface of Mars is essentially an impact-shocked substrate, our data will yield insights into the ability of rocks on Mars to entrain microbes, either delivered as contamination, or more speculatively, existing as indigenous life.

\section{Whole genome sequence of the hyperthermophilic crenarchaeon Pyrobaculum aerophilum}

Sorel T. Fitz-Gibbon • University of California, Los Angeles, Malgorzata M. Slupska $\bullet$ University of California, Los Angeles, Lilit Garibyan • University of California, Los Angeles, Karl O. Stetter • University of Regensburg, Melvin I. Simon $\bullet$ California Institute of Technology, Jeffrey H. Miller $\bullet$ University of California, Los Angeles We recently published the 2.2 megabase genome sequence of Pyrobaculum aerophilum (isolate IM2), a facultatively aerobic, nitrate reducing, hyperthermophilic $\left(\mathrm{T}_{\mathrm{opt}}=100^{\circ} \mathrm{C}\right)$ crenarchaeon. This organism was chosen for sequencing on the basis of its high potential for becoming a model organism for investigation of hyperthermophiles and the Archaea.

The study of hyperthermophiles has been hampered by the lack of a robust system allowing genetic manipulations such as gene knockouts and gene additions. The $P$. aerophilum IM2 genome sequence has provided some clues which may lead to a selection system based on the organism's surprising intolerance to sulfur.

A striking feature of all completed hyperthermophilic archaeal genomes is the lack of mismatch repair gene homologs. The P. aerophilum IM2 project revealed not only a lack of mismatch repair gene homologs but also highly unstable mononucleotide runs in a pattern which is consistent with mismatch repair minus phenotypes. The mutation rate per base per replication was determined to be $2 \times 10^{-8}-10 \times 10^{-8}$, which is higher than rates reported for other organisms and similar to mutation rates in mismatch repair deficient strains. The results raise the fascinating possibility that the low mutation rate we know as "normal" in the organisms studied to date is not the only solution for life.

\section{The effects of environmental extremes on} cyanobacterial mats: UV radiation and salinity

Erich D. Fleming $\bullet$ University of Oregon, Richard W. Castenholz $\bullet$

University of Oregon

The intertidal flats near Guerrero Negro, BCS contain microbial mat communities that are dominated by a few species of cyanobacteria (i.e. Lyngbya and Calothrix). These mats spend a large portion of the year desiccated; only growing during extremely high tides or after rains. We wanted to determine how salinity and ultraviolet radiation (UVR) affect photosynthetic recovery in these mats after a prolonged period of desiccation. Dry Lyngbya and Calothrix dominated mats were excised and placed in water of varying salinity (e.g. $0,24,48$, and $80 \mathrm{ppt}$ ) under natural sunlight. Two pieces of each mat were also incubated in lagoon water (48 ppt) under UVR blocking and transmitting filters; assays were performed under UVR plus and minus conditions for both UV treatment groups. Rates of photosynthesis were determined using thin cores. Optimal photosynthetic rates were obtained in salinities of $48 \mathrm{ppt}$ for Lyngbya and $24 \mathrm{ppt}$ for Calothrix mats. However, both mat types did equally poorly in salinities of 0 and $80 \mathrm{ppt}$. There was no discernible effect of UV on the Calothrix mat. Overall, the Lyngbya mat appeared to be only affected by UVR if previously incubated under UVR minus conditions, showing a loss of UVR tolerance. When the mat was incubated under UVR plus conditions the harmful effects of UVR was minimal and photosynthetic rates were similar to those found under UVR minus assay conditions.

\section{No primary producers in the extreme arid Atacama Desert? \\ E. Imre Friedmann $\bullet N A S A$ Ames Research Center, Christopher P. McKay $\bullet$ NASA Ames Research Center, Roseli Ocampo-Friedmann • NASA Ames Research Center}

The extreme arid region of the Atacama desert (Chile) is covered by desert pavement (small stones on the surface of soil). Such pavements are common in deserts and the space under stones is a habitat for microorganisms (hypolithic habitat). Under stones which are translucent enough to transmit light sufficient for photosynthesis, cyanobacteria form a conspicuous biofilm. These microorganisms are significant primary producers in deserts, and in extreme arid areas they are the only source of organic nutrients.

We report that hypolithic cyanobacterial biofilms, ubiquitous in deserts around the world, are absent in the extreme arid region of the Atacama. Stones of the desert pavement in the Atacama are often opaque, but in some localities they are translucent, so that insufficient light can not be the cause for the absence of cyanobacteria. Nor is it the toxicity of soil, as in the laboratory algae and cyanobacteria isolated from the Negev desert grow well on extracts of Atacama soils.

Our field measurements show that there is virtually no rain, although dew is frequent. However, dew does not appear to result in moist conditions under stones. We conclude that the most probable cause for the absence of cyanobacteria is limitation of liquid water. The source of water and of organic nutrients for the sparse population of heterotrophic bacteria present in the soil is still unknown.

\section{An extreme acidic environment under the control of iron}

Felipe Gúmez • Centro de Astrobiología, Elena Gonzalez-Toril $\bullet$ Centro de Biología Molecular, Nuria Rodríguez $\bullet$ Centro de Astrobiología,

David Fernández-Remolar $\bullet$ Centro de Astrobiología, Angeles Aguilera - Centro de Astrobiología, Ricardo Amils • Centro de Astrobiología and Centro de Biología Molecular

The Tinto River (Southwestern Spain) is an extreme environment with an acidic $\mathrm{pH}$ (mean 2.3) and a high concentration of heavy metals ( $\mathrm{Fe}$, $\mathrm{As}, \mathrm{Zn}, \mathrm{Cu}, \mathrm{Ni}, \mathrm{Cr}$ ), a consequence of the active metabolism of chemolithotrophic prokaryotes thriving on the rich metallic sulfides of the Iberian Pyritic Belt. The habitat holds an unexpected level of microbial diversity, evaluated using both conventional enrichment cultures and molecular techniques (FISH, DGGE). The system is under the control of iron, a metabolic product of chemolithotrophic micro- 
organisms. The Tinto River can be considered a peculiar extreme environment generated and regulated by the biological components of the system. A preliminary geomicrobiological model of the system will be presented and its astrobiological implications discussed.

\section{Salt Plains microbial observatory and LExEn} research: characterization of halotolerant microbes William Henley $\bullet$ Oklahoma State University, Stillwater, Kelly Major • University of South Alabama, Mobile, Mark Schneegurt $\bullet$ Wichita State University, Wichita, Mark Buchheim $\bullet$ University of Tulsa, Tulsa, Robert Miller $\bullet$ Oklahoma State University, Stillwater, Andrew Potter $\bullet$ Oklahoma State University

The Salt Plains National Wildlife Refuge in Oklahoma is a minimally studied terrestrial-aquatic ecotone featuring wide fluctuations in salinity and temperature and intense solar irradiation. The salt flats are perpetually moist from Permian brine diffusely seeping to the surface, leaving behind a salt crust on the surface. We are isolating heterotrophic bacteria and archaea in media varying in salinity, $\mathrm{Mg}$ and temperature, and in media selective for diazotrophs and nitrifiers. Chlorophytes, diatoms and cyanobacteria are among the photoautotrophs isolated to date. All microbial isolates will be characterized phenetically, and RFLP and rDNA sequencing will be performed on selected unique isolates. We also will investigate the role of the recA gene in stress tolerance in bacteria. We have characterized two chlorophyte isolates with respect to salinity and thermal tolerance. At moderate temperatures, Dunaliella $s p$. tolerates saturated brine $(>30 \%$ total salts) and grows best at about $10 \%$, whereas Nannochloris $s p$. grows fastest between 0 and $5 \%$ and tolerates up to $15 \%$. SPNWR Nannochloris is more broadly halotolerant than marine congeners. At $5 \%$, Dunaliella and Nannochloris tolerate 2-h exposures to 41.5 and $45^{\circ} \mathrm{C}$, respectively. Both species exhibit greater thermotolerance at $10 \%$ compared to $2 \%$. Supported by NSF-MCB grants 9978203,0132097 , 01322083 and 0131659 .

\section{A low temperature hydrothermal system associated to the Siljan impact structure, Sweden: implications} for the search of fossil life on Mars

Tomas Hode $\bullet$ Swedish Museum of Natural History, Curt Broman • Stockholm University, Ilka von Dalwigk • Stockholm University, Sherry Cady • Portland State University, Stefan Bengtson $\bullet$ Swedish Museum of Natural History

Several lines of evidence indicate that during the early phases of planetary history (4.5-3.5 Ga), Mars was not very different from Earth. This suggests a conservative search strategy for evidence of life on Mars using the same principles that have been applied to Precambrian palaeontology on Earth.

Since thermophilic bacteria and archaea have been shown to occupy the deepest branches of the 16S rRNA-based universal phylogenetic tree of life, and hydrothermal systems were widespread on early Earth and most likely on early Mars as well, such systems are highly interesting as targets in the search for fossil (and present) life on the red planet. However, although extensive research on thermophilic organisms in hydrothermal environments has been made, reports about the fossil counterparts in general and particularly in impact-related hydrothermal systems, have occurred only sparsely (e.g. Rasmussen 2001 Nature and Westall et al. 2001 Precambrian Research).

In this study, we have investigated the impact-related low temperature hydrothermal system in Siljan, Sweden, as a possible target for the search of fossil thermophilic microorganisms. The different temperature regimes have been outlined, and suitable niches for thermophilic organisms within the system are discussed.

\section{A large-magnitude biological source of $\mathrm{H}_{2}$ to the Archaean atmosphere?}

Tori Hoehler $\bullet$ NASA Ames Research Center, Brad Bebout $\bullet$ NASA Ames Research Center, David Des Marais $\bullet$ NASA Ames Research

Center

The appearance of photosynthetic microbial communities on Earth was a critical juncture in the chemical evolution of the oceans and at- mosphere. The use of sunlight as an energy source frees life from a sole dependence on geochemical energy sources; global biospheric productivity, as well as the biological influence on planetary chemistry, can be greatly enhanced as a result. To understand the potential biogeochemical impacts of the transition to a photosynthetic biosphere, we examined chemical cycling in potential modern analogs of these Archaean photosynthetic communities-microbial mats from Baja California, Mexico. The primary geochemical role of photosynthetic members in the mat community is to extract reducing power (electrons) from water and use it to "fix" carbon dioxide into organic carbon (biomolecules). However, when exposed to a simulated Archaean atmosphere of very low oxygen content, these organisms divert a substantial fraction of the captured reducing power into the production of molecular hydrogen. Globally, this mechanism of hydrogen production could have outstripped geological sources by two to four orders of magnitude. A large-magnitude $\mathrm{H}_{2}$ flux to the environment offers the potential to enhance the global distribution and productivity of $\mathrm{H}_{2}$ consuming organisms, and also to contribute significantly to oxidation of the oceans and atmosphere via escape to space.

\section{Energy requirements of hydrogen-utilizing microbes:} boundary condition for subsurface life

Tori M. Hoehler $\bullet$ NASA Ames Research Center, Marc J. Alperin • University of North Carolina, Daniel B. Albert $\bullet$ University of North Carolina, Christopher S. Martens $\bullet$ University of North Carolina For planetary bodies with surface conditions that are too harsh to permit continuous occupation by life, the deep subsurface offers a potentially stable and habitable niche. For organisms occupying this niche, the spectrum of possible metabolisms must be limited to those which do not include sunlight as an energy source or oxygen as a chemical reagent - generally, low-energy anaerobic oxidation-reduction processes. The quantity of energy released in such processes is critical, because currently understood mechanisms of biological energy conservation indicate that energy is only "useful" to an organism when it is available at a certain minimum level - the "biological energy quantum" (BEQ). The mere existence of a BEQ implies that a significant fraction of the chemical energy present in the environment cannot be exploited by life; similarly, the absolute magnitude of the BEQ must be a key variable in determining the potential viability and distribution of subsurface microbial communities. Laboratory culture studies suggest that organisms require an energy of about $-20 \mathrm{~kJ} / \mathrm{mol}$ to grow. However, we find that hydrogen-utilizing microorganisms in an energylimited natural ecosystem are active with energy yields as low as $-10 \mathrm{~kJ} / \mathrm{mol}$. A lower BEQ would mean a significantly expanded range of energetically viable subsurface habitats for life.

\section{Temporal changes in microbial diversity and geochemistry at an active deep-sea volcano: implications for modeling the subsurface biosphere on Earth and other solar bodies}

Julie Huber $\bullet$ University of Washington, John Baross $\bullet$ University of Washington, David Butterfield $\bullet$ University of Washington

There is considerable evidence that a subsurface biosphere exists within the seafloor around hydrothermal systems, and theoretical calculations indicate that high-temperature life may also exist in the subsurface of other planets, such as Mars. With the possibility that other planets and moons may harbor hydrothermal systems, it is essential to understand the diversity of microbial life found in this unique subsurface environment on Earth. We have collected a time series of diffuse fluids from Axial Volcano to investigate the response of microbial communities to the post-eruptive evolution of the hydrothermal system. Using 16S rRNA phylogenetic analyses of particle-attached and freeliving microbial populations, we found an indigenous subsurface population of microbes related to methanogens, sulfide and methane oxidizers, as well as anaerobic heterotrophs. Hydrogen sulfide and chlorinity of vent fluids reflect a cooling of the system according to posteruptive fluid evolution models, although we continue to culture significant numbers of anaerobic hyperthermophilic archaea. Combined 
phylogenetic, geochemical, and culturing results enable development of a subsurface model to further our knowledge of how microbial communities on Earth and possibly other solar bodies are affected and constrained by the chemical and geological parameters of hydrothermal systems.

\section{Hypersaline microbial mat lipid biomarkers}

Linda L. Jahnke $\bullet$ NASA Ames Research Center, Tsegereda Embaye • SETI Institute, Kendra A. Turk • University of California, Santa Cruz, Roger E. Summons $\bullet$ Massachusetts Institute of Technology

Lipid biomarkers and compound specific isotopic abundances are powerful tools for studies of contemporary microbial ecosystems. Knowledge of the relationship of biomarkers to microbial physiology and community structure creates important links for understanding the nature of early organisms and paleoenvironments. Our recent work has focused on the hypersaline microbial mats in evaporation ponds at Guerrero Negro, Baja California Sur, Mexico. Specific biomarkers for diatoms, cyanobacteria, archaea, green non-sulfur (GNS), sulfate reducing, sulfur oxidizing and methanotrophic bacteria have been identified. Analyses of the ester-bound fatty acids indicate a highly diverse microbial community, dominated by photosynthetic organisms at the surface. The $\delta^{13} \mathrm{C}$ of cyanobacterial biomarkers such as the monomethylalkanes and hopanoids are consistent with the $\delta^{13} \mathrm{C}$ measured for bulk mat $(-10 \%)$, while a GNS biomarker, wax esters (WXE), suggests a more depleted $\delta^{13} \mathrm{C}$ for GNS biomass $(-16 \%)$. This isotopic relationship is different than that observed in mats at Octopus Spring, YSNP where GNS appear to grow photoheterotrophically. WXE abundance, while relatively low, is most pronounced in an anaerobic zone just below the cyanobacterial layer. The WXE isotope composition at GN suggests that these bacteria utilize photoautotrophy, incorporating DIC via the 3-hydroxypropionate pathway using $\mathrm{H}_{2} \mathrm{~S}$ or $\mathrm{H}_{2}$.

\section{Intrinsically and pleomorphically dwarf bacteria and archaea in a semiarid soil}

Thomas Kieft $\bullet$ New Mexico Tech, Bridget Rutz $\bullet$ New Mexico Tech Extremely small microorganisms have been of interest to astrobiologists since the report of "nanofossils" in Martian meteorite ALH84001. Dwarf microbes $(<0.3 \mu \mathrm{m})$ are common in soil; however, nearly all remain uncultivated and have not been identified. Dwarf archaea have not been studied previously. We selected a desert soil for its nutrientpoor conditions, which should favor dwarfs. Cells were eluted from soil and filtered $(0.45 \mu \mathrm{m})$. Filtrate DNA was extracted, amplified with universal bacterial and archaeal 16S rDNA primers, cloned, and sequenced. Filtrate was also inoculated into R2B medium. After incubation, cultures were filtered to select against dwarfs that grow larger in the presence of nutrients (pleomorphic dwarfs) and to select for cells that retained dwarf size (intrinsic dwarfs). A single dwarf archaea, SevArch 01, was found in the initial filtrates and in subsequent filtrate cultures, indicating an intrinsically dwarf archaea. These dwarf archaea are cocci approximately $0.25 \mu \mathrm{m}$ in diameter. Phylogenetically diverse dwarf bacteria were detected in the initial filtrates. Intrinsically dwarf bacteria in enrichment cultures were Proteobacteria that were not closely related to previously cultivated species. Pleomorphically dwarf bacteria related to Arthrobacter were detected. This study identified several novel groups of dwarf soil microorganisms.

\section{Primary structure of selected Archaeal mesophilic and extremely thermophilic outer surface layer proteins}

Helmut Koenig • Johannes Gutenberg University, Mainz, Germany, Harald Claus $\bullet$ Johannes Gutenberg University, Mainz, Germany, Erol Akca $\bullet$ Johannes Gutenberg University, Mainz, Germany, Tony Debaerdemaeker • University of Ulm, Germany, Christine Evrard • Université Catholique de Louvain, Belgium, Jean-Paul Declercq • Université Catholique de Louvain, Belgium

The archaea are recognized as a separate third domain of life together with the bacteria and eucarya. The archaea include the methanogens, extreme halophiles, thermoplasmas, sulfate reducers and sulfur metabolizing thermophiles, which thrive in different habitats such as anaerobic niches, salt lakes, and marine hydrothermals systems and continental solfataras. Many of these habitats represent extreme environments in respect to temperature, osmotic pressure and $\mathrm{pH}$ values and remind one of the conditions of the early Earth. The cell envelope structures were one of the first biochemical characteristics of archaea studied in detail. The most common archaeal cell envelope is composed of a single crystalline protein or glycoprotein surface layer (Slayer), which is associated with the outside of the cytoplasmic membrane. The S-layers are directly exposed to the extreme environment and cannot be stabilized by cellular components. Therefore, from comparative studies of mesophilic and extremely thermophilic S-layer proteins hints can be obtained about the molecular mechanisms of protein stabilization at high temperatures. First crystallization experiments of surface layer proteins under microgravity conditions were successful. Here, we report on the bio-chemical features of selected mesophilic and extremely archaeal S-layer (glyco-) proteins.

\section{Growth of methanogens on a Mars soil simulant}

Timothy Kral $\bullet$ Arkansas-Oklahoma Center for Space and Planetary Sciences, Curtis Bekkum • University of Arkansas, D. Ryan Ormond • University of Arkansas

Even though the Viking missions in 1976 found no convincing evidence of life on the surface of Mars, investigators believe that life may exist in subsurface habitats. A subsurface source of molecular hydrogen, along with carbon dioxide, and liquid water, might support the growth of methanogenic microorganisms. We have designed experiments to examine the growth of methanogens on the JSC Mars-1 simulant as a proxy for Martian soil. Washed cells of Methanosarcina barkeri, Methanothermobacter (formerly Methanobacterium) wolfeii, and Methanobacterium formicicum were added to Mars soil simulant in a carbon dioxide and hydrogen atmosphere. The total amount of water in each tube varied. We found that the soil simulant contains sufficient nutrients to support growth of all three methanogens. In order to exclude the buffer as a source of trace contaminants that might have allowed for the growth observed, methanogenic cells were washed and suspended in the liquid fraction of a Mars soil simulant/deionized water mixture. All three species grew at all concentrations tested. They also grew in concentrations of the liquid fraction by itself. In order to more fully simulate the Martian environment, future research plans include attempts to grow the methanogens at varying depths in the Mars soil simulant in a vacuum chamber at the Arkansas-Oklahoma Center for Space and Planetary Sciences.

\section{Cyanobacterial community structure in lithifying mats of a Yellowstone hot spring: implications for early Precambrian stromatolite biocomplexity}

Evan Lau $\bullet$ NASA Ames Research Center, Cody Nash $\bullet$ California Institute of Technology, Detlev Vogler $\bullet$ USDA, Ken Cullings $\bullet N A S A$ Ames Research Center

Denaturing gradient gel electrophoresis (DGGE) of partial 16S rRNA gene sequences was used to investigate the molecular biodiversity of cyanobacterial communities inhabiting various lithified morphostructures in two hotsprings of Yellowstone National Park. These morpho-structures - flat-topped columns, columnar cones, and ridged cones - resemble ancient stromatolites, which are possibly biogenic in origin. The top, middle and bottom sections of these lithified morphostructures, as well as surrounding non-lithified mats were analyzed to determine the vertical and spatial distribution of cyanobacterial communities. Results indicate that the cyanobacterial community composition of lithified morpho-structures (flat-topped columns, columnar cones, and ridged cones) were largely similar in vertical distribution as well as among the morpho-structures being studied. The phylogeny of cyanobacteria in these lithified morpho-structures were significantly different from those in surrounding non-lithified mats. These results 
provide additional support to the theory that certain cyanobacteria species, involved in the morphogenesis of lithifying morphostructures in hotsprings, may have played a role in the formation of Precambrian stromatolites.

\section{The roles of hydrodynamics and thermophilic microbes in the deposition and structuring of siliceous sinter around hot springs and geysers, Yellowstone National Park, USA \\ Donald R. Lowe • Stanford University}

Virtually all wetted surfaces within the runout systems of active, silicadepositing hot springs and geysers in YNP are populated by microbes. Below $60{ }^{\circ} \mathrm{C}$, the continuously wetted sinter surfaces are coated by thick microbial mats within which microbial filaments serve as nucleation surfaces for silica precipitation from the cooling spring waters. From $73-60{ }^{\circ} \mathrm{C}$, there is only minor localized silica deposition in spite of the wide presence of microbial mats and biofilms. In high-temperature settings, above $73^{\circ} \mathrm{C}$, there are a wide range of sinter types deposited under a variety of hydrodynamic conditions controlled by spring activity and geyser eruptive style. Although microbial biofilms widely coat sinter surfaces in these high- $T$ parts of runout systems, the main sites of silica deposition are characterized by rapid and frequent evaporation to dryness and are generally the surfaces least favorable for microbe growth. Areas that remain continuously moist and host well-developed microbial communities are generally sites of reduced or no silica deposition. Although microbes can act as surfaces for silica nucleation, both the internal microstructure and external morphology of high- $T$ siliceous sinter reflect abiotic processes related to the mechanics of surface wetting, evaporation, and drying.

\section{Studies of the culturable bacterial diversity along the north-south moisture gradient of the Atacama Desert \\ Christopher McKay $\bullet$ NASA Ames Research Center, Fred Rainey $\bullet$} Louisiana State University

The Atacama Desert, Chile is a coastal, temperate desert that stretches from $17^{\circ} \mathrm{S}$ to $28^{\circ} \mathrm{S}$ in the area between $69^{\circ} \mathrm{W}$ and $71^{\circ} \mathrm{W}$ and in the northern region is one of the driest places on Earth. From north to south there is an increase in available water and in this study we have examined the presence and diversity of culturable heterotrophic bacteria along this moisture gradient. The questions we aimed to address include : (1) Are there regions in which there are no culturable heterotrophic bacteria? (2) Are there changes in numbers and extent of diversity of culturable heterotrophic bacteria along this gradient? (3) Are there points along the gradient at which large changes in bacterial populations can be observed? Serial dilution plating methods on media for the cultivation of heterotrophic bacteria were employed. Colony forming units per gram of soil were determined and the diversity of these bacteria was examined based on $16 \mathrm{~S}$ rRNA gene sequences. The results to date indicate that (a) the numbers of culturable heterotrophic bacteria and diversity within these populations increases with an increase in water availability and (b) there are sites in the most arid region from which no or very few heterotrophic bacteria can be isolated. These sterile or near sterile soils represent environments that could be of importance in the design and implementation of planetary protection protocols.

\section{Biochemical mechanisms for surviving in acid} Mark A. Messerli $\bullet$ The Josephine Bay Paul Center for Comparative Molecular Biology and Evolution, Linda A. Amaral Zettler $\bullet$ The Josephine Bay Paul Center for Comparative Molecular Biology and Evolution, Peter J.S. Smith • BioCurrents Research Center, Marine Biological Laboratory, Mitchell L. Sogin $\bullet$ The Josephine Bay Paul Center for Comparative Molecular Biology and Evolution Contrary to former thought, organisms thrive in acidic conditions. However, most known organisms cannot tolerate acid exposure. In fact, acid is used to digest macromolecules. We are defining the biochemical nature of survival in acid by studying protists from the Rio Tinto, $\mathrm{pH}$ 1.7-2.5. Cultures of a chlamydomonad and a euglenid growing at pH 2 do not appear to have dramatically altered cytosolic chemistry as they maintain neutral cytosolic $\mathrm{pH}$ similar to related species growing at neutral $\mathrm{pH}$. This indicates that both acidophilic species maintain at least a 40000 -fold transmembrane $\left[\mathrm{H}^{+}\right]$gradient. This gradient is maintained only partly through ATP-dependent export of $\mathrm{H}^{+}$and appears to occur primarily through imposition of a barrier to $\mathrm{H}^{+}$diffusion. The cell surface $\mathrm{H}^{+}$barrier may include the plasma membrane, glycocalyx or cell wall structures. Also, the barrier may protect surface proteins by helping to maintain a neutral $\mathrm{pH}$ boundary layer at the surface of the cells. Sequence and functional analysis of the transmembrane $\mathrm{H}^{+}$ transporters will provide insight into the optimal extracellular $\mathrm{pH}$ necessary for enzyme function and therefore promote characterization of cell surface $\mathrm{pH}$. By understanding how organisms live in extremes on Earth today we can build ideas describing the development of life under the harsh conditions of early Earth and possibly extraterrestrial worlds.

\section{The isolation of novel psychrophilic microorganisms and the characterization of microbial diversity at low temperatures}

Vanya Miteva $\bullet$ Pennsylvania State University, Peter Sheridan $\bullet$ Idaho State University, Jean Brenchley $\bullet$ Pennsylvania State University Our research goal is to isolate new psychrophilic (cold-loving) microorganisms and to characterize their cold-active enzymes. As part of this work, we have enriched for bacteria from numerous cold environments. One Gram-positive Antarctic isolate of special interest grows between 0 and $21{ }^{\circ} \mathrm{C}$ and has thin rod-shaped cells that form unique balloon-like protuberances. It is phylogenetically distinct and may represent a new genus. In other work, ice core samples were inoculated into aerobic and anaerobic media and incubated at $-2{ }^{\circ} \mathrm{C}$. We used the resulting microbial communities to cultivate several morphologically distinct and often pigmented colonies. We studied the diversity of the anaerobic communities by extracting DNA, and amplifying, cloning, and sequencing over 30 different $16 \mathrm{~S}$ rRNA genes that represented at least 15 Eubacterial genera. In addition to constructing phylogenetic trees showing the relationships of these psychrophiles, this information is used to devise enrichments for represented bacteria. For example, the discovery of a $16 \mathrm{~S}$ rRNA gene sequence from a holdfast producing bacterium led to obtaining an isolate with the identical sequence. In addition to helping us understand the Earth's microbial diversity, this work develops methods and ideas for exploring extreme environments elsewhere in the universe.

\section{The long-term effects of UV exclusion on the microbial composition and photosynthetic} competence of bacteria in hot spring microbial mats Tracy B. Norris $\bullet$ Montana State University, Timothy R. McDermott • Montana State University, Richard W. Castenholz $\bullet$ University of Oregon

The primary objective of this study was to determine whether the longterm exclusion of UV radiation (UVR) from hot spring microbial mats resulted in a shift to more UV-sensitive species. Over a 1-3 month period, microbial mats in two alkaline geothermal streams in Yellowstone National Park were covered with filters that transmitted visible light and excluded or transmitted UVR. Over some, $25 \%$ transmission neutral density screens were also used. In this $40-47^{\circ} \mathrm{C}$ range, there were no apparent changes in community composition during the summer with or without high or low UVR, as assessed by DGGE profiles after PCR amplification of 16S-rRNA genes with bacteria and cyanobacteria primers. Major bands were purified from the DGGE gels and sequenced. All, but one, of the cyanobacterial sequences were unique. However, surface layers of cyanobacteria protected from UV radiation were not as competent photosynthetically as those that had been under UVR. This decrease was expressed as a loss of the ability to perform at a maximum rate under full UVR plus visible 
irradiance. However, even + UV-maintained cyanobacteria performed better when UVR was excluded during the photosynthesis tests. It is probable that the large differences in photosynthetic competence observed reflect changes at the level of gene expression in the dominant species rather than changes in species composition.

\section{Nitrogen fixation in microbial mats}

Enoma Omoregie $\bullet$ University of California, Lori Crumbliss $\bullet$ University of California, Brad Bebout $\bullet$ NASA Ames Research Center, Jon Zehr • University of California

Extant microbial mats are our best models of microbial assemblages that operated during an extremely significant period of Earth's history. Understanding the processes that influence the viability of today's extant mats allows us to gain insight into ancient microbial processes. Here we report on the preliminary findings of our investigation of nitrogen-fixation in two marine microbial mats in Guerrero Negro, Mexico. Both mats are characterized by an abundance of filamentous cyanobacteria; one by Lyngbya sp., and the other by Microcoleus sp. Our approach has been to use a combination of molecular techniques and physiological assays to identify potential diazotrophs and to characterize nifH gene expression. Using PCR and degenerate primers for nifH, we were able to recover nifH sequences from both mats, from very distinct lineages of bacteria, including gamma proteobacteria, cyanobacteria, and several less well-classified groups of anaerobic bacteria. Samples assayed for nitrogenase activity by acetylene reduction indicated that nitrogenase activity occurred in the dark. Using RT-PCR we were able to detect nifH transcripts (mRNA), which indicated that nitrogenase genes were expressed. Recovered cDNA sequences were similar to nifH sequences previously recovered from both mats, and included those from unicellular cyanobacteria.

\section{Serial analysis of rRNAs (SAR): a novel molecular strategy for sampling microbial diversity throughout the Universe}

Carmen Palacios $\bullet$ Josephine Bay Paul Center for Comparative Molecular Biology and Evolution, Dave T. Kysela $\bullet$ Yale University, Mitchell L. Sogin $\bullet$ Josephine Bay Paul Center for Comparative Molecular Biology and Evolution

The most effective means to expand our narrowed view of Earth's microbial diversity is the use of molecular techniques including the analyses of products of PCR experiments that target ribosomal RNA and other conserved universal genes. These data types provide evidence of organisms in an environment without requiring their isolation and growth in the laboratory. However, exhaustive inventories of microbial communities still remain impractical. Serial analysis of rRNAs (SAR) is a new high-throughput technique that can sample, at the sequence level, short species-specific regions of amplified rRNAs genes from many microorganisms in a single reaction. This provides a means to rapidly and efficiently enumerate relative numbers of rDNA templates in a nucleic acid population extracted from a microbial community. We will show the results of the application of this technique to an extreme environment, the hydrothermally active sediments of the Guaymas Basin (Gulf of California, Mexico). Our comparative study demonstrates the power of this technique that promises to revolutionize studies of microbial population structures. The widespread application of this technology will accelerate astrobiology studies that seek to define environmental boundaries that are compatible with the search for life on other planets, as well as the conditions conductive to life's origins and evolution.

\section{Gene expression analysis in extremophiles by DNA microarrays: identification of genes involved in the adaptation to the environment \\ Victor Parro $\bullet$ Centro de Astrobiologia, Mercedes Moreno $\bullet$ Centro de Astrobiologia, Ricardo Amils $\bullet$ Centro de Astrobiologia \\ At Centro de Astrobiología the river Tinto ${ }^{1}$ has been considered as a model ecosystem to study life based on iron. The final goal is to study}

the biological and metabolic diversity in microorganisms from Tinto, following a genomic approach, to gain insights into the mechanisms of adaptation to the environment. We selected the Gram-negative bacteria Leptospirillum ferrooxidans because it is one of the most abundant microorganisms in the river, and is one of the main ones responsible for maintenance of $\mathrm{pH}$ balance and the physico-chemical properties of the ecosystem. It manages to live in very extreme conditions such as $\mathrm{pH}$ values between 1.2 and 2.4, high metal concentrations, and nutrient requirements as minimal as ferric iron $\left(\mathrm{Fe}_{2}^{+}\right)$and $\mathrm{CO}_{2}$ as energy and carbon sources, respectively. All fragments (4.500) from an L. ferrooxidans genomic library have been printed on a chip. Gene expression analysis has been made with total RNA from $L$. ferrooxidans isolated from pure cultures under different nutritional and stress conditions. Several clones showed an altered expression pattern. After sequencing of some of them we have identified genes that might be involved in the different processes under study. Further characterization and regulation of these genes, as well as its evolutionary implications will be studied. References

${ }^{1}$ Lúpez-Archilla, A.I., Marín, I. \& Amils, R. (1993). Geomicrobiology J 11, 223.

\section{Hyperspectral data as a predictive tool for hyperthermophile community identification Jeffrey Plescia $\bullet$ United States Geological Survey}

Visible-near-infrared spectra have been used to define the spectral character of hot spring biomats in Yellowstone National Park. Each major hyperthermophile community exhibits diagnostic spectra due to the presence of Chl, Bchl and carotenoids. Data were collected at new sites to predict the hyperthermophile biota based on their spectra. Along White Creek, above Octopus Spring, Tuft Geyser) and unnamed spring were selected as test areas. Tuft Geyser erupts periodically $\left(92.8^{\circ} \mathrm{C}, \mathrm{pH}=7.7\right)$, water drains down a sinter channel and spreads across a fan (cooling to $55.6{ }^{\circ} \mathrm{C} / \mathrm{pH}=8.4$ ). The outflow channel is lined with a thin green mat that thickens and is replaced by an orange mat. Spectral data indicate that the thin green mat is a Synechococcus community without Chloroflexus; the orange mat is Phormidium. The spring pool $\left(76.7^{\circ} \mathrm{C} / \mathrm{pH}=7.2\right)$ flows into a single channel $\left(63.3^{\circ} \mathrm{C}\right)$ and then branches (cooling to $45.6^{\circ} \mathrm{C}$ ); a green mat lines the channel floor. The spectral character changes downstream with changes in morphology. The spectral pattern is identical to the Octopus Spring outflow indicating a surface Synechococcus-like community and an underlying Chloroflexus-like community. Sinter on the pool edge has numerous absorptions consistent with incorporated organics. These experiments indicate that spectral reflectance data can be used to map and define the mat biota populations.

\section{Physiological characterization of two Siberian} permafrost isolates under permafrost-like conditions Monica Ponder $\bullet$ Michigan State University, John Urbance $\bullet$ Michigan State University, James Tiedje $\bullet$ Michigan State University

Permafrost is a useful habitat for studying adaptation and long-term survival in subzero temperatures $\left(-12\right.$ to $\left.-25^{\circ} \mathrm{C}\right)$, low water activity ( $a_{\mathrm{w}}=0.9,1-7 \%$ unfrozen water), low energy availability, and longterm exposure to gamma radiation. Were have examined some of the physiological features of two common permafrost isolates, Psychrobacter (strain 273-4) and Exiguobacterium (strain 255-15), isolated from 20000-40000 and 2-3 million year old permafrost sediments respectively. The two strains grow well at $-2.5^{\circ} \mathrm{C}$ in low nutrient media with generation times of 3 and 3.5 days, respectively. The Psychrobacter exhibits extensive cell pleomorphism at cold temperatures. The pigmentation of Exiguobacteruim decreases at cold temperatures. Both the Psychrobacter and Exiguobacteruim exhibit increased freeze tolerance when grown at cold temperatures. Generation times under high salt $(1 \mathrm{M} \mathrm{NaCl})$ conditions decrease for Exiguobacteruim at $4{ }^{\circ} \mathrm{C}$ with a generation times of 10 hours compared to 14 hours without salt supplements. The genomes of both strains are being sequenced to allow a comprehensive genetic analysis of the stress adaptive traits. 
Mathematic simulation of hypersaline microbial mats: modeling effects of bacterial ecosystems on the atmosphere of early Earth

Christopher Potter $\bullet$ NASA Ames Research Center, Kelly Decker • California State University, Monterey Bay

We constructed a model to simulate $\mathrm{C}, \mathrm{O}$, and $\mathrm{S}$ cycles, and growth of cyanobacteria and sulfur bacteria in a stratified hypersaline mat. The aim was to simulate microbial effects on the atmospheric chemistry of early Earth. Inputs to the mat system include photosynthetically active radiation (PAR), near-infrared radiation (NIR), and temperature. Attenuation of PAR with each layer is modeled, and NIR attenuation depends on the abundance of bacteriochlorophyll a. The bacterial groups whose metabolism and growth are simulated include cyanobacteria (CYA), purple sulfur bacteria (PSB), colorless sulfur bacteria (CSB), and sulfur reducing bacteria (SRB). Growth of CYA occurs by either oxygenic or anoxygenic photosynthesis (AP), depending on the available substrate $\left(\mathrm{O}_{2}\right.$ or $\left.\mathrm{H}_{2} \mathrm{~S}\right)$. PSB growth occurs by AP using NIR and $\mathrm{H}_{2} \mathrm{~S}$ or by chemosynthesis $(\mathrm{CH})$, using $\mathrm{O}_{2}$ and $\mathrm{H}_{2} \mathrm{~S}$. Growth of CSB occurs via $\mathrm{CH}$, also using $\mathrm{O}_{2}$ and $\mathrm{H}_{2} \mathrm{~S}$. SRB growth occurs via anaerobic $\mathrm{CH}$ utilizing $\mathrm{SO}_{4}^{2-}$ and creating $\mathrm{H}_{2} \mathrm{~S}$ in the process. The model consists of 10 layers (1 mm each), and gains density as the biomass increases. Gas diffusion occurs between successive layers. The model was constructed in a Stellatm environment, and will be updated to include methanogens and decomposition before being programmed into an environment that is compatible with atmospheric simulations of early Earth.

Desert varnish: preservation of biofabrics/implications for Mars

Luke W. Probst $\bullet$ Bemidji State University, Bemidji, MN, Carlton C. Allen $\bullet$ NASA Johnson Space Center, Kathie L. Thomas-Keprta $\bullet$ Lockheed Martin Space Operations, Simon J. Clemett $\bullet$ Lockheed Martin Space Operations, Teresa G. Longazo • Hernandez Engineering, Mayra A. Nelman-Gonzalez $\bullet$ Wyle Life Sciences, Clarence Sams NASA • Johnson Space Center

Desert varnish is the orange to dark brown rind that accumulates on exposed rock surfaces in many arid environments. Samples from the Sonoran Desert of Arizona are composed predominantly of clays (illite, smectite) and $\mathrm{Mn}$ - and Fe-oxides (birnessite, hematite). Features that appear to be single organisms are found within the varnish and at the rock-varnish interface. Many of these features are embedded in films that strongly resemble the water-rich extracellular polysaccharides produced by diverse microorganisms. Most common are rod-shaped cell-like objects, $0.5-2$ microns in the longest dimension, located within the varnish coatings. Some of these objects are shown to contain amines by fluorescence microscopy.

The rod-shaped objects are observed in various states of degradation, as indicated by $\mathrm{C}$ and $\mathrm{S}$ abundances. Rods with higher $\mathrm{C}$ and $\mathrm{S}$ abundances appear less degraded than those with lower concentrations of these two elements. Regions rich in apparent microbes are present, while other regions display $\mathrm{Mn}$ - and Fe-rich mineral fabrics with microbe-sized voids and no obvious cells. These textures are interpreted as biofabrics, preserved by the precipitation of $\mathrm{Mn}$ and $\mathrm{Fe}$ minerals.

We are researching the preservation of biofabrics by desert varnish in Earth's geological record. Rock coatings may similarly preserve evidence of microbial life on the hyper-arid surface of Mars.

\section{Isolation of ionizing radiation resistant bacteria from arid environments}

Fred Rainey $\bullet$ Louisiana State University, John Battista $\bullet$ Louisiana State University, Christopher McKay $\bullet$ NASA Ames Research Center Alternatives to heat sterilization need to be developed for an effective planetary protection program. Sterilization using ionizing radiation and/or desiccation needs to be explored. Ionizing radiation resistance in bacterial strains such as $D$. radiodurans is an anomaly given that there are no environments on Earth with naturally high radiation levels. It has been demonstrated that prolonged dehydration causes extensive
DNA damage, and so to survive desiccation such cells require extraordinary DNA repair capability. Desiccated environments such as arid lands on Earth could be considered a natural habitat for desiccation and/or ionizing radiation resistant bacteria. The correlation between the degree of desiccation, temperature and the level of occurrence of these bacteria in natural environments has yet to be made. This would have important implications for a Mars sample return program that is considering ionizing radiation as a method of sterilization. Soil samples from the Atacama, Sonoran and Mojave Deserts have been exposed to doses of ionizing radiation between 0 and $3 \mathrm{Mrad}$. A comparison of the number of survivors and types of organisms has been made. We have shown the characteristic of ionizing radiation resistance to be more widely distributed among the bacteria than previously considered and have found desiccated soils to contain diverse populations of these organisms.

\section{Modeling halobacteria growth at very low temperatures}

Timothy Riedel $\bullet$ NASA Ames Research Center; University of Texas, Austin, Christopher McKay $\bullet$ NASA Ames Research Center, Andrew Ellington $\bullet$ University of Texas at Austin

Understanding the extreme limits of life is important in the search for life in the solar system. Identifying terrestrial organisms capable of growth in very cold, high salinity water, could be useful from a Mars perspective since liquid water may be stable on the surface of Mars if it contains enough salt to depress the freezing point and vapor pressure. Many example halobacteria can live in saturated salt solutions but most were isolated from warm environments. In order to predict the growth rates possible of extreme halobacteria at very low temperatures, we have developed thermodynamic models based on reaction kinetics. Extrapolations of these models have been compared with known psychrophile data.

\section{Spatial scale variability in cyanobacterial diversity of two contrasting microbial mat environments}

Michael Rothrock Jr • Arizona State University, Ferran Garcia-Pichel

- Arizona State University, Ulrich Nuebel • Deutsche Sammlung von

Mikroorganismen und Zellkulturen

The comparative use of three cultivation-independent methods of community structure quantification, based on $16 \mathrm{~S}$ rRNA genes, carotenoids, and morphotypes respectively, showed that when dealing with large data sets all yield similar trends. We thus confidently used $16 \mathrm{~S}$ rRNA gene-based methods to assess and compare cyanobacterial community structures from an environmentally homogeneous hypersaline pond and an environmentally heterogeneous inter-tidal area. Cyanobacterial diversity and spatial scale heterogeneity was studied by DNA extraction from the microbial mats, specific amplification of the 16S rRNA gene using cyanobacterial specific primers, and allelic separation of PCR product DNA using denaturing-gradient gel electrophoresis (DGGE). Our results show that environmental gradients influence cyanobacterial community structures. The hypersaline pond is a stable environment with no significant spatial variation in environmental parameters. Cyanobacterial diversity there remained virtually constant over all spatial scales sampled, with only minimal differences seen at the millimeter scale. While the absolute diversity was comparable to that in the hypersaline pond, spatial heterogeneity was clearly detected in the inter-tidal area along a desiccation gradient.

\section{Microbe survivability in impacts}

Eileen Ryan $\bullet$ New Mexico Highlands University, Gordon Southam • University of Western Ontario, Jennifer Lindline $\bullet$ New Mexico Highlands University, William Ryan $\bullet$ New Mexico Highlands University, Ruth Juarez $\bullet$ New Mexico Highlands University It has been suggested that comet and asteroid impacts might be a mechanism for the interplanetary exchange of microbial life. In particular, Mars meteorite ALH84001 may show possible relic biogenic activity (McKay et al. 1996). Our goal is to investigate whether bacteria 
can survive the stresses and temperatures associated with an impact event. A series of impact experiments will be conducted using Colorado Sandstone which contains a viable consortia of naturally occurring bacteria. We have characterized methods for analysis and data collection, determined the impact energies necessary for fragmentation of the rock samples, and established a protocol for the microbiological analysis.

Control samples of the sandstone have been analyzed to map the microbial distribution prior to impact, and a bacterial examination of the target relative to the impact site after the collisional event will determine if any microbes survived. Petrographic analysis will be conducted to assess the textural effects of impact and to constrain the temperature and pressure conditions within the impacted samples. Further, a numerical hydrocode (Ryan and Melosh 1998) will be used to simulate the collision and to acquire additional temperature and pressure characteristics.

References

McKay et al. (1996). Science 273, 924-930.

Ryan, E.V. \& Melosh H.J. (1998). Icarus 133, 1-24.

Investigations of microbial mineral deposition in cave environments: an analog to the Martian subsurface?

Rachel T. Schelble $\bullet$ University of New Mexico, Diana E. Northup $\bullet$

University of New Mexico, Michael N. Spilde $\bullet$ University of New

Mexico, Penelope J. Boston $\bullet$ Complex Systems Research Inc.

The subsurface may offer the only potential of discovering extant life on Mars given the hostile surface conditions. To investigate analogous Earth subsurface environments and the microbial processes active within them, we studied the microbial role in producing low-density ferromanganese deposits that coat the walls of several caves in Carlsbad Caverns National Park, NM.

The ferromanganese deposits, termed corrosion residues (CRs), may be formed in part by microbial dissolution of the limestone bedrock walls (BR). XRD analyses of CR indicates a concentration of ferromanganese deposits not present in the $\mathrm{BR}$, or the transitional layer punk rock (PR). Iron minerals such as hematite and goethite, and manganese minerals such as todorokite, and lithiophorite were identified in CR deposits. The abundance of these minerals indicates that the CR is not detrital in origin, and that bacteria may be oxidizing reduced $\mathrm{Fe}$ and $\mathrm{Mn}$ in the BR.

Investigations of the number, morphology, and metabolic activity of microorganisms present in CR, and PR were conducted using redox dyes and stains visualized with epifluorescent microscopy. The presence of metabolically active bacteria in PR samples may indicate a microbial extraction of nutrients at the PR/BR boundary. Understanding the role of microorganisms in Earth's caves may enhance our ability to search for life in the Martian subsurface.

\section{Grain growth in Taurus Molecular Cloud \\ Sachindev Shenoy $\bullet$ Rensselaer Polytechnic Institute, Douglas C. B Whittet $\bullet$ Rensselaer Polytechnic Institute, Wayne G. Roberge • Rensselaer Polytechnic Institute, George E. Hassel $\bullet$ Rensselaer Polytechnic Institute}

Observations of outflows in a molecular cloud have been associated with far infrared sources suggesting ongoing star formation and the existence of circumstellar disks. The dust grains, which get incorporated into these disks, have evolved in the molecular cloud and it is crucial to understand the properties of these dust grains, as they are the precursor to planet formation. Growth mechanisms are important as they change the size distribution and optical properties of the dust grains. It is known that coagulation and mantle formation occur in the dense environment of a molecular cloud, but no systematic study has been undertaken to see whether there exists a correlation between these two growth processes. The observational signature of the two growth processes is quite distinct and can be parameterized to understand grain growth.

To achieve this goal we focus on the Taurus Molecular Cloud (hereafter TMC) complex. We have surveyed the TMC region using the 2MASS database and have selected highly reddened field stars. We have obtained visual photometric data for these objects. Using these two datasets we have constructed the visual to infrared extinction curve along the line of sight and have used the CCM (Cardelli, Clayton \& Mathis 1989) law to evaluate the ratio of total to selective extinction. We propose to make spectroscopic observations in the $3.1 \mathrm{~mm} \mathrm{H}_{2} \mathrm{O}$ ice band to investigate grain mantle growth as a function of optical depth in the TMC. We are also investigating the IUE database to construct an extinction curve covering IR, visual and UV wavelength ranges. The UV extinction curve will help us in constraining the population of small grains along the line of sight.

This study will be a first step towards understanding the importance of the two grain growth mechanism in a molecular cloud. There are only a few stars that have extinction information and $3.1 \mathrm{~mm} \mathrm{H}_{2} \mathrm{O}$ ice data. This study will increase the dataset for which these two types of information are available and help in constraining two different growth models.

\section{Biosignatures in carbonate deposits from Mono Lake and Searles Lake, CA}

Virginia Souza-Egipsy $\bullet$ Jet Propulsion Laboratory; University of Southern California, Henry Sun $\bullet$ Jet Propulsion Laboratory;

University of Southern California, Kenneth H. Nealson $\bullet$ Jet Propulsion Laboratory; University of Southern California

Biosignatures are those components, either physical or chemical, that are consistent with life, and from which the existence of life can be inferred. They usually consist either of present or past life structural components, or chemical components (altered geology as a result of life's processes or products that life has produced). One way of understanding how biosignatures might be fixed in time is to study systems known to be of mineral and biological origin on Earth, and examine their structures and chemistry, and how these components change with time. We report here some preliminary results of the mineralization and fossilization processes in microbial sediments from shores and tufa pinnacles at Mono Lake and Searles Lake, CA. These studies have been done from both high resolution microscopy (using SEM with a backscatter detector), and microanalytical (using an EDS system) perspectives. The structures that we have seen and measured have been used to infer the origin of the carbonate particles and to understand the biological changes that occur during mineral formation. Of importance for the value of biosignatures will be two central questions: (1) is it possible for any of these structures to form abiotically; and, (2) which of the modern-day structures are conserved over time, and what are various ways in which this conservation of biosignatures might be expected to be found?

\section{Green-non-sulfur bacteria numerically dominate the photic zone of cyanobacterial mats at Guerrero Negro, Baja CS, Mexico}

John Spear $\bullet$ University of Colorado, Norman Pace $\bullet$ University of

Colorado

Microbial mats are laminated, light-driven microbial ecosystems thought to be characteristic of early Earth ecosystems. The presumed dominance of cyanobacteria in the photic zone of microbial mats, based on microscopic and culture-based studies, has never been verified with culture-independent techniques. We are using a molecular phylogenetic approach to investigate the specific composition of the photic zone of a hypersaline microbial mat in Guerrero Negro, Baja California Sur. Mat samples were obtained from the field as well as from greenhousemaintained mats housed at the NASA Ames Research Center. Community DNA was extracted, then SSU DNA genes were amplified by PCR using universal primers, cloned and partially sequenced. Of the several hundred sequences obtained so far, $30 \%$ of clones belong to the bacterial division green-non-sulfur, $25 \%$ to the cytophaga-flexibacterbacterioides division, and only $12 \%$ were identified as cyanobacteria. The remainder is composed of alpha-proteobacteria (12\%), and representatives of seven other bacterial divisions ( $3-5 \%$ each). Research is currently underway to determine whether the genomic dominance we 
detect by rDNA gene frequency is reflected in ribosomal RNA abundances.

\section{High throughput approach to study microbial phylogenetic diversity in permafrost as a terrestrial model of Mars}

Elena V. Spirina $\bullet$ Michigan State University; RAS, Pushchino, Russia, James R. Cole $\bullet$ Michigan State University, Benli Chai $\bullet$ Michigan State University, David A. Gilichinsky $\bullet$ RAS, Pushchino, Russia, James M. Tiedje $\bullet$ Michigan State University

Since Mars is cryogenic, terrestrial permafrost soil, which is inhabited by cold-adapted microbes, is a potential extraterrestrial model for investigating new approaches to studying those microbes, and to interpreting biogeochemical evidence preserved in the geological record. Two distinct environments were examined: Arctic and Antarctic permafrost. To assess microbes living in this environment, $16 \mathrm{~S}$ rDNA genes were amplified from permafrost DNA extracted both from frozen soil and those that had been incubated under both aerobic and anaerobic conditions. High throughput DNA sequencing was performed on the cloned PCR products to obtain partial 16S rDNA gene sequences. A script was written to automatically compare over 2000 partial sequences with those rrn sequences in the Ribosomal Database Project (RDP) release 8.1 using the SEQUENCE MATCH. Sequences were grouped into categories from the RDP's phylogenetic hierarchy based on the closest database match. The results suggest significant microbial diversity in all samples. However, in both regions the diversity of organisms in incubated soil was less than detected originally. Similar phylogenic groups appeared in both polar regions and some of these phylotypes have members that are known to live in other environments. The phylotypes identified here will be important organisms to study to learn their adaptations to cryogenic conditions.

\section{Extremophile genetics}

Kenneth Stedman • Portland State University, Wolfram Zillig • Max Planck Institute for Biochemistry, Christa Schleper • Technical University, Darmstadt, Erika Martusewitsch $\bullet$ Technical University, Darmstadt, John Van der Oost $\bullet$ Wageningen University

Complete genome sequence determination from many extremophiles has yielded a wealth of information, but many putative gene products have no known function and we still do not know how these organisms can thrive in their extreme environments. A very promising and powerful approach for determination of the function of unknown genes is the use of genetic tools. However, methods for gene transfer into these organisms have been lacking. The most promising candidate for the development of molecular genetics is the extremely thermophilic $\left(80^{\circ} \mathrm{C}\right)$ and acidophilic ( $\mathrm{pH} 3$ ) archaeon, Sulfolobus solfataricus. Firstly, a few mutant strains of $S$. solfataricus are available. Secondly, based on studies of the Sulfolobus virus SSV1, a viral shuttle vector was developed. Successful gene transfer using this vector was shown by bio-assay, enzymatic activity, and DNA analysis. In addition to an endogenous gene from $S$. solfataricus we were also able to functionally transfer a gene to $S$. solfataricus from the hyperthermophilic $\left(100^{\circ} \mathrm{C}\right)$ Pyrococcus furiosus. This is the first example of expression of a gene from an euryarchaeote ( $P$. furiosus) in a crenarchaeote ( $S$. solfataricus) or of any protein from any extreme thermophile in another. Further development of this molecular genetic system should allow fundamental insights into the nature of the organism and its genome.

\section{Microbial interactions in mafic rock environments Todd Stevens $\bullet$ Portland State University}

Fracture flow systems within the Columbia River Basalt group contain several contrasting habitats in which interesting biogeochemical phenomena occur. These include the proposed coupling of primary production to igneous rock weathering, which is hypothesized to allow a subsurface ecosystem independent of the surface biosphere. Laboratory microcosms are being used to study phenomena thought to be of importance in the aquifers. Methanogenic archaea, including the mesophilic Methanobacterium formicicum and the thermophilic $M$. wolfi, were able to grow and respire with $\mathrm{H}_{2}$ from anoxic basalt weathering as the sole energy source. Microorganisms appeared to enhance rock weathering, since more reduced gases were produced in inoculated microcosms than in sterile ones. Sulfidogenic bacteria were not directly able to use this energy source, since it was dependent on dissolved ferrous iron, which is removed from solution by sulfide. However, field data suggests that mixing of fluids from methanogenic fractures with sulfate-bearing fluids from ash deposits allows sulfidogens to grow by anaerobic methane oxidation. Experiments suggest that other phenomena may be spatially separated but coupled by mixing in the fracture flow system, including $\mathrm{H}_{2}$ production, iron reduction, and methanogenesis.

\section{Multi-probe life detection in Hawaiian sub-ocean deep igneous cores}

Michael Storrie $\bullet$ Jet Propulsion Laboratory, Martin Fisk $\bullet$ Oregon State University, Susanne Douglas $\bullet$ Jet Propulsion Laboratory, Gene McDonald $\bullet$ Jet Propulsion Laboratory, Radu Popa $\bullet$ Jet Propulsion Laboratory, Alexandre Tsapin $\bullet$ Jet Propulsion Laboratory

If life can exist in relatively unweathered igneous rock environments, the list of potentially habitable sites on Mars and the moons of Saturn and Jupiter expands significantly. The Hawaiian Scientific Drilling Program obtained cores to a depth of 3109 meters near Hilo, Hawaii. We report here on our multiple probe investigation of a single lithologic unit of hyaloclastite recovered from the 1335-1415 meter level. X-ray computer tomography revealed a labyrinth of interconnected vesicles. Deep ultraviolet native fluorescence images and Raman spectra obtained on fresh fractured samples without the addition of stains or fluorophores revealed signatures consistent with the activity of microbial life at vesicle rim clay-glass interfaces. These regions stained positive for DNA and chemical analysis using an electron microprobe revealed elevated levels of phosphorous. Environmental scanning electron micrographs demonstrated the presence of spherical forms 2-4 micrometers in diameter in areas exhibiting energy dispersive spectra indicative of elevated levels of carbon, phosphorous, and sulfur. We are currently performing organic analyses for potential biomarkers such as amino acids.

\section{Characterization of the process and progression of fossilization in silica depositing hydrothermal spring environments \\ Wendy Todd-Smythe $\bullet$ Portland State University, Sherry L. Cady • Portland State University}

The purpose of this research is to determine the processes involved in fossilization and the progression of fossilization in silica depositing hydrothermal spring environments. The presence of bona fide microfossils in these environments could reveal paleobiological and paleoenvironmental information about the dynamics of current and past microbial ecosystems. Bona fide microfossils include permineralized and non-mineralized cellular remains, which contain chemical and structural information indicative of cell material. Research has yielded ancient permineralized microorganisms in silica deposits displaying exceptional morphological integrity of preserved biofabrics and microbes as morphological and carbonaceous microfossils. This study will look at how one taxa of hyperthermophilic microorganism becomes fossilized in environments of hot spring ecosystems and how environmental conditions contribute to the preservation of this taxa as a bona fide microfossil. Microfossils can be seen with the use of the optical light microscope, transmission electron microscope, and the scanning electron microscope (SEM).

Biological and sinter samples collected were fixed in $2.5 \%$ gluteraldehyde. They were prepared in the laboratory for analysis with various microscopy and diffraction procedures. Results from X-ray diffraction (XRD) analyses indicate that the primary mineral present is opaline silica, SEM analyses indicate the presence of microfossils in the sinter samples. The data obtained from this research will be compiled and 
used to identify progression and processes involved in the formation of microfossils.

This research is funded by NASA's Exobiology Program, Grant \#NAG5-9579 to S. L.C.

\section{Spectroscopic characterization of the simulated Titan haze formed in a photochemical flow reactor}

Buu Tran • Rensselaer Polytechnic Institute, James Ferris • Rensselaer Polytechnic Institute, Peter Persans $\bullet$ Rensselaer Polytechnic Institute, John Chera $\bullet$ General Electric Corporate Research and Development Center

The formation of Titan haze is simulated by the ultraviolet (UV) photolysis of a mixture of gases, with mixing ratios that approximate those on Titan, in a flow system (Clarke et al. 2000). A detailed investigation of the real $(n)$ and imaginary $(k)$ parts of the complex refractive index of the polymers formed in the $0.2<\lambda<2.5$ micron region using UV-visible near-IR spectroscopy is reported for comparison with laboratory data (Khare et al. 1984) and observations from the Voyager spacecraft. The molecular structure of generated polymers has also been investigated by a combination of microscopic Fourier transform infrared (FTIR) and X-ray photoelectron Spectroscopy (XPS). UV spectral measurements suggest the presence of conjugated units of double bonds, some of which may be conjugated with other functional groups. $n$ has a value of $1.5-1.6$ throughout the $0.2-2.5$ micron region. The value of $k$ increases from 0.02 to 0.1 in the $0.2-0.8$ micron region and has values consistent with Titan haze measurements (McKay et al. 1989). FTIR established the presence of $\mathrm{CH}_{3}, \mathrm{CH}_{2}, \mathrm{C}=$ $\mathrm{C}, \mathrm{C}=\mathrm{C}^{-} \mathrm{CN}$ and possibly $\mathrm{N}-\mathrm{H}$ functional groups in the polymer. The C:N ratio in the polymer was determined to be $17.6: 1$ by XPS analysis. These laboratory findings will be valuable in understanding the data returned from the Huygens probe as it passes through Titan's atmosphere in 2004 .

\section{Incomplete nitrogen cycling in the arid core of the Atacama Desert, Chile}

Kim Warren-Rhodes $\bullet$ NASA Ames Research Center, Rocco Mancinelli - NASA Ames Research Center; SETI Institute, Ron Amundson • University of California, Berkeley, Stephanie Ewing $\bullet$ University of California, Berkeley, Brad Sutter $\bullet$ NASA Ames Research Center, Chris McKay $\bullet$ NASA Ames Research Center, Warren Belisle $\bullet$ NASA Ames Research Center

The Atacama Desert, one of the oldest and driest on Earth, provides an analog for life in dry conditions on early or present Mars. In the extreme parts of the Atacama, water scarcity inhibits microbial activity and limits the leaching of inorganic salts, thereby disrupting biogeochemical cycling. The incomplete $\mathrm{N}$ cycle in the Atacama Desert suggests that with limited to no microbial denitrification $\left(\mathrm{NO}_{3}^{-}\right.$reduction to gaseous $\mathrm{N}_{2}$ ) or leaching of nitrates, large nitrate deposits accumulated over a 10-15 Myr period. To investigate this incomplete microbial $\mathrm{N}$ cycle, acetylene blockage experiments were performed along a gradient of dry $\left(\sim 0 \mathrm{~mm} \mathrm{yr}^{-1}\right)$ to wet $\left(\sim 11 \mathrm{~mm} \mathrm{yr}^{-1}\right)$ sites, with the hypothesis that the $\mathrm{N}$ cycle closes as water availability increases. Initial results, however, indicate no statistical difference in denitrification between sites. Indeed, all results show no denitrification is occurring in the soils sampled along the climatic gradient. If the accumulation of nitrates is a general feature of extreme deserts, large deposits might be expected on the surface of Mars.

\section{Life in extreme high and low places: astrobiology in the Turpan Depression and Qinghai-Tibetan Plateau, China \\ Kimberley Warren-Rhodes $\bullet N A S A$ Ames Research Center; NRC, Chris McKay $\bullet$ NASA Ames Research Center, Lynn Rothschild $\bullet$ NASA Ames Research Center, Imre Friedmann $\bullet$ NASA Ames Research Center; NRC \\ Astrobiology searches for the limits of life on other worlds by investi- gating the extremes of our own planet. In harsh environments such as}

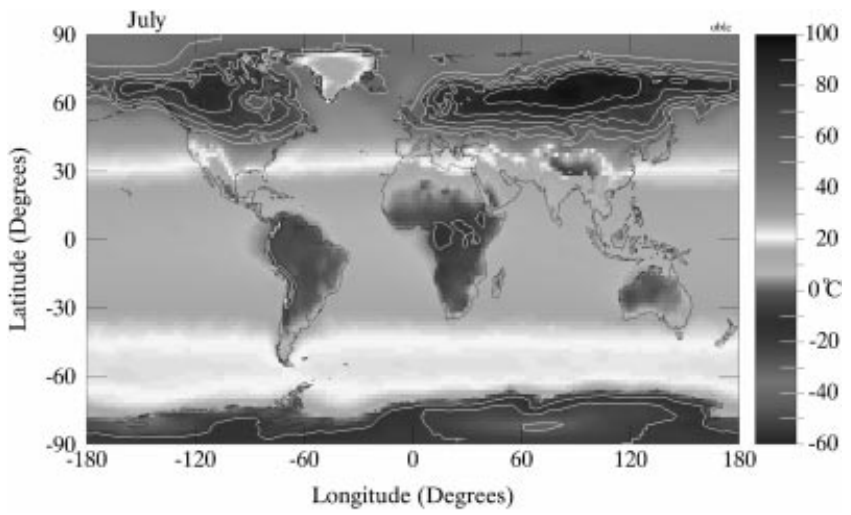

Fig. 1. Climates of Earth-like planets at high obliquity.

deserts and salt lakes, microbial communities thrive, representing possible analogs for life (extant or extinct) on Mars and Europa. Some of the Earth's most extreme environments, however, remain unexplored by the scientific community. This is particularly true for remote desert basins and high-altitude plateaus in western China. This poster presents an overview of our research on microbial communities inhabiting the hyperarid gobi (pavement) deserts and salt lakes of the Turpan Depression ( $-154 \mathrm{~m}$ ) and Qinghai-Tibetan Plateau (4000-6000 m). Novel microbial species are being isolated and identified through culturing and culture-independent methods, environmental modeling of these habitats is underway and ecological studies of the distribution of these microbial communities across varying extreme climatic, geological and UV radiation regimes are being carried out.

\section{Climates of Earth-like planets at high obliquity}

Darren M. Williams $\bullet$ Penn State Erie; The Behrend College, David

Pollard $\bullet$ Earth Systems Science Center; Pennsylvania State University The inclinations of terrestrial planet spin-axes in the Solar System are presently small, with the notable exception of Venus whose axial tilt is $178^{\circ}$. Consequently, the terrestrial seasonal cycles are mild and do not play a significant role in affecting whether these worlds are suitable for life. We now know that solar and interplanetary perturbations can cause the obliquity of Mars to increase by tens of degrees on millionyear time scales, and that Earth's spin axis would vary even more dramatically without the Moon. The hypothetical response of Earth's climate to a highly inclined spin axis has only recently undergone careful study. Here we present the results of a series of computer runs using the GENESIS 2 GCM to model Earth's climate at extreme obliquity. We find that if Earth had a Uranus-like obliquity of $90^{\circ}$, its present seasonalglobal mean temperature $\left(14.0^{\circ} \mathrm{C}\right)$ would increase slightly by $\sim 1.5^{\circ} \mathrm{C}$ owing to a very dramatic spike in temperatures over the assumed present-day northern continents shortly after the summer solstice. Fig. 1 shows that summer-time surface temperatures over Eurasia and upper North America would exceed $90{ }^{\circ} \mathrm{C}$ and over one-third of the Earth's land area would exceed $50^{\circ} \mathrm{C}$, the survivable high-temperature limit for most advanced life on Earth. This suggests that extrasolar earths with steeply inclined spin axes may have trouble supporting the quantity and diversity of life that occupies our planet today.

\section{References}

Laskar, J. \& Robutel, P. (1993). The chaotic obliquity of the planets. Nature 361, 608-614.

Laskar, J., Joutel, F. \& Robutel, P. (1993). Stabilization of the Earth's obliquity by the Moon. Nature, 361, 615-617.

Pollard, D. \& Thompson, S.L. (1997). Climate and ice-sheet mass balance at the last glacial maximum from the GENESIS version 2 global climate model. Quat. Sci. Rev. 16, 841-864.

Touma, J. \& Wisdom, J. (1993). The chaotic obliquity of Mars. Science 259, 1294-1297.

Ward, W.R. (1982). Comments on the long-term stability of the Earth's obliquity. Icarus 50, 444-448.

Williams, D.M. \& Kasting, J.F. (1997). Habitable planets with high obliquities. Icarus 129, 254-268. 
Williams, D.M. \& Pollard. D. (2000). Earth-Moon interactions: implications for terrestrial climate and life. In Origin of the Earth and Moon, eds Canup, R. \& Righter, K., pp. 513-525. Arizona Press.

\section{Constraints on microbe survivability during atmospheric transit}

David Wood $\bullet$ New Mexico Highlands University, Lacey Stewart • New Mexico Highlands University, William Ryan • New Mexico Highlands University

Naturally transporting life from one planet and seeding it on another (the "Panspermia" hypothesis) requires living organisms to survive five obstacles: (1) ejection from the parent world; (2) exposure in space; (3) atmospheric re-entry; (4) landing; and (5) the new environment. Other researchers ${ }^{1,2}$ have shown that it is possible for some bacterial species to survive the first two obstacles. Given recent discoveries of life in extreme environments, it seems likely that the fifth obstacle poses little difficulty for microbe survivability. Our objective is to assess the likelihood of microbe survivability in stages three and four. Using numerical techniques to solve a suite of differential equations for bolide deceleration, ablation, trajectory, and disruption, ${ }^{3}$ we estimate that the initial size of bolides reaching the surface at free-fall speeds are between 1 and $100 \mathrm{~m}$, depending upon composition. To further constrain the problem, we consider the pressure and temperature profiles inside the bolide as a function of distance from the leading face. We require that $>10 \%$ of the meteorite not be exposed to pressures $>100$ bar or to temperatures $>500^{\circ} \mathrm{C}$ so as not to limit microbe survivability in an intact meteorite. References

${ }^{1}$ Horneck et al. (2001). Icarus 149, 285-290.

2 Mileikowsky et al. (2000). Icarus 145, 391-427.

${ }^{3}$ Chyba et al. (1993). Nature 361, 40-44.

\section{The quest for biomolecules on Mars: molecular targets, likely habitats, and their analysis in situ by} Raman spectroscopy

David D. Wynn-Williams $\bullet$ British Antarctic Survey, Cambridge, UK†, Howell Edwards $\bullet$ University of Bradford, UK, David Dickensheets $\bullet$ Montana State University, Bozeman, MT

The search for unequivocal evidence of life on Mars is best achieved by the direct detection in situ of biomolecules in likely habitats. If microbial life ever arose during its early wet epochs, the selective pressure of solar radiation would have inevitably led to the evolution of pigments at the surface for two fundamental reasons: (1) harnessing the ubiquitous energy of photons for some sort of photosynthesis and (2) to screen or quench potentially damaging UV radiation, which disrupts many vital biomolecules in known terrestrial organisms. Pigments have characteristic molecular components, recognizable in situ in intact microbial communities by using non-disruptive laser Raman spectroscopy. Their spatial distribution is defined by unilateral light gradients and their photosynthetic and photo-protective strategies. This leads to stratification, as in lake mats and endolithic communities in rocks. Examples near the limits of life are found not only in Antarctic analogs of Martian habitats today, but also in the fossil record (e.g. hopanoids in ancient stromatolites $>2.5$ Gya in Australia). We now have Raman spectra of diverse intact communities and extracts as a basis to diagnose such biomolecules on a proposed UK-led Mars rover mission (Vanguard). This will have a miniature $1064 \mathrm{~nm}$ laser Raman spectrometer as its prime instrument, operating via side-scanning mole penetrators.

$\dagger$ David Wynn-Williams died in 2002.

\section{Adaptations of unicellular eukaryotes to extremely acidic environments \\ Linda Amaral Zettler $\bullet$ The Josephine Bay Paul Center for} Comparative Molecular Biology and Evolution, Mark Messerli $\bullet$ The Josephine Bay Paul Center for Comparative Molecular Biology and Evolution, Abby Laatsch $\bullet$ The Josephine Bay Paul Center for Comparative Molecular Biology and Evolution, Mitchell Sogin $\bullet$ The Josephine Bay Paul Center for Comparative Molecular Biology and Evolution

Astrobiology aims to understand how microbial communities thrive under extremes. The $90 \mathrm{~km}$ long Rio Tinto in southwestern Spain, $\mathrm{pH}$ 1.7-2.5, contains heavy metal concentrations several orders of magnitude above normal. The Rio Tinto's high iron concentration is thought to resemble conditions that may exist on Mars and therefore makes the river a good model for studying extraterrestrial life. Our recent smallsubunit rRNA-based studies reveal an unexpectedly high eukaryotic phylogenetic diversity in the river. These studies identify organisms that live under acid/high metal extremes but tell us little about how these organisms have adapted to such environments. The next phase of our work focuses on exploring the alterations in physiological mechanisms that might allow for growth of eukaryotic microbes at acid extremes. To this end we are isolating divergent protists such as diverse amoebae, diatoms (stramenopiles), and ciliates (alveolates). We are currently isolating and characterizing ion-transporting ATPases in a cultured chlamydomonad and euglenid and comparing these genes with those from neutrophilic counterparts. We predict that special properties of these ion transporters allow protists to survive in the Rio Tinto. Our study may provide insights into how organisms are able to adapt to harsh environments most likely resembling Earth during the first appearance of life.

\section{A molecular survey of eukaryotes from Nebraska's alkaline lakes}

Linda Amaral Zettler $\bullet$ The Josephine Bay Paul Center for Comparative Molecular Biology and Evolution, Abby Laatsch $\bullet$ The Josephine Bay Paul Center for Comparative Molecular Biology and Evolution, Brendan Keenan - University of Connecticut, Storrs, CT, Mitchell Sogin $\bullet$ The Josephine Bay Paul Center for Comparative Molecular Biology and Evolution

The lakes of the Sandhills Region of western Nebraska represent some of the most alkaline features available in the United States for investigations of life in extreme environments. By definition, environments having $\mathrm{pH}$ values greater than 10.0 are considered extremely alkaline. These alkaline lakes are diverse, ranging in salinity from fresh to brine, and are often characterized by poor drainage. While sharing similar $\mathrm{pH}$, these lakes can be distinguished by varied mineral composition (sodium, potassium, magnesium, and calcium) due to leaching and evaporation in the lakes. Most of the lakes sampled were located in the Crescent Lake National Wildlife Refuge in the Alkali Lakes region (the Sheridan and Garden Counties) of western Nebraska. We extracted genomic DNA from sediment samples collected from the above locations and created rDNA libraries. We screened these libraries for unique clones and sequenced rRNA coding regions. We summarize the eukaryotic diversity found in these lakes in a molecular phylogenetic analysis. Our data reveal a strong metazoan component to the eukaryotic community in these lakes. In addition, these environments appear to support a broad range of protists including different classes of ciliates, other alveolates, as well as stramenopiles such as diatoms, oomycetes, and chrysophytes. 


\section{Origins of life and prebiotic chemistry}

Fresh water or marine environments for the earliest forms of life? Part I: stability of primitive membranes Charles Apel $\bullet$ University of California, Santa Cruz, Pierre-Alain Monnard • University of California, Santa Cruz, David W. Deamer • University of California, Santa Cruz

Single-chain amphiphiles such as monocarboxylic acids and alcohols have been synthesized by Fischer-Tropsch-type reactions under simulated prebiotic conditions. A similar mixture of hydrocarbon derivatives is present in carbonaceous chondrites, suggesting that such compounds were likely to have been present in the early Earth environment. Previous studies have reported that single-chain amphiphiles can self-assemble into bilayer membranes in the form of vesicles. Here we explore the properties of such vesicles, in particular their stability in the presence of ionic solutes, and their ability to capture functioning macromolecules. We have found that vesicles composed of pure monocarboxylic acids are highly sensitive to $\mathrm{pH}$, but that admixtures of alcohols markedly stabilize their structure. The mixed vesicles also have the ability to capture and protect functioning macromolecules, and have a selective permeability to solutes. Salt concentrations lower than contemporary seawater disrupt these vesicles, but glycerol monodecanoate has a stabilizing effect. This suggests that if single-chain amphiphiles were major components of the first cellular membranes, then fresh water environments would be more conducive for self-assembly of bilayer structures.

\section{Emergence of selection via self-replication in peptide ecosystems}

Gonen Ashkenasy • The Scripps Research Institute, Alan Saghtelian •

The Scripps Research Institute, Reshma Jagasia $\bullet$ The Scripps

Research Institute, Yohei Yokobayashi $\bullet$ The Scripps Research

Institute, Milan Crnogorac $\bullet$ The Scripps Research Institute, M. Reza

Ghadiri $\bullet$ The Scripps Research Institute

Understanding how communities of molecular species have evolved and grown in complexity to transition from inanimate matter to living systems remains a major experimental and theoretical challenge. The existence of a self-replicating molecule was described as a prerequisite, and probably a sufficient demand, for preparing a self-reproducing system. Here we describe the design and evaluation of self-replicating peptides as the basis for self-organized molecular ecosystems. ${ }^{1} \mathrm{We}$ present our progress toward application of such a process for studying the emergence of complex "behavior" within networks that include several molecules competing for or sharing resources. ${ }^{2}$ Specifically we elaborate on the emergence of the following properties: (i) homochirality in natural compounds, ${ }^{3}$ (ii) host-parasite and reciprocal catalysis relationships in a peptide network, and (iii) selection in peptide-based arrays in which several hypercycles operate in parallel.

References

${ }^{1}$ Lee, D.H., Granja, J.R., Martinez, J.A., Severin, K. \& Ghadiri, M.R. (1996). Nature, 382, 525-528.

${ }^{2}$ Lee, D.H., Severin, K., Yokobayashi, Y. \& Ghadiri, M.R. (1997). Nature 390, 591-594.

${ }^{3}$ Saghtelian, A., Yokobayashi, Y., Soltani, K. \& Ghadiri, M.R. (2001). Nature 409, 797-801.

\section{The NSCORT/exobiology program}

Jeffrey Bada • University of California, San Diego, Gustaf Arrhenius $\bullet$ University of California, San Diego, Russell Doolittle $\bullet$ University of California, San Diego, Gerald Joyce $\bullet$ The Scripps Research Institute, Stanley Miller $\bullet$ University of California, San Diego, Leslie Orgel • The Salk Institute, Christopher Wills $\bullet$ University of California, San Diego

The NASA Specialized Center of Research and Training (NSCORT) in Exobiology is a consortium of scientists at three institutions in the La Jolla area of San Diego, CA. These groups have conducted a diverse set of investigations, which we will only highlight here. Dr Jeffrey Bada $e t$ al. are studying sea water, polar ice, lunar soils, carbonaceous chondrites, and meteorites from Mars to determine the importance of extraterrestrial organic material on the primitive Earth. Dr Gustaf Arrhenius et al. are studying the oldest chemofossils so far identified, consisting of graphitized organic matter in early Archean $(3.8 \mathrm{Ga})$ metamorphosed sedimentary rocks in southern West Greenland Isua formation. Dr Russell Doolittle et al. are investigating the tangled roots of the "shrub" of life by means of amino acid sequence comparisons. Dr Stanley Miller et al. are investigating the compounds formed from nucleotide bases reacting with the products of prebiotic processes. Dr Leslie Orge et al. are studying self-replicating polymers simpler than RNA, in particular the catalysis of peptide replication by mineral surfaces. Dr Gerald Joyce et al. are performing experiments on the evolution of instructed protein synthesis in the context of a genetic system based on RNA genomes and RNA catalysts. Dr Christopher Wills et al. are examining the early evolution of DNA microsatellites.

\section{Chiral amino acid synthesis in simulated interstellar} ices

Max Bernstein $\bullet$ The SETI Institute, Jason Dworkin $\bullet$ The SETI

Institute, Scott Sandford $\bullet$ NASA Ames Research Center, George Cooper $\bullet$ NASA Ames Research Center, Louis Allamandola $\bullet$ NASA Ames Research Center

In the interest of better understanding the formation and distribution of organic molecules in space and the impact they may have had on the origin and evolution of life on Earth (and perhaps planets of other star systems), we perform ice processing experiments in the laboratory. We will report on how amino acids and other interesting organic molecules, of the kinds seen in meteorites and interplanetary dust particles (IDPs), can be made under conditions common in dense molecular clouds in the interstellar medium.

We have previously reported our syntheses of various functionalized polycyclic aromatic hydrocarbons ${ }^{1,2}$ including naphthoquinone ${ }^{3}$ and of vesicle-forming amphiphiles. ${ }^{4}$ We now report ${ }^{5}$ the amino acids glycine, alanine, and serine were formed in the laboratory by the UV photolysis of realistic interstellar ice analogs, composed primarily of $\mathrm{H}_{2} \mathrm{O}$, and including $\mathrm{CH}_{3} \mathrm{OH}, \mathrm{NH}_{3}$, and $\mathrm{HCN}$. N-formyl glycine, cycloserine and glycerol were detected before hydrolysis, and glycine, racemic alanine, racemic serine, glycerol, ethanolamine, and glyceric acid were found after hydrolysis. This suggests that some meteoritic amino acids (and other molecules) may be the direct result of ice photochemistry, challenging the paradigm that they formed by reactions in liquid water on meteorite parent bodies. Ice photochemistry may more easily accommodate the deuterium enrichment, and enantiomeric excesses of the amino acids in meteorites.

You can learn more about the astrochemistry lab at http:// www.astrochem.org.

References

${ }^{1}$ Bernstein, M.P., Sandford, S.A., Allamandola, L.J., Gillette, J.S., Clemett, S.J. \& Zare, R.N. (1999). UV Irradiation of polycyclic aromatic hydrocarbons in ices: production of alcohols, quinones, and ethers. Science 283, 1135-1138.

${ }^{2}$ Bernstein, M.P., Elsila, J.E., Dworkin, J.P., Sandford, S.A., Allamandola, L.J. \& Zare, R.N. (2002). Addition of side groups to the PAH coronene by UV photolysis of cosmic ices in the laboratory. In preparation.

${ }^{3}$ Bernstein, M.P., Dworkin, J.P., Sandford, S.A. \& Allamandola, L.J. (2001). Ultraviolet irradiation of naphthalene in $\mathrm{H}_{2} \mathrm{O}$ ice: implications for meteorites and biogenesis. meteoritics and planetary Science 36, 351-358.

${ }^{4}$ Dworkin, J.P., Deamer, D.W., Sandford, S.A. \& Allamandola, L.J. (2001). Self-assembling amphiphilic molecules: synthesis in simulated interstellar/precometary ices. Proc. Nat. Acad. Sci. 98, 815-819.

${ }^{5}$ Bernstein, M.P., Dworkin, J.P., Sandford, S.A., Cooper, G.W. \& Allamandola, L.J. (2002) The formation of racemic amino acids by ultraviolet photolysis of interstellar ice analogs. Nature in press. 


\section{Mineral-mediated abiotic nitrogen reduction under hydrothermal conditions}

Jay Brandes $\bullet$ University of Texas, Austin, Robert Hazen $\bullet$ Carnegie Institution, Hatten Yoder, Jr. • Carnegie Institution

Prebiotic sources of ammonia may have facilitated synthesis of amino acids and other essential biomolecules. We studied catalytic abilities of natural sulfide and oxide minerals in reducing aqueous nitrate and nitrite solutions. We seal $15 \mathrm{mg}$ of $10-\mathrm{mM}$ solutions $\left(\mathrm{NaNO}_{2}, \mathrm{NaNO}_{3}\right.$, or $\mathrm{NH}_{4} \mathrm{Cl}$ ( $\pm 5 \mathrm{mg}$ powdered mineral) into gold tubes and subject them to $300^{\circ} \mathrm{C}$ and $50 \mathrm{MPa}(\sim 500 \mathrm{~atm})$ for $90 \mathrm{~min}$ to $24 \mathrm{~h}$ in a gas-media, high-P apparatus.

Transition metal sulfides [FeS, $\mathrm{FeS}_{2}, \mathrm{NiS}, \mathrm{Cu}_{2} \mathrm{~S}, \mathrm{CuS}, \mathrm{CuFeS}_{2}$, $\left.\mathrm{CuFe}_{2} \mathrm{~S}_{3}, \mathrm{Cu}_{5} \mathrm{FeS}_{4}, \mathrm{PbS}, \mathrm{ZnS},(\mathrm{Ni}, \mathrm{Fe})_{8} \mathrm{~S}_{9}, \mathrm{Cu}^{* *} 2 \mathrm{FeSnS}_{4}, \mathrm{WS}_{2}\right]$ rapidly convert nitrogen oxides to ammonia, with up to $90 \%$ conversion in $90 \mathrm{~min}$. The conversion efficiency is lower in general for nitrite, which is less stable than nitrate under these conditions. The greatest conversion rate is observed in pyrrhotite, as expected from our earlier work [Brandes et al. (1998) Nature 395, 365]. Oxide minerals $\left(\mathrm{Fe}_{3} \mathrm{O}_{4}, \mathrm{NiO}, \mathrm{Cu}_{2} \mathrm{O}, \mathrm{MnO}\right)$ may also reduce nitrate and nitrite to ammonia, albeit with lesser yields than corresponding sulfides.

Ammonium in aqueous solution is stable at $300{ }^{\circ} \mathrm{C}$ and $50 \mathrm{MPa}$ in the presence of these sulfide and oxide minerals. We observe no significant loss in $24 \mathrm{~h}$. We conclude that ammonia is not rapidly destroyed under hydrothermal conditions and that deep-ocean hydrothermal systems provided a significant source of ammonia for the Archaean Earth.

\section{Anharmonic vibrational spectroscopy of small} biological building blocks: amino acids, dipeptides, and their complexes with water

\section{Galina Chaban $\bullet$ NASA Ames Research Center, Benny Gerber •}

University of California, Irvine

Vibrational spectroscopy is one of the most powerful tools that can help identification of molecules in space. Identifying small biological molecules in the interstellar medium is very important from the point of view of the origin of life, since all the material on Earth originated from primordial interstellar particles. To assist in interpretation of the observed infrared spectra, it is crucial to provide accurate theoretical data. In this work, we use a vibrational self-consistent field technique, combined with direct use of accurate ab initio potentials, to calculate anharmonic vibrational frequencies and infrared intensities for the simplest amino acid glycine and its complexes with water. Comparison with available experimental data shows very good agreement between the calculated and experimental results. Effects of complexation with water on vibrational spectra are considered and found to be very significant. In order to obtain accurate anharmonic vibrational spectra for larger amino acids and dipeptides, we employ hybrid (QM/MM) potentials, where key parts of molecular systems are treated at an $a b$ initio level, while side chains are represented with empirical force fields. The calculated vibrational spectra of glycine, alanine, and valine and their complexes with water can be directly compared with observational experimental data, and assist in their identification in space.

\section{Analysis of amines in meteorites using fluorescence molecular probes}

Simon Clemett $\bullet$ Lockheed Martin, Scott Messenger $\bullet$ Washington University, Kathie Thomas-Keprta $\bullet$ Lockheed Martin, Susan Wentworth $\bullet$ Lockheed Martin, Georgann Robinson $\bullet$ Bay Tech, David McKay $\bullet$ NASA Johnson Space Center

We report the first measurements on the spatially resolved molecular distribution of primary amines in a meteoritic sample. The technique is fast, non-invasive, and mineralogically nondestructive and can be used in conjunction with elemental and isotopic measurements. The molecular analysis of meteorites using specific fluorescent molecular probes is feasible and preliminary experiments suggest that it has the potential to help resolve whether the carrier phase(s) for isotopic anomalies is (are) organic in nature.

Our approach is to target specific organic monofunctional groups and tag them with a fluorescent molecular probe. Once a sample surface has been tagged the spatial distribution of those monofunctional groups can be determined by fluorescence microscopy. This same surface can then subsequently be analyzed by two-step laser microprobe mass spectrometry to determine the specific molecular species tagged.

Murchison (CM2) was chosen as a model substrate. The distribution of primary amines was then determined using an Olympus BX60 fluorescent microscope equipped with an image intensifier and CCD array. The amine distribution is correlated with $\mathrm{Si}$ and $\mathrm{O}$ but anticorrelated with $\mathrm{Fe}$ and $\mathrm{S}$. The usefulness of the technique should be applicable to a wide range of samples in the fields of cosmochemistry and astrobiology.

\section{Enantiomeric and isotopic analysis of sugar derivatives in carbonaceous meteorites}

George Cooper $\bullet$ NASA Ames Research Center, Cynthia Asiyo • NASA Ames Research Center, Kendra Turk $\bullet$ NASA Ames Research Center

Among the classes of organic compounds found in carbonaceous meteorites are amino acids, carboxylic acids, hydroxy acids, purines, and pyrimidines. Such compounds are thought to have been delivered to the early Earth in asteroids and comets and may have played a role in the origin of life. ${ }^{1}$ Likewise, sugar derivatives are critical to all known lifeforms. Recent analyses of the Murchison and Murray carbonaceous meteorites revealed a diverse suite of such derivatives, i.e. sugar alcohols, and sugar acids. ${ }^{2}$

Our current project is the analysis of the enantiomeric $(\mathrm{D}, \mathrm{L})$ and isotopic $(\mathrm{C}, \mathrm{D}, \mathrm{O})$ composition of individual sugar derivatives found in carbonaceous meteorites. $\mathrm{C}$ and $\mathrm{D}$ isotope analysis of meteoritic sugar alcohols (glycerol, threitol, ribitol, etc.) as a group revealed that they were indigenous to the meteorite and not the result of contamination. ${ }^{2}$ Preliminary C-13 analysis of an individual compound, glyceric acid, show that it is also extraterrestrial. Full details of this and other analyses will be presented.

\section{References}

1 Cronin, J.R. \& Chang, S. (1993). In Chemistry of life's Origins, eds Greenberg, J.M., Pirronello, V. \& Mendoza-Gomez, C. pp. 209-258. Kluwer, Netherlands.

2 Cooper, G., Kimmich, N., Belisle, W., Sarinana, J., Katrina Brabham, K. \& Garrel, L. (2001). Sugar-related organic compounds in carbonaceous meteorites. Nature 414, 879-883.

\section{Organic solids color the icy bodies of the outer solar system}

Dale Cruikshank • NASA Ames Research Center

Ice-rich bodies in the outer Solar System include the large satellites of the giant planets, Jupiter through Neptune, the rings of Saturn, the comets, Pluto-Charon, and the objects that populate the Kuiper disk and the Oort cloud. Many of these objects have surface exposures of $\mathrm{H}_{2} \mathrm{O}$, while Neptune's satellite Triton has surface exposures of at least five different ices $\left(\mathrm{N}_{2}, \mathrm{CH}_{4}, \mathrm{CO}, \mathrm{CO}_{2}\right.$, and $\left.\mathrm{H}_{2} \mathrm{O}\right)$ that have been identified by remote sensing observations. The organic materials produced by ice processing, and other complex organics of exogenous origin are of special interest in the context of materials delivered to the prebiotic Earth and other planets. This paper reports on new astronomical and spacecraft observations of icy bodies, and the modeling efforts to determine the nature of the organic materials that affect their spectral reflectance in the ultraviolet, visible, and near-infrared spectral regions. This work is tied to laboratory investigations of natural (from meteorites) and synthesized organic solids, and the measurements of their complex optical refractive indices used in the spectral modeling.

\section{Calculation of the thermodynamic properties of intermediates in a prebiotic reverse citric acid cycle and prediction of their equilibrium activities in high P-T sea-floor hydrothermal fluids}

Peter Dalla-Betta $\bullet$ Arizona State University, John Holloway • Arizona State University, Peggy O'Day • Arizona State University

Among the most essential and conserved of metabolic pathways is the 
citric acid cycle (CAC). The CAC is thought primarily to have operated in reverse to supply precursors for amino acid, sugar, lipid, and heme synthesis. Sea-floor hydrothermal (SFH) systems have been hypothesized as a setting where conditions are feasible to drive a reverse CAC. However, since SFH are defined by high $P-T$ conditions, the accurate prediction of reaction thermodynamics in these systems requires knowledge of the each compound's thermochemical properties at $P$ and $T$. Testing the convention that the activity of n-alkylthiols in (SFH) vent fluid act as carbonyl activators, and that high concentrations of $\mathrm{H}_{2}$ acts to drive the production of pyruvate from acetic acid and $\mathrm{CO}_{2}$ to begin the cycle, individual reactions will be assessed to determine their feasibility and establish thermodynamic barriers. The infinite dilution thermochemical properties of compounds are used if data is available and predicted with group contribution methods when none exists. Gibb's free energy of hydration is used to predict the volumetric and heat capacity functions for the revised HKF equation of state at elevated $P$ and $T$. EQ3/6 is then used to predict the equilibrium composition and activity of intermediates over a range of $\mathrm{SFH}$ temperatures constrained by the FMQ mineral assemblage.

\section{Effect of surface chemistry on mineral-promoted abiotic organic synthesis in early terrestrial planet hydrothermal systems}

Tamara Diedrich • Arizona State University, John Holloway $\bullet$ Arizona State University, Thomas Sharp • Arizona State University

Seafloor hydrothermal systems are one promising environment for the emergence of life on Earth and may serve as analogs to environments of early life on other terrestrial planets. Previous experimental results suggest the potential for abiotic organic synthesis under seafloor hydrothermal conditions. Voglesonger et al. ${ }^{1}$ found methanol production from a gas-rich $\mathrm{CO}_{2}-\mathrm{H}_{2}-\mathrm{H}_{2} \mathrm{O}$ mixture to be strongly influenced by the mineral substrate used, with significant conversion to $\mathrm{CH}_{3} \mathrm{OH}$ occurring over magnetite + quartz.

We have initiated research to further examine the effect of specific mineral surfaces on abiotic organic synthesis under seafloor hydrothermal conditions. The first mineral being studied is magnetite. We have synthesized magnetite in three distinct morphologies by modifying a solvothermal method described by Dubois et al. ${ }^{2}$ The variation in morphologies: octahedral, skeletal, and dodecahedral, was produced using different magnetite precursors. We hypothesize that these morphologies, each displaying a unique set of surfaces, will react differently in promoting abiotic organic synthesis in experiments under seafloor hydrothermal conditions.

References

${ }^{1}$ Voglesonger, K.M., Holloway, J.R., Dunn, E.E., Dalla-Betta, P.J. \& O’Day, P.A. (2001). Chemical Geology 180, 129-139.

2 Dubois, T. \& Demazeau, G. (1994). Materials Letters 19, 38-47.

\section{A proteomic and genomic investigation of the Archaeon methanosarcina thermophila}

Yan-Huai R. Ding • Pennsylvania State University, James G. Ferry • Pennsylvania State University, Shiping Zhang • E. I. DuPont de Nemours Company, Jean-Francois Tomb $\bullet$ E. I. DuPont de Nemours Company

M. thermophila is a methane-producing archaeon that contains enzymes thought to have evolved during the origin of life. Expression profiles of the total soluble proteins from acetate-, methanol- and trimethylamine (TMA)-grown cells in M. thermophila were investigated with twodimensional SDS-PAGE (2-D) and N-terminal analysis. Three homologs of the methanol:coenzyme M methyltrans-ferase 1 (MT1) and nine homologs of the methylcobalamin :coenzyme M methyltransferase 2 (MT2) genes were identified in the genome of M. thermophila. All three homologs of MT1 were expressed abundantly in methanolgrown cells, and one homolog of MT1 was expressed in acetate-grown cells. One of nine homologs of the MT2 gene product was found in methanol-grown cells. Analysis of the deduced amino acid sequence of the MT1 homologs showed that they were highly conserved. Additionally, a bacterial-type heat shock protein (GroEL) was identified in
TMA-grown cells. This is the first report of a bacterial-type GroEL gene expressed in vivo in the Archaea domain. Sequence analysis showed that the M. thermophila gene encoding GroEL is highly conserved with the gene from Methanosarcina acetivorans, Methanosarcina barkeri and organisms from the Bacteria domain. Phylogenetic analysis indicated that the Methanosarcina GroEL gene could have been acquired by horizontal gene transfer from the Bacteria domain.

\section{A mechanism for the co-production of protobacterial and protoviruses by aerosol fission \\ Jamie Donaldson $\bullet$ University of Toronto, Adrian Tuck $\bullet$ NOAA \\ Aeronomy Lab, Veronica Vaida • University of Colorado \\ We examine the prebiotic applicability of our recent analysis of the fission of an atmospheric aerosol particle coated with an organic film. The fission is made possible by the free energetics of compression of the exterior monolayer film on the parent particle, which overcomes the increase in surface area associated with the production of two spherical daughter particles. Asymmetric division into a larger and a smaller particle is expected to follow surfactant film collapse. The size of the parent particle is determined by the balance between aerodynamics and gravity, while the ratio of the radii of the daughters is determined by the compression characteristics of the amphiphilic molecules comprising the parent film. For an Earth atmosphere of 1 bar surface pressure, the larger and smaller daughters have the sizes of a single-celled bacterium and of a virus respectively. Chemical differentiation between the daughters is possible.}

\section{Clay-catalyzed synthesis of RNA: a laboratory model of the origins of the RNA world}

James Ferris $\bullet$ Renssealer Polytechnic Institute, Troy, $N Y$

The clay mineral-catalyzed formation of 6-14 mers of RNA has been described. In this study we report the elongation of a 10 mer primer to $30-50$ mers. Elongation of the primer ${ }_{32} \mathrm{pdA}(\mathrm{pdA}){ }_{8} \mathrm{pA}$ proceeds by the reaction of the $5^{\prime}$-phosphorimidazolides of adenosine and uridine in the presence of montmorillonite clay. Daily addition of the activated nucleotides for up to 14 days results in the formation of 40-50 mers using the 5'-phosphorimidazolide of adenosine (ImpA) and 25-30 mers using the 5 -phosphorimidazolide of uridine (ImpU). Alkaline phosphatase hydrolysis studies revealed that some of the oligomers are capped at the $5^{\prime}$-end to form, with ImpA, $\mathrm{Ap}_{32} \mathrm{pdA}(\mathrm{pdA})_{8} \mathrm{pA}(\mathrm{pA})_{\mathrm{n}}$. The extent of capping depends on the reaction time and activated mononucleotide used. Hydrolysis with ribonuclease $\mathrm{T}_{2}$ followed by alkaline phosphatase determined the sites of the $3^{\prime}, 5^{\prime}$ - and 2', 5'-phosphodiester bonding to the primer. Recent studies indicate that use of different activated monomers result in the formation of long RNAs in 3-6 days. The potential significance of the mineral catalyzed formation of long oligomers of RNA to the origin of life based on RNA (the RNA world scenario) is discussed. Supported by NASA grants NAG5-7598, NAG59607 and NSF grant CHE-0076281.

\section{The New York center for studies on the origins of life: a NASA NSCORT}

James Ferris $\bullet$ Rensselaer Polytechnic Institute

This center is conducting an interdisciplinary program on the origins of life. There are seven participants from RPI, SUNY Albany and The College of St Rose working in the areas of astrophysics, planetary geology, chemistry and molecular biology. There are interdisciplinary overlaps between this continuum of fields where this research is being carried out as well as in the disciplines. Some students have more than one advisor since they are conducting interdisciplinary research. Some students are getting degrees in interdisciplinary science. There is a vibrant student-post doc Journal Club, which discusses research and meets with the seminar speakers after the weekly seminar. The outreach program includes interviews with the seminar speakers that are broadcast on National Public Radio. In addition, the audio portion of the seminar presentations together with the visuals used by the speaker can be downloaded from our website (www.origins.rpi.edu). The recent 
seminar speakers and the titles of their lectures are listed on the poster. Students, postdoctorals and faculty participants from the Center will be present at this meeting if you have questions. Supported by NASA grant NAG5-4546.

\section{Assembly of astrochemicals \\ Johannes Fraaije $\bullet$ Leiden University, Alexander Kros $\bullet$ Leiden \\ University, Pascale Ehrenfreund $\bullet$ Leiden University}

This report discusses results from the combination of two research programs essential to the new discipline of astrobiology: simulation of prebiotic chemical reactions and the definition of organic matter in space. In the first approach, we present the spontaneous nanogel formation of non-biological amphiphilic polymers. The amphiphiles not only constitute cosmetics, but are also present in certain meteoritic samples, which makes them interesting from different points of view! The nanogels offer a fascinating glimpse for a novel approach in prebiotic chemistry, in which confinement of chemicals is controlled by microphase behaviour of simple block copolymers. In the second approach, analysis of ISO data provides evidence for new species on interstellar dust particles and how their composition is changing according to the environmental conditions. Observational results from ground- and space-based observatories in combination with laboratory simulations allow us to monitor the evolution and transformation of cosmic dust from molecular clouds until their incorporation into Solar System material. The comparison of abundances and isotopic ratios of dust and molecules in different space environments provides important constraints for the chemistry in the outer Solar System and the extraterrestrial delivery of organic species to the early planets.

\section{Pragmatic emergence: complexity theory as a guide to origin-of-life experiments \\ Robert Hazen $\bullet$ Carnegie Institution}

Emergent phenomena include systems such as sand dunes, ant colonies and consciousness that arise from the interaction of agents (e.g. molecules, cells, organisms). The seductive search for universal organizing principles of complex systems drives many researchers, but major hurdles must be overcome. In particular, no universally applicable quantitative definition of complexity has been devised. Nevertheless, the analogous behaviors of many emergent systems point to common features that inform origin-of-life experiments.

A pragmatic application of complexity theory to studies of life's origin and distribution leads to several conclusions.

1. Life began as a series of emergent steps; each added molecular complexity and organization.

2. Steps in the transition from non-life to life include synthesis of biomolecules; molecular selection/organization; emergence of self-replicating cycles; molecular natural selection.

3. Origin-of-life studies can be simplified by treating each step as a separate experimental program.

4. The search for biomarkers should include "abiomarkers"emergent chemical and isotopic signatures that otherwise would have been erased by life.

5. Complexity is enhanced by energetic interfaces such as mineral surfaces.

6. Complexity is enhanced by cycling energy gradients. Thermal, evaporation and other cycles in the origin of life deserve additional study.

\section{Simulation of shock chemistry in comet and meteor impact}

Winifred M. Huo $\bullet$ NASA Ames Research Center, Christopher E. Dateo $\bullet$ Eloret Corporation; NASA Ames Research Center, Christopher P. McKay $\bullet$ NASA Ames Research Center, William J. Borucki $\bullet N A S A$ Ames Research Center

In their study of organic synthesis from impact shocks using the laserinduced-plasma (LIP) technique, McKay and Borucki ${ }^{1}$ found that organic synthesis preferentially occurred in a reducing gas mixture rich in methane, and not in a mixture rich in carbon dioxide. This result means chemical models based on the thermodynamical equilibrium approach do not apply to shock chemistry. In this study, we employ the technique of reacting flow, i.e. chemical kinetics in a fluid flow, to simulate the chemistry occurring in LIP and in the wake region from a comet or meteor impact. Three different air compositions have been used: (1) $1 /{ }_{3} \mathrm{CO}_{2}$ and ${ }^{2} /{ }_{3} \mathrm{H}_{2}$, (2) pure $\mathrm{CH}_{4}$, and (3) ${ }^{1} /{ }_{4} \mathrm{CH}_{4},{ }_{1}^{1}{ }_{4} \mathrm{CO}_{2}$, and $1 /{ }_{2} \mathrm{H}_{2} \mathrm{O}$. The stoichiometric ratio of gas mixtures (1) and (3) are kept the same. For (1) we obtain equal mole fractions of $\mathrm{CO}$ and $\mathrm{H}_{2} \mathrm{O}$ as the major products and for (2) $\mathrm{C}_{2} \mathrm{H}_{2}$ is the major product. In both cases our results are in agreement with Ref. (1). For (3) we find an interesting case where the nature of the chemicals produced to be critically dependent on the flowfield temperature. At the higher temperature part of the wake region, $\mathrm{CO}$ and $\mathrm{H}_{2} \mathrm{O}$ are the dominant products, whereas in the cooler region $\mathrm{C}_{2} \mathrm{H}_{2}$ is the dominant product. Further studies of these reactions, as well as for the gas mixture including $\mathrm{N}_{2}$, are being pursued. References

${ }^{1}$ McKay, C.P. \& Borucki, W.J. (1997). Science 276, 390-392.

\section{The meteoric rise from extraterrestrial organics to prebiotic molecules on the early Earth}

Peter Jenniskens $\bullet$ SETI Institute, Ray Russell $\bullet$ The Aerospace Corporation, George Rossano $\bullet$ The Aerospace Corporation, Christophe Laux $\bullet$ Stanford University, Iain Boyd $\bullet$ University of Michigan, Rick Rairden $\bullet$ Lockheed Martin, Olga Popova $\bullet$ RAS

We will briefly summarize the various pathways that are currently being considered along which organic compounds in solar system bodies may have been delivered to an organic-poor early Earth. We will briefly mention the merit of protoplanetary accretion, comet and asteroid impacts in a late veneer, the infall of meteorites and dust, and the accretion of extraterrestrial matter in the form of meteors. We will briefly describe how recent campaigns to study the Leonid meteor storms have changed our understanding of the physical conditions in meteoric ablation and the chances for organic matter to survive this process. First results from the 2001 Leonid Multi-Instrument Aircraft Campaign are discussed. We will present the latest measurements of plasma temperatures and heating timescales and new rarefied flow models to explain the observations. The models show the potential pathways along how organic molecules can survive the ablation process, albeit chemically altered. Efforts to find the waste products of organic matter decomposition have led to upper limits of the abundance of $\mathrm{H}$, $\mathrm{CN}$ and $\mathrm{C}_{2}$. Finally, we will discuss the evidence for the survival of solid debris in even Mach 270 flows, debris that may still contain organic material. We will also discuss how efficient meteors may be in changing the atmospheric composition of the early Earth's atmosphere.

\section{Ice around star-forming regions: a galactic and extra- galactic perspective \\ J.V. Keane $\bullet$ Kapteyn Institute, A.G.G.M. Tielens $\bullet$ Kapteyn Institute,} H.W.W. Spoon $\bullet$ Kapteyn Institute

The origin of life is intimately interwoven with the molecular material associated with the birth and evolution of stars, planetary systems and the interstellar medium. In such environments ice mantles form on the dust surfaces and it is now believed that these are the dominant reservoirs of biogenic elements in molecular clouds. Moreover, observations have revealed a push towards molecular complexity driven by grain surface reactions - forming ices and their release into the gas-phase. In the outer parts of budding elements of planetary systems, these ice grains are the progenitors of cometary bodies which can later deliver volatiles to inner Earth-like planets. The launch of the Infrared Space Observatory (ISO) provided a revolution in our understanding of the nature of these icy dust particles. To date interstellar features of $\mathrm{H}_{2} \mathrm{O}$, $\mathrm{CO}, \mathrm{CO}_{2}, \mathrm{CH}_{3} \mathrm{OH}, \mathrm{CH}_{4}, \mathrm{H}_{2} \mathrm{CO}, \mathrm{HCOOH}$ and $\mathrm{OCS}$ as well as other minor species are observed. In addition, ISO is helping to ascertain the composition of the cold dusty material residing in galaxies beyond our own Milky Way. Therefore, a unique comparison of solar, galactic and extra-galactic material is now possible and there are tantalizing indications of the similar nature of dust in our galaxy to that in others. Here, recent data on a variety of characteristic species along with a comparison between galactic and extra-galactic targets will be presented. 


\section{Delivery of organic carbon to Earth by large, strongly heated micrometeorites}

Monika Kress $\bullet$ University of Washington, Don Brownlee $\bullet$ University of Washington, Dave Joswiak $\bullet$ University of Washington

Most of the extraterrestrial carbon accreted by Earth is carried by the 40000 tons of $\sim 0.2 \mathrm{~mm}$ micrometeorites that enter the atmosphere every year. Particles in this size range would have supplied an enormous amount of carbon to the incipient biosphere since the exogenous influx at $\sim 4 \mathrm{Ga}$ would have been much higher than today. However, these particles undergo strong drag heating to $\sim 1500 \mathrm{~K}$ for several seconds upon atmospheric entry, ostensibly burning away all the organics as CO. Ironically, particles of this size seemed to contribute no organic carbon to Earth despite the fact that they constitute the bulk of the total incoming mass. Conventional wisdom has thus held that organics survive only in smaller cosmic dust grains and in $>\sim \mathrm{cm}$-sized meteorites, which account for only a tiny fraction of the total exogenous mass flux.

However, carbon has been found in several smaller, yet still strongly heated particles in the form of a refractory, char-like material imbedded with tiny FeNi metal beads, motivating us to model the pyrolysis of complex organic matter. Our results suggest that the transient nature of the drag heating of micrometeorites produces small aromatics, light hydrocarbons, and other organics, as well as $\mathrm{CO}$ and char, indicating that strongly heated micrometeorites may have indeed been a significant source of organic carbon during the origin of life on Earth.

\section{The structure of matter and the germs of life \\ Paul Lauterbur $\bullet$ University of Illinois, Urbana-Champaign}

The origin of life may have depended upon some robust hithertounappreciated deep principles of chemical self-organization. There has been much speculation about the possibility of an intermediate stage that may have involved, for example, adsorption on mineral surfaces. But there is another possible starting point, hinted at by the phenomenon of molecular imprinting. A crosslinked matrix of silica or organic polymer, formed in the presence of molecules not linked into the network, retains a special affinity for those or very similar molecules (but not their optical isomers) after the original imprinting molecules have been washed out, and also has catalytic activity. It can be speculated that similar but evanescent structures are formed in liquids and solids near the glass transition, extending the generality of the phenomenon. Such structures could be preserved in geological formations, carbonaceous chondrites, and astronomical bodies, where new methods of examination would be required to detect and characterize them. They may also be re-created in the laboratory under controllable conditions, using simple polymers or the "multimers" from prebiotic chemistry experiments. Specific binding and catalytic activity must have been present in matter before the rise of biology, and may have contributed to that event.

\section{ATP-dependent DNA enzymes}

Matthew Levy $\bullet$ University of Texas, Austin, Andrew D. Ellington $\bullet$ University of Texas, Austin

In an effort to bridge the gap between simple nucleic acid replicating systems and complex ribozymes which would be required for an RNA world, we have been investigating the ability of small molecules to modulate the ligation of oligonucleotides.

We have selected deoxyribozymes that catalyze a ligation reaction largely via a templating mechanism. In this respect, our catalysts are more akin to the simple ligators of von Kiederowski, and less like other nucleic acid catalysis that require the formation of a catalytic core.

Effector-activated ribozymes have previously been generated by appending binding species (aptamers) to ribozymes. In order to determine whether our deoxyribozymes could be activated by effector molecules, we have appended an anti-adenosine aptamer to our deoxyribozyme ligase. The resultant constructs are specifically activated up to 460 -fold by ATP. Surprisingly, effect or activation is achieved by suppressing the rate of the background, templated ligation reaction in the absence of the effector molecule, probably by misalignment of the oligonucleotide substrates. This novel allosteric mechanism has not previously been observed for nucleic acid catalysts.

These results further support the idea that small molecules can modulate the interactions of nucleic acids. In addition they provide a means for the design and study of effector-dependent cyclic networks.

\section{FTIR and STXM detection of organic carbon in Antarctic micrometeorites}

Graciela Matrajt $\bullet$ CSNSM, George Flynn $\bullet$ State University of New York, Plattsburgh, Michel Maurette $\bullet$ CSNSM

Antarctic micrometeorites (AMMs), large interplanetary dust particles, should have contributed to the delivery of the building blocks necessary for the origin of life on the early Earth. We have analysed three AMMs and a terrestrial particle collected and curated just as the AMMs studied. We have also studied a Murchison fragment used as a standard. The analyses were performed first by FTIR (Fourier transform infrared) spectroscopy which indicates the presence of aliphatic hydrocarbons, and by STXM (scanning transmission X-ray microscope) spectroscopy with two modes: stack-imaging mode to map the distribution of carbon over a region of the section, and the C-XANES (carbon-X-ray absorption near edge structure) mode which allows the determination of the $\mathrm{C}$ bonding states at selected spots. FTIR characterization: the terrestrial control particle did not show any absorption in the $\mathrm{C}-\mathrm{H}$ stretching region. The features detected in AMMs and Murchison exhibit the same aliphatic hydrocarbon absorption peaks. STXM characterization: the terrestrial particle did not show any peak over noise signature. The AMMs exhibited, such as Murchison, C-ring structure and carbonyl functional group features. Conclusion: these same features are also found in some IDPs, suggesting that this carbon is indigenous and thus extraterrestrial.

\section{Interior fluids in asteroids and meteorites: electrolytes, nutrients, and biological responses: implications for biogenesis and panspermia in solar nebulae}

Michael N. Mautner $\bullet$ Lincoln University, Lincoln, New Zealand, C.L. Apel • University of California, Santa Cruz

Extracts of carbonaceous chondrite meteorites in planetary microcosms simulate fluids in asteroids and comets. The solutions reach concentrations of $3.8 \mathrm{~mol} / 1$ electrolytes and about $0.1 \mathrm{~mol} / 1$ organics. Similar solutions trapped in asteroids during aqueous alteration, and in meteorites on aqueous planets, can form complex biomolecules, including vesicle-forming membranes. Inoculated with microorganisms, these internal solutions were observed to sustain $4 \times 10^{5} \mathrm{CFU} / \mathrm{ml}$ algae and $6 \times 10^{6} \mathrm{CFU} / \mathrm{ml}$ bacteria over long periods. Collisions between asteroids containing such solutions during aqueous alteration can disperse microorganisms in solar nebulae and transport them to planets and to other solar systems. The extractable nutrients in the carbonaceous asteroids can sustain a biomass of $10^{18} \mathrm{~kg}$ in $10^{32}$ microorganisms. The total organics in asteroids and comets are sufficient to seed all new solar systems in the galaxy with microorganisms. The results suggest that protoplanetary nebulae and asteroids/meteorites may be conducive to the origins of life, to early evolution, and to seeding the galaxy by natural or directed panspermia.

\section{References}

Mautner (2002). Asteroid solutions Astrobiology 2 in press.

Apel et al. (2002). Membranes, BBA 1559, 1-9.

Mautner (1997). Panspermia, J. British Interplanetary Soc. 45, 653.

E-file at http://www.panspermia-society.com

\section{Jigsaw model of the origin of life \\ John McGowan $\bullet$ GFT Group Incorporated}

It is suggested that life originated in a three-step process referred to as the jigsaw model. RNA, proteins, or similar organic molecules polymerized in a dehydrated carbon-rich environment, on surfaces in a carbon-rich environment, or in another environment where polymerization occurs. These polymers subsequently entered an aqueous environment where they folded into compact structures. It is argued 
that the folding of randomly generated polymers in water tends to partition the folded polymer into domains with hydrophobic cores and matching shapes to minimize energy. In the aqueous environment, hydrolysis or other reactions fragmented the compact structures into two or more matching molecules, occasionally producing simple living systems. It is argued that the hydrolysis of folded polymers such as RNA or proteins is not random. The hydrophobic cores of the domains are rarely bisected due to the energy requirements in water. Hydrolysis preferentially fragments the folded polymers into pieces with complementary structures and chemical affinities. Thus the probability of synthesizing a system of matched, interacting molecules in prebiotic chemistry is much higher than usually estimated. Environments where this process may occur are identified. The implications of this hypothesis for seeking life or prebiotic chemistry in the Solar System and the laboratory are explored.

\section{The origin of organic matter in the solar system: evidence from the interplanetary dust particles} John McGowan $\bullet$ GFT Group Incorporated, George Flynn $\bullet$ State University of New York, Plattsburgh, Lindsay P. Keller $\bullet$ NASA Johnson Space Center, Chris Jacobsen $\bullet$ State University of New York, Stony Brook, Sue Wirick • State University of New York, Stony Brook The origin of organic matter in interplanetary material has not been established. A variety of processes have been proposed. Two extreme cases are a Fisher-Tropsch process operating in the gas phase of the solar nebula (or the interstellar medium) or a Miller-Urey process, which requires interaction with liquid water, presumably on an asteroid. To infer the origin of organic matter in the Solar System we measured the abundance and types of carbon in all three kinds of primitive (not thermally processed) extraterrestrial materials available for laboratory analysis: anhydrous IDPs, hydrated IDPs, and hydrated carbonaceous chondrite meteorites. We employed two synchrotron-based instruments to examine 12 IDPs and four carbonaceous meteorites (Orgueil, Tagish Lake, Murchison and Murray: (1) a scanning transmission X-ray microscope, to map the $\mathrm{C}$ distribution, measure the $\mathrm{C}$ abundance, and perform C X-ray absorption near-edge structure (XANES) spectroscopy, to identify the carbon functional groups, and (2) a Fourier transform infrared (FTIR) spectrometer, to identify the functional groups of organic compounds. The similarity of the $\mathrm{C}$ abundance, CXANES and FTIR spectra of anhydrous IDPs, hydrated IDPs, and hydrated carbonaceous meteorites is consistent with the production of much of the organic matter prior to incorporation into asteroids, possibly by irradiation of C-bearing ices or by a Fisher-Tropsch-type process operating in the gas phase of the nebula or the interstellar medium (given large D excess in some IDPs).

\section{Surface autocatalytic HCN polymerization, evidence for glycine oligomers in $(\mathrm{HCN}) \mathrm{X}$ and a water-soluble $\mathrm{HCN}$ hexamer}

Robert Minard $\bullet$ Pennsylvania State University, Karma Parsons $\bullet$ Syracuse University, Anna Johnson • Gannon University, Erin Shields • Pennsylvania State University, Marc Fiddler $\bullet$ Pennsylvania State University, A. Daniel Jones $\bullet$ Pennsylvania State University, Clifford Mathews $\bullet$ University of Illinois, Chicago

$\mathrm{HCN}$ polymerization is autocatalytic. When a solution of $\mathrm{HCN}$ in toluene is seeded with minute quantities of solid $\mathrm{HCN}$ polymer, the particles catalyze the formation of fresh $\mathrm{HCN}$ polymer on their surface. At room temperature, almost all of the $\mathrm{HCN}$ has polymerized onto the expanding particle surface to form a large solid mass within 10 days.

Using a degradation-derivatization method (TMAH thermochemolysis/GC-MS), diketopiperazine is formed from di- and tri-glycine, but not glycine itself. $\mathrm{HCN}$ polymer also produces substantial quantities of the same diketopiperazine indicating it contains glycyl-glycyl or (glycyl) $\times$ substructures.

Water-soluble extracts of $\mathrm{HCN}$ polymer yield a number of products including urea and a new compound with formula $\mathrm{C}_{6} \mathrm{H}_{8} \mathrm{~N}_{6}$. Using NMR and ESI-MS-MS, it has been assigned the structure 2-cyano-3, 4, 5, 6-tetraaminopyridine, with a reasonable mechanism of formation based on known cyanide condensation chemistry.
Fresh water or marine environments for the earliest forms of life? Part II: non-enzymatic polymerization of early genetic material

Pierre-Alain Monnard $\bullet$ University of California, Santa Cruz, Anastassia Kanavarioti $\bullet$ University of California, Santa Cruz, David W. Deamer $\bullet$ University of California, Santa Cruz

The RNA-world hypothesis requires an efficient non-enzymatic polymerization of RNA from a probably dilute monomer solution. Using eutectic phases in ice at $-18{ }^{\circ} \mathrm{C}$ to concentrate dilute phosphoimidazoleactivated single monomer (ImpN) solutions or mixtures containing the four nucleobases at low initial ionic strength, we demonstrated that the formation of random oligomer fragments as long as 14 mers occurred with a monomer incorporation of over $80 \%$. These results suggest that synthetic reactions could take place in a prebiotic ice matrix without requiring transient melts produced by large cometary or meteoritic impacts. $^{1}$

We further studied the effect of an initial, high ionic strength medium on RNA polymerization, and found that this reaction is markedly inhibited by salt concentrations much lower than those in today's oceans. Under these conditions, the main reaction products were pyrophosphate dimers. An early ocean would presumably contain substantial concentrations of inorganic ions such as $\mathrm{Na}^{+}, \mathrm{Cl}^{-}, \mathrm{Mg}_{2}^{+}$, $\mathrm{Ca}_{2}^{+}$and $\mathrm{Fe}_{2}^{+}$. Such an environment would have not been suitable for a nonenzymatic polymerization of early genetic material. More appropriate environments would have been ponds and lakes of fresh water produced by the accumulation of precipitation on island-type landmasses.

References

${ }^{1}$ Bada, J.L., Bigham, C. \& Miller, S.L. (1994). Proc. Nat. Acad. Sci. USA 91, 1248.

Who or what? Replication of identities or functions Michael New $\bullet$ NASA, Charles Blackwell $\bullet$ Dynamic Systems Consultants, Kagan Tumer $\bullet$ NASA, Dimitris Stassinopoulos $\bullet$ University of California, San Francisco

In this presentation, we will present results on the fundamental properties of two classes of replicating systems: autocatalytic replicators that reproduce exact copies of a template molecule, and function-reproducers that maintain a set of essential functions without replicating the identities of the functional moieties. We will describe the stability and behavior in-the-large of autocatalytic replicators. Most importantly, we have found no sharp distinction between an autocatalytic and a nonautocatalytic domain. We will also present a new derivation of von Kiedrowski's square-root rate law. Function-reproducers are proposed as an important component of protocells and we will present theoretical results on a simple model system that incorporates known peptide biophysics. For a wide range of parameters, we have shown that this type of system can improve its overall performance, even in the absence of any method for information storage. This type of system improvement is defined to be non-genomic evolution.

\section{Multiscale simulation of molecular self-assembly}

Martin Nilsson • Los Alamos National Laboratory, Steen Rasmussen • Los Alamos National Laboratory, Donatella Pasqulini $\bullet$ Los Alamos National Laboratory

We are developing a framework consisting of a linked hierarchy of different simulation techniques, suited for simulating molecular selfassembly processes. The techniques are: molecular dynamics (MD), molecular dynamics lattice gas, and lattice Boltzmann. This framework enables up- and downscaling between Angstroms and millimeters and femtoseconds and minutes. Understanding self-assembly processes is essential to map possible pathways for the origin of life.

In the MD lattice gas, molecules are spatially confined to a threedimensional lattice, whereas the momentum space is continuous. The translational movements are probabilistic. Collisions locally preserve momentum, angular momentum and kinetic energy. The particles interact via continuous fields. Kinetic and potential energy can be exchanged, resulting in non-trivial thermodynamics. We have used this technique to study spontaneous micellation of amphiphilic polymers in 
water. The simulations start from a random initial distribution but spontaneously evolve to steady states of growing and dividing micelles.

\section{The organic refractory material in the diffuse interstellar medium: mid-IR spectroscopic constraints \\ Y.J. Pendleton $\bullet$ NASA Ames Research Center, L.J. Allamandola $\bullet$ \\ NASA Ames Research Center}

We present an analysis of the $4000-1000 \mathrm{~cm}^{-1}$ (2.5-10 micron) region of the spectrum of diffuse interstellar medium (DISM) dust compared with the spectra of 13 chemical entities produced in the laboratory that serve as analogs to the interstellar material. The organic signatures of extragalactic dust, carbonaceous chondritic material, and E. coli bacteria are also presented because these have been discussed in the literature as being relevant to the diffuse interstellar medium. Spectral analysis of the DISM allows us to place significant constraints on the applicability of proposed candidate materials. The spectra of candidate materials are evaluated using four spectral characteristics based on the interstellar data: (i) comparisons of the profile and sub-peak positions of the $2940 \mathrm{~cm}^{-1}$ (3.4 micron) aliphatic $\mathrm{CH}$ stretching-mode band, (ii) the ratio of the optical depth (O.D.) of the aliphatic $\mathrm{CH}$ stretch to the O.D. of the $\mathrm{OH}$ stretch near $3200 \mathrm{~cm}^{-1}$ (3.1 micron), (iii) the ratio of the O.D. of the aliphatic $\mathrm{CH}$ stretch to the O.D. of the carbonyl band near $1700 \mathrm{~cm}^{-1}$ (5.9 micron), and (iv) the ratio of the O.D. of the aliphatic $\mathrm{CH}$ stretch feature to the O.D. of the $\mathrm{CH}$ deformation modes near $1470 \mathrm{~cm}^{-1}$ (6.8 micron) and $1370 \mathrm{~cm}^{-1}$ (7.25 micron).

We conclude that the organic refractory material in the diffuse interstellar medium is predominantly hydrocarbon in nature, possessing little nitrogen or oxygen, with the carbon distributed between the aromatic and aliphatic forms. Long alkane chains $\mathrm{H}_{3} \mathrm{C}^{-}\left(\mathrm{CH}_{2}\right)_{n}^{-}$with $n$ much greater than 4 or 5 are not major constituents of this material. Comparisons to laboratory analogs indicate that the DISM organic material resembles plasma-processed pure hydrocarbon residues much more so than energetically processed ice residues. This result is consistent with a birth site for the carrier of the 3.4 micron band in the outflow region of evolved carbon stars. The organic material extracted from the Murchison carbonaceous meteorite and the spectrum of E. coli bacteria reveal spectral features in the 5-10 micron region that are absent in the DISM. Although the presence of unaltered circumstellar components in the Murchison meteorite has been established through several lines of evidence, it is unclear whether or not the aliphatic component which gives rise to the 3.4 micron band is in that category. Considering the complete 2-10 micron wavelength region, there is no spectral evidence for a biological origin of the 3.4 micron interstellar absorption band. The similarity of the aliphatic $\mathrm{CH}$ stretch region of dust from our own galaxy compared with that of distant galaxies suggests that the organic component of the ISM is widespread and may be an important universal reservoir of prebiotic organic carbon.

\section{Surface electrochemistry, pre-biotic chemistry and the mineral origin of life}

Michael Philpott $\bullet$ National University of Singapore, Ming W. Wong • National University of Singapore, Ting T. Lin $\bullet$ Institute of Materials Research and Engineering, Hui C. Yeo $\bullet$ National University of Singapore, Igor Y. Goliney $\bullet$ National University of Singapore Basic issues in mineral surface origin of life on Earth hypotheses: (i) how free energy of mineral reactions drives reductions (starting from $\mathrm{CO}_{2}$ ) or oxidations (starting from $\mathrm{CH}_{4}$ ) to the $\{\mathrm{Cl}\}$ organic manifold; (ii) what surface structures catalyze reactions leading to anabolic metabolic assemblies containing $\left\{\mathbf{C}_{n}(n>1)\right\}$ manifolds. This area is nearly impossible to explore experimentally because of high pressure, temperature and flow of aqueous fluids associated with tectonic activity of the early Earth. Our strategy to study key issues left unanswered in the iron-sulfur and clay mineral scenarios is to use $a b$ initio quantum chemistry, molecular dynamics (MD) and Monte Carlo simulations. These are powerful tools for exploring properties and reaction mechanisms in extreme physical conditions. Current work focuses on iron sulfide systems. We are calculating the equilibrium geometry and electronic structure of a series of clusters representing the minerals FeS, $\mathrm{Fe}^{1-x} \mathrm{~S}(0<x<0.125), \mathrm{FeS}_{2}$ including the adsorption of small neutrals and ions $\left(\mathrm{H}_{2} \mathrm{O}, \mathrm{H}_{2} \mathrm{~S}, \mathrm{OH}^{-}, \mathrm{SH}^{-}, \mathrm{Cl}^{-}, \mathrm{H}^{+}, \mathrm{Na}^{+}\right)$. Our goal is the identification of plausible reaction partners and pathways for anabolic synthesis. Another is the determination of parameters for larger semiempirical electronic calculations and interaction potentials for MD simulations of the aqueous electric double layers of charged minerals.

\section{Search for organic matter in Leonid meteoroids}

Rick Rairden • Lockheed Martin, Peter Jenniskens • SETI Institute Near-ultraviolet spectra $(320-410 \mathrm{~nm})$ of Leonid meteors were obtained in an effort to measure the strong $\mathrm{B} \rightarrow \mathrm{X}$ emission band of the radical $\mathrm{CN}$ at $387 \mathrm{~nm}$. CN is an expected product of ablation of nitrogencontaining organic carbon in the meteoroids as well as a possible product of the aerothermochemistry induced by the meteor's kinetic energy. The meteor spectra were recorded in the pre-dawn hours of November 18 with an objective grating (slit-less) spectrograph deployed on the FISTA aircraft during the 2001 Leonid Multi-Instrument Aircraft Campaign (MAC). A lower-resolution version of this instrument was flown on the 1999 Leonid MAC, where the spectra were found to be dominated by neutral iron lines with no clear signature of the $\mathrm{CN}$ band. Our new higher-resolution spectra will be presented, with improved upper limits on the possible $\mathrm{CN}$ content.

\section{A comprehensive study of the effect of coronae processes in Titan's troposphere}

Sandra I. Ramirez $\bullet U N A M$, Rafael Navarro-Gonzalez $\bullet U N A M$

In order to understand the influence of different operational parameters of corona discharges on the energy yields of organic compounds in Titan's simulated troposphere, mixtures containing methane in nitrogen were subjected to the action of positive and negative polarity coronas using a coaxial configuration. The methane content in the mixtures varied from 2 to $10 \%$ according to the latest estimations for this molecule in the satellite's atmosphere. An increment in the hydrocarbon production was determined at the highest carbon content. Methane clouds have been proposed to exist between 10 and $30 \mathrm{~km}$ altitude. We have experimentally varied the initial pressure from 0.1 to $1 \mathrm{bar}$, which resulted in a steady production of hydrocarbons and nitriles for most of the cases. One of the most intriguing questions about Titan is related to the electrical activity in its atmosphere. A change in the current density and in the initial values of electrical power delivered to the simulated troposphere resulted in differences in the type and amount of compounds formed. Analytical quantification was performed by a coupled GCMS-FTIR system. These results are expected to shed some light on the complexity and prebiotic relevance of organic chemicals in the outer Solar System and to help in the interpretation of the information collected by the Cassini-Huygens mission.

\section{Increasing the catalytic potential of nucleic acids}

Alonso Ricardo $\bullet$ University of Florida, Matthew Carrigan $\bullet$ University of Florida, Steven Benner $\bullet$ University of Florida

In vitro selection (IVS) experiments suggest that the enzymatic activity of nucleic acids is rare and the catalytic power is weaker in comparison to proteins. Therefore, the addition of the functionality found in proteins to random libraries of nucleic acids is expected to increase the diversity and efficiency of catalyzed reactions. So far this approach has failed to dramatically improve catalytic power. This suggests that either the protocols used to select for catalytic molecules or in the extreme case our chemical understanding of the system is flawed.

A possible explanation is that under typical IVS experiments, molecules that exhibit high catalytic efficiency ("super-catalysts") are being lost during sample preparation before the actual selection procedure is started

We have performed several IVS experiments (with standard and additional functionality) using a photo-deprotectable ribose linker to prevent the loss of "super-catalysts" during initial setup and under increasing selection time stringency. This approach offers us the possi- 
bility of understanding the origins and evolution of catalysis by estimating populations distributions during IVS experiments and generating a model of landscape space that describes the distribution of catalytic power and the "evolvability" of random populations.

\section{How many protein ancestors, and of what kind?} Monica Riley • Marine Biological Laboratory, Laila Nahum $\bullet$ Marine Biological Laboratory, Sulip Goswami $\bullet$ Marine Biological Laboratory The (hypothetical) last common ancestor to the three domains of the tree of life contained a large diversity of proteins, similar to those found today in common in organisms of all three domains of life. The development of hundreds, even thousands, of individual proteins of specific biological function had already taken place by the time an early cell-like entity had evolved. To get some insight into the origins of differentiated proteins in the period preceding the assembly of the first cell, we can try to estimate how many and what kinds of ancestral proteins would have been required to generate the observed diversity.

Proteins can be grouped into related superfamilies by several means. Amino acid sequence similarity is one property that collects related proteins into groups. The similarity of structural elements and overall tertiary configuration are others. Since there is so much known about E. coli proteins, we have chosen this set to analyze. We will present information about the numbers of sequence similar groups, their sizes and their functions. Work on structural classes has begun. Over 2000 of E. coli's 4407 proteins have known folds (structural domains). These proteins have been grouped according to their domain content into 55 classes. Further, information on tertiary configuration of the proteins can be gained through so-called "threading" algorithms that map an unknown protein onto a related experimentally known one. Data will be presented. Since sequence-related families and structurally related families will not be identical, analysis of the relationships between them focuses on plausible mechanisms of protein evolution.

\section{The rapid exoergic oligomerization of amino acids} David S. Ross • United States Geological Survey, Kono H. Lemke • Stanford University, James L. Bischoff $\bullet$ United States Geological Survey

Prebiotic assembly of amino acids and nucleotide components to functioning biomolecules required the generation of amide and ester links. Their formation in an aqueous environment is energetically uphill, and their creation would appear to override thermodynamic principles. Recent observations by Porter (1998) and Matsuno (2000) of glycine oligomerization on mineral surfaces and in hydrothermal environments, respectively, suggest that consolidation takes place, apparent thermochemistry notwithstanding. Moreover the condensation rates are clearly competitive with decarboxylation, a deletory reaction path obviously not leading to developing biochemistry. In gold bag studies in liquid water at $260{ }^{\circ} \mathrm{C}$ we find up to $75 \%$ of the amino acid charge vanishes rapidly in a first order process at a rate about 30 times that of decarboxylation, the route followed by the remaining acid. Polyglycines develop during this initial period at rates far more rapid than estimates developed from the known thermochemistry and the rates of hydrolysis. A peptide synthesis scheme emerges from these observations that includes; (1) rapid sorption at surface sites, (2) exoergic growth due to b-sheet-like geometry, and (3) desorption through dissolution of the mineral underbed. Since oligomer solubilities fall by a factor of four to five for each added unit, hydrolysis becomes increasingly less significant with size.

\section{The strictly endogenous origins of amino acids: a chemical kinetics test \\ David S. Ross • United States Geological Survey, Kono H. Lemke • Stanford University, James L. Bischoff • United States Geological Survey}

A fully endogenous path to life from $\mathrm{CO}_{2} / \mathrm{N}_{2} / \mathrm{H}_{2} \mathrm{O}$ on the early Earth had to have overcome several challenges, the most severe of which was probably satisfactory competitive chemical kinetics. The essential requirements for life's precursors are reactions providing $\mathrm{C}-\mathrm{C}$ and $\mathrm{C}-\mathrm{N}$ bonds, polyfunctionality, and branching carbon chains. These requisites in turn ordain temperatures no greater than about $100{ }^{\circ} \mathrm{C}$. With a reduction potential provided by FMQ and PPM it can be shown that the $\mathrm{HCN} /$ formaldehyde route to amino acids is not supported. Similar barriers emerge for nucleotides. Thermodynamics dictates the ultimate state to be $\mathrm{CH}_{4} / \mathrm{NH}_{3} / \mathrm{H}_{2} \mathrm{O}$, and thus metastable equilibria are required. But a mere pause in the march to the final composition is not sufficient. The cardinal need in a successful system are routes to biosignificant molecules that are kinetically competitive with both further reduction and decarboxylation. The reductive sequences $\mathrm{N}_{2} \rightarrow \mathrm{NH}_{3}$ and $\mathrm{CO}_{2} \rightarrow \mathrm{HO}_{2} \mathrm{CCO}_{2} \mathrm{H} \rightarrow \mathrm{CHOCO}_{2} \mathrm{H}$ are thermodynamically acceptable and are reported in laboratory studies, and the reductive amination of $\mathrm{CHOCO}_{2} \mathrm{H}$ to glycine has been demonstrated. Analysis shows, however, that in natural settings the chain cannot proceed beyond $\mathrm{HO}_{2} \mathrm{CCO}_{2} \mathrm{H}$ since decarboxylation rates will exceed those of reduction. On this basis we suggest that life could not have evolved from that starting point, and outside assistance was required.

\section{A possible prebiotic synthesis of purine, adenine, cytosine, and $4(3 \mathrm{H})$-pyrimidinone from formamide: implications for the origin of life}

Raffaele Saladino • University della Tuscia, Viterbo, Italy, Claudia Crestini $\bullet$ University di Roma Tor Vergata, Roma, Italy, Giovanna Costanzo $\bullet$ Centro di Studio per gli Acidi Nucleici, CNR, Roma, Italy, Rodolfo Negri • University La Sapienza, Roma, Italy, Ernesto Di Mauro • University di Roma La Sapienza, Roma, Italy

$\mathrm{HCN}$ chemistry provides a route for the prebiotic synthesis of purines and pyrimidines. In addition to the condensation into nucleobases, HCN hydrolyses to formamide, a possible additional down the-line candidate for the synthesis of nucleobases. We report on a plausible prebiotic synthesis of naturally occurring purine and pyrimidine derivatives from formamide under catalytic conditions. In the presence of catalysts formamide leads to the formation of purine, adenine, cytosine, and $4(3 \mathrm{H})$-pyrimidinone. The role of catalysts is also relevant in providing a high selectivity in the products distribution. A circular reaction involving synthesis and degradation of purines and pyrimidines based on formamide chemistry has been identified, making plausible an important role of formamide in prebiotic bio-oriented chemistry. The role of a specific catalyst in the selectivity of pre-biotic reactions is demonstrated by the sequence-selective degradation of oligonucleotides. This finding links the formamide-based cyclic synthetic-degradative reactions with the onset of Darwinian molecular evolution, potentially leading to self-replicating structures. All the compounds involved are present in extraterrestrial environments.

References

See: Bioorg. Med. Chem. 9, 1249, 2001, commented in TIBS, 26, 153, 2001 and J. Am. Chem. Soc. 1996, 118, 5615; Bioorg. Med. Chem. 1997, 5, 2041.

\section{Building an RNA world, piece by piece}

Frank Schmidt $\bullet$ University of Missouri-Columbia

Large ribozymes, like their protein counterparts, consist of a relatively small active site surrounded by secondary and tertiary structures that enhance catalysis. Although large ribozymes can be selected from combinatorial libraries in vitro, it is unlikely that this situation held in the absence of an efficient replicator. The relatively low repetitive yield of nucleotide condensation by solution chemistry means that the concentration of RNAs on the order of $100 \mathrm{nt}$ would be vanishingly small in a prebiotic situation. We propose instead that large ribozymes would be derived through modular assembly, a process we have termed accretion. As a test of the likelihood of the accretion model, we have deleted a small structural module from RNase P RNA and characterized the effect of the deletion on enzyme activity. We determined the solution structure of the module and its functional requirements for its function in RNase P activation. The information content of the module can be related to the plausibility of accretion as an in vitro evolutionary mechanism. 
Evaluating organic contamination in hydrothermal prebiotic synthesis experiments

Alexander Smirnov $\bullet$ State University of New York, Stony Brook, Martin A.A. Schoonen $\bullet$ State University of New York, Stony Brook Prebiotic synthesis experiments often yield organic compounds in low concentrations. Hence, it is important to conduct blank experiments to determine contaminant levels. As part of a study into the stability/ reactivity of dissolved formate we conducted blank experiments with a 316 ss reaction vessel and a $6 \mathrm{Al} / 4 \mathrm{~V}$ Ti vessel, both equipped with quartz glass inserts. Various materials for sampling tubes and for thermocouple sheaths were tested. Stirring was performed using an orbital shaker or motorized internal Teflon stirrer. All experiments were conducted using DI UF/UV water in a closed system with a $\mathrm{N}_{2}$ headspace $(\sim 400 \mathrm{psi})$. Experiments lasted for 7 to 19 days at $150-165^{\circ} \mathrm{C}$. Samples were analyzed using ion chromatography. Besides formate, analyses also showed the presence of acetate and propionate ions at $\mu \mathrm{M}$ levels.

\begin{tabular}{|c|c|c|c|c|}
\hline Vessel & Tubing & Stirring & $\begin{array}{l}\text { Thermocouple } \\
\text { sheath }\end{array}$ & $\begin{array}{l}\text { Formate } \\
{[\mu \mathrm{M}]}\end{array}$ \\
\hline $316 \mathrm{SS}$ & PTFE & TFE & $\begin{array}{l}\text { PTFE heat } \\
\text { shrink }\end{array}$ & $\sim 40$ \\
\hline 6AL/4V Ti & $\begin{array}{l}\text { Gr-2 Ti, } \\
\text { PEEK }\end{array}$ & No stirring & $\begin{array}{l}\text { PTFE heat } \\
\text { shrink }\end{array}$ & $\sim 13$ \\
\hline 6AL/4V Ti & $\begin{array}{c}\text { Gr-2 Ti, } \\
\text { PEEK }\end{array}$ & Orb. Shaking & Gr-2 Ti & $\sim 5$ \\
\hline $6 \mathrm{AL} / 4 \mathrm{~V} \mathrm{Ti}$ & Gr-2 Ti & Orb. Shaking & Gr-2 $\mathrm{Ti}$ & $\sim 1$ \\
\hline
\end{tabular}

From our experiments, we conclude that the most suitable material for hydrothermal experiments is titanium covered with a Ti-oxide layer. Polymer tubing, including PEEK, introduces contamination when heated to $150^{\circ} \mathrm{C}$ for several days.

\section{Influence of seawater sulfate concentrations on the chemistry of hydrothermal vent solutions: a modeling approach \\ Alexander Smirnov • State University of New York, Stony Brook,} Martin A A. Schoonen $\bullet$ State University of New York, Stony Brook The objective is to evaluate how the composition of solutions discharging from submarine hydrothermal vents depends on the seawater (sw) composition. Here we focus on the effect of sw sulfate concentrations, which may have been lower in ancient sw and limit the amount of $\mathrm{H}_{2} \mathrm{~S}$ that can discharge from these systems. Constraints on $\mathrm{H}_{2} \mathrm{~S}$ in vent solutions are important because it is probably the most reactive reductant in these systems. The research strategy we have employed here is to use reaction path modeling to simulate the evolution of sw free of $\mathrm{O}_{2}$ with varying amounts of sulfate as it circulates through a subseafloor hydrothermal systems at mid-oceanic ridges. The reaction path of sw with $10 \%, 25 \%, 50 \%, 75 \%$, and $100 \%$ of present-day sulfate concentration was calculated with four different mineral buffers (IW, PPM, QFM, HM). Minerals were allowed to precipitate from solution in this model that simulates the sw up to $300{ }^{\circ} \mathrm{C}$. In order to simulate the sluggish kinetics of thermochemical sulfate reduction, we evaluated under each condition the effect of heating the sw to $200^{\circ} \mathrm{C}$ without attaining redox equilibrium with the mineral buffer. The results indicate that the $\mathrm{H}_{2} \mathrm{~S}$ content in discharging solution is controlled by pyrrhotite solubility and independent of the initial sulfate concentration.

\section{Reaction kinetic analysis of the assembly and the dynamics of a proto-organism}

Konbjoern Tunestroem $\bullet$ Los Alamos National Laboratory, University of Trondheim, NTU Norway, Steen Rasmussen $\bullet$ Los Alamos National Laboratory, Santa Fe Institute, Nils A. Baas • University of Trondheim, NTU Norway, John S. McCaskill • Fraunhofer Society BioMIP, Sankt Augustin, Germany

As part of the project to assemble an artificial proto-organism at Los Alamos, we examine the dynamics of the involved physicochemical process in terms of their mass action kinetics. According to our definition of a proto-cell these components consist of a proto-compartment, a proto-metabolism, and proto-genes. Initially the sub-components of the proto-organism are analyzed separately.

(i) Assembly dynamics and equilibrium size distribution of micellar and vesicle structures are expressed using Markov chains, which in the continuum limit results in a partial differential equation, which we have examined and solved in special cases. (ii) The dynamics of coupled redox reactions between the water and lipid phase, assumed to define a proto-metabolism, are analyzed and compared with the corresponding experimental kinetics. (iii) Assuming that a proto-gene system can be implemented as a replicating polymer with binary ligation, where the ligating fragments can also react with each other, introduces competition for the replication building material. The dynamics of this and other replicater systems are analyzed. (iv) Finally an integrated reaction kinetic model of the full proto-organism is presented and analyzed numerically for rate-limiting reaction steps and alternative coupling reactions using reaction constants from the experimental literature. Sensitivity analysis is done for crucial reaction constants, which have not yet been experimentally determined.

\section{Abiogenic and biogenic graphite in the $3.8 \mathrm{Ga}$ Isua Supracrustal Belt}

Mark van Zuilen $\bullet$ Scripps Institution of Oceanography, Aivo Lepland $\bullet$ Geological Survey of Norway, Gustaf Arrhenius $\bullet$ Scripps Institution of Oceanography

The principal method for studying the earliest traces of life in the metamorphosed, oldest $(>3.5 \mathrm{Ga})$ terrestrial rocks involves determination of the isotopic composition of carbon, mainly prevailing as graphite. Here we show that most graphite in the $3.8 \mathrm{Ga}$ old Isua Supracrustal Belt (ISB) occurs in carbonate-rich metasomatic rocks (metacarbonates) while sedimentary units, including BIFs and metacherts, have exceedingly low graphite concentrations. Thermal decomposition of siderite; $6 \mathrm{FeCO}_{3}=2 \mathrm{Fe}_{3} \mathrm{O}_{4}+5 \mathrm{CO}_{2}+\mathrm{C}$, is the process seemingly responsible for the graphite formation in these metacarbonates. The siderite-graphite-apatite association in the ISB consequently appears to be an entirely abiogenic metasomatic feature, which does not point to traces of ancient life. Combustion experiments at different temperature steps revealed that the small amounts of isotopically light reduced carbon in BIFs, is recent organic contamination. From these results it is suggested that previous observations of low $\delta 13 \mathrm{C}$ values in Isua BIFs do not represent ancient remnants of life. An exception to our observations is a locality in the western part of the ISB, where isotopically light graphite occurs in a turbidite. Formation of graphite by siderite dissociation in this rock can be excluded, and thus this graphite could represent a remnant of life with a verified age of $3.8 \mathrm{Ga}$.

\section{The selective formation of RNA oligomers by montmorillonite catalysis}

Kong-Jiang Wang $\bullet N Y$ Center for Studies on The Origins of Life and Department of Chemistry, RPI, James P. Ferris $\bullet N Y$ Center for

Studies on the Origins of Life and Department of Chemistry

The clay-catalyzed RNA formation is the focus of this group. In this research, both adenosine and $\mathrm{CH}_{3} \mathrm{pA}$ were used as the initiators to simulate the elongation of the montmorillonite-catalyzed RNA oligomers.

In adenosine $+\operatorname{ImpA}$ and $\mathrm{CH}_{3} \mathrm{pA}+\mathrm{ImpA}$ reactions, 3', 5'-linked oligomers were preferentially elongated in montmorillonite-catalyzed reactions. The elongation efficiency of $3^{\prime}, 5^{\prime}$-linked dimers were more than 10 times higher than that of the corresponding $2^{\prime}-5^{\prime}$ linked dimers. The yield of the oligomers with two and more consecutive $2^{\prime}-5^{\prime}$ linkages at $3^{\prime}$-terminal was low. In adenosine $+\operatorname{ImpU}$ and $\mathrm{CH}_{3} \mathrm{pA}+\operatorname{ImpU}$ reactions, the $3^{\prime}, 5^{\prime}$-linked dimers were the much better initiators than the corresponding 2', 5'-linked dimers. Most or all of the oligomers longer than 5-mers were initiated by $\mathrm{A}_{3} \mathrm{pU}$ or $\mathrm{CH}_{3} \mathrm{pA}_{3} \mathrm{pA}$. The oligomers with one $2^{\prime}, 5^{\prime}$-linkage at $3^{\prime}$-terminal were found to be elongated by ImpU. However, the yield of oligomers with three and more consecutive $2^{\prime}, 5^{\prime}$-linkages at $3^{\prime}$-terminal were low. 
In the separate elongation experiments of both $7.5 \mathrm{mM} \mathrm{CH}_{3} \mathrm{pA}_{3} \mathrm{pA}$ and $7.5 \mathrm{mM} \mathrm{CH}_{3} \mathrm{pA}_{2} \mathrm{pA}$ by $7.5 \mathrm{mM}$ ImpA, the yield of $\mathrm{CH}_{3} \mathrm{pA}_{3} \mathrm{pA}-$ initiated trimers was much higher than that of $\mathrm{CH}_{3} \mathrm{pA}_{2} \mathrm{pA}$-initiated trimers. In the competitive elongation experiment $(3.75 \mathrm{mM}$ $\mathrm{CH}_{3} \mathrm{pA}_{3} \mathrm{pA}+3.75 \mathrm{mM} \quad \mathrm{CH}_{3} \mathrm{pA}_{2} \mathrm{pA}+7.5 \mathrm{mM}$ ImpA), the yield of $\mathrm{CH}_{3} \mathrm{pA}_{3} \mathrm{pA}$-initiated trimers was about 6 times higher than that of $\mathrm{CH}_{3} \mathrm{pA}_{2} \mathrm{pA}$-initiated trimers.

\section{Chemical constraints governing the origin of metabolism: the thermodynamic landscape of carbon group transformations \\ Arthur Weber $\bullet$ SETI Institute}

The free energy landscape of aqueous organic chemistry was investigated to identify the thermodynamic constraints that governed the chemistry of the origin of metabolism. Free energies were estimated for four types of reactions of biochemical importance - carbon-carbon bond cleavage and synthesis, hydrogen transfer between carbon groups, dehydration of alcohol groups, and aldo-keto isomerization. The energies were calculated for all possible aliphatic groups composed of carbon, hydrogen, and oxygen. Analysis of these organic transformation energies showed that the chemistry of the origin of metabolism (and to a lesser degree modern metabolism) is strongly constrained by: (1) the limited disproportionation energy of organic substrates that can be dissipated in a few irreversible reactions; (2) the energy dominance of a few halfreaction couples that determine whether carbon-carbon bond transformation (cleavage or synthesis) is irreversible, reversible, or prohibited; and (3) the dependence of the carbon group transformation energy on the type of participating functional groups (or oxidation level of involved groups) that in turn is contingent on prior reactions and precursors in the synthetic pathway.

From prebiotic molecules to early life and its context on Earth and elsewhere in the Universe

Frances Westall $\bullet C B M$, Bernard Barbier $\bullet C B M$, Maryléne Bertrand - $C B M$, Annie Chabin $\bullet C B M$, Brack André $\bullet C B M$

The Exobiology group at the Centre de Biophysique Moléculaire is engaged in experimental and documentational studies addressing the following problems.

(1) The origin of amino acids in space (photo-production of amino acids was achieved in simulated interstellar pre-cometary conditions) and in submarine hydrothermal vents (studies of black smoker materials).

(2) The influence of circularly polarised light on the emergence of homochirality using a synchrotron source (Orsay, France) to demonstrate asymmetric degradation of a racemic D,L-leucine mixture in the solid state.

(3) The influence of different minerals (crystalline CdS, metallic molybdenum and montmorillonite clay) on the polymerisation of amino acids into structured peptides.

(4) The investigation of simple, structured peptides containing Leu and Lys acting as enzymes in the catalysis of RNA degradation.
(5) The geological history of the early Earth as the context in which life arose and as a reference for the search for life on other planets, e.g. Mars.

(6) The documentation of the earliest life on Earth through investigations of fossil bacteria and their morphological and biochemical signatures.

(7) The search for life on Mars using early Earth as a reference and involvement in the evaluation of the Beagle 2 lander data.

Rotational spectroscopy and hot core observational searches for aminoethanol

Susanna Widicus $\bullet$ California Institute of Technology, Brian Drouin $\bullet$ California Institute of Technology; Jet Propulsion Laboratory, Kathryn Dyl $\bullet$ California Institute of Technology, Geoffrey Blake $\bullet$ California

Institute of Technology

Aminomethanol $\left(\mathrm{NH}_{2} \mathrm{CH}_{2} \mathrm{OH}\right)$ and aminoethanol $\left(\mathrm{NH}_{2} \mathrm{CH}_{2} \mathrm{CH}_{2} \mathrm{OH}\right)$ are predicted grain surface reaction products. These aminoalcohols are suspected to undergo hot core gas phase reactions to form the amino acids glycine and alanine, respectively. ${ }^{1}$ Observational searches for these molecules would offer insight into prebiotic mechanisms in hot cores. However, the necessary laboratory data to support such searches is incomplete.

Our characterization of aminoethanol in the $75-120 \mathrm{GHz}$ region and previous work in the $8-40 \mathrm{GHz}$ region ${ }^{2}$ has provided sufficient spectroscopic data for observational studies. Spectroscopic parameters, including standard rotational constants and fourth- and sixth-order centrifugal distortion constants, were determined for aminoethanol.

The hot core sources Orion KL and W51 have been surveyed for aminoethanol with the Owen's Valley Radio Observatory Millimeter Array. There was no detection of aminoethanol in these sources, and column density upper limits have been calculated. Higher-frequency laboratory work is planned and will support future searches in these and other hot cores.

References

${ }^{1}$ Charnley, S. (1999). Interstellar organic chemistry. In The Proceedings of the Workshop. The Bridge Between the Big Bang and Biology (Consiglio Nazionale delle Ricerche, Italy).

${ }^{2}$ Penn, R.E. \& Curl, R.F. (1971). J. Chem. Phys. 53, 651-658.

\section{Determination of pathways to glycine and alanine in} UV-irradiated ices containing water, methanol, and hydrogen cyanide via $a b$ initio quantum chemistry

\section{David Woon • Molecular Research Institute}

$A b$ initio quantum chemical methods were used to evaluate plausible pathways to the amino acids glycine (Gly) and alanine (Ala) in ices containing water, methanol, and hydrogen cyanide that are subjected to ultraviolet-irradiation. The calculations indicate that the acid group $\mathrm{COOH}$ is formed by the reaction between $\mathrm{OH}$ and $\mathrm{CO}$, the respective photolysis products of water and methanol. Successive hydrogenation of $\mathrm{HCN}$ yields $\mathrm{H}_{2} \mathrm{CN}, \mathrm{CH}_{2} \mathrm{NH}$ (methyleneimine), $\mathrm{CH}_{3} \mathrm{NH}, \mathrm{CH}_{2} \mathrm{NH}_{2}$, and ultimately $\mathrm{CH}_{3} \mathrm{NH}_{2}$ (methylamine). If $\mathrm{CH}_{2} \mathrm{NH}_{2}$ reacts with $\mathrm{COOH}$, Gly is formed. If it reacts with $\mathrm{CH}_{3}$ first and then with $\mathrm{COOH}$, Ala is formed.

\section{Mars}

\section{Mars Atmospheric Chemistry and Astrobiology Workshop summary}

Mark Allen • Jet Propulsion Laboratory; California Institute of Technology, Paul Wennberg • California Institute of Technology The Mars Atmospheric Chemistry and Astrobiology Workshop was held on the California Institute of Technology campus December 17-18, 2001. The prime objective of the workshop was to consider whether extant life beneath the surface, if it exists, would be in contact with the atmosphere and introduce a detectable signature in the atmosphere. To answer this question, the workshop also explored how well we understood the abiotic chemistry of the current atmosphere and other drivers of atmospheric composition (volcanoes, surface-atmosphere interactions, escape). The conclusions from this workshop will be presented.

\section{Revised geological understanding of Mars: implications for exobiological exploration} Victor Baker $\bullet$ University of Arizona, Shigenori Maruyama $\bullet$ Tokyo Institute of Technology, James Dohm $\bullet$ University of Arizona Recent discoveries reveal misleading aspects of the long-prevailing scientific view of an early warm-wet Mars progressively and irreversibly changing to cold-dry. First is the discovery of extensive water-related features (glacial, lacustrine, fluvial, periglacial, mass-movement) that 
indicate aqueous activity, probably operating episodically and during the most recent geological epochs on Mars. Second, and perhaps even more important, are the discoveries of extensive remnant magnetism in the rocks of the ancient highlands, evidence for extensive layering of the upper Martian crust, and various other anomalies, including ancient hematite deposits. These lead to a new understanding of Mars, unified under a theory that postulates an early phase of plate tectonics, associated with a primordial ocean and the rapid initiation and termination of a core dynamo, all occurring during the first several hundred million years of Martian history. This new theory of early Mars geological evolution is completely parallel to the earliest geological evolution of Earth, with the implication that studies of early Earth paleobiology are directly relevant to the exobiological exploration strategy for Mars.

\section{Mars remote sensing analog studies in Badwater} Basin, Death Valley, CA: groundtruth and analysis of surface samples

Alice Baldridge $\bullet$ Arizona State University, Jack Farmer $\bullet$ Arizona State University, Jeffrey Moersch • University of Tennessee

The detection of evaporites on Mars will have important implications for the role that liquid water has played in the planet's history (Farmer and Des Marais 1999). Orbital investigations of surface mineralogy are crucial to this exploration effort. Moersch (2000) previously conducted a spectral study of the Badwater Basin, Death Valley, CA to understand the limitations of the Thermal Emission Spectrometer (TES) and the Thermal Emission Imaging System (THEMIS) in detecting evaporite minerals. MODIS/ASTER Airborne Simulator (MASTER) data were degraded to TES and THEMIS resolution to examine the thresholds for detecting carbonates and sulfates. In this study we performed groundtruth of surface mineralogy to assess the capabilities of this spectrally based approach. The analysis utilized thin-section petrography, X-ray diffraction, electron microprobe and laboratory thermal emission spectrometer analysis.

Interpretations made by Moersch agree with our analysis for $70 \%$ of the surface samples. The major mineral component abundance was measured from thin-section point counting to be at least $20 \%$ for sulfates and $15 \%$ for carbonates. Evaporite basin mineralogy can be quite complex and the spectral data can be confounded by a variety of factors including natural mixtures of salts with silicates. This complexity is being addressed to lay the groundwork for accurate identification of these minerals on Mars.

\section{References}

Farmer, J.D. \& Des Marais, D.J. (1999). Exploring for a record of ancient Martian life. Journal of Geophysical Research-Planets 104(E11), 26 977-26995.

Moersch, J.E. (2000). Detectability of Martian evaporites - terrestrial analog studies with MASTER data. 31st Lunar and Planetary Science Conference, Houston, TX.

\section{A mineralogical instrument for Mars exobiology studies \\ David Blake $\bullet$ NASA Ames Research Center, Philippe Sarrazin $\bullet$} NASA Ames Research Center, David Bish $\bullet$ Los Alamos National Laboratory, David Vaniman $\bullet$ Los Alamos National Laboratory, Steve Chipera $\bullet$ Los Alamos National Laboratory, Stewart Collins $\bullet$ Jet Propulsion Laboratory

Carbonate minerals in the ancient Martian meteorite ALH84001 are the only solids that bear witness to the processing of volatile and biologically critical compounds $\left(\mathrm{CO}_{2}, \mathrm{H}_{2} \mathrm{O}\right)$ on early Mars. Nearly identical carbonate globules have been found in xenoliths and their host basalts from Quaternary volcanic centers in northern Spitsbergen (Norway). Our data suggest that these globules were deposited in situ by hydrothermal (e.g. hot spring) waters associated with volcanic activity. By analogy with the Spitsbergen carbonates, it seems likely that the ALH84001 carbonates were deposited in a hydrothermal environment as well. Hydrothermal activity was probably common and widespread on early Mars, which featured abundant basaltic rocks, water as ice or liquid, and heat from volcanoes or asteroid impacts. On Earth, descendents of the earliest known forms of life prefer hydrothermal environments, and it remains possible that similar settings on Mars could have hosted early forms of life.

\section{Hydrothermal origin for carbonate globules In Martian meteorite Alh8400I : a terrestrial analog from Spitsbergen (Norway)}

David Blake $\bullet$ NASA Ames Research Center, Allan Treiman $\bullet$ Lunar and Planetary Institute, Subarnarekha De $\bullet$ NASA Ames Research Center, Hans Amundsen $\bullet$ University of Oslo, Ted Bunch $\bullet$ Northern Arizona University

Carbonate minerals in the ancient Martian meteorite ALH84001 are the only solids that bear witness to the processing of volatile and biologically critical compounds $\left(\mathrm{CO}_{2}, \mathrm{H}_{2} \mathrm{O}\right)$ on early Mars. Nearly identical carbonate globules have been found in xenoliths and their host basalts from Quaternary volcanic centers in northern Spitsbergen (Norway). These globules were deposited in situ by hot (i.e. hydrothermal) waters associated with the volcanic activity. Like the Spitsbergen carbonates, the ALH84001 carbonates were probably deposited by hot water. Hydrothermal activity was probably common and widespread on early Mars, which featured abundant basaltic rocks, water as ice or liquid, and heat from volcanoes or asteroid impacts. An important component of early life on Earth preferred hydrothermal environments, which are now shown to have been present on early Mars.

\section{Astrobiological implications of modern glaciers and surface ice on Mars}

Nathalie A. Cabrol $\bullet$ SETI; NASA Ames Research Center, Edmond A. Grin • SETI; NASA Ames Research Center

A global survey of the MOC images shows that gullies are far from being the only evidence supporting a significant climate change in recent Mars history. We have catalogued nearly 500 pristine landforms whose characteristics are similar to terrestrial mudflows, striped valleys, rock glaciers, and debris-covered glaciers. They are localized in a strict latitude and altitude domain and show a clear morphological and environmental continuum best explained by a recent climate change that could have involved a global warming with snow precipitation and/or a massive South Pole advance to the mid-latitudes. Their presence requires the accumulation of large volumes of ice recently at the surface of Mars. Our hypothesis is based on geological observations and provides clues for climate modelers (e.g. altitudes, latitudes, slopes) to reconstruct the conditions necessary to generate the environment required by these landforms. Moreover, these massflows occur within the regions where the Mars Odyssey mission is recording high-hydrogen signals, indicating possibly that a non-negligible volume of ice is still present near the surface protected by a mantle of debris. These modern massflows have a high astrobiological potential. They could still contain a significant proportion of ice in their core that constitutes a possible resource for future missions and for life. Geological materials pertaining to ancient periods were brought to the surface recently by these creep and flow processes. They may contain fossils and/or traces of extant micro-organic communities normally sheltered in the subsurface and now within grasp.

The nature of coarse-grained crystalline hematite and its implications for the early geochemical and biological environment on Mars

David Catling $\bullet$ University of Washington, Jeff Moore $\bullet$ NASA Ames Research Center

The Thermal Emission Spectrometer (TES) on the Mars Global Surveyor spacecraft has detected deposits of coarse-grained, gray crystalline hematite in Sinus Meridiani, Aram Chaos, and Vallis Marineris. This hematite appears to be specular (i.e. it is specularite). We discuss physicochemical mechanisms for forming specularite. A key to the origin of the specularite is that it requires (re-)crystallization at temperatures in excess of $\sim 100{ }^{\circ} \mathrm{C}$. Thermal processing could occur (1) in diagenesis at a depth of a few $\mathrm{km}$ of sediments originally formed in low-temperature waters, or (2) in slow precipitation from hydrothermal 
solution. The topography in Aram Chaos suggests that hematite was formed in planar beds and has since been exposed by erosion, with some detrital hematite swept to lower elevations. The topography and chaotic terrain may favor a hydrothermal aquifer as the original setting where hematite formed. Redox balance also implies that much hydrogen must have been evolved to complement the extensive oxidation. Rate-limited escape of hydrogen to space would have led to a significant hydrogen mixing ratio in the early atmosphere $\sim 10^{-3}$. The coexistence of several factors implied by the hematite deposits would have been favorable for primitive organisms on early Mars. These factors include liquid water, and abundant electron donors $\left(\mathrm{H}_{2}\right)$ and acceptors $\left(\mathrm{Fe}^{3+}\right)$.

\section{Magnetite crystal chains in the Martian meteorite ALH8400I and in terrestrial magnetofossils}

E. Imre Friedmann $\bullet$ NASA Ames Research Center, Jacek Wierzchos $\bullet$ Universitat de Lleida, Spain, Carmen Ascaso $\bullet$ Centro de Ciencias Medioambientales, CSIC, Madrid, Spain

In magnetotactic bacteria, magnetite crystals are arranged in linear chains, yet such chains have not been demonstrated to date in fossil materials preserved in hard sediments. The reason is that preparation for transmission electron microscopy requires acid extraction of the crystals, which destroys the chains. For our study of magnetite crystal chains in the meteorite ALH84001, we developed a method (highmagnification backscattered scanning electron microscopy) that permits visualization of crystal chains in situ within the intact rock (PNAS 98, 2176-2181, 2001).

This method reveals the presence of numerous magnetite crystal chains in a 300000 year old terrestrial magnetofossil specimen from Mururoa atoll, described by Aissaoui et al. (Earth Planet. Sci. Lett. 97 , 102-112, 1990). The images from Martian and from terrestrial material are highly similar.

Our results disprove the contention of Buseck et al. (PNAS 98, 13490-13495) that the apparent absence of magnetite chains in the paleontological record is a sign that "such chains are unlikely to survive geological processes." We can also show that all other criticisms in the Buseck et al. paper of our report of magnetite crystal chains in ALH84001 are based on error, on lack of familiarity with paleontological processes, or on misunderstanding of the physical basis of the electron-microscopic method we used.

\section{Evolution of Mars: a model to support life as} reflected by Martian meteorites and spaceflight data Everett Gibson $\bullet$ NASA Johnson Space Center, Frances Westall • CNRS, Orleans, France, David S. McKay $\bullet$ NASA Johnson Space Center, Kathie Thomas-Kerpta $\bullet$ Lockheed Martin; NASA Johnson Space Center, Susan J. Wentworth $\bullet$ Lockheed Martin; NASA Johnson Space Center, Simon J. Clemett Clemett $\bullet$ Lockheed Martin; NASA Johnson Space Center

Martian meteorites have crystallization ages from 165 to $4500 \mathrm{Ma}$. They record the geological history of Mars from the Noachian to the Hesperion and Amazonian. Samples of the early Martian crust (ALH84001) show a period of intensive bombardment and reflect the magnetic history of the planet along with a record of low-temperature depositions. Carbonate globules reflect evaporation processes at $3900 \mathrm{Ma}$. Evidence for magnetotactic bacteria in the carbonates is shown by the unique magnetite populations. Oxygen isotopic compositions of silicates and volatiles show two distinct oxygen reservoirs on Mars. Significant environmental changes took place after $3800 \mathrm{Ma}$ during the early Hesperion. Mars lost its atmosphere, cooled down, and surficial waters disappeared, probably forming a cryosphere. Loss of near-surface waters resulted in evaporites in low-lying regions. If life ever developed on Mars, it would have disappeared from the surface to take refuge in the more water-rich subsurface. Periodic volcanism continued during the Amazonian, as shown by the nakhlite and shergottite ages (1300-165 Ma). Evidence of aqueous alteration within these meteorites is reflected by secondary mineralization. Associated features have fossilized bacteriomorph characteristics. If these features are indigenous and biogenic, this would imply life in the Martian groundwater system. Within the framework of this history, the SNC meteorites and their secondary mineral deposits plus bacteriomorphs and organic signatures could contain a possible record of living organisms on Mars.

\section{Carbon isotopic characteristics of the Martian magmatic minerals: insights into Martian carbon reservoirs}

Julia S. Goreva $\bullet$ Arizona State University, Yunbin Guan $\bullet$ Arizona State University, Laurie A. Leshin • Arizona State University

The isotopic characteristics of carbon reservoirs on Mars are of interest because of their implications for the carbon inventory of the planet, and the delimitation of potential biological signatures. Ironically, among atmosphere, crust and mantle, the largest reservoir (interior) is the least defined. Magmatic phosphates are phases whose structure incorporates significant amounts of $\mathrm{C}$, thus preserving juvenile $\mathrm{C}$ isotopic signatures. We have analyzed $\mathrm{C}$ isotopes of phosphates in the basaltic shergottite Los Angeles. The average $\delta 13 \mathrm{C}$ of apatites is $-13.7 \pm 2.6 \%$ and of whitlokites is $-18.3 \pm 4.0 \%$ (1s). These values are higher than previously reported for Martian magmatic carbon $(<-22 \%)$ and significantly lower than terrestrial magmatic carbon $(-5 \pm 2 \%)$. This could reflect changing $\delta 13 \mathrm{C}$ through degassing/cooling, or it may be an indication that Mars has lighter mantle carbon than Earth. The former implies that the magmatic $\delta 13 \mathrm{C}$ of Mars at the time of phosphate crystallization was even higher than measured, approaching the terrestrial value. The latter is consistent with the idea of Mars accretion from volatile-rich material followed by little crustal recycling, thus preserving more primordial $\mathrm{C}$ isotopes. In either case, our results are critical for making estimates of the amount of $\mathrm{C}$ lost from the atmosphere to produce its high $\delta 13 \mathrm{C}$ value, and for placing potential biosignatures into an isotopic context.

\section{HiRISE: the people's camera for Mars Reconnaissance Orbiter}

Virginia Gulick $\bullet$ NASA Ames Research Center; SETI Institute, Alfred McEwen $\bullet$ University of Arizona, Alan Delamere $\bullet$ Ball Aerospace \& Technologies Corporation, Candy Hanson $\bullet$ Jet Propulsion Laboratory, Eric Eliason • U.S. Geological Survey, Flagstaff, HiRISE team $\bullet$ USA \& Europe

The High Resolution Imaging Science Experiment (HiRISE) will fly on the Mars Reconnaissance Orbiter (MRO) mission, planned for launch in August of 2005. HiRISE will investigate deposits and landforms resulting from geological and climatic processes and is optimized for the evaluation of candidate landing sites. The camera will combine unprecedented resolution $(25-50 \mathrm{~cm} / \mathrm{pixel})$ and signal-to-noise ratio (SNR $>100: 1$ at all latitudes) with a 5-10 km swath width, partial three-color coverage, and image $>2 \%$ of Mars at $1 \mathrm{~m} /$ pixel or better. Stereo image pairs will be acquired over the highest-priority locations allowing for a relative vertical precision of $<25 \mathrm{~cm}$. By combining very high resolution and SNR with a large swath width, it is possible to image on a variety of scales down to $1 \mathrm{~m}$, a scale currently afforded only in glimpses by landers. HiRISE will offer such views over any selected region of Mars, providing a bridge between orbital remote sensing and landed missions.

The HiRISE team welcomes and will facilitate involvement by the science community and the public. All team members are involved in HiRISE's E/PO effort. User-friendly web tools will be available to both the science community and the public to view/analyze HiRISE images and to submit observation requests. Processed images will be released soon after acquisition to allow everyone to share in the scientific discovery process.

\section{Discriminating between glasses and phyllosilicates in thermal infrared emission spectra: implications for the identification of water-related phases on Mars Victoria Hamilton • Arizona State University} Numerous Martian spectra from the MGS TES were initially interpreted as representing an andesitic surface type due to the presence of a glass component. Subsequent analyses have suggested that the glass com- 
ponent potentially has been confused with a sheet silicate component due to qualitative similarities in the band shapes of silica-rich glasses and phyllosilicates of the smectite group. Deconvolutions of Martian data rely on the distinctiveness of all geological phases' infrared band shapes, positions, and presence to obtain meaningful mineralogical results. Therefore, a thorough understanding of the ability to distinguish between glasses and certain phyllosilicates using the deconvolution technique (and the accuracy in doing so) is critical to our interpretations of the degree and type of alteration and/or weathering on the Martian surface (including the role of water). To test the discriminability of these phases by deconvolution, we have generated spectra representing mixtures of these minerals in varying proportions (as well as some including igneous phases) and deconvolved them using end member sets that both include and exclude the spectra used to make the mixed spectrum. In this way, we can quantitatively assess the uncertainties associated with distinguishing between glasses and certain phyllosilicates in thermal infrared spectra of multimineralic materials.

\section{Stability of salt springs on Mars}

Jennifer Heldmann $\bullet$ University of Colorado, Boulder, Brian Toon • University of Colorado, Boulder, Chris McKay $\bullet$ NASA Ames Research Center, Dale Anderson $\bullet$ McGill University, Wayne Pollard $\bullet$ McGill University

Geological evidence for recent liquid water outflows on Mars suggests that these events occurred under present climatic conditions with mean surface temperatures of $-60{ }^{\circ} \mathrm{C}$ and extensive permafrost. Such findings imply that liquid water must have been stable on the Martian surface for a long enough period of time to produce the observed outflow channels. Aqueous brine solutions would be stable against boiling on the surface of Mars due to the vapor pressure depression of the saline solution. However, the solution does not necessarily have to be above its freezing point as water will still flow before icing over. Preliminary modeling results regarding the expected lifetimes of liquid water flows are presented. We also examine cold perennial saline springs in the Canadian High Arctic that flow through thick continuous permafrost as an analog for such spring activity on Mars.

\section{Martian impact crater lakes: geophysical modeling} and connections to the hunt for life on Mars

Andrew N. Hock • University of California; IGPP Center for Astrobiology, William B. Moore $\bullet$ University of California; IGPP Center for Astrobiology, Nathalie A. Cabrol $\bullet$ SETI Institute; NASA Ames Research Center, Edmond A. Grin • SETI Institute; NASA Ames Research Center

Life as we understand it is dependent on a source of liquid water, energy, and nutrients. In cases where sunlight is not available, or the surface environment proves inhospitable - such as the situation for present-day Mars - thermal activity and hydrothermal systems can supply energy to subsurface environments (Newsom 1980). Sources of hydrothermal energy can originate from both recent volcanic activity (Hartmann et al. 2000) and large impact craters (Newsom 1980; Newsom et al. 1996; Cabrol \& Grin 1999, 2001). Though a discontinuous source of heat, impact crater lakes may have been one of the few environments on Mars where liquid water, energy, and a source of nutrients existed. Therefore, characterizing the lifetime of crater lakes (and associated hydrothermal circulation) is vital to understanding the constraints placed on any biological system that may have existed there. Here, we present a model that builds on previous work studying the contribution and lifetime of different heat sources (e.g. Onorato et al. 1978; Newsom et al. 1996; Daubar \& Kring 2001) that includes the effects of hydrothermal circulation and latent heat from impact melt solidification. A preliminary calculation (e.g. Turcotte \& Schubert 1982) of the solidification timescale for an impact melt sheet on Mars gave an estimate of 50000 years for a $100 \mathrm{~km}$ crater.

References

Cabrol, N.A. \& Grin, E.A. (1999). Distribution, classification, and ages of Martian impact crater lakes, Icarus 142, 160-172.

Cabrol, N.A. \& Grin, E.A. (2001). Evolution of lacustrine environments on Mars (is Mars only hydrologically dormant?). Icarus 149, 291-328.
Daubar, I.J. \& Kring, D.A. (2001). Impact-induced hydrothermal systems: heat sources and lifetimes, Lunar and Planet. Sci. Conf. XXXII, abstract 1727 .

Hartmann, W.K., Grier, J.A., Berman, D.C., Bottke, W., Gladman, B., Morbidelli, A., Petit, J.-M. \& Dones, L. (2000). Martian chronology: new Mars global surveyor results of absolute calibration, geologically young volcanism, and fluvial episodes. Lunar and Planet. Sci. Conf $X X X I$, abstract 1179 .

Newsom, H.E. (1980). Hydrothermal alteration of impact crater melt sheets with implications for Mars. Icarus 44, 207-216.

Newsom, H.E., Britell, G.E., Hibbitts, C.A., Crossey, L.J. \& Kudo, A.M. (1996). Impact crater lakes on Mars. J. Geophys. Res., 101, 14951-14955

Onorato, P.I.K., Uhlmann, D.R. \& Simmons, C.H. (1978). The thermal history of the Manicouagan impact melt sheet, Quebec. J. Geophys. Res. 83, 2789-2798.

Turcotte, D.L. \& Schubert, G. (1982). Geodynamics: Application of Continuum Physics to Geological Problems Wiley: New York.

\section{Was early Mars warmed by $\mathrm{CH}_{4}$ ?}

Hilary Justh • Pennsylvania State University, James Kasting • Pennsylvania State University

Planetary mission images have shown Martian geological features that seem to indicate an earlier period of hydrologic activity suggesting a climate with a $T_{\mathrm{s}}$ of $273 \mathrm{~K}$ or higher. The presence of liquid water would require a larger greenhouse effect than presently needed since $S_{0}$ was $25 \%$ lower 3.8 billion years ago. Research into the effects of $\mathrm{CO}_{2}$ clouds upon the early Martian climate yielded results that would not effectively warm the surface. Forget and Pierrehumbert (Science, 1997) showed that large $\mathrm{CO}_{2}$ ice crystals in upper tropospheric clouds generate a strong warming effect. Obtaining mean surface temperatures above $273 \mathrm{~K}$ requires $100 \%$ cloud cover, an unrealistic condition. Reduction in cloud cover makes achieving warm surface temperatures difficult except at high pressures. Low and optically thick $\mathrm{CO}_{2}$ clouds could cool the surface.

$\mathrm{CH}_{4}$ has been suggested as an important greenhouse gas on the early Earth, leading us to look at $\mathrm{CH}_{4}$ as a solution to the early Mars climate issue. To investigate the possible warming effect of $\mathrm{CH}_{4}$, we utilized a modified, one-dimensional, radiative-convective climate model. Calculations of the effects of $\mathrm{CH}_{4}$ upon the early Martian climate will be presented. The use of $\mathrm{CH}_{4}$ does not necessarily imply the presence of life. Abiotic sources of $\mathrm{CH}_{4}$, such as serpentinization, could supply the required concentrations.

\section{Spatial trends in crater modification and valley network formation across the Martian highlands during the Noachian period}

Marc Kramer $\bullet N A S A$ Ames Research Center, Chris Potter $\bullet$ NASA

Ames Research Center, David Des Marais $\bullet N A S A$ Ames Research

Center, David Peterson $\bullet$ NASA Ames Research Center

Proliferation of microbial life on an arid planet like ancient Mars most likely occurred with an active hydrologic cycle and perhaps the presence of liquid water on the surface. If large-scale atmospherics delivered water to the surface, regions of high precipitation probably experienced enhanced rates of erosion, deposition and consequently greater evidence of crater degradation, and valley network formation. Conversely, if subsurface water dynamics and ice/volcanic interactions are responsible for the fluvial-like features on Mars, this would mean more extreme and episodic environmental conditions for the persistence of life. In the latter hydrologic scenario, we would also expect trends in crater degradation and valley network formation to be more localized rather than regionally trending within climatic or precipitation zones. The ancient highlands contain the most complete record of geological processes and clues to past possible climates on Mars. Craters formed during the late heavy bombardment on Mars have been modified by a variety of geological processes including eolian, volcanic, and fluvial activity. We are evaluating spatial trends in the extent of crater modification and valley network formation across the ancient highlands 
to better understand the role that precipitation, subsurface water dynamics, and other geological processes may have played in surface modification.

\section{On the existence and stability of liquid water on the surface of Mars today}

Lawrence Kuznetz $\bullet$ NASA; NSBRI, David Gan $\bullet$ University of California, Berkeley

"Liquid water does not exist on the surface of Mars ... without liquid water, life as we know it cannot exist." Viking Principal investigator Norman Horowitz made these statements over two decades ago, establishing the contemporary paradigm of a barren Mars today. Since that time, a wealth of new knowledge has been accumulated on soil, air composition and climate from the Viking, Pathfinder and Mars Global Surveyor (MGS) robotic probes. We now have extensive pressure and temperature data demonstrating pressures above the triple point and temperatures above freezing for long periods of time, meeting the criteria for liquid water. To shed more light on this issue, a multi-tiered study of theoretical analysis and ground-based experiments has been initiated. This article presents the first results from that study.

\section{Mineralogical mapping of Mars using data-derived endmembers}

Sara E. Martinez-Alonso $\bullet$ University of Colorado, Boulder

Life on early Earth seems to have appeared in environments such as hydrothermal systems, where liquid water, biogenic elements, and a source of energy were available. Hydrothermal minerals can be identified through their infrared spectra by instruments such as MGS-TES.

Most of our present understanding of the surface mineralogy of Mars derives from analysis of TES data based on unconstrained linear deconvolution techniques and finite libraries of reference spectral endmembers measured in the laboratory (Christensen et al. 2001).

In this study we use a different approach, based on deriving the reference spectral endmembers from the TES emissivity data themselves. In order to identify the most "spectrally interesting" areas of Mars, a global spectral dimensionality index is being calculated for $5 \times 5-\mathrm{deg}^{2}$ zones. This is accomplished by applying to the data a minimum noise fraction transformation (Green et al. 1988; Boardman and Kruse 1994) to determine its dimensionality, segregate the noise, and reduce the computational requirements for subsequent processing. Zones of higher spectral dimensionality are then further analyzed. The spectrally purest spectra are extracted using the pixel purity index technique (Boardman et al. 1995) and visually identified. Finally, quantitative mapping of endmembers at the sub-pixel level is achieved using constrained linear unmixing techniques.

\section{Layered deposits on Mars: clues to past climate history}

Sabrina Mayerberger $\bullet$ University of California, Los Angeles; IGPP, Bruce Runnegar • University of California, Los Angeles; IGPP Determining the past climate history of Mars is essential in order to understand the planetary evolution of the red planet and possible habitats for life. Layered deposits at the Martian polar caps contain a wealth of information regarding the recent history of the planet and its volatiles. Mars Global Surveyor's Orbiter Camera (MOC) monitors daily Martian weather, dust storms, and polar cap changes. Recently released images address a number of issues relating to the physical properties of the Martian polar residual caps and associated layered deposits. Detailed quantitative analysis of those layered deposits should give a better understanding of the properties and climate history of the Martian polar regions and in turn, contribute to the determination of future landing sites.

\section{Habitability of near-surface environments on Mars Abel Mendez $\bullet$ UPR, Arecibo}

Ice-rich near-surface environments on Mars may provide a protective layer and a temporary habitat for microbial life. This work studies with theoretical and experimental work the habitability of the Martian surface in more detail than previous research. First, the Martian nearsurface microenvironment is modeled to describe possible habitats at microbial relevant space and time scales. Second, the theory of environmental biophysics is used to quantify rates of mass and energy transfer between potential microorganisms and the Martian environment. Third, an extensive biodiversity database of physical, chemical and biological requirements of prokaryotes is used to suggest possible interactions of microorganisms with the Martian environment. Finally, experimental work is being done to measure microbial growth under simulated daily Martian conditions. The data suggests that some nearsurface equatorial regions on Mars may have the physical environment to support microbial growth provided the other requirements of life are present. Growth will be limited to a few hours during the day at very low growth rates. This suggests that the potential to contaminate the planet from Earth's biota is larger than previously thought. Also, growth of indigenous microbial forms under the present Mars environment is an open possibility.

\section{Degradation and detection of biological matter using low-temperature plasmas}

R. Mogul $\bullet$ NASA Ames Research Center; SETI, A. Bol'shakov • NASA Ames Research Center; SETI, S.L. Chan $\bullet$ NASA Ames Research Center; SETI, H. Imanaka $\bullet N A S A$ Ames Research Center; SETI, B. Khare $\bullet$ NASA Ames Research Center; SETI, M.

Meyyappan $\bullet$ NASA Ames Research Center; SETI, J. Trent $\bullet$ NASA Ames Research Center; SETI

The detection of extant life is of paramount importance to any upcoming mission to Mars. Life is dependent on both the presence and function of biological molecules such as proteins, lipids, carbohydrates and DNA. Thus, detection of any one of these macromolecules would be an indicator for complex bioorganic chemistries. In order to develop an effective sterilization technique for life-detecting space probes, we have investigated the use of low-temperature plasmas. In addition, we are exploring the use of "cold plasmas" as a means of generating biosignatures from solid samples such as soil and rock. This research is based on the volatilization chemistry of low-temperature plasmas and would have application in: (a) planetary protection, (b) the development of plasma-based life detection methods and (c) characterization of the degradation of biomatter in an ionizing Martian atmosphere. We have been performing several microbiological, biochemical and spectrophotometric experiments using a set of model biological samples (a microbe, polypeptide and nucleic acid) and plasmas generated from different gases (oxygen and a Martian atmospheric analog). In all, we suggest that cold plasmas are an important new tool in astrobiology.

Terrestrial analogs for Mars evaporite environments: Storr's Lake, Bahamas, and the Dead Sea, Israel as two case studies

Penny Morris • University of Houston Downtown, Susan Wentworth $\bullet$ Lockheed Martin, Monica Byrne $\bullet$ Wellesley College, Robin Brigmon • Savannah River Company, Carlton Allen $\bullet$ NASA Johnson Space

Center, David McKay • NASA Johnson Space Center

Terrestrial evaporites can be marine or non-marine. Depending on the source waters, their chemistry will vary. The chemistry will affect the biota and the fossilization processes. Terrestrial evaporite biota can help us understand microbial fossilization processes in extreme environments, and will provide a baseline for interpreting some Mars meteorites and Mars sample return rocks.

Storr's Lake benthic biotas are incorporated into stromatolitic structures composed of abundant biofilm, rods, filaments (fungal hyphae, filamentous bacteria), cocci (abiotic and biotic), and diatoms. The primary fossilized mineral(s) are calcium carbonate enriched with magnesium. Ovoid- to rod-shaped forms may represent sulfate-reducing bacteria identified by R. Brigmon by $16 \mathrm{~S}$ rRNA (personal communication).

The Dead Sea benthic biota is less diverse. Our samples contain filaments (cyanobacteria and possibly fungi), rod-shaped to ovoidshaped bacteria, some biofilm deposits, and Coccolithus pelagica (coccolith). Calcium carbonate is the primary fossilization mineral. 
Both Storr's Lake and the Dead Sea represent important terrestrial analogs and rich resources for understanding the adaptations required for organisms to survive in evaporites. If life occurred early in Mars history, survival depended on genetic selection that allowed some groups to live and reproduce in high salinity environments.

\section{Carbon isotopic composition of Alh8400 I carbonates: an ion microprobe study}

Paul Niles • Arizona State University, Laurie Leshin • Arizona State University, Yunbin Guan • Arizona State University, Rick Hervig • Arizona State University

Carbon isotopic compositions of ALH84001 carbonates have been measured on a microscale using the IMS $6 \mathrm{f}$ ion microprobe at Arizona State University. ALH84001 carbonates show a large variation in carbon isotopic values from $27.6 \pm 2.1$ to $50.6 \pm 2.9$ per mil relative to PDB. The data show a general correlation between carbon isotopic composition and mole fraction of magnesium, with the most magnesium carbonates having the heaviest carbon isotopic compositions. These results firmly establish carbon isotopic variability in ALH84001 carbonates and considerably expand the range of values obtained by conventional techniques. ${ }^{1,2}$

The data sample a restricted portion of the total range of carbonate compositions, but the closed system hypothesis proposed by Leshin et $a l .^{3}$ can be ruled out as a possibility for the formation of the ALH carbonates. It seems likely that another isotope fractionating process was involved during the formation of the ALH84001 carbonates. This process might include extensive $\mathrm{CO}_{2}$ degassing caused by evaporation, eruptive decompression, or freezing.

References

1 Jull, A.J.T. et al. (1998). Science 279, 366-369.

2 Romanek, C.S. et al. (1994). Nature 372, 655-657.

${ }^{3}$ Leshin, L.A. et al. (1998). Geochimica Et Cosmochimica Acta 62, 3-13.

\section{Oxidant transport in the Martian regolith}

Richard Quinn • SETI Institute, Aaron Zent $\bullet$ NASA Ames Research Center

One of the more surprising results of the Viking missions was the discovery of a chemically reactive regolith that was free of organics, even in subsurface samples shielded from ultraviolet light. Explaining these results entirely by photochemically produced surface radicals requires that these species undergo surface diffusion across distances of at least $10 \mathrm{~cm}$. Alternatively, the surface mobility of the radicals could be very low, and they could enjoy extraordinary chemical stability. This would permit physical mixing of the regolith to move the oxidized material. Another possible mechanism for oxidant transport is vapor diffusion of photochemically produced species. In this case, diffusion is limited by the rate of heterogeneous catalytic destruction of these species by the Martian surface material. Our previous work, using palagonite as a regolith analog, indicated that peroxide diffusion would be limited to a few centimeters. Although palagonite is a good spectral analog, it is likely that the hydroxylation state of most palagonites is much too high to be an appropriate analog for Martian surface chemistry simulations. New experiments using iron-rich analogs with carefully controlled hydroxylation states indicate that the rate of catalytic destruction of hydrogen peroxide in the Martian regolith may be lower than previously thought.

\section{Martian soil, solar UV radiation and spores: the experiment MARSTOX}

Petra Rettberg $\bullet$ DLR, Institute of Aerospace Medicine, Koeln, Germany, Elke Rabbow $\bullet$ RWTH Aachen, Institut for Flugmedizin, Aachen, Germany, Corinna Panitz $\bullet$ DLR, Institute of Aerospace Medicine, Koeln, Germany, Gerda Horneck $\bullet$ DLR, Institute of Aerospace Medicine, Koeln, Germany, Richard Waesch $\bullet$ DLR, Institute of Space Sensor Technology and Planetary Exploration Berlin, Germany, Heinrich Waenke $\bullet$ Max Planck Institute for Chemistry, Mainz, Germany

The early histories of Mars and Earth show some similarities during the period when life emerged on Earth. The experiment MARSTOX of the ESA FOTON M-1 mission, scheduled for the end of 2002, will be a preparatory step for the future exploration of Mars as another planet which has had the potential for the evolution of life. The interference of Martian soil components and the intense and nearly unfiltered Martian solar ultraviolet (UV) radiation with terrestrial resistant microbial forms, bacterial spores of Bacillus subtilis, will be tested. Different types of Mars soil analogs will be used to determine, on one hand, their potential toxicity alone or in combination with solar UV (phototoxicity) and, on the other hand, their UV protection capability. Two sets of samples will be used under different cut-off filters used to simulate the UV radiation climate of Mars and Earth. After exposure in space in the ESA hardware BIOPAN, the survival of and mutation induction in the spores will be analyzed at DLR together with parallel samples from the corresponding ground control experiment performed at DLR. This experiment will provide new insights into the adaptation to environmental extremes on Earth or other planets that define the principal limits of life and at the same time bear the potential for the evolution and distribution of life.

\section{Survival of Bacillus subtilis on spacecraft materials under simulated Martian environments}

Andrew C. Schuerger $\bullet$ Dynamac Corporation, Rocco L. Mancinelli $\bullet$ SETI Institute, NASA, Ames Research Center, Roger G. Kern $\bullet$ Jet Propulsion Lab., Calif. Institute of Technology, Lynn J. Rothschild • Ecosystem Science and Technology Branch, NASA, Ames Research Center, Christopher P. McKay $\bullet$ Space Science Division, NASA, Ames Research Center

Critical to the success of near-term Mars lander missions in which life detection and/or organic-detection payloads are flown will be modeling the microbial ecology of outbound spacecraft, and predicting whether terrestrial microorganisms might survive, grow, and replicate on Mars. If terrestrial microorganisms present on spacecraft surfaces survive on Mars, then their presence might affect the success of these experiments. Furthermore, the effects of Martian environments on the survival of terrestrial microorganisms will contribute to the selection of appropriate sanitation and sterilization protocols used during spacecraft assembly. Several studies in the literature indicate that microorganisms can survive in extraterrestrial environments if they are shielded from ultraviolet (UV) irradiation from the Sun. However, no studies have appeared in the literature that simulate the survival of terrestrial microorganisms on spacecraft components under realistic conditions of temperature, gas composition, pressure, and UV irradiation expected on Mars.

Experiments were conducted in the Mars Simulation Chamber (MSC) located in the Operation and Checkout building at Kennedy Space Center, FL. The MSC is a moderately sized low-pressure chamber that has an internal volume of approximately $2 \mathrm{~m}^{3}$. The MSC was used to create Mars surface environments in which pressure $(8.5 \mathrm{mb})$, temperature $\left(-80\right.$ to $+23{ }^{\circ} \mathrm{C}$ ), gas composition (Earth-normal $\mathrm{N}_{2} / \mathrm{O}_{2}$ mix, pure $\mathrm{N}_{2}$, pure $\mathrm{CO}_{2}$, or a Mars gas mix), and UV-VIS-NIR fluence rates $(200-1200 \mathrm{~nm})$ were maintained within tight limits. The temperature was maintained at specific set points $\left(-80,-40,-10\right.$, or $\left.+23^{\circ} \mathrm{C}\right)$ by a liquid-nitrogen cold plate mounted within the MSC. The Mars gas mix was composed of $\mathrm{CO}_{2}(95.3 \%), \mathrm{N}_{2}(2.7 \%), \mathrm{Ar}(1.6 \%), \mathrm{O}_{2}(0.13 \%)$, and water vapor $(0.03 \%)$. Experiments were conducted to measure the effects of pressure, gas composition, and temperature alone or in combination with a normal Mars UV-VIS-NIR light environment. The Mars UV-VIS-NIR light environment was created by using two $450 \mathrm{~W}$ xenon arc lamps mounted on the outside of the MSC. The UV-VISNIR light was brought into the MSC through two bulkhead fittings configured with UV-transmitting fiber-optic bundles, and the light was distributed within the MSC using a set of eight fiber-optic bundles. The UV light distribution system permitted UV-VIS-NIR light to be precisely directed to various spacecraft components mounted in specially designed microbial sampling device (MSD) holders. Different grades of neutral density filters were placed in the aluminum tops of the MSD units to simulate first-order approximations of UV attenuation by Martian atmospheric dust. The neutral density filters simulated optical depths of $\tau=0.1,0.5,0.7,1.4,2.5$, or 3.5. Dormant endospores of the bacterium Bacillus subtilis were deposited on various spacecraft materials in such a way as to create $1-\mathrm{cm}$ diameter monolayers of viable 
bacteria at a mean spore density of $2.47 \times 10^{6}$ per sample. The aluminum coupons containing the bacterial monolayers were placed within the MSD units, the MSD units were mounted on the liquid-nitrogen cold plate within the MSC, and the bacterial populations subjected to various experimental conditions.

Populations of $B$. subtilis placed on aluminum coupons and subjected to an Earth-normal temperature $\left(23^{\circ} \mathrm{C}\right)$, pressure $(1060 \mathrm{mb})$, and gas mix (normal $\mathrm{N}_{2} / \mathrm{O}_{2}$ ) but illuminated with a Mars-normal UV-VIS-NIR light environment were reduced by over $99.5 \%$ after $15 \mathrm{~s}$ exposure to Mars-normal UV fluence rates. However, it required at least $15 \mathrm{~min}$ of Mars-normal UV exposure to inactivate $100 \%$ of the bacterial populations. The longer times required for complete inactivation of spores of $B$. subtilis are believed to be due to the presence of very low numbers of small clusters of bacterial spores on the aluminum coupons in which some spores provide a moderate level of protection from down-welling UV light to adjacent or underlying spores. Direct observations of the bacterial monolayers using high-resolution video imaging indicated that the overwhelming majority of the endospores existed as individual spores that were randomly distributed in smooth and uniform layers. However, it was impossible to scan each and every bacterial spore on the hundreds of monolayers used in these studies. Thus it is likely that a few small clusters of spores were involved in extending bacterial survival between $15 \mathrm{sec}$ and $15 \mathrm{~min}$ of UV irradiation. These results were duplicated when populations of $B$. subtilis were exposed to Marsnormal environments of temperature $\left(-10^{\circ} \mathrm{C}\right)$, pressure $(8.5 \mathrm{mb})$, gas composition (pure $\mathrm{CO}_{2}$ ), and UV fluence rates. In other experiments, results indicated that the gas composition of the atmosphere and the temperature of the monolayers at the time of Mars UV exposure had no effects on the survival of bacterial spores.

The results of studies using neutral density filters to simulate a firstorder approximation of UV attenuation on Mars due to dust loading in the atmosphere indicated bacterial survival was only slightly reduced up to simulated optical depths of 2.5. It was not until the simulated optical depth reached 3.5 (the average optical depth achieved on Mars during several global dust storms) did bacterial survive at significantly higher numbers than they did under a normal clear-sky condition of $\tau=0.5$.

During the course of this research, each experimental operation had a set of controls kept outside and a second set of controls kept inside the MSC system. These controls were kept shielded from all UV-VIS-NIR irradiation, and, thus, any differences between these populations would indicate overall effects of being exposed to Mars-normal versus Earthnormal conditions in which UV irradiation is not a factor. Results were very consistent among four different experiments in which the bacterial populations kept inside the MSC were reduced by $20 \%$ from those maintained outside the MSC. The primary difference between the Earthnormal and Mars-normal controls was pressure. Although other factors were involved (i.e. differences in gas composition and temperature), these effects were shown in previous experiments not to affect bacterial survival.

The implications of these results for spacecraft sanitation and sterilization procedures are significant. First, greater than $99.5 \%$ of bacterial populations on exposed surfaces of spacecraft are likely to be inactivated within a few seconds to a few minutes on the surface of Mars. Furthermore, if a 4-decade reduction in bacterial populations on spacecraft landed on Mars can be expected within approximately 15 minutes, then we would predict that within a single Mars day under clear-sky conditions the bacterial populations on most exposed surfaces would be completely sterilized. Future research will seek to model UV attenuation of the Martian atmosphere due to increasing dust loads, the effects of diffuse versus direct UV irradiation, and to simulate with higher fidelity bacterial survival on Mars under a variety of mission scenarios and landing sites.

\section{Reactive nitrogen in volcanic plumes on early Mars} Antigona Segura • Instituto de Ciencias Nuclares, UNAM, Rafael Navarro-Gonzalez $\bullet$ Instituto de Ciencias Nucleares, UNAM

The volcanic environment of early Mars was reproduced experimentally in order to determine the role of volcanic lightning in the production of reactive nitrogen, a key element for life. Volcanic lightning may have been produced during explosive eruptions that were common in Mars as is suggested by numerical simulations and observational evidence. The volcanic gas composition used in this study was derived from an accretional model of Mars (1) and the carbon-nitrogen ratio from the Martian meteorites (2). Volcanic lightning was simulated by a hot plasma produced by focusing a high-energy infrared laser in a closed flask that contained the gas mixture. The analysis of products was performed using a gas chromatograph coupled to an infrared detector and a quadrupole mass spectrometer. The products detected were hydrocarbons and hydrogen cyanide $(\mathrm{HCN})$, the only nitrogenated compound. $\mathrm{HCN}$ is a key molecule for prebiotic chemistry because it forms amino acids and nucleic acid bases. The amount of energy available in volcanic lightning was calculated to be $8 \times 10^{17} \mathrm{~J} \mathrm{yr}^{-1}$, which may have produced about $10^{11} \mathrm{~g} \mathrm{yr}^{-1}$ of $\mathrm{HCN}$. This value is compared with other possible sources of reactive nitrogen from endogenous and exogenous sources in early Mars.

\section{References}

${ }^{1}$ Kuramoto, K. (1997). Phys. Earth Planet. Int. 100, 3.

2 Wright, I.P. et al. (1992). Geochem. Cosmochem. Acta 56, 817.

\section{High throughput approach to study microbial phylogenetic diversity in permafrost as a terrestrial model of Mars}

Elena V. Spirina $\bullet$ Michigan State University; Institute of PhysicalChemical and Biological Problems of Soil Science, RAS, Pushchino, Russia, James R. Cole $\bullet$ Michigan State University, Benli Chai $\bullet$ Michigan State University, David A. Gilichinsky $\bullet$ Institute of PhysicalChemical and Biological Problems of Soil Science, RAS, Pushchino,

Russia, James M. Tiedje • Michigan State University

Since Mars is cryogenic, terrestrial permafrost soil, which is inhabited by cold-adapted microbes, is a potential extraterrestrial model for investigating new approaches to study those microbes, and to interpret biogeochemical evidence preserved in the geological record. Two distinct environments were examined: Arctic and Antarctic permafrost. To assess microbes living in this environment, 16S rDNA genes were amplified from permafrost DNA extracted both from frozen soil and those that had been incubated under both aerobic and anaerobic conditions. High-throughput DNA sequencing was performed on the cloned PCR products to obtain partial 16S rDNA gene sequences. A script was written to automatically compare over 2000 partial sequences with those rrn sequences in the Ribosomal Database Project (RDP) release 8.1 using the SEQUENCE MATCH. Sequences were grouped into categories from the RDP's phylogenetic hierarchy based on the closest database match. The results suggest significant microbial diversity in all samples. However, in both regions the diversity of organisms in incubated soil was less than detected originally. Similar phylogenic groups appeared in both polar regions and some of these phylotypes have members that are known to live in other environments. The phylotypes identified here will be important organisms to study to learn their adaptations to cryogenic conditions.

\section{Search for spectral signatures of life at the Pathfinder landing site}

Carol Stoker $\bullet$ NASA Ames Research Center, Pascal Ashwanden • NASA Ames Research Center

The Superpan, an image product from the Pathfinder lander camera, is a multispectral panorama of the Pathfinder landing site acquired in 15 wavelengths in the spectral range 440-1100 $\mathrm{nm}$. We have performed an automated search of the Superpan image cubes for the spectral signature associated with chlorophyll. First, images were calibrated to radiance values and then the multispectral images were co-registered to subpixel accuracy. An automated pixel-to-pixel search was performed on a three-filter set of images $(530,670,980 \mathrm{~nm})$ to identify pixels where the following condition was met: $530 \mathrm{~nm}>670 \mathrm{~nm}$ and $980 \mathrm{~nm}>670 \mathrm{~nm}$. Thus, we searched for the spectral signature associated with red light absorption by chlorophyll. When this case was met by the search routine, we plotted a full spectrum for the pixels involved and carefully examined the images. The condition was met for small areas in six image 
cases. All of these cases occur in near-field images, where the resolution is highest. Four of the cases occur on the spacecraft and appear to be associated with spacecraft structure. Two intriguing cases occur in small areas on the ground near the spacecraft.

\section{Biological iron isotope fractionation in the Antarctic cryptoendolithic microbial community}

Henry Sun $\bullet$ Jet Propulsion Laboratory, Brian Beard $\bullet$ University of Wisconsin, Clark Johnson • University of Wisconsin, Joe Skulan University of Wisconsin, Imre Friedmann • NASA Ames Research Center, Kenneth Nealson • University of Southern California

The composition of stable isotopes of iron is a potentially powerful biomarker because iron is ubiquitous in the solar system and it is required for the growth of all organisms on Earth. Since the initial report of Beard et al. (1999) that the iron-reducing bacterium Shewanella can fractionate iron isotopes in the laboratory, there have been many attempts to link biological activities and variations in the iron isotope composition in the natural environments. However, most studies have focused on aquatic systems and are confounded by the fact that aquatic chemistry without life can also fractionate iron isotopes. Here we report evidence of biological iron isotopic fractionation in a naturally occurring microbial community under the surface of exposed sandstone in McMurdo Dry Valleys, Antarctica. Microbially produced oxalic acid leaches out iron oxides from the colonized layer, forcing the iron to accumulate in the zone below. This photo-reductive dissolution process appears to preferentially mobilize lighter iron isotopes, leaving the colonized layer enriched in $\mathrm{del}^{56} \mathrm{Fe}$ by as much 0.8 per mil. If endolithic microorganisms also occurred on Mars as commonly believed, they might have left similar iron biosignatures, which would be preserved in rocks because of the absence of subsequent aqueous activities.

\section{The geochemistry of Atacama desert soils: a possible Mars analog \\ Brad Sutter $\bullet$ National Research Council; NASA Ames Research Center, Ron Amundson • University of California, Berkeley, Stephanie Ewing • University of California, Berkeley, Kim Warren-Rhodes • National Research Council, NASA Ames Research Center, Chris Mckay $\bullet$ NASA Ames Research Center}

The Atacama Desert of northern Chile is possibly the oldest and driest desert in the world, and is one of the best terrestrial Mars analogs for developing hypotheses about the geochemistry of the Martian surface. The objective of this work was to examine the accumulation and processing of elements in soils of the hyper-arid Atacama Desert to provide insight into what soil properties may be found on Mars.

Three soil sites were established along a south to north transect where precipitation decreases from 15 to $\sim 0 \mathrm{~mm} \mathrm{yr}^{-1}$. Soils were sampled for mineralogical and chemical assays and meteorological stations and atmospheric collectors were installed. Aeolian inputs of silicate and more soluble minerals appears to be an important process in all soils based on surface horizon characteristics. Soil $\mathrm{pH}$ values decreased from 7.8-8.3 to 6.9-7.7 along the transect, reflecting changes in soil chemistry from carbonate to nitrate $\left(\mathrm{NO}_{3}^{-}\right)$dominated systems. Soil solution nitrate concentrations in the northern soil ranged from $810-17633 \mathrm{mg}$ $1^{-1}$. The $\mathrm{NO}_{3}^{-}$concentrations did not exceed $180 \mathrm{mg} \mathrm{NO}_{3}^{-} \mathrm{L}^{-1}$ at any of the other sites. The large concentration of salts $\left(\mathrm{NO}_{3}^{-}\right.$and $\left.\mathrm{SO}_{4}^{2-}\right)$ in all soils indicates long-term aridity, but discontinuities in soil horizons and the presence of variable amounts of $\mathrm{CaSO}_{4}$ at the surface of all three soils indicates that the region is now in an exceedingly arid phase.

\section{Archaeoglobus fulgidus: an ideal microorganism for studying Martian carbonate precipitation}

K.A. Van Cleave • University of South Florida, L.L. Robbins $\bullet$ US Geological Survey

Several attempts have been made to produce carbonates similar to those found in the Martian meteorite ALH 84001, by inorganic processes (Golden et al. 2000; Socki et al. 1999; Golden et al. 2001). However, no attempts have been made thus far to produce carbonates like those found in ALH 84001 via biological means. Using the hyperthermophilic, anaerobic, vent-dwelling archaeon Archaeoglobus fulgidus, we produced carbonates chemically, morphologically and isotopically similar to those present in ALH 84001.

Carbonates precipitated by $A$. fulgidus have filamentous, globular and ovoid morphologies and range in size from 2.5 to $23 \mu \mathrm{m}$ in longest dimension. The composition of the carbonates is ferroan magnesite with minor amounts of dolomite, cerussite, rhodochrosite and strontianite. Precipitation of carbonates with A. fulgidus cells in a ${ }^{13} \mathrm{C}$ enriched system resulted in carbonates with $13 \mathrm{C}$ values as high as those in ALH 84001 carbonates $(+48.1$ to $+59.8 \%$ ). Precipitation using inorganic processes has yet to produce such ${ }^{13} \mathrm{C}$-enriched carbonates. References

Golden, D.C. et al. (2000). Meteoritics \& Planetary Science 35, 457-465. Golden, D.C. et al. (2001). American Mineralogist 86, 370-375.

Socki et al. (1999). Abstracts with Programs - Geological Society of America 31, 339.

\section{Evaporites in Martin meteorites: traces of Martian history}

Susan J. Wentworth $\bullet$ Lockheed Martin Space Operations, David S. McKay $\bullet$ NASA Johnson Space Center, Michael A. Velbel $\bullet$ Michigan State University, Kathie L. Thomas-Keprta $\bullet$ Lockheed Martin Space Operations, Teresa G. Longazo $\bullet$ Hernandez Engineering

Aqueous activity may have produced many of the erosional and depositional features seen on Mars by remote sensing, at least at some time(s) in the past. Several Martian meteorites contain evaporite minerals and other aqueous alteration products which probably formed on Mars. The Martian secondary environment apparently contained many components critical for life on Earth; e.g. circulating water, organic phases, and temperatures overlap the range compatible with those known to support biogenic activity on Earth for organisms. The wide range in Martian meteorite ages suggests that conditions on Mars may have been favorable for life for a large part of geological history. Field emission SEM (FE-SEM) and energy-dispersive X-ray spectroscopy (EDS) of Martian meteorites continue to reveal new evidence for low-temperature Martian aqueous activity and the development of evaporites on Mars. We are also evaluating previously obtained data in order to determine with what certainty we can assign specific minerals and assemblages to either Earth or Mars. It is important to do extensive, detailed studies of the meteorites because of the scarcity of possible Martian alteration products. Because evaporite mineral abundances are low in Martian meteorites, the planet of origin for individual evaporite occurrences is determined by close examination of their petrographic associations. For example, partly devolatilized Ca-sulfate inclusions within the fusion crust of the Nakhla meteorite provide unambiguous proof that the sulfate formed on Mars. Without having pristine samples from Mars, distinguishing between Martian and terrestrial features in the Martian meteorites is a critical part of understanding both the nearsurface history of Mars and conditions that may have supported biological activity in the past. If life existed on Mars in the past, traces of it may be closely associated within evaporites, just as biogenic features are preserved and fossilized in terrestrial evaporites.

\section{Prospecting for habitat beneath the Martian north polar cap using radar: inferences from terrestrial experience}

Dale P. Winebrenner $\bullet$ University of Washington

The search for life elsewhere in our solar system often reduces to a search for liquid water. Water may occur at depth in the Martian crust, but neither solid nor liquid water has thus far been definitively observed there. The Martian north polar cap, by contrast, certainly consists largely of water ice in a mass roughly $3 \mathrm{~km}$ thick, thus its base is an especially interesting place to probe for liquid water. Sounding radars are routinely used to probe terrestrial ice for areas of basal wetness, based on stronger reflections from wet beds than from frozen ones. Estimation of bed reflectance from the vantage of the ice sheet surface, however, is complicated by large uncertainties in the attenuation of signals propagating to the bed and back. Direct observational charac- 
terization of attenuation in the ice therefore strongly supports radar investigation of bed wetness on Earth. On Mars, where the mean and variability of ice temperature and impurity burdens are almost unknown, direct observations of attenuation become even more important. Here, I present radar data from West Antarctica together with analysis that provides an estimate of attenuation in the ice. I show how similar measurements could be obtained by placing one or more receivers on the Martian north polar cap during the times that orbital sounding radars that are currently planned for Mars are in operation.

\section{Evolution and detection of hydrothermal refugia in the Martian subsurface}

Aaron Zent $\bullet$ NASA Ames Research Center

Subsurface hydrothermal systems are often invoked as potential refugia for subsurface Martian biota. Their detection, particularly through remote sensing, is a high-priority Mars exploration goal.
The Martian regolith is cold, and water vapor rising from a subsurface hydrothermal system will freeze, making an impermeable self-sealing ice cap. The position of the ice-vapor interface is an energy balance problem amenable to numerical study.

If too much heat is delivered to the ice interface, ice will melt and the interface moves toward the surface. If too little heat is delivered, the vapor will condense and freeze, moving the interface downward. When equilibrium is achieved, rising vapor will condense to liquid at the ice interface, deposit its latent heat of fusion there, and then drain as liquid.

Critical variables are surface temperature, temperature of water, and the relative depth of the ice and liquid interfaces. If the net heat flux to the ice interface is greater than the ice cap can conduct away (maximum approximately $10 \mathrm{~W} \mathrm{~m}^{-2}$ ) the system will break through to the surface. A sealed subsurface hydrothermal system can be stable, but will trap metabolic waste. It will be very hard to detect in the atmosphere. An open subsurface hydrothemal system will be easier to detect, mostly by vapor escape, but will lose molecules irreversibly to the polar cold trap.

\section{Europa}

\section{A new energy source for organic synthesis in Europa's ice}

Jerome Borucki $\bullet$ NASA Ames Research Center, Bishun Khare • SETI Institute, Dale Cruikshank $\bullet N A S A$ Ames Research Center

Colored regions on Jupiter's satellite Europa and other icy bodies in the outer Solar System may be contaminated by organic macromolecular solid material that is produced when surface ices are exposed to electrical energy. Hypervelocity meteorite impacts and fracture release tidal and tectonic stresses in icy crusts in the form of electrical discharges, which provide the energy for in situ synthesis of the organic solids. We report measurements of electrical discharge, light emission, and magnetic phenomena in hypervelocity impacts into ice with projectiles with $\mathrm{V}$ $\sim 5 \mathrm{~km} \mathrm{~s}^{-1}$. Part of the projectile's kinetic energy is converted into electrical potential, while the mechanical disruption of the impact also releases stress energy as light, heat, electrical, and magnetic fields as secondary emissions. These newly recognized energy sources suggest that the dark material in the area of impact craters are tholins generated from the energy of the impacts and that well up from the fracture zone. Large pools of liquid water would persist under the meteorite crater for thousands of years (Thomson \& Sagan 1992), with the potential for prebiotic chemistry to take place at an accelerated rate due to energy pumped in from the secondary emissions.

\section{Radiolytic degradation of molecular biosignatures} Robert W. Carlson • Jet Propulsion Laboratory, California Institute of Technology, Mark S. Anderson • Jet Propulsion Laboratory, California Institute of Technology

Astrobiological missions to Europa are being studied by the Astrobiology Institute's Europa Focus Group, who recommended chromatographic and spectroscopic searches for biomarker molecules that may be present in the near subsurface. Europa is bombarded by high-energy electrons and protons that rapidly decompose and chemically modify surface materials, so the stability and alteration of biomarker molecules is of interest. We have initiated radiation studies of representative biosignature molecules under Europan irradiation conditions and are employing gas chromatography and mass spectroscopy to determine the radiolysis products. Initial studies are being performed on pure picolinic acid and cholesterol and their aqueous solutions. A variety of molecular products are anticipated, formed from molecular dissociation, dimerization, polymerization, and addition and abstraction reactions. Results for a 10 Mrad dose will be presented.

\section{The infrared signature of Europa salts}

Galina Chaban $\bullet N A S A$ Ames Research Center, Winifred Huo $\bullet$ NASA Ames Research Center, Timothy Lee $\bullet$ NASA Ames Research Center Europa has attracted much attention from the planetary scientists and astrobiologists because of its possible subsurface ocean of water. In addition, data from Galileo's near-infrared mapping spectrometer (NIMS) indicate the possibility of hydrated salts on its surface. To aid in the design of future missions, we investigated the infrared signature of $\mathrm{MgSO}_{4}-n \mathrm{H}_{2} \mathrm{O}(n=1-3)$ using ab initio calculations. Geometry, energetics, dipole moments, as well as vibrational frequencies and infrared intensities of pure and hydrated $\mathrm{MgSO}_{4}$ salts were determined. For the pure $\mathrm{MgSO}_{4}$ and its complex with one water molecule, anharmonic corrections to vibrational spectra were computed. Our calculations showed that the vibrational spectrum of water complexed with $\mathrm{MgSO}_{4}$ is significantly different from the pure water spectrum. For each complex, there is at least one conformer with the $\mathrm{O}-\mathrm{H}$ stretch frequency shifted to the red by up to $1500-2000 \mathrm{~cm}^{-1}$. The intensity of that band becomes approximately an order of magnitude larger than the corresponding value in pure water. In addition, the $\mathrm{SO}_{2}$ stretch frequencies are found to be lower than the water frequencies and their intensities to be about two to three times stronger than the normal water stretch intensity. The calculated bands of water and $\mathrm{SO}_{2}$ fragments can serve as markers for the existence of salt-water complexes on the surface of Jupiter's moon.

\section{Chemical, physical, and biological environment of Europa's floating shell, ocean, and subocean crust Jeffrey S. Kargel $\bullet$ U.S. Geological Survey, Olga Prieto $\bullet$ Centro de Astrobiologia, Madrid, Spain, Jonathan Z. Kaye $\bullet$ University of Washington, Seattle, Steve Vance $\bullet$ University of Washington, Seattle, Giles Marion • Desert Research Institute, David L. Hogenboom • Lafayette College, Easton, $P$ A}

Europa's early hydrosphere probably was saturated in $\mathrm{Mg}$-Na-sulfates. Its ocean may have evolved either an acidic or alkaline character. Either way, now it should be a high-pressure eutectic brine, including $\mathrm{C}-\mathrm{H}$ compounds. The floating shell is probably salty ice. The seafloor crust may consist of tens of kilometers of bedded salts. Radiogenic and tidal heating in the silicate core may drive metamorphic dehydration of salts in the upper few kilometers of suboceanic crust. Cavernous structures eroded into the seafloor deposits may contain dense, warm, hypersaline brines. The seafloor and subseafloor crust is probably hypersaline throughout, and may be thermally diverse, including some niches of ordinary temperature and others suited for psychrophilic through hyperthermophilic life. On Earth, polar and geothermal environments harbor diverse cold-loving and heat-loving communities, respectively. Each class of microorganism has evolved adaptations to grow at extreme temperatures. For example, hyperthermophiles synthesize organic thermoprotective compounds and small proteins to protect macromolecules within a cell. Extrinsic factors such as pressure may also help cells survive. Some psychrophiles and hyperthermophiles are moderately halotolerant, but little is known about the fundamental limits of such organisms in chemophysical conditions as extreme as some models of Europa's ocean. 
Laboratory support for a methanogenesis driven biosphere in Europa

Rafael Navarro-Gonzalez $\bullet$ Universidad Nacional Autonoma de Mexico, Lilia Montoya $\bullet$ Universidad Nacional Autonoma de Mexico, Wanda Davis $\bullet$ SETI Institute, Christopher McKay $\bullet$ NASA Ames Research Center

Methanogenesis associated with hydrothermal vents may potentially drive a biosphere in a Europan ocean. Here we explore this possibility experimentally by simulating a hydrothermal vent relevant to Europa and analyzing the resulting gas products by gas chromatography coupled to Fourier transform infrared and mass spectrometries.

The chemical composition of the hydrothermal vent gases was derived from a thermochemical model that assumes Europa had a cometary (solar, less $\mathrm{H}$ ) abundance at high temperatures characteristic of a vent. Specifically the following gas mixture was used: $45 \% \mathrm{CO}_{2}, 45 \% \mathrm{CH}_{4}$, and $10 \% \mathrm{~N}_{2}$. A $500 \mathrm{ml}$ stainless steel reactor was filled with $200 \mathrm{ml}$ triply distilled water and the gas mixture at 1 bar at $25^{\circ} \mathrm{C}$. In some experiments $3 \mathrm{~g}$ of pyrite were added into the reaction vessel. The system was heated for $24 \mathrm{~h}$ in the temperature range from 100 to $375^{\circ} \mathrm{C}$. In another series of experiments the oxidation state of carbon was also varied from $100 \%$ methane to $100 \%$ carbon dioxide. In all cases, a suite of complex hydrocarbons was formed. Our results support two possible forms of methanogenesis in Europa driven by: (1) reduction of $\mathrm{CO}_{2}$ to $\mathrm{CH}_{4}$ using $\mathrm{H}_{2}$; and (2) cleavage of hydrocarbons back to $\mathrm{CH}_{4}$.

\section{Detection and characterization of extrasolar planets}

\section{The galactic exoplanet survey telescope}

David Bennett $\bullet$ University of Notre Dame, Ed Cheng $\bullet$ Goddard Space Flight Center, Kem Cook $\bullet$ LLNL, Jonathan Lunine $\bullet$ University of Arizona, Sun Hong Rhie $\bullet$ University of Notre Dame, Nick Woolf • University of Arizona

We show that a space-based gravitational microlensing survey for terrestrial extra-solar planets is feasible in the near future. Such a survey could provide a nearly complete picture of the abundance and mass function planetary systems in our Galaxy. A space-based 1-2 m telescope with a $\sim 2$ square degree field of view would be used to continuously monitor $\sim 100$ million Galactic bulge main sequence stars in order to detect the photometric signatures of terrestrial planets with masses as low as $1 / 10$ of an Earth mass. Microlensing is unique in its ability to detect terrestrial planets with high signal-to-noise ratio, and it has it is able to detect planets with the same mass and separation as all of the Solar System's planets except for Mercury and Pluto. It is complementary to the transit technique because it is sensitive to planets at all separations $>0.7 \mathrm{AU}$. A space-based survey will also be able to detect the planetary host stars for most of the planets that orbit solartype stars. This enables the determination of the planetary masses and separations in physical units, as well as the abundance of planets as a function of stellar type and distance from the Galactic center.

\section{Kepler Mission: the first step in determining the extent of life in the Universe}

William Borucki $\bullet$ NASA Ames Research Center, David Koch $\bullet$ NASA Ames Reseach Center, Gibor Basri $\bullet$ University of California, Berkeley, Edward W. Dunham $\bullet$ Lowell Observatory, Ronald Gilliland $\bullet$ STScI, David Latham $\bullet$ Smithsonian Astrophysical Observatory

The first step in discovering the extent of life in our galaxy is to determine the number of terrestrial planets in the habitable zone (HZ). The Kepler Mission is a $0.95 \mathrm{~m}$ aperture photometer scheduled to be launched in 2006. It is designed to continuously monitor the brightness of 100000 solar-like stars to detect the transits of Earth-size and larger planets. The depth and repetition time of transits provide the size of the planet relative to the star and its orbital period. When combined with ground-based spectroscopy of these stars to fix the stellar parameters, the true planet radius and orbit scale, hence the relation to the $\mathrm{HZ}$ are determined. These spectra are also used to discover the relationships between the characteristics of planets and the stars they orbit. In particular, the association of planet size and occurrence frequency with stellar mass and metallicity will be investigated. Based on the results of the current Doppler-velocity discoveries, over a thousand giant planets will be found. Information on the albedos and densities of those giants showing transits will be obtained. At the end of the 4 year mission, hundreds of terrestrial planets should be discovered in and near the $\mathrm{HZ}$ of their stars if such planets are common. A null result would imply that terrestrial planets in the $\mathrm{HZ}$ occur in less than $1 \%$ of the stars and that life might be quite rare.

\section{Increasing the payoff of ground-based exoplanet transit searches}

Douglas Caldwell $\bullet$ SETI Institute, Natalie Batalha $\bullet$ NASA Ames

Research Center, William Borucki $\bullet N A S A$ Ames Research Center, Jon Jenkins $\bullet$ SETI Institute, David Latham $\bullet$ Harvard; Smithsonian Center for Astrophysics, Zoran Ninkov $\bullet$ Rochester Institute of Technology

Observations of the one known transiting exoplanet (HD 209458b) have resulted in an abundance of valuable science and increased the pressure to find more transiting planets. With current photometric techniques, sufficient precision to detect terrestrial size planets (radii $\leqslant 2 \mathrm{R}_{\text {Earth }}$ ) around $\mathrm{M}$-dwarf stars can be obtained. However, even with several groups working for the past few years, there has yet to be a confirmed exoplanet discovery resulting from a transit search. We will detail efforts being undertaken at NASA Ames to move towards detecting terrestrial size planets and to increase the detection rate of transiting giant exoplanets. These efforts include work on several fronts. We are tailoring the data analysis to increase photometric precision of the most numerous faint stars. We are selecting star fields and ranges of stellar magnitudes to observe to maximize the number of useful target stars. We are working to optimize observing sites and procedures to increase transit detection rates. We have developed a systematic approach for following up on candidate detections that includes assessing their statistical significance, difference-image photometry to eliminate background confusion, medium-resolution radial velocity measurements to eliminate stellar mass companions, and finally high-resolution radial velocity measurements to confirm planetary mass candidates.

\section{Kepler Mission: accentuate the positive, eliminate the false positive}

John Caldwell • York University, Christopher Ryan • York University, William Borucki $\bullet$ NASA Ames Research Center, David Koch $\bullet$ NASA Ames Research Center

Kepler is a NASA mission that will search for Earth-like planets orbiting Sun-like stars. Its goals and philosophy have been described elsewhere (W. Borucki, D. Koch, et al. this meeting). Among the selfimposed criteria for designing this mission, Borucki et al. have decided that if the true number of exoEarths is small or zero, such a finding must be statistically significant. It follows that all sources of possible false positive discoveries must be vigorously considered. One potential problem-possible confusion from background stars, particularly eclipsing binaries near the line of sight to a foreground target star - is addressed here. Because high photometric precision is required to detect a transiting exoEarth, Kepler's optics are intentionally defocussed to minimize photometric noise from spacecraft jitter. This increases the risk of degraded photometric accuracy due to background stars. Further, HST images within the Kepler target area show that the number of confusion sources is significant. The special concern about binary stars, which constitute half of all background sources, is that if 
they are eclipsing, their temporal profiles mimic a planetary transit, especially in low signal-to-noise ratioevents. The risk can be mitigated by preserving all the information in all pixels and by examination of the stars with ground-based telescopes and HST.

Tidal heating and 51 peg-type planets

Will Clarkson • University of Southampton, Bojan Pecnik • Max Planck Institut für Astrophysik

With a star-planet separation of $0.05 \mathrm{Au}$, short-period planets like that detected in the 51 Peg system are close enough to the parent star for a gas giant to half-fill its Roche lobe, deforming it in the process. Tidal dissipation in such systems will cause the planet to assume a circular orbits and corotation on astronomically short timescales. The circularization process leads to significant tidal heating, the dissipation of which depends sensitively on the transport processes at work within the planet, and thus on its internal structure. We examine the effect of the tidal heat source on the evolution of a variety of star-planet systems, including scenarios involving planetary migration. In the case of $51 \mathrm{Peg}$ itself, in which the parent star is thought to rotate significantly more slowly than corotation would demand, the possibility exists that the spin-up of the parent star by the orbiting planet will cause the planet to migrate further towards the parent star.

\section{Remote sensing of planetary properties and biosignatures on extrasolar terrestrial planets}

David J. Des Marais $\bullet$ NASA Ames Research Center, Martin Harwit $\bullet$ Cornell University, Kenneth Jucks $\bullet$ Harvard-Smithsonian Center for Astrophysics, James Kasting • Pennsylvania State University, Douglas Lin • University of California, Santa Cruz, Jonathan Lunine • University of Arizona, Jean Schneider $\bullet$ Observatoire de Paris, Sara Seager $\bullet$ Institute for Advanced Study, Princeton, NJ, Wesley Traub • Harvard-Smithsonian Center for Astrophysics, Neville Woolf $\bullet$

University of Arizona

The major goals of NASA's Terrestrial Planet Finder (TPF) and ESA's Darwin missions are to detect terrestrial-sized extrasolar planets directly and to seek spectroscopic evidence of habitable conditions and life. Here we recommend wavelength ranges and spectral features for these missions. We assess known spectroscopic features of Earth, Venus and Mars in the context of putative extrasolar analogs. For the mid-IR and the visible to near-IR, the preferred wavelength ranges are 7-25 microns and 0.5 to about 1.1 microns, respectively. Detection of $\mathrm{O}_{2}$ or its photolytic product $\mathrm{O}_{3}$ merits highest priority. Liquid $\mathrm{H}_{2} \mathrm{O}$ is not a bioindicator but it is considered essential to life. Substantial $\mathrm{CO}_{2}$ indicates an atmosphere and oxidation state typical of a terrestrial planet. Abundant $\mathrm{CH}_{4}$ might require a biological source, yet abundant $\mathrm{CH}_{4}$ can also arise from a crust and upper mantle more reduced than that of Earth. The range of characteristics of extrasolar rocky planets might far exceed that of the Solar System. Planetary size and mass are very important indicators of habitability and can be estimated in the mid-IR, and potentially also in the visible to near-IR. Both wavelength ranges offer valuable information regarding biosignatures and planetary properties; therefore both merit serious scientific consideration for TPF and Darwin.

\section{Prospects for detection and characterization of terrestrial planets in stellar habitable zones using reflected light}

Dennis Ebbets $\bullet$ Ball Aerospace and Technologies Corp., Steve Kilston - Ball Aerospace and Technologies Corp.

During our NASA sponsored study of candidate architectures for the Terrestrial Planet Finder mission we estimated the values of observable properties that would be available to an instrument intended to detect starlight reflected by a planet in the habitable zone of the system. These properties include architecture- and wavelength-independent geometrical properties such as angular separation between the star and planet, and timescales associated with orbital motion. Properties that do depend on the detection technique and wavelength include the brightness of the planet, its contrast relative to the star, and variability associated with diurnal and seasonal phenomena. The search space for a reflected light
TPF is the range of these parameters calculated for a sample of 200 main sequence stars whose stellar properties make them attractive potential targets. The desire to perform a scientific investigation such as that described by the TPF Science Working Group then leads to requirements on the sensitivity of the system, angular resolution, suppression of starlight and operational efficiency. We will describe the rationale for selection of our star sample, the search space of planetary observables, apparent system requirements, and our assessment of the feasibility of this approach.

\section{Migration of solids in a gravitationally unstable disk;} a possible scenario for terrestrial planet formation Nader Haghighipour $\bullet$ Carnegie Institution of Washington, DTM, Alan Boss $\bullet$ Carnegie Institution of Washington, DTM

Theoretical models of the formation and evolution of the solar nebula provide the context for understanding the early stages of collisional growth of dust grains that eventually lead to the formation of planets. Two mechanisms have been proposed to describe the formation of gas giant planets: the disk instability model and the core accretion scenario. It has recently been realized that in both of these mechanisms, a solar nebula capable of forming gas giant planets is likely to be marginally gravitationally unstable.

Considering either the core accretion or the disk instability model, it will be fundamentally important to investigate the implications for the collisional aggregation and orbital evolution of small bodies, ranging from dust grains to planetesimals, orbiting in such an unstable disk. This has motivated us to launch a systematic and thorough study of the dynamical evolution of small bodies in a marginally gravitationally unstable disk.

We present the results of a numerical study of the orbital evolution of small bodies, subject to Epstein and Stokes drags as well as the selfgravity of the disk, in the terrestrial planet region of a marginally gravitationally unstable model of the solar nebula. We show that as a result of deviation of the orbital velocities from Keplerian values, bodies at both sides of the location of a peak in the radial density distribution undergo radial migration toward the central peak. The implications of such migrations for collisional coagulation and possibly formation of terrestrial planets are discussed.

\section{Accumulation of giant planet atmospheres around cores of a few Earth masses}

Olenka Hubickyj • University of California, Santa Cruz; NASA Ames Research Center, Peter Bodenheimer • University of California, Santa Cruz, Jack J. Lissauer $\bullet$ NASA Ames Research Center

The widely accepted core instability model states that gas giant planets form by accretion of a massive solid core from the planetesimals in the solar nebula followed by the capture of a massive envelope from the solar nebula gas. Our simulations based on this model have been successful in explaining many features of the giant planets. Recent interior models of Jupiter and Saturn suggest smaller solid core masses that have been predicted by previous formation models. We have computed simulations of the growth of Jupiter where we vary the grain opacity and the planetesimal surface density in the solar nebula. We also explore the implications of halting the solid accretion at selected core mass values during the protoplanet's growth. Our results show that reducing grain opacities reduces the formation time scale and that decreasing the surface density of planetesimals lowers the final core mass of the protoplanet but increases the formation time scale. Derived core masses and observed short lifetimes of protoplanetary disks strongly constrain conditions for forming gas giant planets. The key to satisfying the constraints appears to be a grain opacity substantially less than the interstellar value, consistent with recent calculations of grain settling in giant planet atmospheres.

\section{An adaptive, wavelet-based method for detecting transiting extrasolar planets \\ Jon Jenkins $\bullet$ SETI Institute}

The Kepler Mission was recently selected by NASA's Discovery Program for launch in 2006. Kepler's goal is to search for planets 
transiting solar-like stars. Kepler will stare at the same starfield for at least 4 years, tracking the brightnesses of over 100000 stars continually and simultaneously during this time. The noise on the photometric lightcurves will consist of several components, several of which can be limited by careful design of the spacecraft, the instrument and the operation of the CCD arrays. Stellar variability, however, cannot be controlled by design, and must be dealt with on its own terms. Studies of solar irradiance by instruments such as DIARAD aboard the SOHO spacecraft demonstrate that solar-like variability is a time-varying noise process. Here we detail an adaptive, wavelet-based detection algorithm tailored for detecting transiting planets for the Kepler Mission, and demonstrate it for synthetic data consisting of the DIARAD time series plus additional noise representing shot and instrument noise for Kepler. The results show that solar-like variability does not prevent the detection of Earth-sized planets even for stars rotating significantly faster than the Sun. Indeed, the fundamental limits represented by solar-like variability allow for the detection of planets significantly smaller than Earth orbiting Sun-like stars.

\section{Coupled radiative-photochemical calculations of the rise of atmospheric $\mathrm{O}_{2}$}

Kara Krelove $\bullet$ Pennsylvania State University, James Kasting • Pennsylvania State University

Oxygen, and therefore ozone, is considered a good preliminary marker for biological activity on extraterrestrial planets. Furthermore, ozone has a strong absorption band at 9.6 microns, making it an excellent target for IR spectrum observations, such as those anticipated for the Terrestrial Planet Finder (TPF) mission. (1) The shape and size of the absorption spectra is influenced by $\mathrm{O}_{3}$ concentrations as well as the temperature in the stratosphere.

Calculations of $\mathrm{pO}_{3}$ as a function of $\mathrm{pO}_{2}$ has been done previously by Schindler \& Kasting ${ }^{1}$ but the temperature profiles in that model were not computed self-consistently. Here we correct that problem. In our coupled model, the photochemical code is based on the work by Schindler \& Kasting. ${ }^{1}$ The temperature profiles rely on a visible/nearIR radiative-transfer (RT) algorithm from Schindler \& Kasting ${ }^{1}$ and an IR algorithm from Mlawer et al. ${ }^{2}$ Our model allows us to determine temperature profiles and species mixing ratios from approximately 0 to $65 \mathrm{~km}$, and demonstrates a good degree of accuracy for the modern Earth's atmosphere. Model calculations for ozone given varying $\mathrm{O}_{2}$ levels will be presented.

References

${ }^{1}$ Schindler \& Kasting, J (2000). Icarus 145, 262-271.

${ }^{2}$ Mlawer et al. (1997). JGR 102, D 14, 16663.

\section{Dynamics of the GJ 876 planetary system}

Jack Lissauer $\bullet$ NASA Ames Research Center, Eugenio Rivera $\bullet$ $D T M / C I W$

We present two planet fits to the radial velocity measurements of the $\mathrm{M}$ dwarf star GJ 876 obtained at the Keck observatory by Geoffrey Marcy's planet search team. Our calculations account for the mutual perturbations of planets as well as their interactions with the star. We also show the results of long-term integrations testing the stability of some of our best-fit systems. Our Newtonian models fit the data much better than do the Keplerian. Several different local minima are found in parameter space, most of which have small relative inclinations of the planets. Most sets of planetary parameters that we have derived represent systems that are stable for at least $10^{8}$ years. Test particles orbiting between the two planets are expelled from the system in less than $10^{3}$ years.

\section{Numerical simulations of gaseous protoplanetary disks: fragmentation into giant planets \\ Lucio Mayer • University of Washington, Thomas Quinn • University of Washington, James Wadsley $\bullet$ University of McMaster, Hamilton, Ontario, Canada \\ We report on results of new, high-resolution simulations of the}

formation of giant planets by the mechanism of gravitational instability in massive, cold, gaseous protoplanetary disks. We show that gaseous clumps with masses comparable or a few times bigger than Jupiter can form very quickly, on a timescale of only a few hundred years, in disks with masses close to the upper limit derived for the protosolar nebula. The degree of fragmentation depends on numerical resolution but the simulations presented here are close to the point where the results should converge to the correct physical answer. Fragmentation would prevail over gas accretion from a pre-existing planetesimal core as a mechanism to form Jupiter-like planets as the latter may require several million years. Establishing which is the actual mechanism for giant planet formation would have inevitable consequences even on the formation of terrestrial planets and eventually on the development of life; indeed an earlier or later formation of giant planets would have different effects on the dynamical evolution of planetesimals that will form terrestrial planets.

\section{Searching for the onset of grain growth in a million- year old proto-planetary disk}

Caer McCabe $\bullet$ University of California, Los Angeles, Gaspard

Duchene • University of California, Los Angeles, Andrea Ghez • University of California, Los Angeles

GG Tau A is one of the few T Tauri systems in which a proto-planetary disk has been spatially resolved at visible, near-infrared and millimeter wavelengths and is therefore an ideal system for investigating dust composition. The disk surrounds a 0.25 arcsec binary system and is both massive and geometrically thick ( 0.13 solar mass with a total height of $88 \mathrm{AU}$ at $180 \mathrm{AU}$ ). Previous NIR observations led to the suggestion that grain growth may have occurred, an important first step in the formation of planetary systems. We present high signal-to-noise ratio HST observations of the disk in the NIR and compare these measurements with Monte Carlo scattering simulations. We show that a wide range of disk properties (including disk colors that vary from blue to red) can be recreated depending on the dust model used, emphasizing the current uncertainty in ISM dust properties. We derive a model-independent lower limit on the asymmetry parameter of the dust grains, corresponding to a median grain size $a>0.2$ micron. This provides no evidence for significant grain growth in this million-year old system.

\section{The virtual planetary laboratory: toward astronomical detection of biosignatures}

Victoria Meadows $\bullet$ Jet Propulsion Laboratory; California Institute of Technology

The Virtual Planetary Laboratory (VPL) is a recently funded 5-yr project, which seeks to improve our understanding of the range of plausible environments and the likely signatures for life on extrasolar terrestrial planets. To achieve these goals we are developing a suite of innovative modeling tools to simulate the environments and spectra of extrasolar planets. The core of the VPL is a coupled radiative transfer/ climate/chemistry model, which is augmented by interchangeable modules which characterize geological, exogenic, atmospheric escape, and life processes. The VPL is validated using data derived from terrestrial planets within our own solar system. The VPL will explore the plausible range of atmospheric compositions and spectra for extrasolar planets and for early Earth, improving our understanding of the effect of life on a planet's atmosphere. We will produce a comprehensive spectral catalog, and provide recommendations on the optimum wavelength range, spectral resolution, and instrument sensitivity required for astronomical life detection. The VPL is envisioned to be a comprehensive and flexible tool, which can be collaboratively used by the broader planetary science and astrobiology communities. This presentation will describe the project concept, the tasks involved, and will outline current progress to date. This work is funded by the NASA Astrobiology Institute. 


\section{Chaotic migration of asteroids over geological timescales}

Ferenc Varadi $\bullet$ University of California, Los Angeles, Michael Ghil Dep • University of California, Los Angeles, Bruce Runnegar • University of California, Los Angeles

The orbital stability of asteroids on time scales shorter than a few million years is governed by resonances with Jupiter. We ask how stable are the supposedly stable regions on longer time scales? To do so, we are simulating at present the orbital evolution of 20 named asteroids in the regions that are stable on "short" time scales; this calculation now extends for more than 100 million years. Some asteroids appear to have very stable orbits. Others are captured into resonances with Jupiter or Mars for millions of years, but eventually are ejected from those regions when their semi-major axes jump to different values. The jumps take place in an apparently chaotic fashion and lead to random walks across the asteroid belt.

\section{Planets within the habitable zones around nearby stars}

Darren M. Williams $\bullet$ Pennsylvania State University; The Behrend College

Today 76 planets are known to orbit 67 sun-like stars, most within a radius of 50 parsecs from the Earth. The stars themselves range in spectral type from F7 to M5, and from luminosity class V (main sequence, like the Sun) to luminosity class III (sub-giants). The companions to most of these stars have been detected through careful, longterm monitoring of stellar radial velocity variations, which reveal the product $m \sin i$ ( $m$ is mass and $i$ is the inclination of the planet's orbit relative to our line of sight), not the planetary mass $m$. Thus far, the companions are all more massive than $0.16 M_{\mathrm{J}}(0.6$ times the mass of Saturn) and have average orbital radii between 0.038 and $4.47 \mathrm{AU}$ around their parent stars. Over one-third (26) of the new objects spend at least a portion of their orbits within the habitable zone (HZ) of their stars, and many on orbits with eccentricities $e>0.4$. Systems with giant planets whose orbits cross the HZ boundaries might harbor life on terrestrial-like moons if the seasonal temperature extremes resulting from the planets' large orbital eccentricities are not too severe. Here we show the distribution of extrasolar planets in relation to the $\mathrm{HZ}$ boundaries so as to highlight those systems most likely to harbor waterdependent life.

\section{References}

Extrasolar Planets Catalog. (www.obspm.fr/encycl/catalog.html). (2002).

Maintained by Jean Schneider.

Kasting, J.F., Whitmire, D.P. \& Reynolds, R.T. (1993). Habitable zones around main sequence stars. Icarus 101, 108-128.

Williams, D.M., Kasting, J.F. \& Wade, R.A. (1997). Habitable moons around extrasolar giant planets. Nature 385, 234-236.

Williams, D.M. \& Pollard, D. (2002). Earth-like worlds on eccentric orbits: excursions beyond the habitable zone. International Journal of Astrobiology, in press.

\section{Circularly polarized reflectance properties of leaves and possible application to remote sensing}

Ramon Wolstencroft $\bullet$ Royal Observatory Edinburgh, George Tranter • Imperial College

Plant leaves are assembled from arrays of chiral molecules of a single hand. A fraction of the sunlight incident on a leaf enters the leaf and is eventually diffusely reflected from the leaf after suffering scattering and absorption within the leaf. Circular dichroism of the chiral molecules, plus the molecular architecture within the leaf, impose a small circular polarization on the emerging light. The light that is specularly reflected from the leaf surface (without entering the leaf) is linearly polarized only. Laboratory measurements made by one of us (rdw) in the 1970 s on one type of leaf showed this effect (at the $1 \%$ level) and we are now carrying out circular spectropolarimetry of a variety of leaves to establish whether this effect could be large enough for a wide variety of leaf species to provide an application for planetary remote sensing. Measurements in the spectral range $185-800 \mathrm{~nm}$ have been made on several plant species and the results will be presented here.

\section{Habitable planets}

\section{Radiative environments of red dwarf flare star habitable planets}

Andrey Andreeshchev • University of Texas, Austin, John Scalo • University of Texas, Austin, David Smith $\bullet$ Harvard; University of Texas, Austin

Low-mass red dwarf stars are the most common stars in the Galaxy. Conventional habitable zone planets orbiting these stars easily avoid atmospheric freeze-out even if tidally locked (Joshi et al. 1997), making them potentially the most common life-bearing systems. They remain active, with strong frequent flares and powerful coronal X-ray emission, for much of their lives, and hence present an interesting radiation environment for the development of life. The physical properties of over 300 observed $\delta \mathrm{Me}$ flare stars are estimated from observations and theoretical models. We compute statistical properties of habitable zone planets for the stellar sample, relying on detailed flare statistics for about 30 of the $\delta \mathrm{Me}$ stars and observed and theoretical spectra. The Xray fluxes above the atmosphere are enormous, but the fraction of energy that affects atmospheric chemistry or surface biology involves radiative transfer considerations. We treat these with Monte Carlo transport simulations. We also find that, including the flare luminosity, the time-averaged UV flux in the habitable zone is comparable to the terrestrial value for many of the $\delta \mathrm{Me}$ stars. Thus, the radiation environment is not highly UV deficient as commonly supposed, but is highly intermittent. The flare irradiation rate as a function of flux and fluence in such an environment is calculated.

\section{The size distribution of planetesimals interior to I AU}

Rory Barnes $\bullet$ University of Washington, Thomas Quinn $\bullet$ University of Washington, Jack J. Lissauer $\bullet$ NASA Ames Research Center, Derek C. Richardson $\bullet$ University of Maryland

We present simulations that examine collisional accretion amongst planetesimals in the terrestrial zone around a $1 \mathrm{M}$ star. The planetesimals begin as $1 \mathrm{~km}$ bodies with a density of $3 \mathrm{~g} / \mathrm{cc}$. Specifically we examine small ( $\sim 10-3$ AU square) patches of $\sim 105$ particles inside $1 \mathrm{AU}$. The simulations presented here are the highest resolution representation of this phenomenon, and are therefore the best estimate to date of the initial planetesimal size distribution.

\section{Terrestrial planet formation in extrasolar systems John Chambers $\bullet$ NASA Ames Research Center}

To date, more than 70 planets have been discovered orbiting main sequence stars using the Doppler radial velocity technique. These planets have masses comparable to Jupiter and are thought to be gas giants composed mostly of hydrogen and helium. Currently, rocky terrestrial planets like Earth cannot be detected using the Doppler technique, so the existence and abundance of extrasolar Earth analogues is unconstrained. However, the presence of giant planets orbiting a star plays a substantial role in determining the existence and characteristics of terrestrial planets in the same system. For example, Jupiter and Saturn almost certainly prevented the formation and survival of terrestrial 
planets in the asteroid belt, while they allowed rocky planets to survive closer to the Sun. Here I examine the effect of different giant-planet configurations on the type of terrestrial planets that are likely to form. I will present the results of several tens of $N$-body integrations of the late stages of terrestrial planet formation in the presence of giant planets. These simulations show that the masses and orbits of giant planets strongly constrain the regions in which Earth-like planets can form, and in some cases prevent the formation of any planets in the star's habitable zone. Simulations such as this can be used in conjunction with existing programmes for giant-planet detection when choosing candidate stars for planned missions to detect Earth-sized planets.

\section{Laboratory studies of butane nucleation on Titan haze analog and thin organic films: application to Titan's clouds \\ Daniel B. Curtis $\bullet$ University of Colorado, Boulder, David L. Glandorf \\ - University of Colorado, Boulder, O. Brian Toon • University of Colorado, Boulder, Margaret A. Tolbert $\bullet$ University of Colorado, Boulder, Christopher P. McKay $\bullet$ NASA Ames Research Center, Bishun N. Khare $\bullet$ NASA Ames Research Center}

Titan, Saturn's largest satellite, has a thick nitrogen/methane atmosphere. In Titan's upper atmosphere, the nitrogen and methane are struck by incoming solar radiation and form a photochemical haze of solid particles, dubbed tholins, which fall through the atmosphere. In Titan's lower atmosphere, methane is saturated or supersaturated with respect to nucleation and may form clouds. If these clouds form, it is likely that the haze particles act as condensation nuclei. The properties of the clouds will affect Titan's radiation budget and climate. To better characterize the properties of Titan's methane clouds, we have measured the saturation ratio required to obtain butane nucleation, $S_{\text {crit }}$, on Titan tholin material and thin organic films of hexane and acetonitrile. We find a critical saturation ratio for butane nucleation on tholin particles of $S_{\text {crit }}=1.30 \pm 0.06$, suggesting that high supersaturations are required for nucleation. If methane is similar to butane, we expect high supersaturations of methane in Titan's atmosphere. This could favor the formation of a small number of large particles, consistent with recent measurements of methane rain or snow on Titan.

\section{The runaway greenhouse effect on Earth and its implication for other planets}

Maura Rabbette $\bullet$ NASA Ames Research Center, Christopher McKay - NASA Ames Research Center, Peter Pilewskie $\bullet$ NASA Ames

Research Center, Richard Young $\bullet$ NASA Ames Research Center, Sam Clanton $\bullet N A S A$ Ames Research Center

The primary greenhouse gas in the Earth's atmosphere is water vapor. It is an efficient absorber of outgoing long-wave infrared radiation. When surface temperature increases, increased evaporation of water from the surface further increases water vapor in the atmosphere, causing more of an atmospheric greenhouse effect. This in turn further increases the surface temperature. If this cycle continues unchecked a runaway greenhouse occurs. Venus is thought to be an example where the runaway greenhouse caused the loss of essentially all water from the planet. The runaway greenhouse is also a factor in determining the habitable zone of extrasolar planets. Planet habitability is a principal topic in astrobiology, a field for which Ames has major responsibilities. There is observational evidence that the runaway greenhouse effect may occur locally on Earth. Using Earth science satellite data and other in situ data we propose to model the signature of the runaway greenhouse. This will allow us to determine whether in fact the runaway greenhouse does occur locally on Earth. If so, we will gain key insights into the Earth's climatology, as well as the processes involved in determining planet habitability.

\section{Venus: a forgotten frontier for astrobiology}

Dirk Schulze-Makuch • University of Texas, El Paso, Louis Irwin • University of Texas, El Paso, Bartlomiej Rzonca $\bullet$ University of Texas, El Paso

With their similar size, chemical composition, and distance from the Sun, Venus and Earth may have shared a similar early history. Surface conditions on Venus are now too extreme for life as known on Earth, but early in the solar system when the Sun was fainter, Venus probably had abundant water and favorable conditions for the origin of life. Given the pervasiveness and persistence of life under stabilizing selection in static environments, it is possible that life could still be present in restricted environmental niches. If Venus once teamed with life, there would be two principal environmental niches to which life could have retreated. First, microbes could have adapted to life in the atmosphere. Since the high pressure on Venus keeps the atmosphere in a thick, liquid-like state, a suitably adapted organism could float in a lifesustaining layer of the atmosphere, migrating as needed by changing density to harvest energy from light, pressure, moisture, or chemical gradients. Secondly, life could have persisted beneath the surface of Venus. Since heat flow from the interior to the surface of Venus is estimated to be relatively low at about $0.06 \mathrm{~W} / \mathrm{m}^{2}$, subsurface temperatures may fall low enough for water to be in a liquid state, where life could thrive on chemotrophic sources of energy. While the logistics of robotic exploration for life on Venus would be challenging, the relative ease of reaching Venus, the plausibility that life could have arisen there, and the possibility that remnants of that life could persist in selected environmental niches argue against discounting Venus as a target for astrobiological investigation.

\section{Oxidation of organic carbon by high pressure hydrothermal processing}

Henry Scott • Geophysical Laboratory, Russell Hemley $\bullet$ Geophysical Laboratory, Rick Ryerson $\bullet$ Lawrence Livermore National Laboratory, Quentin Williams • University of California at Santa Cruz

The three known requirements for life on Earth are liquid water, organic carbon, and energy; it is well known that there are few locations in our solar system where these conditions are extant. After Earth, the candidate bodies are large icy satellites: Jupiter's Ganymede and Europa and potentially Neptune's Triton and Saturn's Titan. The evidence for liquid water in Europa and Ganymede is strong, based on surface observations and magnetic data; indeed, a liquid metallic core is inferred from Ganymede's magnetic data, thus implying interior temperatures $>1000^{\circ} \mathrm{C}$. Furthermore, organic carbon has been observed on the surfaces of Europa and Ganymede. Potentially, high-temperature rockwater interactions occur at the base of Ganymede's thick $\mathrm{H}_{2} \mathrm{O}$ layer (pressure $=\sim 1.5 \mathrm{GPa}$ ), which are similar to hydrothermal vents on Earth where terrestrial life may have originated, but at much higher pressure. The stability of organic carbon under the high-pressure hydrothermal conditions of Ganymede is not well known. Simple thermodynamic calculations show that the anticipated boundary between reduced and oxidized carbon is close to the pressure, temperature, and oxygen fugacity conditions expected deep within Ganymede. We have conducted high-pressure, high-temperature experiments and calculations to explore the stability of organic carbon at conditions relevant to Ganymede's deep interior.

\section{Constraints in the coupling star-life}

Luigi Sertorio $\bullet$ University of Torino, Giovanna Tinetti $\bullet$ Jet Propulsion Laboratory; University of Southern California

If life is sustained by a process of photosynthesis, not necessarily the same existing on Earth, the surface temperature of the star and the orbit of the host planet cannot be whatsoever. In fact the global life cycle, no matter how complicated, must contain in general an upper photochemical branch and a lower dark branch, characterized by a higher and a lower temperature. These two temperatures are star-orbit related. The velocity along the cycle or, in other words, the power of the life machine, depends in general on several other parameters. First of all the Gibbs photon availability, which is a star-orbit parameter and is the input for the upper branch. Then follows the energy cascade that develops along the organic web with a large number of interactions and typical times that must match the typical times generated by the combination of spin value and orientation, eccentricity and precession. Finally, the capacity of the web to keep the global life cycle running along the life span of the star, comes from some inner form of self-endurance and self-balance. 
The property of not being transient could be the right way of introducing the concept of intelligent life.

\section{Possible exobiological sites on Titan}

Michael Simakov $\bullet$ Group of Exobiology, Institute of Cytology

The Saturnian moon Titan is one of the Solar system bodies that are very interesting from an exobiological point of view. Its nitrogen-rich atmosphere like that of Earth and possible internal ocean provide some new exobiological niches. The most appropriate sites for biological and/or prebiological activity at present are: (1) the upper layer of the internal water ocean when a temperature and pressure are suitable for living processes; (2) water pockets and liquid veins inside the icy layer; (3) places of cryogenic volcanism; (4) macro-, mini- and microcaves in the icy layer connected with cryovolcanic processes; (5) the brine-filled cracks in the icy crust caused by tidal forces; (6) liquid water pools on the surface originating from meteor strikes; (7) sites of hydrothermal activity on the bottom of ocean.

The most recent models of Titan's interior lead to the conclusion that a substantial liquid layer exists today under a relatively thin ice cover inside Titan. Such a putative ocean probably contains an array of endogenic materials incorporated into the satellite during its formation and released during planetary differentiation, including considerable mineral and organic compounds, which could help support life and prebiological processes there.

We can see that all the conditions needed for exobiology - liquid water which exists over a long geological period, complex organic and inorganic chemistry and energy sources for support of biological processes exist on this Saturnian moon. On Earth life exists in all niches where water exists in liquid form for at least a portion of the year. Subglacial life may be widespread among such planetary bodies as Jovanian and Saturnian satellites.

\section{Chemical and astrobiological effects of ionizing irradiation on planetary atmospheres}

David Smith • Harvard University, John Scalo $\bullet$ University of Texas, Austin, J. Craig Wheeler $\bullet$ University of Texas, Austin

Monte Carlo simulations of gamma-ray and hard X-ray irradiation of planetary atmospheres are presented, with an emphasis on astrobiological implications involving atmospheric chemistry and direct surface mutational and sterilization effects. Possible radiation sources include flares from late-type parent stars, gamma-ray bursts, and gamma-ray lines from supernovae. We present transmitted spectra as a function of altitude, incident spectrum, angle of incidence, and atmospheric column density. Independent of atmospheric composition, the fluence and spectrum at the ground are partly controlled by Compton downscattering high in the atmosphere to energies $\sim 50 \mathrm{keV}$, below which the atmosphere becomes opaque due to strong photoelectric absorption. The surface fluence of ionizing radiation is found to depend roughly exponentially on the column density. Our calculations suggest that organisms at the Martian surface would not be protected from ionizing radiation by the existing atmosphere. In addition, secondary electrons from these processes are capable of exciting UV spectral lines whose yield can be a significant fraction of the incident ionizing radiation. Depending on the strength of various UV atmospheric shielding components, a biologically significant dose of soft UV radiation can reach the ground even for atmospheres that are very optically thick to ionizing radiation.

\section{Target selection for SETI: a catalog of habitable systems}

Margaret Turnbull $\bullet$ University of Arizona, Jill Tarter $\bullet$ SETI Institute We have created a catalog of potentially habitable stellar systems for use as an expanded target list for the Search for Extraterrestrial Intelligence with the Allen Telescope Array. Here we: (1) define our criteria for a "habitable" stellar system and (2) investigate which stars in our neighborhood may be habitable to Earth-like life. We have based this assessment on the currently available information on variability, multiplicity, spectral type, calcium $\mathrm{H}-$ and $\mathrm{K}$-emission, X-ray emission, rotation, metallicity and kinematics for each star having high-precision parallax data in the Hipparcos Catalog. Starting with a database of 118218 stars, we have systematically eliminated "undesirable" objects to leave a list of $\sim 19000$ Hipparcos stars in the Catalog of Habitable Systems.

\section{Life beyond Earth}

\section{SETI and the postbiological Universe \\ Steven Dick $\bullet$ U.S. Naval Observatory}

The 12-15 billion-year timescale of cosmic evolution, and the necessity of viewing cultural evolution as an integral part of cosmic evolution and the Drake equation, argue that we may live in a postbiological universe, one in which the majority of intelligence has evolved beyond flesh and blood intelligence to artificial intelligence (AI). On Earth cultural evolution vastly outstrips biological evolution, leading some artificial intelligence pioneers (Moravec 1988; Kurzweil 1999) to predict that AI will replace humans in a few generations. $L$, the lifetime of a technological civilization, need not be very long for biological intelligence to be replaced. We propose the Intelligence Principle of cultural evolution, which states that the maintenance, improvement and perpetuation of intelligence is the central driving force of cultural evolution, and that to the extent that intelligence can be improved, it will be improved. Under this principle, over the 6 billion years that intelligence may have evolved in the universe, postbiologicals would vastly outnumber biologicals. The real possibility - perhaps amounting to probability - of a postbiological universe calls for a sweeping reconsideration of SETI assumptions and strategies.

\section{Space-based optical SETI}

\section{Steven Kilston $\bullet$ Ball Aerospace \& Technologies Corporation}

Several new space observatories now planned for astronomical observations, both for astrophysics and for detection and characterization of extrasolar planetary systems, include Kepler, the Next-Generation
Space Telescope (NGST), and Terrestrial Planet Finder (TPF). These and others seem to offer serendipitous potential for SETI at optical wavelengths, due to the following variety of features: (1) calibrated, continuous observations of a particular planetary system or a wideangle region containing many possible systems; (2) wavelength coverage unattainable through the Earth's atmosphere; (3) high photometric accuracy to detect small signal changes; and (4) blocking (nulling) most of a star's light, to detect the very faint signals from objects close to the star. We suggest how these new space observatories might be used for optical SETI, and provide estimates of their potential performance in terms of the technical capabilites of the possible transmitting civilizations.

\section{Basic conditions for transition of biological into intelligent life \\ Vladimir Kompanichenko $\bullet$ Institute for Complex Analysis}

The main planetary and geological conditions, which provide successful advancement of life from the simplest to the intelligent level, have been considered. Once having appeared, living beings display an inevitable tendency to evolve. Under the following favorable conditions an intelligent civilization has a good chance to develop from primary forms of life on a planet: (1) very long period of favorable temperature conditions, which are necessary for laying the foundation of life on a planet, depending mainly on planetary orbital characteristics; (2) a comparable ratio of ocean and land on a planet (optimal thickness of the hydrosphere) that makes possible initial evolution of life in the 
ocean and its further evolution on land; (3) sufficient starlight and optimal cloud cover (rains) that provide growth of plants, transforming light energy of the star into biochemical energy of the biosphere.

SETI@home science processing: a status report Eric Korpela $\bullet$ University of California, Berkeley, Steve Fulton • University of California, Berkeley, Matt Lebofsky • University of California, Berkeley, Jeff Cobb • University of California, Berkeley, David Anderson $\bullet$ United Devices, Dan Werthimer $\bullet$ University of California, Berkeley

The SETI@home project is a distributed computing venture that has attracted over 3.5 million volunteers. These volunteers have collectively analyzed over two years of observations at Arecibo in a search for extraterrestrial intelligence. They have donated over $1.3 \times 10^{21}$ floating point operations to this effort. Combined they have detected nearly a billion unique potential signals. The vast majority are due to random noise fluctuations. A minority are due to radio frequency interference. None have yet been determined to be extraterrestrial in origin.

We will briefly describe the processing performed by SETI@home and the distribution of the hits in power, pulse period, frequency, and sky coordinates. We will describe the steps taken to determine which candidates are most likely to be extraterrestrial in origin, and present examples of the best candidates.

\section{Planetary materials and astroecology: planetary microcosm tests of Martian and carbonaceous chondrite meteorites/asteroids: an assessment of bioresources in the solar system \\ Michael N. Mautner • Lincoln University, Lincoln, New Zealand} Small planetary microcosms, based on Martian and carbonaceous chondrite meteorites, were used to assay meteorites and analogs. The Murchison CM2 meteorite contains high levels of extractable $\mathrm{Ca}, \mathrm{Mg}$, $\mathrm{K}_{\text {and }} \mathrm{SO}_{4}^{-} \mathrm{S}$, while the Martian meteorites DaG 476 and EETA 79001 contain high levels of the essential nutrients nitrate and phosphate. The nutrients are extractable over a wide range of planetary conditions, including early $\mathrm{CO}_{2}$ atmospheres. Biological responses were tested by mesophilic algae and Asparagus officinalis tissue cultures, which proved useful discriminating bioassay agents. The assays ranked the biological fertilities as Martian basalts $>$ terrestrial basalt, agricultural soil $>$ carbonaceous chondrites, lava ash $>$ cumulate igneous rock. Fertility ratings by planetary microcosms are useful in experimental astroecology, for targeting searches for past life. In relation to the future, the results suggest that asteroids and Martian basalts can provide fertile soils for planetary terraforming and space colonization. The nutrient contents of carbonaceous asteroids can sustain a biomass larger by a factor of $10^{3}$ than currently on Earth. The $10^{18} \mathrm{~kg}$ sustainable biomass will allow substantial biological and human expansion in the Solar System.

References

Mautner, M.N. (2002). Planetary resources and astroecology, Astrobiology

$\mathbf{2}$, in press; E-file at http://www.astroecology.com

\section{Quantum mechanical search for molecular quantum computing living systems}

Vykintas Tamulis $\bullet$ Institute of Theoretical Physics and Astronomy, Jelena Tamuliene • Institute of Theoretical Physics and Astronomy Quantum mechanically $a b$ initio and density functional theory-timedependent designed light-driven, single supermolecular devices based on fullerene, biliverdin and photoactive molecules and supermolecules could form the basis for logically controlled organic molecular machines and molecular classical and quantum computers. ${ }^{1}$ The design of basic elements of quantum computers is based on proton NMR calculations on biliverdin derivatives that generate up to ten quantum bits (QuBits) and EPR estimations in endohedral fullerenes possessing up to six QuBits for quantum computation.

These organic and organo-metallic molecular elements of quantum computers could be the origin of quantum computing molecular living systems at the low temperatures found in planets, comets, interstellar dust, etc. Let us imagine that a molecular quantum computing device with ten QuBits is able to move and self-organize to allow supramolecular assembly by using attached light driven azo-dye motors. This supramolecular assembly might allow long-time quantum coherence between the parts of $10 \mathrm{QuBits}$ generating biliverdine derivatives at low temperatures in space. The assemblies possessing long-time coherence states could accept quantum information from the environment and perform quantum computation that further lead to self-organization into more complicated quantum computation living systems and to form a quantum computation brain.

Such a molecular quantum computing cloud may absorb magnetic and light energy from planets and stars, perform information computation and move in space by using the light wind, collecting all the necessary molecules for growth and multiplication. We would like to advise the inclusion of experimental equipment to search for quantum computing living systems in the expeditions to Mars and other Solar system planets. Possibly this molecular quantum computing kind of living system may exist in the higher levels of the Earth's atmosphere. References

1 Tamulis, A., Rinkevicius, Z. \& Tamuliene, J. (2001). Molecular classical computer basic elements. In Decoherence and Implication in Quantum Computation and Information Transfer, NATO Science Series III Computer and Systems Sciences vol. 182, eds Gonis, T. \& Turchi, P.E.A., pp. $358-365$,

\section{SETI and the 12 meter telescope}

Peter Vekinis - Steward Observatory/University of Arizona,

Tom Wilson $\bullet$ Steward Observatory/University of Arizona

Dr Tom Wilson and Peter Vekinis, both of the University of Arizona, Tucson, discuss new avenues for SETI Research at millimeter wavelengths. One of these avenues is positronium emission at around $200 \mathrm{GHz}$, another is based on natural frequencies of extremely powerful cosmic emissions, such as those in the Orion nebula, still further is SETI emissions synchronized to a natural event and the fact that advanced ETIs will use advanced frequencies, such as millimeter waves and above. One clearly assumes that advanced civilizations will be totally communications oriented, with no knowledge of frequencies currently used on Earth and no way of being able to transmit or receive them. In addition, the Steward Observatory, University of Arizona operated 12 meter telescope will be presented as a tool to be used for SETI observations. This will include descriptions of the receivers, back-ends and SETI-specific attachments.

\section{Searching for ET with help from three million volunteers:SETI@home, SERENDIP, and optical SETI at UC Berkeley}

Dan Werthimer $\bullet$ University of California, Berkeley, David Anderson $\bullet$ University of California, Berkeley, Jeff Cobb $\bullet$ University of California, Berkeley, Paul Demorest $\bullet$ University of California, Berkeley, Eric Korpela $\bullet$ University of California, Berkeley, Geoff Marcy • University of California, Berkeley

We present results from two radio and two optical SETI programs at the University of California, Berkeley. The SERENDIP IV sky survey searches for radio signals at the 300 meter Arecibo Observatory. SERENDIP uses a 168 million channel spectrum analyzer and a dedicated receiver to take data 24 hours a day, year round. The sky survey covers a $100 \mathrm{MHz}$ band centered at the $21 \mathrm{~cm}$ line $(1420 \mathrm{MHz})$ and declinations from -2 to +38 degrees. SETI@ home uses desktop computers of 3.5 million volunteers to analyze 50 Terabytes of data taken at Arecibo. The SETI@ home sky survey is 10 times more sensitive and searches a much wider variety of signal types than SERENDIP IV but covers only a $2.5 \mathrm{MHz}$ band. SETI@ home is our the planet's largest supercomputer, averaging 25 Tflops. SETI@home participants have contributed 900000 years of computing time so far. Berkeley's optical pulse search looks for $\mathrm{nS}$ time scale pulses at optical wavelengths. It utilizes an automated 30 inch telescope, three ultra-fast photomultiplier tubes and a coincidence detector. The target list includes F, G, K and M stars, globular cluster and galaxies. A second optical SETI program searches for narrow-band continuous signals using spectra taken by Marcy and his colleagues in their planet search at Keck observatory. 


\section{Humans in an astrobiological context}

\section{Chronoastrobiology: mechanisms of infradian endogenous biospheric cycles on Earth, near- matching physical environmental cycles \\ Franz Halberg $\bullet$ University of Minnesota, Minneapolis, Germaine Cornélissen $\bullet$ University of Minnesota, Minneapolis, Robert B. Sothern - University of Minnesota, Minneapolis, Yoshihiko Watanabe $\bullet$ Tokyo Women's Medical College, Tokyo, Japan, Piero Faraone $\bullet$ CIFA, Brussels, Belgium (located in Milan, Italy), Kuniaki Otsuka $\bullet$ Tokyo Women's Medical College, Tokyo, Japan \\ We document the critical mass of chronoastrobiology by biospheric} cycles of (a) $\sim 7$ (circaseptan) and $\sim 27$ days and $\sim$ half a year, and (b) $\sim 10.5,-21$ and -54 yrs, near-matching non-photic cycles in solar, galactic (a) or geomagnetic (b) activity in demography and 17ketosteroid excretion self-studied for $15 \mathrm{yrs}, 11$ psychophysiologic variables ( $\sim 5$ times a day, 34 yrs), blood pressure and heart rate at halfhour intervals (14 yrs), analyzed by rhythmometry, cross-spectra for coherence, subtraction-addition procedures where the physical environment is removed and replaced by its cycles. In anthropometric variables, e.g. neonatal weight and length, the amplitude of a circadidecadal cycle was larger than the circannual amplitude. The circaseptan amplitude in heart rate during the first weeks of human life is larger than the circadian amplitude in 164 neonates. For heart rate, inheritance of the circadian amplitude and the circaseptan period is suggested by studies on twins. Circadecadals modulate circaseptan sectoring (mutations?) in air bacteria and unicellular oxygen production. Non-photic resonances in many demographic variables including motivation and crime are genetically anchored, as is testable by non-overlapping $95 \%$ confidence intervals, cross-spectral coherence and/or subtraction and addition; chronomics are anchored in genomics ${ }^{1}$.

References

${ }^{1}$ J. Geront. A 2001 56, M304-24.

Life, systems, theory

Eric Kratzer • Kingwood College

Evolution drives the construction of new cellular mechanisms, enabling organisms to seek out and populate environmental niches. Molecular mechanisms of information perpetuation, adaptation, and response to environment are illustrated in the context of evolution. Information processing modes (in parallel) or levels (in hierarchy) of signal transduction are the molecular basis of life, via forced specialization and compartmentalization.

Evolution itself demands the creation of more complex forms of information transfer and signal transduction, to what end?

The transfer and transduction of information drives the activity of a living system, through the creation of more convoluted information pathways, thus increasing the relative disorder of the larger system through increasing the levels of, layers of, and compartmentalization of biological information transfer.

This putative evolutionary mechanism of entropic increase (life) supports the view that non-individual behavior, intelligence, and perhaps even consciousness, were destined to arise from biological systems.

\section{Outreach/education}

\begin{abstract}
A general distributed computing platform for SETI David P. Anderson $\bullet$ Space Sciences Laboratory, U.C. Berkeley, Eric Korpela $\bullet$ Space Sciences Laboratory, U.C. Berkeley, Jeff Cobb $\bullet$ Space Sciences Laboratory, U.C. Berkeley, Bob Bancay • Space Sciences Laboratory, U.C. Berkeley

SETI@home pioneered the use of global distributed computing for radio SETI. As we prepare to expand the project, we are redesigning the "platform" - the software system that distributes data, manages computation, and collects results. The new platform will have many improvements. It will be cleanly separated from the application, allowing several applications (e.g. analysis algorithms for single- and multi-beam receivers) to run simultaneously, and allowing applications to be updated without requiring users to download and install software. In addition, the platform will eliminate network bandwidth bottlenecks by allowing data to be sent from multiple servers. This will allow us to perform SETI searches over wider frequency bands.
\end{abstract}

\section{ExoQuest: astrobiology in high school}

John Baro $\bullet$ Center for Educational Technologies, Steven Croft • Center for Educational Technologies

Are we alone in the universe? This is a question of immense popular interest and a scientific issue of profound importance. ExoQuest, a multimedia educational CD developed at the NASA Classroom of the Future, uses this question as a framework for integrating current research in astrobiology into the grade 7-9 curriculum. Within the context of astrobiology, students explore topics including astronomy, evolution, paleontology, biology, earth science, physics, chemistry, geology, and remote sensing.

With ExoQuest, students travel on virtual journeys to destinations in the solar system and beyond. Their trips are based on past, present, and future NASA missions. At each destination students conduct investigations that include hands-on and simulated experiments. Each of 12 investigations poses problems that focus on different areas of research, providing an interdisciplinary approach to science and the scientific method.

In addition to the activity modules, the "Experts in Science" $\mathrm{CD}$ included with ExoQuest creates links between students and scientists at NASA and other research organizations. In video interviews with 27 world-class experts in astrobiology, students are introduced to firsthand information about scientists' personal lives, their daily activities as scientists, their unique views on their fields of study, and their career paths.

\footnotetext{
The evolution of a novel astrobiology course

Arthur Bowman $\bullet$ Hampton University, Wayne Pryor $\bullet$ Hampton

University, Brendlyn Faison • U.S. Department of Energy, Scott Bailey - University of Alaska, Nakia Parham

With National Aeronautics and Space Administration support (NGT 252261), the Departments of Biological Science and Physics (Center for Atmospheric Sciences) have developed and implemented a new undergraduate course in astrobiolgy. This course, along with an existing undergraduate course in astronomy, represents this university's initial efforts in the area of space science. The course was collaboratively created by two life scientists (microbiology and genetics/cell biology), one professor of atmospheric science, and one astronomer. The course is designed for students with varying science and mathematics backgrounds, however, students advanced in science and mathematics can be challenged. Instruction includes lectures and discussions, as well as self-paced elements such as reviews of space scientists' biographies and space science books (The Birth of Time by J. Gribbins), critiques of popular movies (Mission to Mars), creation of exhibits, seminars, fieldtrips, stargazing, labs, presentations for general audiences, and research of an astrobiology topic of their choosing. Activities are reinforced by use of video tapes, slides, software, and exhibits. The goal
} 
of this course is to enhance the students' awareness and knowledge of astrobiology, while developing an understanding of the relationship of space sciences to life on Earth and the consideration of life in the universe.

\section{Astrobiology: the search for life - a high school integrated science course \\ Jeff Lockwood $\bullet$ TERC, Jodi Asbell-Clarke $\bullet$ TERC}

Is there life elsewhere in the universe? How did life originate on Earth? What is life's future on Earth and beyond? These are some of the profound and exciting questions we use to stimulate students' interest in science. TERC has developed a middle and high school curriculum that galvanizes student participation and learning with intriguing questions and illuminating activities. Astrobiology, by its very nature, kindles interest and curiosity in students and offers a genuinely exciting entrée to high school science.

Astrobiology is the science of today. The story is being written right before our eyes as the exploration of the universe and the search for life unfolds. In a real sense, astrobiology students become participants in a voyage of discovery, as they engage in a field of study that may soon yield some of the most important scientific discoveries of all time.

Astrobiology is a low-cost, high-impact science course designed to reach students having a wide range of abilities. The year-long curriculum is an inquiry-based, integrated program of study. Through a series of hands-on activities, students explore diverse concepts in chemistry, physics, biology, and Earth and space science.

The astrobiology curriculum was conceived, developed, and written by former classroom teachers. Each chapter of the course has been field tested by dozens of teachers and thousands of students. It's About Time will publish the textbook in late 2002. See the astrobiology curriculum web site at http://astrobio.terc.edu for sample activities, and an outline of the course.

\section{Astrobiology and astroethics}

Michael N. Mautner $\bullet$ Lincoln University, Lincoln, New Zealand Applied astrobiology may make the future of life, and of the universe itself, subject to human designs. These designs can be guided by a panbiotic principle: that the human purpose is to safeguard and propagate life in the universe.

Some issues subject to astroethics:

(1) Public debate and astrodemocracy. Because it affects the shared human future, astroethics may be best formulated through direct democracy instruments. The communal decisions require informed debate where scientific facts, extrapolation and speculation are clearly delineated.

(2) Ethical implications of extraterrestrial life.

a. If ET life is found, there is an obligation to protect it from destructive interference, while allowing us to promote our own life-form.

b. In the absence of ET life, it is imperative that we secure and advance life in space.

(3) Planetary quarantine. An ethical balance is needed between quarantine and human exploration and settlement

(4) Directed panspermia. Life may be best promoted by seeding new solar systems. This may soon be feasible, endowing human action with cosmic purpose. Through transforming the universe, astroethics can have cosmic consequences.

References

Mautner, M.N. (1979). J. British Interplanetary Soc. 32, 419 (and 1997, 45, 653), E-reprints: www.panspermia-society.com; "The future and purpose of life: The science and ethics of seeding the galaxy" see www.Legacy-Books.com

\section{Origin and evolution of the UCLA AstroBiology Society}

Laurel Methot $\bullet$ University of California, Los Angeles, Jason Finley • University of California, Los Angeles

Co-sponsored by the NASA Astrobiology Institute (NAI) at UCLA, and the Center for the Study of Evolution and the Origin of Life
(CSEOL), the UCLA AstroBiology Society (ABS) was founded in 1999 to become the first student-run organization devoted to astrobiology. An interdisciplinary group, ABS unifies undergraduates, graduate students, and faculty of all fields through events and discussions centered around the diversity of themes that compose astrobiology science. ABS provides particular benefit to NAI's Education and Public Outreach efforts as a means of increasing awareness of and appreciation for astrobiology, particularly in the college community. A greater goal of the organization involves networking to seed AstroBiology Societies on other college campuses nation-wide. Additionally, ABS is currently doing research for NAI's AstroBiology Pathfinder program, which will help students at all stages to become astrobiologists.

\section{A minority student ambassador program: innovative methods for presenting interactive science programs to urban area underrepresented groups and minorities \\ Penny Morris $\bullet$ University of Houston Downtown, Marilyn Lindstrom • NASA Johnson Space Center, Jaclyn Allen • Lockheed Martin, Carolyn Sumners $\bullet$ Houston Museum of Natural Science, Victor Obot $\bullet$ Texas Southern University, James Wooten $\bullet$ Houston Museum of Natural Science}

The Ambassador program is a long-term effort funded by the NASA Minority University Initiative to train high school and college students to reach out to minority and under represented groups in the Houston urban area. Ambassadors are chosen for good communication skills, grades, and personal recommendations.

NASA scientists and education specialists in conjunction with the Houston Museum of Natural Science train student Ambassadors to share space science in informal settings. Trained Ambassadors present hands-on activities that can be adapted to a wide age group. Activities include exploring the solar system through the needs of fantasy aliens, understanding volcanic structures and their occurrence on Mars, learning about the search for life in extreme environments beyond Earth, and presenting a planetarium show with a portable planetarium.

The Ambassadors have participated in a variety of venues that includes Sun Earth Day at the Houston Museum of Natural Science, NASA Johnson Space Center Open House, the national meeting of the Society of Mexican American Engineers and Scientists, YMCA day camp programs, and a county-wide outreach program directed towards inner city students called the Bright.

\section{Astrobiology education discussion \\ David Morrison • NASA Astrobiology Institute}

As astrobiology becomes a more mature scientific discipline, many universities are introducing courses or degree programs in astrobiology. Of special interest to many of us is the growth in astrobiology as an introductory science course. Many of these courses are being given to non-science majors, and they may prove to be an excellent way to introduce students to science.

I will convene an open discussion of university-level astrobiology education at the AbSciCon on Monday April 8, from 5:30 to 7:00 pm. The primary focus will be on introductory courses rather than advanced degree programs. I am collecting notes and course descriptions that I will make available at the meeting. This is an opportunity to talk about the role of astrobiology in university and college education, about the content of such courses, and about what we might like to see in the way of texts or other supporting materials. I hope that those who have taught such courses can share their experiences and provide advice to others who are only thinking about this possibility. All are welcome, and I particularly encourage those who are involved in education to attend.

\section{Astrobiology professional organization discussion David Morrison $\bullet$ NASA Astrobiology Institute}

There will be an open discussion on Tuesday April 9 from 5:30 till 7:00 pm of the possibility of planning for an astrobiology professional society, a topic that has arisen in numerous discussions since the last AbSciCon. Many of our colleagues feel that it would be beneficial to the 
astrobiology profession to create such an organization. A professional society could help provide substance and enhance communications within this discipline. It might provide fellowship and job listings as a service to its members. An astrobiology society could organize workshops or meetings. Such a society could also sponsor a journal of astrobiology. I doubt very much if anyone wants to start a new journal, but it is possible that a society might want to "adopt" one of the existing astrobiology journals. Thirty years ago the Division for Planetary Sciences of the American Astronomical Society did just that, creating an affiliation with the privately published journal Icarus. I believe that both the DPS/AAS and the publishers of Icarus have been happy with this association.

I am convening an open discussion for those who are interested in possibly forming an astrobiology society. NASA has no intention of sponsoring such a society, but we can provide a forum for discussion and see how much serious interest exists. I welcome representatives of other societies and the publishers of existing astrobiology journals to participate in this session. Please note that this is a discussion meeting, not a decision-making meeting. We are at the start of this process, not in the end game.

\section{Kinesthetic astronomy \\ Cherilynn Morrow $\bullet$ Space Science Institute, Michael Zawaski $\bullet$ Space Science Institute}

We are developing an innovative, experiential approach to learning basic astronomical concepts. We call our approach "kinesthetic astronomy;" it is intended for learners in both formal and informal educational settings. We are developing a sequence of inquiry-based lesson plans that address astronomical phenomena people can readily see in the sky. The lessons are science-rich and fun. In our first lesson, called "Sky Time" students experience a series of simple body movements (e.g. rotating, revolving, tilting, bending, twisting), that gives them insight into the relationship between time and astronomical motions of Earth (rotation about its axis, and orbit around the Sun), and also about how these motions influence what we see in the sky at various times of the day and year. Other lessons are devoted to lunar motion, meteor showers, and the sky motions of the planets. Future lessons will also address pertinent topics in astrobiology and origins. Field testing with grades 6 and up suggests that kinesthetic astronomy techniques allow learners to achieve a good intuitive grasp of concepts that are much more difficult to learn in more conventional ways. These lessons are fully aligned with national science education standards. kinesthetic astronomy is funded with support from NASA's Office of Space Science/IDEAS grant program.

\section{Identifying student misconceptions in astrobiology Erika Offerdahl $\bullet$ University of Arizona, Edward Prather $\bullet$ University of Arizona, Timothy Slater $\bullet$ University of Arizona}

The purpose of this study was to identify and document student beliefs and reasoning difficulties concerning astrobiology-related topics. This was accomplished by surveying over 2000 middle school, high school, and college (science and non-science majors) students. Students were surveyed utilizing open response questions focused on the definition of life and its limitations. Careful, inductive analysis of student responses revealed that the majority of students correctly identify that liquid water is necessary for life and that life forms can exist without sunlight. However, many students incorrectly state that life cannot survive without oxygen. Furthermore, when students are asked to reason about life in extreme environments, they most often cite complex organisms (such as plants, animals and humans) rather than the more ubiquitous microorganisms. Results of this study were used to develop a set of inquiry-based activities.

\section{Astrobiology in the classroom and beyond: educational opportunities and resources at the Marine Biological Laboratory, Woods Hole, MA}

Lorraine Olendzenski $\bullet$ University of Connecticut

As part of the Astrobiology Institute, we offer teacher enhancement workshops focusing on microbial diversity, evolution and astrobiology.
Our workshops combine lectures from research scientists with lab experiences using hands-on activities appropriate for the middle or high school classroom. Topics of workshops include: Living in the Microbial World; Living Sands: Mapping Time and Space with Forams; Life on Earth and Elsewhere?; and Life on the Edge: Extremophiles.

Microscope (www.mbl.edu/microscope), a web-based resource in microbial diversity developed through the Institute provides an extensive database of high-quality digital photomicrographs and information on habitat and phylogeny of prokaryotic and eukaryotic microorganisms that is useful to both researchers and educators.

Additional opportunities at the MBL for advanced students, researchers, and science writers in areas related to astrobiology include the Workshop on Molecular Evolution, Advances in Genome Technology and Bioinformatics, the NASA Planetary Biology Internship, and the Science Journalism Program. All of these programs are ongoing and available to participants by competitive application. Further information is available at www.mbl.edu.

\section{An online astrobiology course for teachers}

Edward Prather $\bullet$ University of Arizona, Timothy Slater $\bullet$ University of Arizona

Effective inquiry-based instruction requires that teachers possess a detailed understanding of the target concepts to be taught. Because astrobiology is a new field of science bringing together many scientific fields of study, it presents a formidable challenge for most K-12 science teachers. The NASA-funded Center for Educational Resources (CERES) Project (http://btc.montana.edu/ceres/) has designed a set of classroom activities and an accompanying Internet course for teaching astrobiology. These activities have been designed to combine on-line data resources from NASA with the student-centered inquiry instructional strategy emphasized in the National Science Education Standards. The activities have been developed and field-tested by precollege science teachers and university faculty. The accompanying asynchronous Internet course is a 15-week, graduate-level course in astrobiology for teachers. The course integrates the NASA Astrobiology Roadmap, the NRC National Science Education Standards, and the astrobiology curriculum supplements available online at http:// btc.montana.edu/ceres/astrobiology. The two main goals of this course are: (1) to provide information on the central concepts related to the field of astrobiology, and (2) to provide experiences with using studentcentered and inquiry-based curriculum materials for teaching astrobiology.

\section{Graduate education in astrobiology: producing the first real astrobiologists}

W.T. Sullivan, III • University of Washington, James T. Staley • University of Washington

Many educational issues have arisen in the emerging field of astrobiology. At the University of Washington (UW) we have focused on graduate education and the question of how best to produce the new generation of astrobiologists. In 1999 we admitted our first students and now have 15 participants with majors across ten departments. There is no "Department of Astrobiology" - students still receive a Ph.D. in their home department's discipline (e.g. oceanography, microbiology, astronomy), but spend about $30 \%$ of their time earning an additional Certificate in Astrobiology. This talk will describe this pioneering program, how it has been shaped and is still being redefined, and lessons learned.

The program aims to produce a student solidly grounded in one discipline, but so exposed to the other disciplines comprising astrobiology that she or he is familiar with the key concepts and "culture" of other relevant disciplines (e.g. allowing the current literature to be read and understood to a large degree), is oriented towards thinking about problems in a more universal scientific way, and is comfortable with, indeed even desirous of, multidisciplinary collaborative projects, especially those at the heart of astrobiology that link the physical and biological sciences.

We achieve this through: (1) specially designed core courses and seminars, (2) an annual hands-on field workshop for both faculty and 
graduate students (topics have included subterranean microbiology, invertebrate marine biology, paleontology, and choosing a Martian landing site), (3) rotations through labs outside one's own department, (4) graduate research stipends that are attached to the student rather than to the faculty member, and (5) multidisciplinary mentoring and advising as the student chooses and researches his or her Ph.D. thesis.

A natural outgrowth of our program has been a graduate-level textbook, Planets and Life: The Emerging Science of Astrobiology (to appear in 2003), edited by Sullivan and John Baross (UW Biological Oceanography), with about one-third of the material from UW authors (including our students) and the remainder by other experts. The style (which we also request of all speakers in our seminars) is that the first two-thirds of each chapter should be understandable by anyone with a bachelor's degree in a field relevant to astrobiology, with the remainder becoming more technical.

http://depts.washington.edu/astrobio

\section{Using astrobiology to bridge "Earth, Ocean, Space" at the California Academy of Sciences, San Francisco} Carol M. Tang • California Academy of Sciences

Astrobiology is a scientific endeavor that encompasses many different disciplines ranging from geology to biology to astronomy. As such, it provides a strong conceptual basis for illustrating the linkages between atmosphere, lithosphere, hydrosphere and biosphere. For museums that are looking for accessible ways to teach scientific principles, astrobiology can be used as a tool to illustrate the rapidly changing nature of science, the importance of posing testable hypotheses, the role of technology in scientific discovery and the feedbacks between biotic and abiotic factors.

The California Academy of Sciences in Golden Gate Park, San Francisco, consists of a natural history museum, aquarium, and planetarium-Earth, Ocean and Space. In addition, the Academy houses a biodiversity and systematics research program and a significant research collection with over 16 million specimens of plants, animals, fossils, and minerals. Astrobiology can provide a bridge between these different components on the public floor as well as intellectually behindthe-scenes. Because the museum is focused mainly on biological concepts, the Planetarium and docent led-school tours have used the concept of astrobiology to link Mineral and Earth and Space Halls to the other exhibits. Current astrobiological research at the Academy on the biology of thermal springs was highlighted on the museum floor and archived on the web under the "Science Now" exhibit. In addition, in extensive planning sessions for new exhibits, astrobiological concepts appear to have struck a chord with the design team and they are being incorporated in both subtle and direct ways into new exhibits with the guidance of education and research staff.

\section{Johnson Space Center astrobiology education: Fingerprints of Life?: a new web site and CD for the classroom developed by a collaborative team of scientists and educators}

Kay W. Tobola $\bullet$ NASA Johnson Space Center, Jaclyn S. Allen • NASA Johnson Space Center, Rebecca Collier $\bullet$ Killeen Independent School District, Karen Stocco $\bullet$ Pasadena Independent School District, Cheryl Pittman $\bullet$ Pearland Independent School District, Marilyn M. Lindstrom $\bullet$ Johnson Space Center

Fingerprints of Life? is a new web site and CD developed by the Johnson Space Center Astrobiology Education team for use by educators, students and the public. The focus is on helping students to understand how scientists search for traces of microbial life in extreme environments and ancient rocks on Earth and beyond. Classroom activities, PowerPoint presentations, and resources are easily accessed. In the Eye Spy lessons, students view SEM images of familiar objects and microbes to learn how scientists conduct research. Other lessons have students describe and classify critters, experiment with extreme environments, and test the limits of chemical tracers. One slideshow introduces microbes. Another highlights JSC research on fingerprints of life- biomarkers in samples from ancient and modern Earth, and extreme environments. The site includes research about the search for life on Mars and the debate over possible life in Martian meteorites.

Scientist-educator teams worked collaboratively to produce the astrobiology materials. Educators worked with scientists to learn how they look for evidence of microbes in samples. Scientists assisted educators with ideas for realistic activities that would reflect the research.

Materials developed by scientist-educator teams provide both resources and lessons to take real science to the classroom.

http://ares.jsc.nasa.gov/Education/websites/astrobiologyeducation/ index.html

\section{Web based astrobiology program activities at Arizona State University}

Leslee Unser $\bullet$ Arizona State University, Rebecca Davis $\bullet$ Arizona

State University, Jack Farmer $\bullet$ Arizona State University

The Arizona State University astrobiology program has a constantly evolving web site that has become a focal point to highlight ASU-based activities in Astrobiology as well as links to many other astrobiology sites across the country and abroad. This site is serving as an important mechanism to recruit students and educate the public about the activities and achievements of the NASA Astrobiology Institute and, specifically, at ASU. In this presentation we will discuss the major elements of our web-based education and outreach activities.

\section{Stars and planets: astronomy and astrobiology activities for the middle-school classroom \\ Mary Urquhart $\bullet$ NASA Ames Research Center; NRC}

A new series science/math lesson plans designed for grades 6-8 encourages middle-school students to explore topics relevant to both astronomy and astrobiology. In a "Scale Model Solar System," students will investigate the sizes and distances of the Sun and planets using a scale factor of 1-10 billion. The same scale factor is used in "Sizes of Stars" to compare the Sun to other classes of main sequence stars, and to compare stellar and solar system distances in "Distances of Stars." A probability activity is used in "Star Birth" to discover how relative numbers of Sun-like stars compare with those of different masses. In "Lifetimes of Stars" students compare the timeline for the history of life on Earth and stellar lifetimes. They then decide what classes of stars they think astronomers should focus on in the search for planets that might have life. The production of planet- and life-forming elements in stars is introduced in "Death of Stars," as is the future fate of our own Sun. In the final lesson students will bring together many of the concepts explored in previous lessons in a discussion of the issues involved in "Planet Hunting," and will learn about the current and planned efforts to find planets beyond the solar system.

"Stars and Planets" is currently undergoing field-testing, and is available at http://lyra.colorado.edu/sbo/mary.

\section{The search for extraterrestrial life: a museum exhibition and education program}

Martin Weiss • New York Hall of Science

The Search for Extraterrestrial Life will be a permanent exhibition at the New York Hall of Science that will engage visitors in applying the fundamental biology of life on Earth to understanding the search for extraterrestrial life. The exhibition and accompanying programs, including a public discovery laboratory, will engage visitors in investigating:

(1) the breadth of conditions that support life on Earth;

(2) how the critical requirements for life on Earth are shaping the search for life on Mars and other planetary objects;

(3) how the search for extraterrestrial life is informed by the histories and environments of these planets;

(4) the strategies and technologies scientists are developing to detect extraterrestrial life. 
We will describe, using the plans for this exhibition, how science museums can interpret and engage visitors of all ages in discovering the realm of extremophiles and how the knowledge of these organisms is informing our concept of the search for extraterrestrial life.

\section{Astrobiology: recruiting the next generation}

Krisstina Wilmoth $\bullet$ NASA Astrobiology Institute

As a relatively new field, astrobiology must be concerned with its own future. Much of the work in astrobiology will not be realized in a single generation, and therefore it is imperative that new researchers join the fold and continue the work. Recruiting and educating students is an essential part of building a future for the discipline. The NASA Astrobiology Institute (NAI) accomplishes this through the direct training offered at NAI Member sites and through an extensive program in Education and Public Outreach.
Students and the general public are extremely interested in the questions of astrobiology. They send email, write letters and post questions to our website clamoring for more information. In response, NAI developed Ask an Astrobiologist, to answer questions submitted via the website and Astrobiology Pathfinder, an online database which allows students to select from a series of grade levels, areas of scientific interest, and regional location to personalize their paths to astrobiology research as a career. Additional aspects of the site will highlight internships, opportunities at science and technology museums and courses at various colleges and universities to provide a comprehensive experience for the user and to assist in the recruitment of the next generation of astrobiology researchers. In this way, education and public outreach has a direct impact on the growing field of astrobiology. These and other projects are available at the NAI website at http:// nai.arc.nasa.gov 